University of Tennessee Health Science Center

UTHSC Digital Commons

\title{
Understanding the Impact of BCRP and PGP Efflux Transporters on the Disposition of Their Endogenous, Xenobiotic and Dietary Substrates
}

Samit Ganguly

University of Tennessee Health Science Center

Follow this and additional works at: https://dc.uthsc.edu/dissertations

Part of the Pharmacy and Pharmaceutical Sciences Commons

\section{Recommended Citation}

Ganguly, Samit (http://orcid.org/0000-0002-3037-1959), "Understanding the Impact of BCRP and PGP Efflux Transporters on the Disposition of Their Endogenous, Xenobiotic and Dietary Substrates" (2018). Theses and Dissertations (ETD). Paper 481. http://dx.doi.org/10.21007/etd.cghs.2018.0457. 


\title{
Understanding the Impact of BCRP and PGP Efflux Transporters on the Disposition of Their Endogenous, Xenobiotic and Dietary Substrates
}

\author{
Abstract \\ Breast Cancer Resistant Protein (BCRP) and P-glycoprotein (PGP) are membrane-bound efflux \\ transporters that transport multiple chemical classes of compounds and act as barriers to tissue \\ permeability, thereby regulating tissue exposure of their substrates. The role of these transporters in \\ therapeutic resistance of their xenobiotic substrates due to their expression and efflux function at target \\ organs/tissues, especially in brain and intestine, is well established, which has fueled investigation toward \\ identification of their inhibitors. Multiple clinically used drugs and dietary chemicals have been reported to \\ inhibit these transporters and potentially increase the exposure of concomitantly administered BCRP and/ \\ or PGP substrates, which might be desirable (to increase efficacy of the substrate drug) as well as \\ undesirable (as it can affect the disposition of the substrate in tissues where it might cause toxicity). \\ Therefore, drug-drug and food-drug interactions with BCRP and PGP are a major concern in drug \\ development, as recognized by US Food and Drug Administration (FDA), European Medicines Agency \\ (EMA) and International Transporter Consortium (ITC). \\ BCRP and PGP transporters are also known to transport endogenous substrates and have been \\ associated with important physiological functions. This includes an inflammatory bowel disease-like \\ phenotype associated with a non-functional PGP polymorphism (Ala893), while a loss of function \\ mutation in BCRP (Q141K) is known to be positively associated with gout and negatively associated with \\ Parkinson's syndrome. Although a few endogenous substrates of BCRP and PGP have been identified, a \\ systemic understanding of their physiological function and effect on the endogenous metabolome is still \\ lacking. \\ In the current studies, utilizing untargeted metabolomics, coupled with transcriptomic analysis, we sought \\ to understand the endogenous function of Bcrp and/Pgp transporters in rats, by comparing the plasma \\ and cerebrospinal fluid metabolome of wild-type and Bcrp-Pgp double knockout rats. Our findings \\ revealed several putative novel endogenous/dietary substrates of Bcrp and Pgp transporters and \\ established a novel understanding of the physiological function of these transporters. We also identified \\ the anesthetic/analgesic ketamine as a putative substrate of Bcrp and Pgp, and found that ketamine \\ pharmacokinetics (PK), as well as pharmacodynamics (PD), are affected by these efflux transporters. \\ Importantly, the Bcrp-Pgp inhibitor, elacridar, was found to alter the PD of ketamine, indicating that \\ ketamine might be prone toward drug-drug interactions (DDIs) associated with Bcrp-Pgp inhibitors. \\ Finally, utilizing a Bcrp dietary substrate, Pheophorbide $A(P h A)$, we developed an in vivo assay for the \\ assessment of DDI due to inhibition of Bcrp at enterocytes. Our results demonstrated that PhA can be \\ potentially used to create a reliable, high-throughput and less expensive in vivo assay for determining \\ Bcrp-mediated DDIs.

\section{Document Type} \\ Dissertation \\ Degree Name \\ Doctor of Philosophy (PhD)

\section{Program} \\ Biomedical Sciences
}




\section{Research Advisor}

Erin G. Schuetz, Ph.D.

\section{Keywords}

BCRP Drug-Drug Interaction, Efflux Transporter, Ketamine, Metabolomics, PGP

\section{Subject Categories}

Medicine and Health Sciences | Pharmacy and Pharmaceutical Sciences 


\title{
Understanding the Impact of BCRP and PGP Efflux Transporters on the Disposition of Their Endogenous, Xenobiotic and Dietary Substrates
}

\author{
A Dissertation \\ Presented for \\ The Graduate Studies Council \\ The University of Tennessee \\ Health Science Center
}

\author{
In Partial Fulfillment \\ Of the Requirements for the Degree \\ Doctor of Philosophy \\ From The University of Tennessee
}

By

Samit Ganguly

August 2018 
Chapter 3 (C) 2018 by American Society for Pharmacology and Experimental Therapeutics.

All other material C 2018 by Samit Ganguly.

All rights reserved. 


\section{DEDICATION}

"Making a decision was only the beginning of things. When someone makes a decision, he is really diving into a strong current that will carry him to places he had never dreamed of when he first made the decision."

- Paulo Coelho, The Alchemist

To my mother Swapna Ganguly, my father Sailendranath Ganguly, my wife Dr. Kamalika Mukherjee and Late Dr. Tuhinadri Sen, who always stood by me making me stronger than I ever was. 


\section{ACKNOWLEDGEMENTS}

"I am the sum total of everything that went before me, of all I have been seen done, of everything done-to-me. I am everyone everything whose being-in-the-world affected was affected by mine. I am anything that happens after I'm gone which would not have happened if I had not come." - Salman Rushdie, Midnight's Children

I would like to take this opportunity to acknowledge everyone who has directly or indirectly helped me be where I am today.

I would like to first acknowledge my mentor, Dr. Erin Schuetz, who has helped me think critically, logically and scientifically. While she has given me the freedom to work and learn, her constant guidance and mentoring also gave me directions and focus on my projects. She has always encouraged me to be independent and pursue my own small goals. I will always cherish our long scientific conversations with Dr Schuetz!

I would also like to acknowledge my Ph.D. committee members, Dr. Alex Sparreboom, Dr. Bernd Meibohm, Dr. Taosheng Chen, Dr. Ryan Yates and Dr. Michael Taylor, for their guidance and advice on my project and at different stages of my professional career. I would also like to acknowledge my lab members, Dr. Amarjit Chaudhry, Dr. Kazuto Yasuda, Cynthia Cline, Dr. Holly Johnson, Jeremy Hunt, Rachel Scheib and Kavya Annu, for their immense help and support. A special thanks also to members of the lab of Dr. John Schuetz, especially Dr. Yu Fukuda and Dr. John Lynch for their advice related to my research work. I cherished my time in the lab and hope to be in touch with all of them, and be a part of this big Schuetz lab family.

I would also like to thank Dr. John Schuetz for his guidance and mentoring and insightful lab meeting discussions. Dr. John Schuetz has helped me learn so much about transporters, thinking critically about transporter biology, transporter assays and also mold me into a confident scientific presenter.

I want to convey my special thanks to Dr. Jessica Roberts for her guidance on my career directions as well as mentoring me to learn more about pharmacometrics. A big thank you to Dr. Carl Panetta as well for mentoring me to learn the basic principles of pharmacometrics. I probably cannot name everyone here, but I would also like to thank everyone I met at the numerous scientific meetings for their immensely insightful discussions about my research work.

My time in Memphis would not have been so wonderful if I also did not have the support of a wonderful group of friends. I have made wonderful memories with them and would cherish them throughout my life. I would also like to acknowledge my friends from AstraZeneca, India, Dr. Abhijeet Deshpande, Vijaykamal Ahuja, Dr. Vishwas KG, Dr. Mahesh Kumar KN, Vijender Panduga, and everyone else who have been my biggest 
support system. I would also want to thank Dr. Vrinda Nandi, Dr. Radha Shandil, Dr. Vinayak Hosagrahara for their mentorship during my stay at AstraZeneca.

My acknowledgement list would not be complete without the mention of Late Dr. Tuhinadri Sen, my first pharmacology professor in India. If it was not for TS, as we lovingly called him, and our brief but intense scientific discussions and his constant words of encouragement, I would not have thought of pursuing research in this field. I know He must be watching over us and probably smiling over my realization!

I would like to thank my parents for being so supportive, and let me pursue my dream while staying away from home. No words and acknowledgement are enough to express my gratitude for them. I feel extremely fortunate and proud to have you beside me. Thank you so much! And special thanks go to my sister Mandira, and specially my nephew Ujan Sinha, for taking care of my home and be my support system always.

Last and definitely not the least, my friend, philosopher and guide, my wife Dr. Kamalika Mukherjee. Thank you for being there with me. Thank you for your confidence, support and for always telling me what is the right thing to do, and not letting me compromise. You have always made me a better version of me, always believed in my abilities more than I did and always pushed me towards excellence. I am so glad to be by your side and get your support and confidence. My sincere thanks to you for being there for me.

At the end of this journey to obtain my Ph.D., I have learnt that it is really not the end of the road, but actually the beginning of a new journey. 


\begin{abstract}
Breast Cancer Resistant Protein (BCRP) and P-glycoprotein (PGP) are membrane-bound efflux transporters that transport multiple chemical classes of compounds and act as barriers to tissue permeability, thereby regulating tissue exposure of their substrates. The role of these transporters in therapeutic resistance of their xenobiotic substrates due to their expression and efflux function at target organs/tissues, especially in brain and intestine, is well established, which has fueled investigation toward identification of their inhibitors. Multiple clinically used drugs and dietary chemicals have been reported to inhibit these transporters and potentially increase the exposure of concomitantly administered BCRP and/or PGP substrates, which might be desirable (to increase efficacy of the substrate drug) as well as undesirable (as it can affect the disposition of the substrate in tissues where it might cause toxicity). Therefore, drug-drug and food-drug interactions with BCRP and PGP are a major concern in drug development, as recognized by US Food and Drug Administration (FDA), European Medicines Agency (EMA) and International Transporter Consortium (ITC).
\end{abstract}

BCRP and PGP transporters are also known to transport endogenous substrates and have been associated with important physiological functions. This includes an inflammatory bowel disease-like phenotype associated with a non-functional PGP polymorphism (Ala893), while a loss of function mutation in BCRP (Q141K) is known to be positively associated with gout and negatively associated with Parkinson's syndrome. Although a few endogenous substrates of BCRP and PGP have been identified, a systemic understanding of their physiological function and effect on the endogenous metabolome is still lacking.

In the current studies, utilizing untargeted metabolomics, coupled with transcriptomic analysis, we sought to understand the endogenous function of Bcrp and/Pgp transporters in rats, by comparing the plasma and cerebrospinal fluid metabolome of wild-type and Bcrp-Pgp double knockout rats. Our findings revealed several putative novel endogenous/dietary substrates of Bcrp and Pgp transporters and established a novel understanding of the physiological function of these transporters. We also identified the anesthetic/analgesic ketamine as a putative substrate of Bcrp and Pgp, and found that ketamine pharmacokinetics (PK), as well as pharmacodynamics (PD), are affected by these efflux transporters. Importantly, the Bcrp-Pgp inhibitor, elacridar, was found to alter the PD of ketamine, indicating that ketamine might be prone toward drugdrug interactions (DDIs) associated with Bcrp-Pgp inhibitors. Finally, utilizing a Bcrp dietary substrate, Pheophorbide A (PhA), we developed an in vivo assay for the assessment of DDI due to inhibition of Bcrp at enterocytes. Our results demonstrated that $\mathrm{PhA}$ can be potentially used to create a reliable, high-throughput and less expensive in vivo assay for determining Bcrp-mediated DDIs. 


\section{TABLE OF CONTENTS}

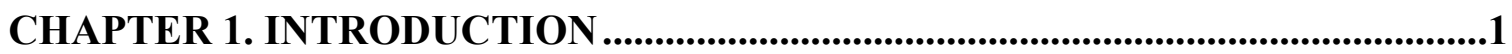

Membrane Drug Transporters..............................................................................

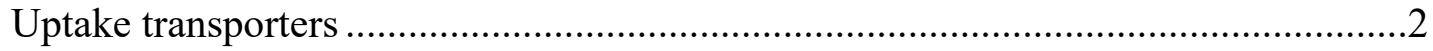

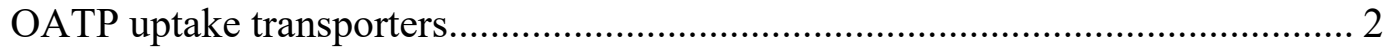

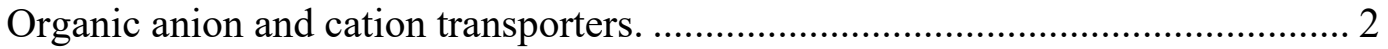

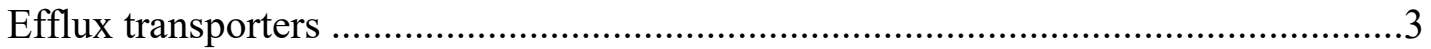

Structure of ABC transporters. ................................................................ 4

Clinical significance of ABC transporters. ................................................. 4

Endogenous substrates of ABC transporters.................................................... 5

Efflux transport and xenobiotic substrates....................................................... 5

Drug-drug interactions and transporter/enzyme substrate overlap ...........................6

Assays for identification of transporter substrates and inhibitors.............................6

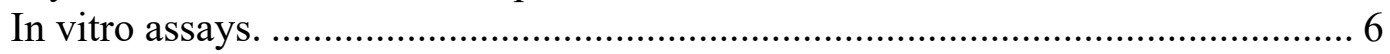

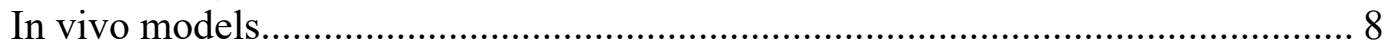

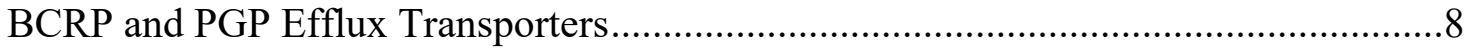

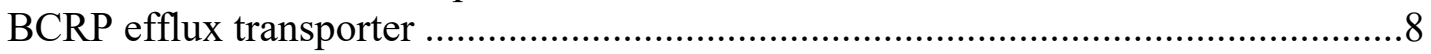

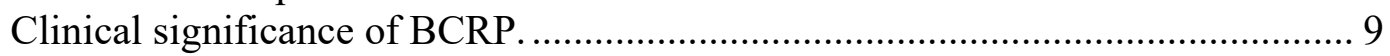

Xenobiotic substrates or inhibitors of BCRP ................................................. 9

Endogenous substrates of BCRP. ................................................................ 9

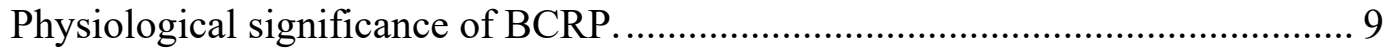

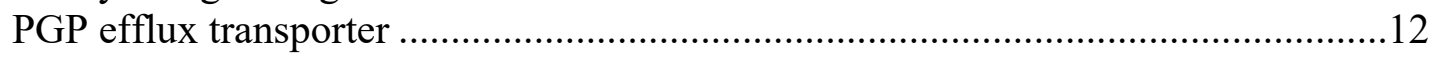

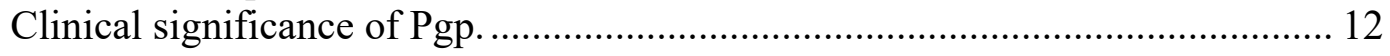

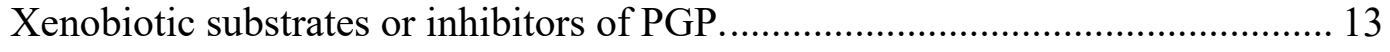

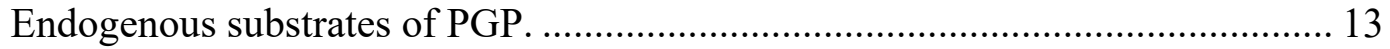

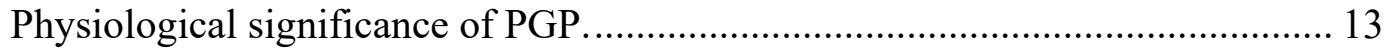

Substrate or inhibitor overlap of BCRP and PGP ...............................................14

Guidance about drug-drug interaction and substrate and/or inhibitor

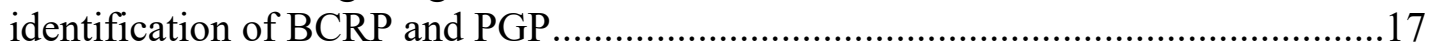

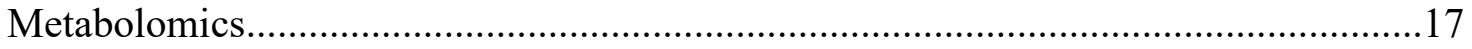

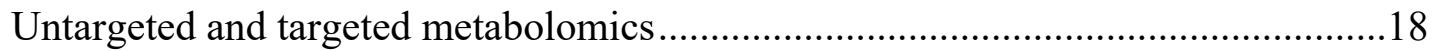

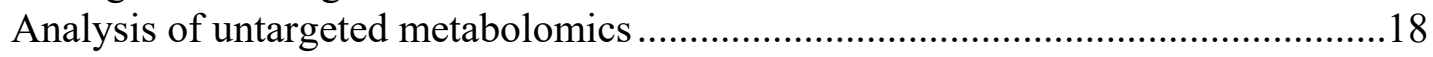

Integration of untargeted metabolomics and transcriptomics ................................19

Use of metabolomics to understand endogenous function of transporters ...............19

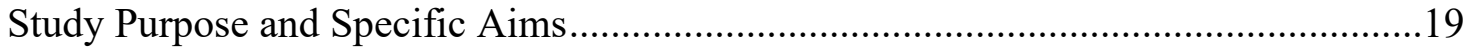

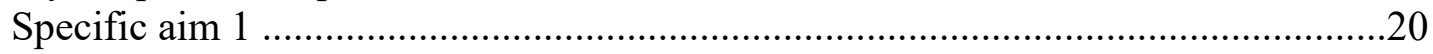

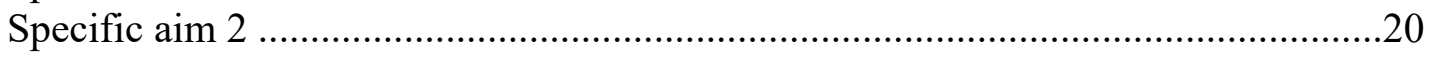

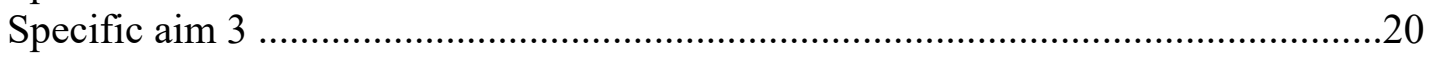

CHAPTER 2. ELUCIDATION OF ENDOGENOUS FUNCTION OF BCRP

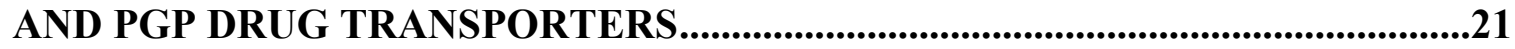

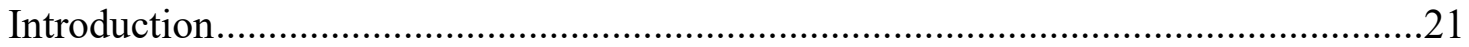

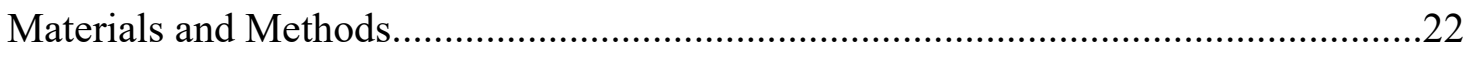

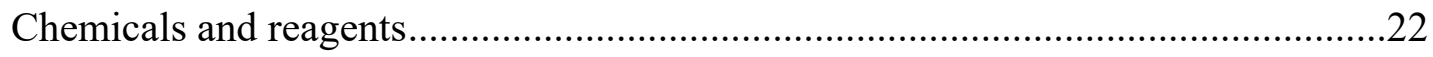


Animal experiments and sample collection ...........................................................23

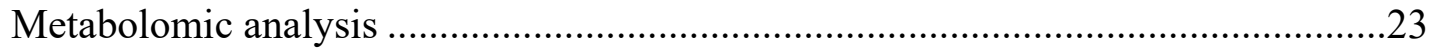

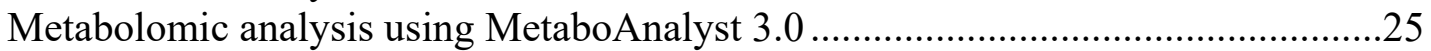

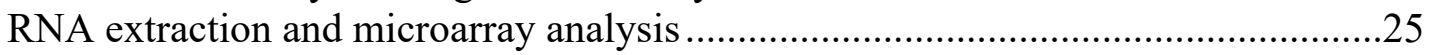

In silico prediction of BCRP and/or PGP putative substrates or inhibitors ...............26

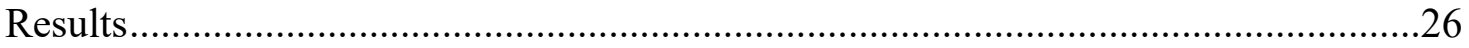

Bcrp and Pgp gene deletion in rats results in altered metabolome of CSF and

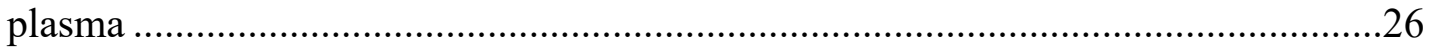

Bcrp and Pgp gene deletion in rats results in altered transcriptome of brain, kidney and liver.

Use of gene expression and known substrate knowledge for understanding metabolomic features

In silico prediction of BCRP and PGP substrate-like properties for the significantly altered metabolites in CSF and plasma of dKO rats .............................58

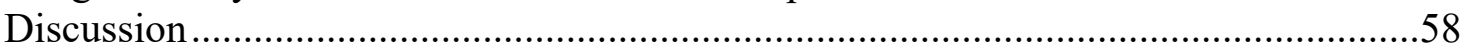

\section{CHAPTER 3. KETAMINE PHARMACOKINETICS AND PHARMACODYNAMICS ARE ALTERED BY PGP AND BCRP EFFLUX TRANSPORTERS IN MICE.....................................................................................66}

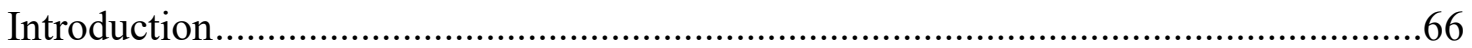

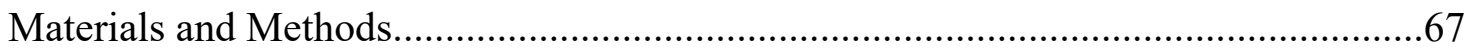

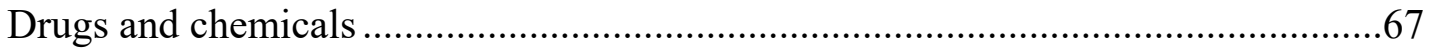

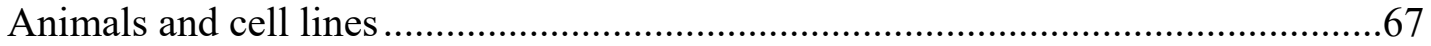

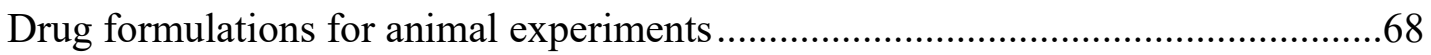

Estimation of ketamine exposure in rat CSF and plasma .........................................68

Pharmacokinetic study of ketamine in WT, Bcrp KO, Pgp KO and dKO mice

after oral and intraperitoneal dosing at $100 \mathrm{mg} / \mathrm{kg}$..............................................68

Determination of ketamine concentration in mouse serum by HPLC/UV .................69

Measurement of ketamine brain/serum ratio following IP ketamine..........................69

Pharmacokinetic data analysis and interpretation.................................................70

Non-compartment analysis ........................................................................ 70

Nonlinear mixed effect modeling .................................................................... 70

Comparison of ketamine-induced duration of Loss of Righting Reflex (dLORR) between WT, Bcrp KO, Pgp KO and dKO mice ......................................................71

Intracellular uptake of radiolabeled ketamine in MDCKII cells overexpressing

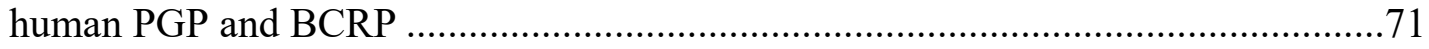

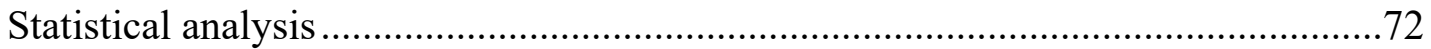

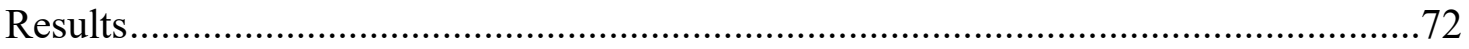

Deletion of both Bcrp and Pgp results in greater ketamine exposure in the CSF and plasma of dKO rats.......................................................................................

Ketamine oral (PO) serum exposure is higher in mice lacking Bcrp and Pgp...........74 Population pharmacokinetic analysis demonstrates an increase in ketamine oral bioavailability in Pgp/Bcrp dKO and Pgp KO mice compared to WT and Bcrp

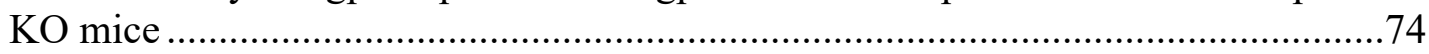
Ketamine-induced duration of loss of righting reflex (dLORR) was significantly increased in mice lacking Bcrp and Pgp. 
Pharmacological inhibition of Bcrp and Pgp by elacridar increased IP ketamineinduced dLORR in WT mice

Ketamine-induced dLORR was significantly longer in dKO vs. WT mice after oral dosing of $200 \mathrm{mg} / \mathrm{kg}$ ketamine

Ketamine accumulation is lower in MDCKII cells transfected with human BCRP

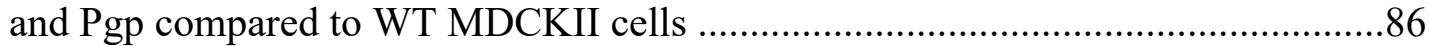

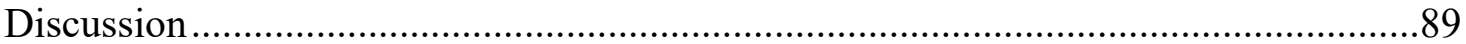

\section{CHAPTER 4. PHEOPHORBIDE A: IN VIVO BCRP SUBSTRATE TO} IDENTIFY ORAL DRUG-DRUG INTERACTIONS..................................................93

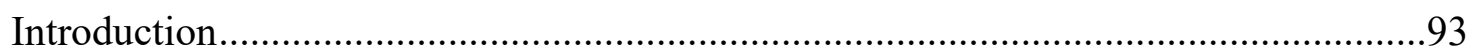

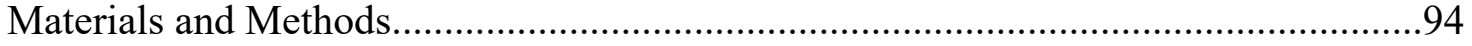

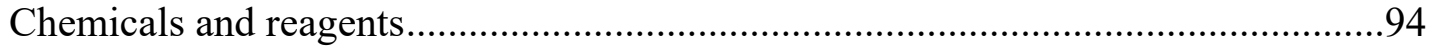

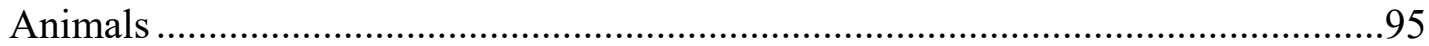

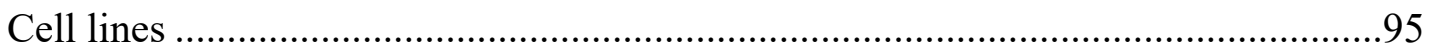

Determination of Pheophorbide A concentration in rodent food pellet......................95

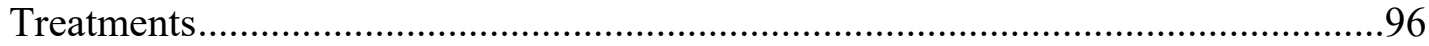

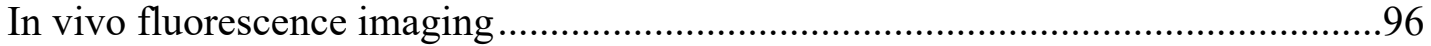

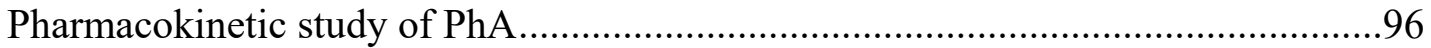

Measurement of PhA concentration in whole serum ............................................96

Ex vivo fluorescence imaging of mouse intestine ...................................................97

Fluorescence microscopy of intestinal sections ....................................................97

In vitro assay for measuring IC50 of Bcrp inhibitors ………..................................97

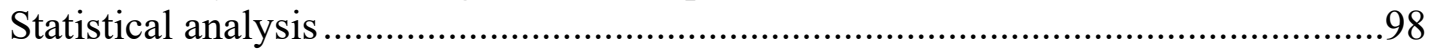

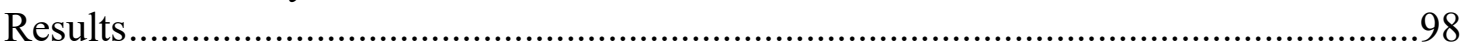

Bcrp KO mice on a regular diet have higher whole-body fluorescence, arising

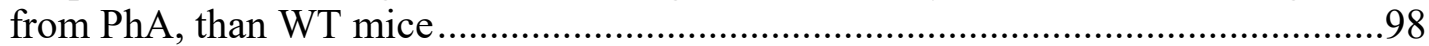

Pheophorbide A is present in regular mouse diet, but not in AFF diet.....................100

Bcrp gene deletion leads to higher absorption of $\mathrm{PhA}$ in mouse enterocytes..........100

Oral absorption of $\mathrm{PhA}$ is greater in Bcrp KO mice treated with exogenous PhA...103

Selected Bcrp inhibitors exhibited comparable in vitro IC50 values with PhA as

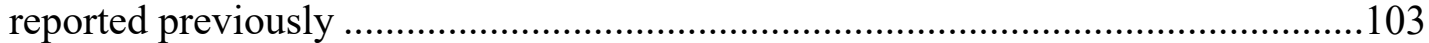

Bcrp inhibitors increase whole body and serum fluorescence of $\mathrm{PhA}$....................107

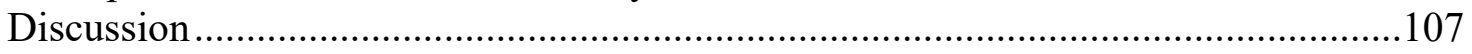

CHAPTER 5. SUMMARY AND DISCUSSION ……...................................................116

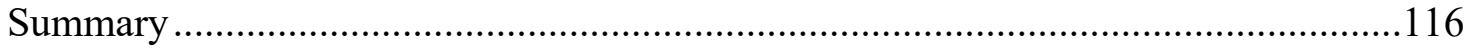

Perspectives and Future Directions.........................................................................117

LIST OF REFERENCES ............................................................................................121

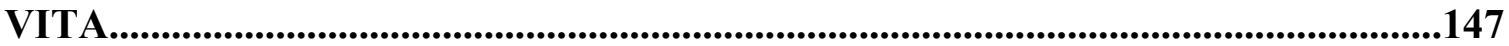




\section{LIST OF TABLES}

Table 2-1. Decrease in fatty acids in dKO rat plasma ..............................................43

Table 2-2. Increase in lysolipids in $\mathrm{dKO}$ rat plasma ...............................................45

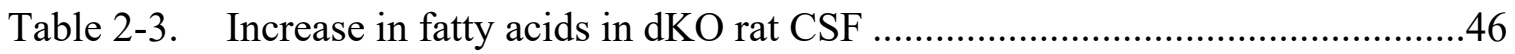

Table 2-4. Gut microbiota metabolites identified altered in the plasma or CSF of

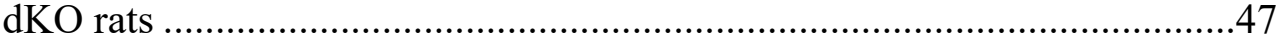

Table 2-5. Sulfated metabolites significantly altered in the plasma of $\mathrm{dKO}$ rats...........48

Table 2-6. Sulfated metabolites significantly altered in the CSF of dKO rats..............48

Table 2-7. Significantly changed Cytochrome P450 (CYP) enzymes in dKO rats .......51

Table 2-8. Significantly changed ATP-binding Cassette $(\mathrm{ABC})$ transporters in $\mathrm{dKO}$

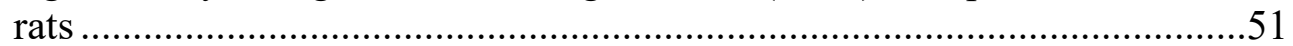

Table 2-9. Significantly changed SLC transporters in $\mathrm{dKO}$ rats...............................52

Table 2-10. Significantly altered top biological pathways based on gene expression changes in $\mathrm{dKO}$ compared to WT rats

Table 2-11. Significantly altered common biological pathways between all the tissues

Table 3-1. Noncompartment analysis (NCA) parameters for ketamine IP and PO

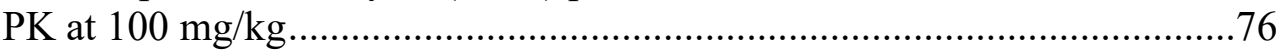

Table 3-2. $\quad$ Population PK parameter estimates .................................................. 81

Table 3-3. Descriptive statistics of ketamine induced dLORR in male FVB mice (WT, Bcrp ${ }^{-/-}$, Pgp $^{-/}$, Bcrp-Pgp ${ }^{-/}$)

Table 3-4. Descriptive statistics of ketamine induced loss of righting reflex (LORR) in male WT FVB mice with or without $100 \mathrm{mg} / \mathrm{kg}$ oral elacridar 1.5 hours before intraperitoneal ketamine dosing....

Table 4-1. Results of all in vitro, in vivo experiments and literature data-based comparisons 


\section{LIST OF FIGURES}

Figure 1-1. Effect of BCRP transporter on its substrate disposition at the Blood Brain Barrier, Choroid Plexus and Arachnoid Barrier.

Figure 1-2. Effect of BCRP and PGP mediated efflux on brain/CSF and blood concentration of its substrates

Figure 1-3. Effect of inhibition or absence of BCRP and PGP on the Brain/CSF and blood concentration of its substrates

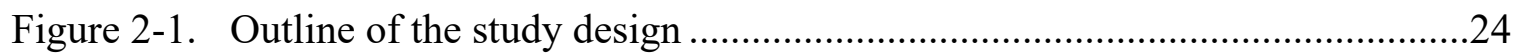

Figure 2-2. Metabolites in CSF and plasma are differentially expressed between WT and Bcrp-Pgp dKO rats (MetaboAnalyst)

Figure 2-3. Metabolites in CSF and plasma are differentially expressed between WT and Bcrp-Pgp dKO rats (Metabolon ${ }^{\circledR}$ )

Figure 2-4. Significantly altered metabolites in CSF and plasma of dKO rats (compared to WT) display unique class signatures

Figure 2-5. Significantly altered metabolites in CSF of dKO rats (compared to WT) display unique class signatures: Volcano plot in CSF

Figure 2-6. Significantly altered metabolites in plasma of dKO rats (compared to WT) display unique class signatures: Volcano plot in plasma.

Figure 2-7. Random forest analysis identifies metabolites that significantly distinguishes between WT and dKO group of rats in CSF

Figure 2-8. Random forest analysis identifies metabolites that significantly distinguishes between WT and dKO group of rats in plasma

Figure 2-9. Independent analysis of metabolome data with MetaboAnalyst identifies similar metabolites, along with known Bcrp substrates in CSF.

Figure 2-10. Independent analysis of metabolome data with MetaboAnalyst identifies similar metabolites, along with known Bcrp substrates in plasma .....

Figure 2-11. Known endogenous substrates of Bcrp were increased in abundance in both plasma and CSF of dKO rats.

Figure 2-12. Identification of metabolites with significant accumulation in CSF of dKO compared to WT rats: Volcano plot

Figure 2-13. Identification of metabolites with significant accumulation in CSF of dKO compared to WT rats 
Figure 2-14. Unlike urate riboflavin did not accumulate in CSF

Figure 2-15. Gene expression in brain, kidney and liver of the dKO is significantly different from the WT rats

Figure 2-16. Possible explanation of observed decrease in corticosterone, a Pgp substrate, in the $\mathrm{dKO}$ rat plasma

Figure 2-17. Possible explanation of activation of peroxisome proliferator-activated receptor pathway in absence of Bcrp and/or Pgp

Figure 2-18. Possible biological pathways altered in the absence of Bcrp and Pgp in the $\mathrm{dKO}$ rats, based on both transcriptomic and metabolomic evidences....57

Figure 2-19. Metabolites with significantly higher abundance in dKO CSF compared to WT rats have structural properties for interaction with BCRP ..

Figure 3-1. Elevated ketamine CSF and plasma levels in Pgp/Bcrp dKO vs. WT rats...73

Figure 3-2. Ketamine serum exposure in Pgp/Bcrp dKO mice is significantly higher than in WT mice after oral dosing... .75

Figure 3-3. Schematic diagram of the final population model . .77

Figure 3-4. Diagnostic plots - observed vs. population and individual predicted ketamine concentrations . .78

Figure 3-5. Diagnostic residual plots

Figure 3-6. Normalized prediction distribution error (NPDE) plots .80

Figure 3-7. The duration of IP ketamine induced loss of righting reflex (dLORR) significantly increases in mice with absent or inhibited Pgp and Bcrp

Figure 3-8. Ketamine brain/serum ratios in wild-type, PgpKO and Pgp/Bcrp-dKO mice

Figure 3-9. ${ }^{3} \mathrm{H}$-ketamine uptake is lower in the MDCKII BCRP-PGP cells compared to MDCKII WT cells.

Figure 4-1. Autofluorescence derived from dietary $\mathrm{PhA}$ is higher in the Bcrp KO compared to WT mice

Figure 4-2. PhA is present in the regular mouse diet not in the AFF diet. 101

Figure 4-3. Absence of Bcrp leads to higher absorption of $\mathrm{PhA}$ in enterocytes 102

Figure 4-4. PhA oral absorption is significantly higher in the Bcrp KO mice 104 
Figure 4-5. Determination of IC50 for selected Bcrp inhibitors using PhA as a probe substrate.

Figure 4-6. Inhibition of BCRP increased whole body fluorescence in control FVB mice orally dosed with $\mathrm{PhA}$..................................................................108

Figure 4-7. Inhibition of BCRP increased whole body fluorescence as well as the serum concentration of $\mathrm{PhA}$ in control FVB mice....

Figure 4-8. Fluorescence spectra showing identity of the $\mathrm{PhA}$ peak used for determination of $\mathrm{PhA}$ concentration in serum..... 


\section{LIST OF ABBREVIATIONS}

$\mathrm{AB}$

$\mathrm{ABC}$

ABCA

$\mathrm{ABCA} 3$

ABCA4

ABCA7

ABCB1

$\mathrm{ABCB} 11$

ABCB4

$\mathrm{ABCC}$

$\mathrm{ABCC} 1$

$\mathrm{ABCC} 2$

$\mathrm{ABCC} 4$

$\mathrm{ABCC} 7$

$\mathrm{ABCD}$

$\mathrm{ABCG}$

ABCG2

ABCG5

ABCG8

ACE

AFF

AMPAR

ATF3

ATF4

ATP

AUC

BBB

BCRP

BCS

BCSFB

BSEP

BSV

Caco-2

cAMP

CCM

cDNA

cGMP

$\mathrm{CHO}$

CLPa

Cmax

CNS

$\mathrm{CP}$

CSF
Arachnoid barrier

ATP-binding cassette

ATP-binding cassette sub-family A

ATP-binding cassette sub-family A member 3

ATP-binding cassette sub-family A member 4

ATP-binding cassette sub-family A member 7

ATP-binding cassette sub-family B member 1

ATP-binding cassette sub-family B member 11

ATP-binding cassette sub-family B member 4

ATP-binding cassette sub-family $\mathrm{C}$

ATP-binding cassette sub-family $\mathrm{C}$ member 1

ATP-binding cassette sub-family $\mathrm{C}$ member 2

ATP-binding cassette sub-family $\mathrm{C}$ member 4

ATP-binding cassette sub-family $\mathrm{C}$ member 7

ATP-binding cassette sub-family D

ATP-binding cassette sub-family $\mathrm{G}$

ATP-binding cassette sub-family $\mathrm{G}$ member 2

ATP-binding cassette sub-family $\mathrm{G}$ member 5

ATP-binding cassette sub-family $\mathrm{G}$ member 8

Angiotensin converting enzyme

Alfalfa-free

$\alpha$-amino-3-hydroxy-5-methyl-4-isoxazole propionic acid receptor

Activating transcription factor 3

Activating transcription factor 4

Adenosine triphosphate

Area under curve

Blood brain barrier

Breast cancer resistant protein

Biopharmaceutical classification system

Blood cerebrospinal fluid barrier

Bile salt export pump

Between-subject variability

Human colon carcinoma cell line

Cyclic adenosine monophosphate

Curcumin

Complementary deoxyribonucleic acid

Cyclic guanosine monophosphate

Chinese hamster ovary

Chlorophyll a

Maximum plasma concentration

Central nervous system

Choroid plexus

Cerebrospinal fluid 


\begin{tabular}{|c|c|}
\hline CYP & Cytochrome P450 \\
\hline Cyp2b1 & Cytochrome P450 2b1 \\
\hline CYP2B6 & Cytochrome P450 2B6 \\
\hline CYP3A4 & Cytochrome P450 3A4 \\
\hline DDI & Drug-drug interaction \\
\hline $\mathrm{dKO}$ & Double knockout (Bcrp-Pgp double knockout) \\
\hline dLORR & Duration of loss of righting reflex \\
\hline DPBS & Dulbecco's phosphate buffer saline \\
\hline DSB & Dasatinib \\
\hline E.Coli & Escherichia coli \\
\hline ECD & Elacridar \\
\hline EDTA & Ethylenediaminetetraacetic acid \\
\hline Em & Emission \\
\hline EMA & European Medicines Agency \\
\hline Ex & Excitation \\
\hline FAD & Flavin adenine dinucleotide \\
\hline FDA & Food and Drug Administration \\
\hline FMN & Flavin adenine mononucleotide \\
\hline FTC & Fumitremorgin $\mathrm{C}$ \\
\hline FVB & Friend virus $B$ \\
\hline GC-MS & Gas chromatography-Mass spectrometry \\
\hline GIT & Gastrointestinal tract \\
\hline GWAS & Genome-wide association study \\
\hline HEK & Human embryonic kidney \\
\hline HMDB & Human Metabolome Database \\
\hline HPLC & High performance liquid chromatography \\
\hline HPMC & Hydroxypropylmethylcellulose \\
\hline IBD & Inflammatory bowel disease \\
\hline IC50 & Concentration for $50 \%$ inhibition \\
\hline IP & Intraperitoneal \\
\hline ITC & International Transporter Consortium \\
\hline KEGG & Kyoto Encyclopedia of Genes and Genomes \\
\hline $\mathrm{Ki}$ & Coefficient of inhibition \\
\hline $\mathrm{KO}$ & Knockout \\
\hline LC-MS & Liquid chromatography-Mass spectrometry \\
\hline LC-MS/MS & Liquid chromatography-Tandem mass spectrometry \\
\hline LC-NMR & Liquid chromatography-Nuclear magnetic resonance \\
\hline LLC-PK & Lilly laboratories cell porcine kidney \\
\hline LLOQ & Lower limit of quantification \\
\hline LORR & Loss of righting reflex \\
\hline LPB & Lapatinib \\
\hline LPS & Lipopolysaccharide \\
\hline $\mathrm{M} / \mathrm{Z}$ & Molecular weight/charge \\
\hline MATE & Multidrug and toxin extrusion \\
\hline MDCK & Madin-Darby canine kidney \\
\hline MDR1 & Multidrug resistance protein 1 \\
\hline
\end{tabular}




\begin{tabular}{|c|c|}
\hline mRNA & Messenger ribonucleic acid \\
\hline MRP1 & Multidrug-resistance associated protein 1 \\
\hline MRP2 & Multidrug-resistance associated protein 2 \\
\hline MRP3 & Multidrug-resistance associated protein 3 \\
\hline MRP4 & Multidrug-resistance associated protein 4 \\
\hline NBD & Nucleotide-binding domain \\
\hline NIR & Near-infrared \\
\hline NMDA & N-methyl-D-aspartate \\
\hline NMR & Nuclear magnetic resonance \\
\hline NR1D1 & Nuclear receptor subfamily 1 group D member 1 \\
\hline OAT & Organic anion transporter \\
\hline OAT1 & Organic anion transporter 1 \\
\hline OAT3 & Organic anion transporter 3 \\
\hline OATP & Organic anion transporting polypeptide \\
\hline OATP1A1 & Organic anion transporting polypeptide $1 \mathrm{~A} 1$ \\
\hline OATP1A2 & Organic anion transporting polypeptide $1 \mathrm{~A} 2$ \\
\hline OATP1B1 & Organic anion transporting polypeptide 1B1 \\
\hline OATP1B3 & Organic anion transporting polypeptide 1B3 \\
\hline OATP2A1 & Organic anion transporting polypeptide $2 \mathrm{~A} 1$ \\
\hline OATP2B1 & Organic anion transporting polypeptide 2B1 \\
\hline OCT & Organic cation transporter \\
\hline OCT1 & Organic cation transporter 1 \\
\hline OCT2 & Organic cation transporter 2 \\
\hline OCT3 & Organic cation transporter 3 \\
\hline OCTN1 & Organic cation/carnitine transporter 1 \\
\hline OCTN2 & Organic cation/carnitine transporter 2 \\
\hline OFV & Objective function value \\
\hline Papp & Apparent permeability \\
\hline PBPK & Physiologically based pharmacokinetic modeling \\
\hline PBS & Phosphate-buffered saline \\
\hline PCA & Principal component analysis \\
\hline PCFT & Proton-coupled folate transporter \\
\hline PD & Pharmacodynamics \\
\hline Per2 & Period circadian regulator 2 \\
\hline PET & Positron emission tomography \\
\hline PGE2 & Prostaglandin E2 \\
\hline PGP & P-glycoprotein \\
\hline $\mathrm{PhA}$ & Pheophorbide A \\
\hline $\mathrm{PK}$ & Pharmacokinetics \\
\hline PLS-DA & Partial least square discriminant analysis \\
\hline $\operatorname{PPAR} \alpha$ & Peroxisome proliferator-activated receptor $\alpha$ \\
\hline $\operatorname{PPAR} \gamma$ & Peroxisome proliferator-activated receptor $\gamma$ \\
\hline PPIX & Protoporphyrin IX \\
\hline PPZ & Pantoprazole \\
\hline $\mathrm{RBC}$ & Red blood cell \\
\hline RFA & Random Forest Analysis \\
\hline
\end{tabular}




\begin{tabular}{|c|c|}
\hline RFC & Reduced folate carrier \\
\hline RI & Retention index \\
\hline RMA & Robust multiarray averaging \\
\hline RNA & Ribonucleic acid \\
\hline $\mathrm{ROC}$ & Receiver operator characteristic \\
\hline ROI & Region of interest \\
\hline Ror $\alpha$ & Retinoid-related orphan receptor $\alpha$ \\
\hline RSS & Remote sensing and signaling \\
\hline RUV & Residual unexplained variability \\
\hline SEM & Standard error of mean \\
\hline SFB & Sorafenib \\
\hline SLC & Solute carrier \\
\hline SLC19A1 & Solute carrier family 19 member 1 \\
\hline SLC21 & Solute carrier family 21 \\
\hline Slc22 & Solute carrier family 22 \\
\hline SLC22A12 & Solute carrier family 22 member 12 \\
\hline SLC22A2 & Solute carrier family 22 member 2 \\
\hline SLC22A5 & Solute carrier family 22 member 5 \\
\hline SLC22A6 & Solute carrier family 22 member 6 \\
\hline SLC22A8 & Solute carrier family 22 member 8 \\
\hline SLC46A1 & Solute carrier family 46 member 1 \\
\hline Slc47 & Solute carrier family 22 \\
\hline SLC47A1 & Solute carrier family 47 member 1 \\
\hline SLC47A2 & Solute carrier family 47 member 2 \\
\hline SLCO & Solute carrier organic anion \\
\hline SLCO1B1 & Solute carrier organic anion transporter family member 1B1 \\
\hline SNP & Single nucleotide polymorphism \\
\hline Th17 & T helper 17 \\
\hline TMD & Transmembrane domain \\
\hline UPLC-MS & Ultra performance liquid chromatrography-Mass spectrometry \\
\hline URAT1 & Urate transporter 1 \\
\hline WT & Wild type \\
\hline ZT14 & Zeitgeber time 14 \\
\hline ZT2 & Zeitgeber time 2 \\
\hline
\end{tabular}




\section{CHAPTER 1. INTRODUCTION}

\section{Membrane Drug Transporters}

Membrane drug transporters are protein molecules in the membranes of cells or cellular organelles where they help in either uptake or efflux of their substrates into or out of the cells, respectively. Transporters are well-known for regulating the disposition of their xenobiotic substrates and a notable few, such as organic anion transporting polypeptide 1B1 (OATP1B1) or solute carrier organic anion transporter 1B1 (SLCO1B1), organic cation transporter 2 (OCT2) or solute carrier family 22 member 2 (SLC22A2), organic anion transporters $1 / 3(\mathrm{OAT} 1 / 3)$ or solute carrier family 22 members 6/8 (SLC22A6/8), breast cancer resistant protein (BCRP) or ATP-binding cassette sub-family G member 2 (ABCG2), P-glycoprotein (PGP) or ATP-binding cassette sub-family $\mathrm{B}$ member 1 (ABCB1) and multidrug and toxin extrusion protein (MATE) or solute carrier family 47 members $1 / 2$ (SLC47A1/2) are recognized by US Food and Drug Administration (FDA) and European Medicines Agency (EMA) for their impact on drug development $[1,2]$. These transporters also function as an integral part of the biological system, and are involved in maintaining metabolic homeostasis and transporting essential or non-essential endogenous metabolites such as amino acids, cofactors, proteins, lipids, secondary bile acids, purines, pyrimidines as well as versatile metabolites contributed by environment, diet or the microbiome [3-7]. For example, organic cation/carnitine transporter 2 (OCTN2) or solute carrier family 22 member 5 (SLC22A5) transports carnitine, an essential metabolite required for the uptake of longchain fatty acids in cells for fatty acid $\beta$-oxidation [8]. In addition, BCRP / ABCG2 transports riboflavin (vitamin B2) [9], from which flavin adenine dinucleotide (FAD) and flavin adenine mononucleotide (FMN) are generated, that are important co-factors for many enzymes [10]. Therefore, polymorphisms / mutations in the membrane transporters are often associated with disease phenotypes such as hyperuricemia (in case of BCRP), inflammatory bowel disease (in case of PGP), macular degeneration (in case of ABCA4) or other metabolic abnormalities [11-13] [14] [15] [16].

Transporters can be passive, transporting substrates following the concentration gradient, or active, transporting substrates against the concentration gradient [14, 17-19]. The active transporters can obtain their energy for working against the concentration gradient from ATP hydrolysis (ATP-binding cassette transporters) or transporting one metabolite/substrate in exchange of another substrate (antiporter) or along with another substrate (symporter / cotransporter) $[14,18]$. In addition, there are ion channels, that can assume open and closed conformations and maintain an ion gradient across the cell membrane, as well as the aquaporins or water transporters, that transport uncharged water utilizing energy from the osmotic gradient $[18,19]$. 


\section{Uptake transporters}

The uptake transporters comprise of the solute carrier (SLC) superfamily. Currently, there are 65 SLC genes, with almost 450 SLC transporters of varying functions (http://slc.bioparadigms.org) and are divided into 4 main categories: cotransporters (transport along with another substrate), exchangers (transport in exchange of another substrate), facilitated transporters (transport along the concentration gradient) and orphan transporters (function has not been determined yet) [14, 19]. Several physiologically important transporters are uptake transporters, such as those transporting choline, amino acid, fatty acid, serotonin, dopamine, glutamine, glucose, and ion exchangers etc. $[14,19,20]$. Polymorphisms in these transporters are associated with multiple disease conditions such as cardiovascular diseases, Crohn's disease, posttraumatic stress, gout, etc. [12, 21-24]. As mentioned before, among the many SLC transporters, OATP or SLCO transporters, the SLC22 group comprising of OCT and OAT transporters and the SLC47 group comprising of MATE transporters are of particular importance for their effect on xenobiotic disposition $[1,25]$. The SLC group of transporters also comprise of Reduced Folate Carrier (RFC) or SLC19A1, and ProtonCoupled Folate Transporter (PCFT) or SLC46A1, both of which transport folate and antifolate chemotherapeutic drugs [26].

OATP uptake transporters. OATP or SLCO or SLC21 gene family transporters are of great interest for their capacity to transport bile salts, steroids, organic anions and several xenobiotics at the intestine (enterocytes) and liver (hepatocytes) [27, 28]. These transporters also have important endogenous functions and altered expression of OATP transporters has been noted in different cancerous tissues as well [29]. While most of the OATP transporters have a direct ortholog in rodents, OATP1A1, OATP1B1 and OATP1B3 are only found in humans [27]. OATP1B1 and OATP1B3 are liver-specific transporters, while OATP1A2 has the highest expression in blood-brain barrier (BBB) and is also expressed in intestine, and OATP2B1 is expressed ubiquitously in almost all tissues $[27,29]$. OATP transporters, especially OATP1B1 and OATP1B3, as well as OATP1A2 and OATP2A1, have very wide substrate-recognition capacity, with a large number of drugs / xenobiotics being their substrates as well as inhibitors [28, 30]. Significant polymorphism of OATP transporters and their impact on xenobiotic metabolism and disposition in humans has been recognized by the International Transporter Consortium (ITC) [13]. Due to their significant impact on xenobiotic pharmacokinetics (PK) and a large amount of substrate and inhibitor overlap with other transporters and drug metabolizing enzymes, OATP1B1 and OATP1B3 have also been recognized by FDA as important to be considered during drug development, for the assessment of drug-drug interactions (DDIs) when dosed concomitantly with an inhibitor $[1,25]$. Numerous publications have documented such interactions and guidelines, and reports by the ITC are of great interest in this regard [25, 31].

Organic anion and cation transporters. SLC22 group of transporters are most diverse in nature as they comprise of both organic cation (OCT) and organic anion (OAT) transporters. Organic anion transporters comprise of OAT1-10 and the uric acid transporter URAT1, encoded by the SLC22A12 gene. Although mainly present at kidney 
proximal tubules, expression of these transporters has been found in other tissues as well such as liver, placenta, olfactory epithelium, choroid plexus and blood-brain barrier [20]. These transporters play a significant role in transporting diverse endogenous metabolites and xenobiotics of different chemical and therapeutic classes such as amino acids, microbiome metabolites, fatty acids, angiotensin converting enzyme (ACE) inhibitors, diuretics, antibiotics and statins [7, 20]. URAT1 is the major transporter of uric acid and loss of function polymorphism in this transporter is associated with hyperuricemia and gout $[32,33]$. Both OAT1 and OAT3 are recognized by FDA as transporters important to be considered during drug development for substrate or inhibitor liability for the new chemical entity [1].

Organic cation transporters of the SLC22 family are the OCT1-3, OCTN1 and OCTN2 transporters [5]. The SLC47 family or MATE transporters are also cation transporters. While SLC22 are uptake transporters, MATE family transporters SLC47A1 and SLC47A2 are cation antiporter efflux pumps [5]. OCTs are mainly present in the kidney and liver, but expression is also noted in lung, placenta and intestine [27]. Although these transporters are involved in the transport process of multiple important endogenous metabolites including neurotransmitters, hormones and purine metabolites, a knockout mouse model of these transporters does not present with apparent physiological abnormalities, possibly due to a large amount of substrate overlap between the SLC group of transporters [5]. Due to their significant effect on xenobiotic transport and a diverse chemical class of drugs being their inhibitors, MATE1 and 2, as well as OCT2 are FDA-recommended important transporters to be considered for drug development purposes $[1,25]$.

\section{Efflux transporters}

The efflux transporters comprise of the ATP-bound cassette (ABC) transporters that transport their substrates against the concentration gradient by utilizing the energy from ATP hydrolysis [34]. In the mammalian system, there are 49 ABC genes (with 21 pseudogenes) encoding 7 subfamilies of ABC transporters (A-G) [18]. Currently there are 35 mammalian $\mathrm{ABC}$ transporters with assigned functions as endogenous and/or xenobiotic transporters $[14,34]$. ABC transporters are also important for the microbial system and are often associated with treatment resistance in antibacterial therapy $[35,36]$. Bacterial ABC transporters display striking structural similarity with those in the mammalian system, raising the possibility that they are important for host-microbiome interactions $[17,35]$. The major human $\mathrm{ABC}$ drug efflux transporters are ATP-binding cassette subfamily B member 1 (ABCB1) or multidrug resistance protein 1 (MDR1) or Pglycoprotein (PGP), ATP-binding cassette subfamily C member 2 (ABCC2) or multidrug-resistance associated protein 2 (MRP2), ATP-binding cassette subfamily C member 4 (ABCC4) or multidrug-resistance associated protein 4 (MRP4) and ATPbinding cassette subfamily G member 2 (ABCG2) or Breast Cancer Resistance Protein (BCRP) [14, 34]. 
Structure of ABC transporters. The ABC superfamily of transporters have transmembrane domains (TMDs) and nucleotide binding domains (NBDs). The NBDs comprise of the well-conserved Walker A and Walker B motifs. The TMD is membranebound is usually more variable in nature and contains 6 membrane-spanning loops, while the NBDs are more conserved and sit in the cytosol $[14,17,18]$. Most of the ABC transporters contain two NBDs and two TMDs, hence termed as "full" transporters. But ABCG and ABCD family transporters contain only one TMD and one NBD and are thus termed "half-transporters" and require homodimerization (ABCG2) or heterodimerization (ABCG5 and ABCG8) to become functionally active [14, 37].

Clinical significance of $\mathbf{A B C}$ transporters. The mammalian $\mathrm{ABC}$ transporters are of immense physiological importance, known to transport diverse chemical classes of compounds [38] and loss of function mutation in these transporters are associated with several diseases [14, 18, 34]. For example, the ABCA group of transporters are wellknown cholesterol transporters, and a loss of function mutation is known to cause defective cholesterol and phosphatidylcholine transport (Tangier disease) [39]. In addition, $\mathrm{ABCA} 3$ is related to surfactant transport in the lung and is associated with respiratory distress syndrome [40]; ABCA4 is a retina-specific transporter and loss of function of this transporter is associated with early-onset macular degeneration and loss of vision (Stargardt disease) [41]; and the ABCA7 transporter has been recently associated with Alzheimer's disease [42]. While ABCB1 or PGP is a multidrug transporter, ABCB4 transports phosphatidylcholine [14] and $\mathrm{ABCB} 11$ or BSEP (bile salt export pump) transports glycochenodeoxycholic acid, and loss of function of ABCB11 is associated with intrahepatic cholestasis [43]. ABCC group of transporters are multidrug efflux transporters as well and also transport important endogenous metabolites, such as cyclic adenosine monophosphate (cAMP), cyclic guanosine monophosphate (cGMP), uric acid and bilirubin [14]. A genome-wide association study of Kawasaki disease patients has suggested a possible role of ABCC4 transporter in this disease [44]. Mutations in ABCC7 are known to be associated with cystic fibrosis disease [45]. ABCG group of transporters are cholesterol transporters and loss of function mutation in ABCG5 or ABCG8 is associated with sitosterolemia [37].

$\mathrm{ABC}$ efflux transporters are present in different tissues and organelles, and their effect on drug transport has been mostly studied at the epithelial or endothelial cells of different tissues and blood vessels, bile canaliculi, kidney tubular cells, blood brain barrier (BBB) and the enterocytes [14, 34, 38]. Efflux transporters, such as ABCB1 or PGP, ABCG2 or BCRP, ABCC1-4 or MRP1-4 are well known for their effect on the pharmacokinetics and/or pharmacodynamics and also toxicity and/or side effect of their substrates [38, 46] [47]. A significant impact of Pgp transporter is observed in regulating the brain permeability of its substrates and thereby reducing the toxicity due to unwanted brain exposure of toxic chemicals, such as ivermectin, in mice [48]. Pgp present at the BBB endothelial cells, effluxes its substrates and limits their permeability in brain. Therefore, lack of Pgp function at the BBB often shows a dramatic increase in brain exposure of its substrate drugs, e.g., digoxin and vinblastine [49, 50]. Also, of interest, it has been previously noticed that Pgp knockout (Pgp KO) mice develop a pathological 
condition similar to inflammatory bowel disease (IBD) [51] and a polymorphism of MDR1 or PGP has been recently associated with IBD in humans as well [16].

Endogenous substrates of ABC transporters. As briefly discussed before, the $\mathrm{ABC}$ efflux transport system has important physiological functions and thus are often associated with pathological conditions [35]. ABCA and ABCG class of transporters are mainly cholesterol transporters $[14,18]$, with specialized function for ABCA4, transporting N-retinylidene-phosphatidylethanolamine at the retina [52]. ABCG class of transporters are reported to transport choline phospholipids and ABCG2 in this subfamily is a multidrug transporter with important endogenous substrates such as uric acid [53, 54]. ABCB subfamily transporter ABCB1 or PGP has been studied extensively for its effect on xenobiotic transport, treatment resistance and unwanted toxicity at the distributing tissues $[38,46,48-50,55,56]$, but also known to transport glucocorticoids [57], endogenous opioids [58] etc. ABCB11 or Bile Salt Export Pump (BSEP) is an important bile salt transporter [43]. ABCC subfamily transporters have great physiological significance, transporting prostaglandins, leukotrienes, conjugated bilirubin, cAMP, cGMP and uric acid among many important endogenous substrates [59, $60]$.

Efflux transport and xenobiotic substrates. The ABC efflux transporters are well-known to transport a wide variety of xenobiotics as their substrates, such as chemotherapeutics, immunosuppressants, antibacterial, antiviral, etc. [3, 6, 59]. Identification of several efflux transporters is related to their drug resistance in cancer treatment, and their role is much appreciated now as transporters of relatively broad chemical specificity $[6,38,46,61-63]$. Certain structural or physicochemical features have been identified to be preferred than others for substrate specificity, which are thereby utilized to formulate a structure-property-based approach to identify substrates and inhibitors of these transporters. For example, in case of PGP, pharmacophore and machine-learning based approaches have identified newer inhibitors [64-66]. Some structural features for PGP transporter substrates are aromatic hydrophobic rings, uncharged or weak basic molecules, but often amphipathic in nature [38]. Similar to PGP, another promiscuous transporter ABCG2 inhibitor/interaction identification has also been approached via ligand-based computational methods and were further confirmed by cellbased studies, as well as cell-based screening methods utilizing diverse structural classes of molecules [67, 68]. BCRP substrates tend to have aromatic hydrophobic rings, with a large number of $\mathrm{H}$-bond donors such as nitrogen residues and sometimes display weak acidic properties [69]. Although PGP has been observed to have broad substrate specificity [70], other transporters often display some structural preferences. For example, the MRP group of transporters usually transport amphiphilic organic anions and glutathione-conjugated cations [59]. Further information specifically about the BCRP and PGP transporters have been detailed later in this Chapter. 


\section{Drug-drug interactions and transporter/enzyme substrate overlap}

Drug transporters are known to share common substrates and inhibitors with other transporters as well as drug metabolizing enzymes. Many drugs such as rifampicin, cyclosporin and tyrosine kinase inhibitors interact with multiple drug transport systems as well as enzymes [71]. This may result in clinical pharmacokinetic drug-drug interactions (DDIs) which can further lead to undesired over-exposure to a drug that is concomitantly dosed with a medication which is an inhibitor of a transporter or enzyme system [72]. Besides inhibition of an enzyme by competitive or noncompetitive mechanisms, transporters and enzymes may also be regulated by direct or indirect mechanisms. For example, chemotherapeutic kinase class of drugs and protein-protein interactions affect the expression of $\mathrm{ABC}$ drug transporters as well as organic cation transporters, leading to possible DDIs [73-75]. Apart from interaction with a co-treatment, significant clinical interactions have also been reported for dietary and natural compound transporter and/or enzyme inhibitors such as St. John's wort and grapefruit juice among several others [7679]. These interactions have often been identified and studied extensively, thereby leading to distinct guidelines by the regulatory agencies $[1,80]$.

It is important to recognize that drug transporters and metabolizing enzymes are the major clearance mechanism of a foreign chemical entity in our system. Therefore, major drug metabolizing enzymes and transporters often co-exist in major barrier tissues, such as intestinal epithelial cells, hepatocytes and kidney [81-83]. Drug metabolizing enzymes are less frequent in the blood-brain barrier, kidney and lung, [84] where the

major clearance mechanism is mediated by the transporters. Hence, often, the substrate of a metabolizing enzyme is also a substrate of the transport machinery, such as the statin class of drugs which are substrates of multiple transporters as well as metabolizing enzymes and prone to clinical drug-drug interactions [85]. Many drugs are also inhibitors of multiple transporters, e.g., tyrosine kinase inhibitor lapatinib inhibits BCRP, PGP and URAT1 [71], elacridar inhibits PGP, BCRP as well as OATPs [86, 87], etc. Therefore, a complete understanding of determinants of drug pharmacokinetics is important, with the identification of at least the major enzymes and transporters in different tissues of interest.

\section{Assays for identification of transporter substrates and inhibitors}

In vitro assays. Identification and/or confirmation of substrates or inhibitors of transporters are often performed in vitro and several assay systems have been developed for such purposes. The White papers from ITC provide a great guideline on data analysis, usefulness and advantages or disadvantages of these systems [88, 89]. The following four types of in vitro transporter assays are usually widely used:

1. Membrane vesicles: An inside-out membrane vesicle system is used for the identification of efflux transporter substrates, as well as inhibitors. In this case, an analytical method capable of detecting low concentration of the metabolite is required, or else, fluorescent or radioactive materials are used for these assays. Meaningful kinetic 
properties of the transporter-substrate interaction are obtained from these assays. For highly lipophilic and diffusible compounds, binding to the vesicles may lead to false negative results [88].

2. Recombinant cell lines: Stable transfection of host mammalian cells with fulllength transporter cDNA is used for generation of different transporter cell lines. Human embryonic kidney (HEK), canine kidney (MDCK), Chinese hamster ovary (CHO) and porcine kidney (LLC-PK) cells are among the many cell lines that are used for transporter substrate identification. MDCK and LLC-PK cells usually grow faster and form polarized monolayer with tight junctions and are thereby very suitable for transwell permeability studies and uptake / efflux assays for substrate or inhibitor identification. Changes in cell viability in presence of a transporter inhibitor and a cytotoxic substrate (e.g. mitoxantrone) can also be used for inhibitor identification. Although used as an indirect proof of substrate, the inhibitory effect of a compound on transporter substrate accumulation or transport is mainly a proof of interaction and should be used with caution, since many transporter inhibitors were eventually not found to be substrates. Moreover, presence of endogenous transporters should also be noted for such assays. In addition, multiple cell culture variables need to be kept in mind when developing or running the assays, such as culture time, passage number, media component (albumin content) etc. [88]. Use of multiple transporter substrates or inhibitors as positive controls are recommended by ITC and the regulatory agencies which also detail the methods for data analysis $[88,89]$.

3. Bidirectional assays: Bidirectional assays utilizing recombinant cell lines (LLC-PK, MDCK or Caco-2) is the recommended system by the regulatory agencies for identification of transporter substrates and inhibitors [1,88]. A transwell format is used where cells are grown on a transwell insert, the substrate is added on either basolateral or apical side of the chamber (donor compartment) with or without the inhibitor, and concentration of the substrate transported to the other side of the chamber (receiver compartment) is measured. Different analytical methods can be utilized to calculate the final outcome to measure apparent permeability (Papp) values that can be utilized in vivo in different PBPK models incorporating transporter kinetics. ITC provides a thorough guideline for execution and data analysis of such assays [89, 90]. Presence of endogenous transporters is a factor in this assay as well. Moreover, compounds with low permeability might be difficult to study in this assay [88].

4. Hepatocytes: The hepatocyte culture system is used to study in vitro uptake, efflux and metabolism of xenobiotics in the liver. Suspension-cultured and sandwichcultured hepatocyte systems exist, with a preference for the later, as it mimics closely the complicated network in human liver and creates the biliary duct system in vitro in a 3D cell culture model. This is a very complex system and use of specific inhibitors are required to tease out the effect of each transporter or enzyme on the transport or metabolism of the compound tested. But due to its closeness to the physiological complexity, this method has a high translational relevance [88]. 
Apart from ITC, the regulatory agencies also provide a detailed guideline on how to use in vitro assays for identification of transporter substrate or inhibitors and their utilization for identification of DDIs [1].

In vivo models. Transporter knockout models as well as inhibitor-based studies in mice and rats have been utilized for substrate identification [48, 91, 92]. Studies with inhibitors are required to confirm the findings in the knockout models to rule out the effect of compensatory pathway effects. Although prototypical Bcrp and Pgp substrates are known to have increased brain permeability in absence of the transporters at the blood-brain barrier [50,93, 94], caution must be taken to avoid misinterpretation of results for high permeability drugs that may not show increased accumulation in brain in a knockout model, but whose pharmacokinetics and pharmacodynamics are still affected by the transporters [95-98]. Apart from the knockout models, noninvasive powerful imaging techniques such as positron emission tomography (PET) and whole-body autoradiography may also be used with carefully designed probes for identification of transporter substrates and/or inhibitors, or even to understand the pharmacodynamics of a drug $[99,100]$. Another technique used is gamma-scintigraphy that utilizes gammaemitting isotopes such as ${ }^{99 \mathrm{~m}} \mathrm{Tc}$ to assess drug disposition. However, these methods are expensive and have their own practical limitations as well [25].

\section{BCRP and PGP Efflux Transporters}

$\mathrm{BCRP}$ or $\mathrm{ABCG} 2$ and $\mathrm{PGP}$ or $\mathrm{ABCB} 1$ are two major drug efflux transporters, which are present at major drug absorption and/or elimination sites as intestine, liver, kidney, blood brain barrier, and their major function is to reduce systemic exposure of their substrates.

\section{BCRP efflux transporter}

Breast cancer resistant protein (BCRP) or ABC sub-family G member 2 (ABCG2) is a half transporter, as it contains two subunits of one TMD and one NBD domain, that requires homodimerization to become active [14]. BCRP is a $72 \mathrm{kDa}$ protein with 665 amino acids and is highly conserved across the mammalian as well as vertebrate species, suggestive of its conserved function across all species [101, 102]. Using BXP-34 and BXP-21 antibodies, Maliepaard et al. were the first to provide an extensive report of BCRP expression in normal human tissues [103], showing high expression in the placental syncytiotrophoblast, the small intestinal epithelium, the canalicular membrane in liver and in the duct and lobules of breast. Interestingly, it was found to have high expression in vein endothelium but not in the arteries. Expression of BCRP was later confirmed at choroid plexus and blood-brain barrier in the brain, kidney proximal tubules and cardiomyocytes among several other tissues [102, 104]. Expression of BCRP has been extensively studied in human intestine and found to have a high expression in the duodenum with decreasing expression further down the gastrointestinal tract [105]. Other important expression compartments of BCRP include the normal hematopoietic stem 
cells $[106,107]$ as well as cancer stem cells [108]. Although its expression is not required for having stem cell-like properties, BCRP expression on the stem cells (side population cells) help in their identification [108-110].

Clinical significance of BCRP. BCRP transporter polymorphisms Q141K and V12M are well-characterized, with the loss of function polymorphism Q141K exhibiting a high frequency of occurrence in the Asian population [111-113]. Several other important polymorphisms, such as Q126X, in this transporter have also been noted [11, 114]. BCRP polymorphism has also been associated with pathological conditions such as gout [24], apart from its effect on the oral bioavailability of its substrates such as topotecan [115]. Of particular interest, Matsuo et al. showed a positive correlation of BCRP polymorphism with gout, but negative correlation with Parkinson's disease [116]. In addition, due to BCRP-mediated transport of drugs, it has been associated with several treatment-resistant diseases, especially cancer [117, 118]. BCRP polymorphism has also been associated with a $\operatorname{Jr}(\mathrm{a}-)$ blood group phenotype in which the affected individuals lack a $\operatorname{Jr}(\mathrm{a})$ antigen on their red blood cells, thereby making them prone to transfusion reaction or hemolytic disease $[119,120]$.

Xenobiotic substrates or inhibitors of BCRP. A diverse group of compounds, belonging to different chemical classes, such as anthracene drugs (mitoxantrone, bisantrene), camptothecin (topotecan), nucleoside analogues (zidovudine), statins (rosuvastatin), tyrosine kinase inhibitors (gefitinib, sorafenib), proton pump inhibitors (pantoprazole) and fluoroquinolone antibiotics are substrates of BCRP transporters [6, $121,122]$. Apart from in vitro studies, the mouse Bcrp KO model has also been utilized to determine substrates and inhibitors of Bcrp. Mouse Abcg2 or Bcrp has $81 \%$ protein sequence similarity with human ABCG2 or BCRP [55], and mutation in the amino acid at 482 position affected substrate specificity in both species [123]. Therefore, substrate and inhibitor profile of BCRP in humans could be compared to that in mice. Extensive reviews articles have been published with a detailed list of substrates and inhibitors of BCRP [6] [102] [121], with new molecules being added as well.

Endogenous substrates of BCRP. BCRP is also known to play an important role in transporting endogenous and dietary substrates. Endogenous metabolites uric acid, riboflavin, folic acid and protoporphyrin IX have been reported as substrates of BCRP transporter [9] [54] [114] [124] [92]. BCRP is also associated with heme transport [109] [125], though a direct evidence of heme transport by BCRP does not exist yet. In addition, dietary chlorophyll catabolite Pheophorbide A was found to be a highly selective BCRP substrate [92], and many gut microbiota-derived uremic toxins and metabolites are known to interact with BCRP as well [126] [83]. BCRP has also been shown to have a high affinity for sulfated metabolites, sulfated steroid conjugates as well as phytoestrogen sulfates [127] [128] [129].

Physiological significance of BCRP. BCRP transporter expression at the enterocytes and blood-brain barrier (BBB) as well as blood CSF barrier (BCSFB) has been shown to limit oral bioavailability and brain permeability of its substrates. At the bile canaliculi and kidney proximal tubules, BCRP increases the clearance of its 
substrates and is known to protect the fetus from undesired chemical exposure at the placental barrier [6] [105, 130] [104]. While absence or inhibition of BCRP has been shown to increase the brain penetration of its substrates [94] [131], previously published reports also show that inhibition or absence of Bcrp at the choroid plexus (CP) epithelial cells leads to reduced ventricular CSF concentration of Bcrp substrate topotecan in mice [132] [133], whereas the brain concentration of topotecan still increases.

Immunohistochemical labeling of Bcrp using BXP-53 antibody demonstrated that Bcrp is present on the apical membrane of choroid plexus epithelial cells, facing the CSF [133]. This compartment-specific effect of Bcrp in the mouse brain can also be observed for another Bcrp substrate/inhibitor, lapatinib. A tissue distribution study using ${ }^{14} \mathrm{C}$ labeled lapatinib exhibited higher lapatinib radioactivity in CSF compared to brain in rats [134]. This highlights the physiological role of BCRP as an efflux transporter at BCSFB, effluxing its substrates into CSF. This is further strengthened by the fact that high concentration of uric acid (a BCRP substrate) in serum is negatively associated with Parkinson's disease, but positively associated with gout [116]. Results published by Yasuda $\mathrm{K}$ et al. demonstrated that Bcrp is also present at the arachnoid barrier epithelial cells, part of the BCSFB, both on the apical as well as basolateral side [135]. On the apical side, it faces the blood and the normal function would be regulating blood-to-brain permeability of its substrates, whereas at the basolateral side, it faces the CSF, and therefore probably effluxes its substrates that are important for the metabolic content of CSF (Figure 1-1).

BCRP-mediated transport / interaction with heme has been reported to provide hypoxic cell survival [109, 125]. In addition, its presence on cancer stem cells [129] might further explain the treatment resistance observed in cancer therapy. Apart from cancer and hematopoietic stem cells, BCRP is also found to be expressed in cardiac stem cells and cardiomyocytes and reported to be involved in ischemic cardiac diseases as well $[110,136,137]$.

Uric acid is a well-known BCRP substrate [114] and BCRP has been found to play an important role in the extrarenal or intestinal secretion of uric acid [138]. Hosomi et al. also reported that treatment with Bcrp inhibitor elacridar can reduce the intestinal secretion of uric acid [138]. In this regard, it has recently been noted that intestinal microbiota in gout patients is significantly different compared to the normal population [139]. Gout patients related to BCRP polymorphism are known to secrete less uric acid in the intestine [114]. While Guo et al. did not investigate the presence of BCRP polymorphism in their studied gout patient population, it is important to understand the effect of BCRP, if any, in this context.

While multiple studies have been performed to identify BCRP substrates and inhibitors, including endogenous substrates, a systemic effort to understand the physiological role of BCRP transporter and the consequences of its absence or inhibition by different xenobiotic/dietary inhibitors is still lacking. Due to the effect of multiple transporters on the endogenous metabolites, an investigation of a single metabolite or known metabolic signatures, coupled to a single transporter perturbation system, may 


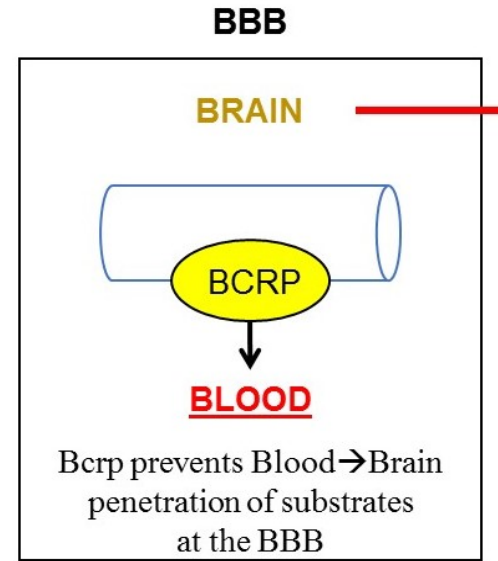

Bcrp substrates

(from systemic circulation)

would be higher in CSF of KO

rats
CP

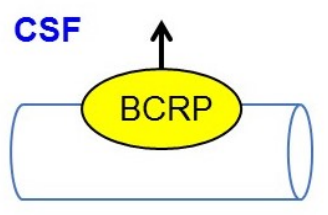

BLOOD

Bcrp facilitates Blood $\rightarrow$ CSF penetration of substrates at the $\mathrm{CP}$

Bcrp substrates (produced in CP cells) might be lower in CSF of KO rats
$A B$

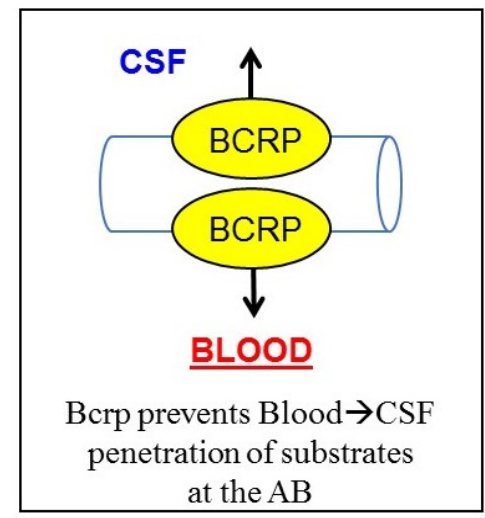

Bcrp substrates

(from systemic circulation) would be higher, but those produced in $\mathrm{AB}$ cells might be lower in CSF of KO rats

Figure 1-1. Effect of BCRP transporter on its substrate disposition at the Blood Brain Barrier, Choroid Plexus and Arachnoid Barrier

Note: BBB: Blood Brain Barrier; CP: Choroid Plexus; AB: Arachnoid Barrier; CSF:

Cerebrospinal Fluid 
provide limiting and often misleading information. The recently developed remote sensing and signaling hypothesis [20,140-142] with regard to OAT transporters is of particular note in this context. Therefore, a global or systemic approach needs to be undertaken to unravel the mystery of physiological interactions and functions of BCRP transporter.

Moreover, an understanding of the endogenous metabolites of BCRP might help identify an endogenous biomarker of this transporter, which might provide further information for identification of clinical DDIs, as well as identification of clinical conditions involving change in expression of BCRP.

\section{PGP efflux transporter}

Compared to BCRP transport system, P-glycoprotein (PGP) or ATP-binding cassette subfamily B member 1 (ABCB1) has been studied more extensively and its clinical significance with respect to drug disposition is better understood [46]. ABCB1 is a $170 \mathrm{kDa}$ protein and a "full transporter" in that it contains 2 TMDs containing 12 membrane-spanning loops and 2 NBDs. [143]. Impact of PGP on the brain penetration of its substrate drug was accidentally noted first with ivermectin, leading to a 100 -fold higher neurotoxicity in the Pgp KO mice [48]. Later the effect of PGP transport system was investigated on the brain penetration of multiple xenobiotics such as digoxin, cyclosporine, dexamethasone and tyrosine kinase inhibitors such as dasatinib and ponatinib [50] [144] [143]. PGP is found to be expressed in several tissues and organs, mainly providing protective function from exogenous toxins / chemicals [46] [70]. It has high expression at the intestinal epithelial cells (reducing oral absorption of its substrates), canalicular cells at the hepatocytes (increasing biliary excretion), kidney (increasing urinary elimination) and placenta (protection of the fetus) among many other tissues [56]. Expression of PGP in the human liver was reported to be lower than that in enterocytes, and hepatocyte expression was found to have almost 50 -fold inter-individual variability $[143,145]$. Recently pro-inflammatory Th17 cells in intestine were found to express PGP and were refractory to glucocorticoid therapy [146], raising the possibility that Pgp might be involved in maintaining normal colorectal physiology [15].

Clinical significance of Pgp. Multidrug resistance in cancer cells was identified as early as 1986 by Shen et al. [63] which was later linked to PGP [147], and its expression in normal human tissues was established as well [148]. Since then, multiple studies have established the clinical importance of PGP in chemo-resistance, resistance to anti-HIV therapy and its effect on xenobiotics of diverse chemical and therapeutic classes [38] [149] [46] [3]. Pgp is expressed at the BBB and affects the brain permeability of its substrates, thereby limiting efficacy or increasing toxicity risk in case of DDIs [150] [151] [48]. Lack of Pgp function has also been noted to cause IBD-like conditions in animal models [16], raising the concern that this transporter might have an important role in the gastrointestinal tract [51]. A large number of single nucleotide polymorphism (SNPs) in the coding region of PGP has been observed, leading to decreased mRNA or sometimes protein expression of the transporter, but a significant impact on the drug 
pharmacokinetics or pharmacodynamics is yet to be established [70]. Moreover, in several disease conditions, especially in central nervous system (CNS) diseases such as epilepsy [152] [153] [154], expression of PGP can change, leading to a possible increase in PGP substrate exposure in the CNS [155].

Xenobiotic substrates or inhibitors of PGP. Due to its effect on chemoresistance, and on the disposition of a large number of therapeutic class of compounds such as antibiotics, immunosuppressants, anticancer and cardiovascular drugs etc. [156], researchers have been looking for PGP inhibitors with higher specificity and clinical usefulness [38]. At least 3 generations of PGP inhibitors exist based on their chemical classification, specificity and toxicity properties and several comprehensive lists of inhibitors have been published [157] [38]. While the first generation of inhibitors were cytotoxic (verapamil) or nonspecific inhibitors of multiple transporters or enzymes (pantoprazole), PSC833 or valspodar belong to the second generation, and the third generation comprises of elacridar and similar drugs such as zosuquidar and tarquidar, which have been developed to be used in the clinic [158].

Endogenous substrates of PGP. Although the effect of PGP on xenobiotic substrates is well established, the endogenous function of this transporter is not well understood. PGP has been reported to transport endogenous opioids such as adrenorphin, endomorphin 1 and 2, neurokinin [58], glucocorticoids such as cortisol and aldosterone, [159] [57] and possibly bilirubin [160]. In addition, using conjugated phosphocholines and other short chain fatty acids, it was shown that PGP is a broad specificity lipid translocase [161].

Physiological significance of PGP. The physiological significance of PGP transporter is mainly assigned to its protective function at enterocytes and BBB, and its importance as a broad specificity chemical transporter, with the possibility of lipid translocation and of other known endogenous substrates $[3,70]$. The absence of Pgp had been reported to express an IBD like symptoms [16] along with a change in gut microbiome [162] [51], and association with colorectal carcinoma and ulcerative colitis is also suggested [163] [15]. Expression of Pgp on the glucocorticoid treatment-resistant Th17 cells in intestine [146] also points towards the important role that Pgp might play in regulating intestinal health or even on the microbiome homeostasis. Alteration in Pgp expression at the BBB due to pathological conditions [153], effect of amyloid beta protein on the expression of Pgp [164] at the BBB and the significant role of Pgp at the $\mathrm{BBB}$, suggest some important endogenous functions of this transporter. Understanding of the endogenous function of PGP and the effect of its expression changes, especially at the $\mathrm{BBB}$, might be extremely helpful in avoiding serious DDIs. Identification of a possible biomarker might also be helpful in assessing the functional level of the transporter in the system. 


\section{Substrate or inhibitor overlap of BCRP and PGP}

Due to their broad substrate specificity, BCRP and PGP transporters show a significant overlap in their substrate and/or inhibitors, with differences in affinities for either transporters [102]. Several tyrosine kinase inhibitor class of drugs are substrates/inhibitors of both the transporters [165] [166] [134], with many other xenobiotic inhibitors, such as pantoprazole and elacridar displaying similar properties as well [71]. This could be particularly useful for drug permeability in difficult-to-treat tissues, such as brain tumor. It has often been found that both BCRP and PGP expression plays an important role in controlling brain concentration of their substrates [167]. Therefore, dual substrates of Bcrp and Pgp transporters are well studied in literature due to the dramatic effect on their brain permeability in absence of these transporters in rodents. For example, a 25 -fold increase in brain permeability of ponatinib, a tyrosine kinase inhibitor, is observed in female mice in absence of Bcrp and Pgp [144]. In mice, Pgp has a much higher expression at the blood-brain barrier than Bcrp [168], leading to dramatic increase in the brain permeability of Pgp substrates. For example, a 35-fold increase in brain permeability of digoxin is observed at 4 hours after its intravenous injection in mice [50]. But in higher mammals, such as monkeys, dogs and humans, BCRP and PGP are expressed in almost similar abundance [168, 169]. In dog brain capillaries, BCRP expression has been reported to be remarkably higher than PGP [170], whereas it was not detected at the choroid plexus. Therefore, the effect of BCRP could be underestimated from preclinical study, than what would be observed in the clinic. On the other hand, Uchida et al. reported a higher PGP than BCRP expression at the choroid plexus in humans, but are almost the same in rats [171]. Moreover, Pgp expression at the choroid plexus in mice has not been reported yet and is controversial in literature [133, 135, 172-174]. This limits the usefulness of knockout mouse models for observations related to brain concentrations of BCRP and PGP substrates and specifically their effect on the CSF concentration of their substrates.

Due to their affinity for compounds from similar Biopharmaceutical Classification System (BCS) class and their significant expression at enterocytes, BCRP and PGP are expected to have a significant impact on the oral absorption of their substrates $[1,25,175]$. Moreover, often, the inhibitors may not achieve sufficiently high systemic concentrations to display the systemic inhibition of these transporters [71]. Therefore, except for the loss of function polymorphism related cases, systemic inhibition and related overexposure of BCRP and/or PGP substrates are not so frequent. Hence, the meaningful clinical impact of these transporters is their effect on oral absorption. Still, caution must be taken for compounds such as lapatinib, which has high hydrophobicity, and therefore can have high tissue distribution and sufficient concentration to impart systemic inhibition of the transporters [134].

Overall, BCRP and PGP transporter function causes a net decrease in permeability, thereby reducing abundance or exposure of their substrates in plasma and brain/CSF. Therefore, absence or inhibition of these transporters would lead to an increase in permeability / abundance / exposure of their substrates in plasma as well as brain/CSF (Figure 1-2, Figure 1-3). 


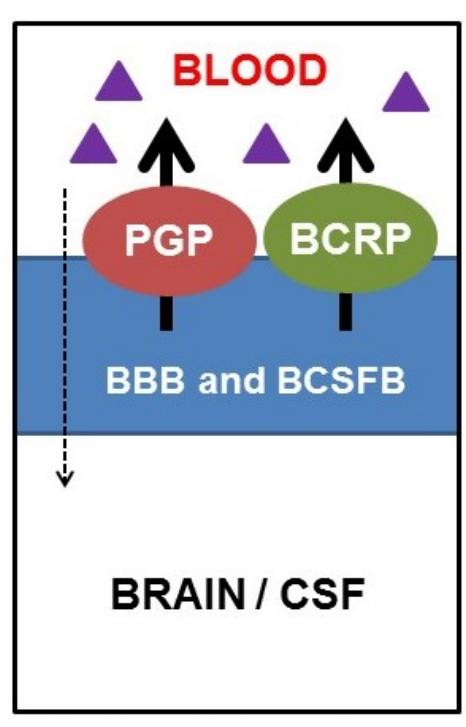

BBB - Blood Brain Barrier;

BCSFB - Blood CSF Barrier

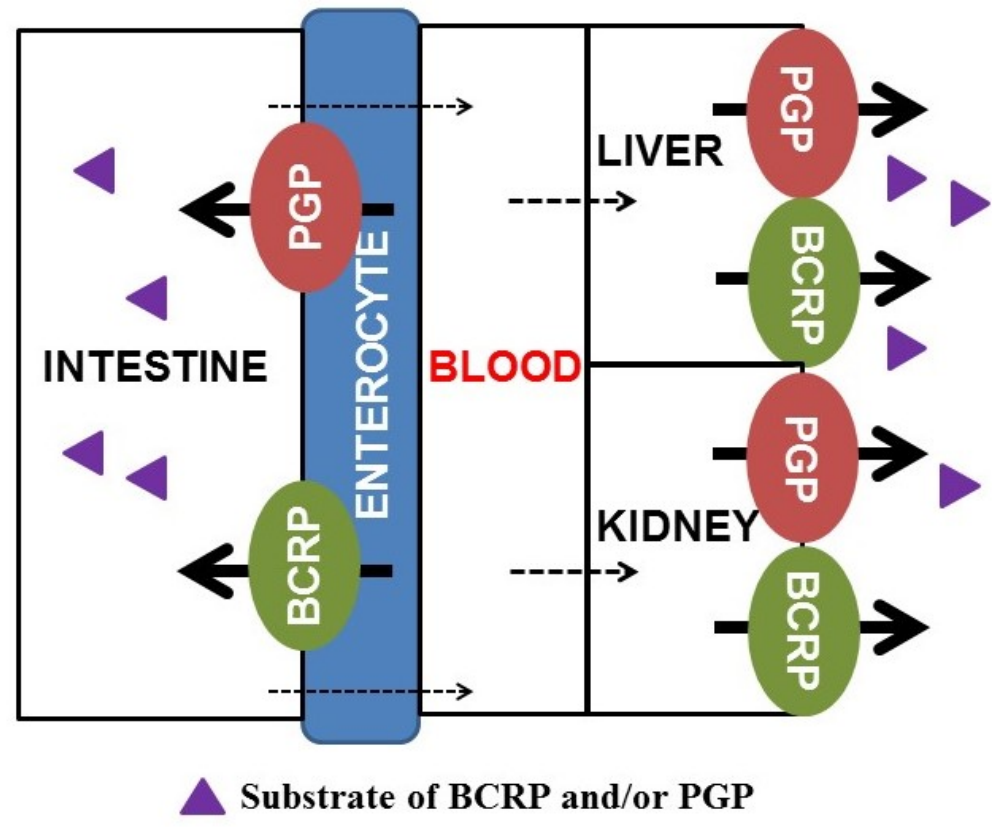

Substrate of BCRP and/or PGP

Figure 1-2. Effect of BCRP and PGP mediated efflux on brain/CSF and blood concentration of its substrates 


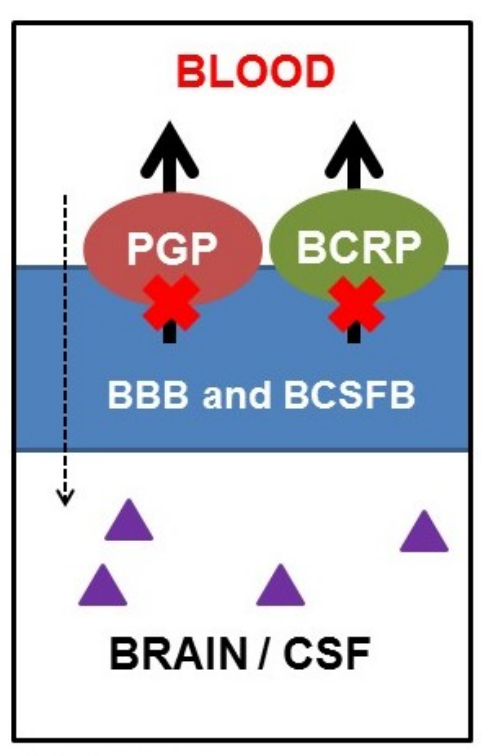

BBB - Blood Brain Barrier;

BCSFB - Blood CSF Barrier

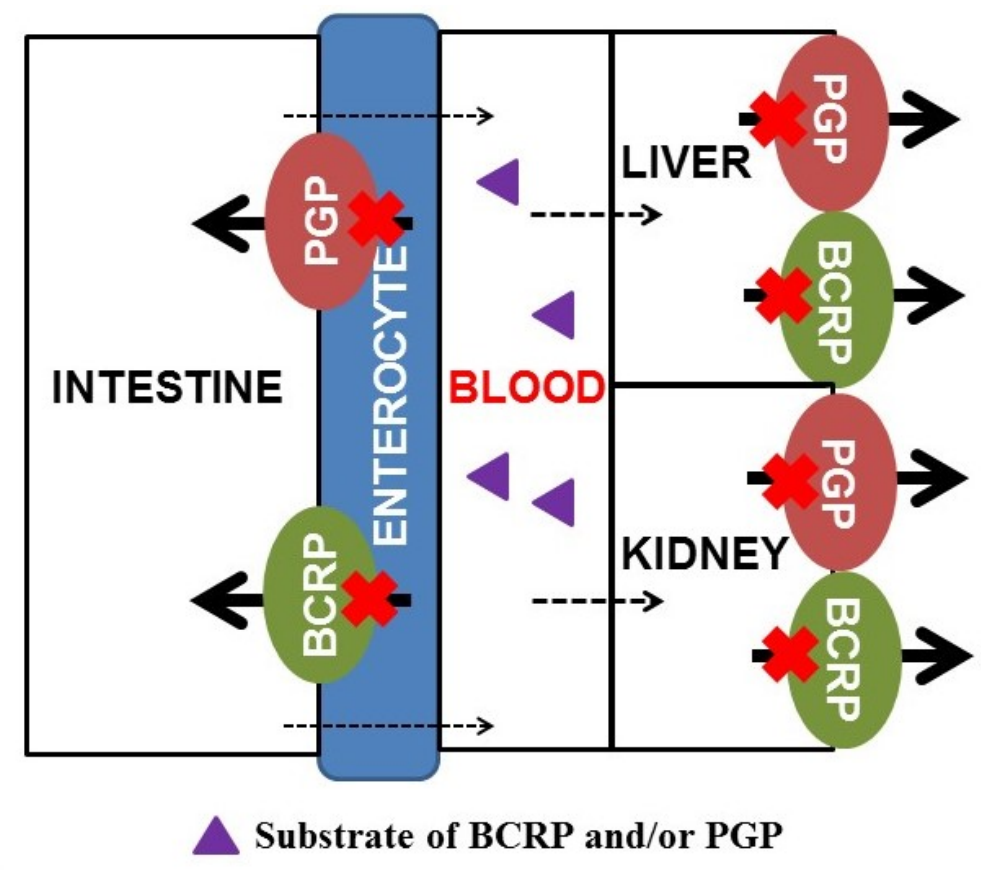

Figure 1-3. Effect of inhibition or absence of BCRP and PGP on the Brain/CSF and blood concentration of its substrates 


\section{Guidance about drug-drug interaction and substrate and/or inhibitor identification of BCRP and PGP}

ITC and FDA have provided important guidelines for identification of substrates and inhibitors of BCRP and PGP transporters [25] [1,80]. These two transporters are expected to have significant clinical drug-drug interaction (DDI) liabilities for orally administered drugs, due to their impact on oral absorption of their substrates [176] [177]. An efflux ratio of $\geq 2$ is recommended for a compound to be considered as a substrate, which should also be inhibitable by a known inhibitor at a concentration 10 times its coefficient of inhibition (Ki). For the selected highest clinical doses, dose/250 ml (Igut) should be 10-fold or greater than IC50 (concentration for 50\% inhibition) (Igut/IC50 $\geq$ $10)$ for considering an oral DDI, and maximum plasma concentration $\left(\mathrm{C}_{\max }\right)$ should be greater than 1-fold than IC50 for systemic interaction $\left(\mathrm{C}_{\max } / \mathrm{IC} 50 \geq 1.1\right)$ [71] [1]. In addition, concomitant medications that could be potential inhibitor(s) of BCRP/PGP need to be considered for making a clinically meaningful decision. For FDA recommended clinical substrate sulfasalazine, an AUC ratio change, in presence of the inhibitor, of $\geq$ 1.5-fold should be considered for DDI studies. Drug distribution into tissues where it can have potential safety concerns should also be considered for DDI studies.

\section{Metabolomics}

Metabolomics is the understanding of endogenous metabolites in a normal or disease condition, or after a drug treatment, or exposure to any chemical entities [178]. Metabolites are the terminal products of the principle of "Central Dogma" and could be useful by themselves, or in conjunction with proteomic and/or transcriptomic data for better understanding of biological processes and could be more closely related to a phenotype [179] [180]. While a focused study of metabolomic fingerprinting has been in use for a long time [181], metabolomics as a means of fingerprinting, phenotyping and understanding physiological functions and altered endogenous metabolites or biomarkers, has been recently employed as a remarkable tool [182] [183] [184]. Recently, metabolomics has been proposed to be used in "precision medicine" as a tool capable of defining the population variability in response to therapy [185]. Development of analytical tools such as LC-MS/MS, GC-MS and NMR or LC-NMR has created the opportunity for scientists to specifically detect multiple metabolites on a high throughput scale [186]. Different databases are used for analyzing the data obtained and develop meaningful pathway analysis of identified metabolites such as Kyoto Encyclopedia of Genes and Genomes (KEGG) and The Human Metabolome Database (HMDB) [187]. HMDB maintains a comprehensive list of metabolites identified in the human body, which have been detected and reported in various studies (http://www.hmdb.ca). Currently, there are 114,064 metabolite entries in this database, but only 18,602 metabolites have been detected and quantified, and 4,158 metabolites have only been detected. Majority of these metabolites are found in blood $(18,669)$, followed by feces (6738), urine (2407), saliva (1233) and few in CSF (450) (http://www.hmdb.ca/statistics). Of note, metabolites identified from animal studies may not often be found on either HMDB or KEGG databases. 
The metabolome comprises of all endogenous metabolites, those derived from microbiome and metabolites which are dietary or due to any medication. Study of metabolomics includes understanding changes in basal metabolites in response to changes in the environment, which includes disease, treatment, exercise and even alteration in the gut microbiome in response to the altered environment $[180,185]$.

\section{Untargeted and targeted metabolomics}

Study of metabolomics can be untargeted or targeted [179]. Untargeted metabolomics is, in theory, the study of the entire metabolome or metabolites identifiable within the limits of the protocol employed. Whereas it can be useful for novel understanding and identification of unknown pathways, or in cases lacking prior knowledge of the possible outcome, untargeted metabolomics generates a huge raw dataset that is difficult to analyze, hard to interpret and usually needs complex analytical procedures to render the results to a meaningful manageable dataset. Untargeted metabolomics is also not quantitative in nature.

In contrast, targeted metabolomics is more focused and may be semi-quantitative or quantitative depending on the project goals. Because it is more focused, targeted metabolomics involves determination of predefined metabolite changes or metabolic pathway changes and is easier to analyze and interpret. However, targeted metabolomics is limited by the amount of information that can be obtained, an example of which is the identification of dietary phytoestrogen glucuronide metabolites as MRP3 substrate [188].

\section{Analysis of untargeted metabolomics}

As discussed before, untargeted metabolomics requires extensive protocol development for the analytical procedure as well as identification of the metabolite signal with specificity and confidence [179]. For example, Metabolon ${ }^{\circledR}$, a company that performs metabolomic analysis for clients, maintains a library of carefully detected and identified metabolites by UPLC-MS, GC-MS based methods, with their spectral information, retention index(RI) and molecular weight/charge $(\mathrm{M} / \mathrm{Z})$ values for massspectrometry identified peaks [186].

For analysis of untargeted metabolomics data, a complex multivariate statistical approach similar to the analysis of genomic data is employed. Many online available tools such as MetaboAnalyst can be used for analysis [189]. Other methods such as metaXCMS [190], metal-server [191], InCroMap, MetacoreTM, 3Omics etc. also exist among other platforms [192]. 


\section{Integration of untargeted metabolomics and transcriptomics}

Integration of metabolomics with transcriptomic or gene expression change data can be a very useful method for complete understanding of perturbed or altered biological pathways [193]. Although some research articles using this approach have been published, integration of two very different datasets is quite complicated, and complex statistical approaches along with a carefully designed study is required for proper integration [194]. One major complexity is that change in one metabolite may be regulated by multiple genes, thereby rendering a simple association analysis useless. However, despite the complexity, integration of these two datasets is often attempted in literature [178] [195] [196].

\section{Use of metabolomics to understand endogenous function of transporters}

The physiological importance of drug transporters and their impact on the endogenous metabolome can be investigated using metabolomic analysis. Both targeted and untargeted metabolomic approaches have been reported, such as the use of untargeted metabolomics for identification of endogenous substrates or metabolites associated with OAT3 and OAT1 transporters in mice and targeted metabolomics to understand the effect of rosiglitazone treatment on the lipid metabolome [20, 140-142, 197].

Analysis of the metabolome may also lead to the identification of biomarkers or novel substrates of drug transporters, as shown by the identification of putative biomarker for OATP1B1 using metabolomics and genome-wide association study (GWAS) database [198].

The Metabolomic-GWAS server (http://metabolomics.helmholtzmuenchen.de/gwas/) needs a special mention in this regard, which comprises data from mostly two clinical GWAS-metabolomic analysis datasets [199, 200], that is extremely useful for identification of gene-metabolite association in humans.

\section{Study Purpose and Specific Aims}

BCRP and/or PGP transporters are known to transport xenobiotics as well as endogenous substrates. Lack of function polymorphisms of these transporters are also known to impact the disposition of their substrates in humans, as well as associated with pathological abnormalities. Although efforts are being taken to develop inhibitors of these transporters to overcome therapeutic resistance, studies are lacking towards a systemic understanding of the biological/endogenous role of these transporters. An understanding of the endogenous role of these transporters would be extremely helpful for identification of the effect of long-term inhibition of these transporters on the biological pathways. Therefore, the purpose of the current study was to elucidate the effect of Bcrp and/or Pgp transporters on the systemic metabolome and their 
physiological function in rats, towards developing a novel understanding of the effect of Bcrp and/or Pgp transporters on their endogenous, xenobiotic and dietary substrates.

\section{Specific aim 1}

The first aim of the current studies was to determine the role of Bcrp and Pgp efflux drug transporters on the systemic metabolome in rats. We hypothesized that untargeted metabolomics combined with transcriptomic analysis can help understand the endogenous function of these transporters.

\section{Specific aim 2}

The second aim of the current studies was to understand the effect of Bcrp and Pgp on ketamine, used as the animal anesthetic and identified as a putative dual substrate of these transporters in Aim 1 analysis. We hypothesized that Bcrp and/or Pgp can affect the pharmacokinetics (PK) and pharmacodynamics (PD) of ketamine in mice.

\section{Specific aim 3}

The third aim of the current studies was to utilize pheophorbide $\mathrm{A}(\mathrm{PhA}), \mathrm{a}$ dietary substrate of Bcrp, also identified in Aim 1 analysis, as an in vivo marker of Bcrp function. We hypothesized that PhA can be utilized for identification of oral drug-drug interactions due to Bcrp inhibition in mice. 


\section{CHAPTER 2. ELUCIDATION OF ENDOGENOUS FUNCTION OF BCRP AND PGP DRUG TRANSPORTERS}

\section{Introduction}

Drug transporters play an important physiological role by regulating the disposition of important endogenous metabolites. Efflux transporter multi-drug resistant protein 4 (Mrp4; Abcc4) is known to transport Prostaglandin E2 [60] and cAMP [201], while uptake transporters such as organic cation transporter (Octn2; Slc22a5) transports carnitine [202] and dopamine [203] and organic anion transporters (Oats) are known to transport uremic toxins [142]. P-glycoprotein (Pgp; Abcb1) and breast cancer resistant protein (Bcrp; Abcg2) are multi-drug efflux transporters that are ubiquitously present in different tissues and cell types in human and other mammals [46, 104]. The major function of Bcrp and Pgp is to control absorption of their substrates into blood from intestine, or increase their clearance at liver, kidney and other tissues, thereby regulating tissue exposure of their substrates. Thus, absence or inhibition of these transporters may lead to accumulation of their substrates, leading to toxicity or altered efficacy of their substrate drugs. These properties make Bcrp and Pgp among the most important FDAregulated efflux transporters for drug-approval processes [31].

Bcrp and Pgp are broad chemical specificity efflux transporters, with a substantial overlap in their substrates and inhibitors [102, 204] and a similar tissue distribution [205]. In addition, these transporters have been associated with the transport of important endogenous metabolites. For example, Bcrp is known to transport riboflavin (Vitamin B2) [9], uric acid [54] and folic acid [124], while Pgp-mediated transport has been reported for cortisol, aldosterone [57], phospholipids [161], bilirubin [160] and endogenous opioid peptides [58]. Both Bcrp and Pgp are well-conserved in the eukaryotic system and alteration in expression or association of these transporters has been reported in different disease conditions [54, 150, 173, 206, 207], implying their important physiological function. In addition, we have previously reported that apart from its expression on the apical surface of choroid plexus (CP) in mice [208], Bcrp is present on both apical and basolateral side at the arachnoid barrier (AB) epithelial cells, thus facing both blood and CSF [135]. Thus, role of Bcrp in CSF disposition of its substrates is complicated by the two opposing factors: (i) Bcrp reduces permeability of its substrates at BBB to brain and thus eventually in CSF and (ii) Bcrp increases efflux of its substrates from BCSFB into CSF. It also raises the possibility that Bcrp might have some important endogenous function at the BCSFB.

Metabolomic analysis is widely utilized to understand altered biological function in disease states [209], identification of biomarkers for disease [210] and drug treatment, [211] as well as for elucidation of endogenous functions or biomarkers for enzymes and transporters [198]. While untargeted metabolomics can lead to a broad systemic understanding, targeted metabolomics can answer questions related to specific metabolites in certain biological pathways [141, 179, 212]. Recently, untargeted metabolomics has been effectively utilized to identify endogenous substrates of OATP 
and OAT transporters $[140,142,198]$. An understanding of the transporter effect on metabolome can help elucidate pharmacometabolomics of drugs (that are transporter substrates or inhibitors), which might be useful to analyze pharmacokinetic variabilities, pharmacokinetic-pharmacodynamic relationships and toxicities or side effects associated with the drug treatment $[213,214]$. Several marketed drugs are known inhibitors of Bcrp and Pgp transporters, of which many are used for chronic therapy. Thus, a systems level understanding of the metabolomic impact and altered biological function thereof, due to lack of function of Bcrp and Pgp transporters, can lead to better understanding of the desired/ undesired effects of such therapy.

Therefore, to understand the effect of Bcrp/Abcg2 and Pgp/Abcb1a absence on the systemic metabolome, we performed an untargeted metabolomics study of plasma and CSF, as well as tissue transcriptomics of kidney, liver and brain (frontal cortex) from Bcrp-Pgp double knockout (dKO) and wild type (WT) rats. We chose to compare WT vs dKO male rat CSF and plasma because (a) BCRP shows significant sexual dimorphism (Male $>$ Female) in many tissues [215], hence male dKO would be expected to show the largest difference in substrate concentration; (b) CSF volume would be significantly higher in rats vs. mice; (c) dKO animals show a significantly higher concentration of dual Pgp/Bcrp substrates compared to single Pgp or Bcrp KO animals [94]; and (d) the $\mathrm{CSF} /$ brain concentration of Pgp or Bcrp KO mice is routinely different from WT while the plasma concentration does not always vary [144] hence sampling only the plasma would miss identifying some Pgp or Bcrp substrates.

Our results demonstrate a significant effect of Bcrp and Pgp transporters on plasma as well as CSF metabolome of rats, including a distinct impact in plasma vs. CSF compartment metabolomes. Utilizing the transcriptomic analysis and biological pathway information, our results also indicated important biological functions of Bcrp and Pgp efflux transporters. We also utilized an in silico Bcrp and Pgp-interaction prediction model to further identify metabolites that could be putative substrates of Bcrp and/or Pgp. To our knowledge, this is the first effort for complete systemic understanding of the endogenous biological function of efflux transporters.

\section{Materials and Methods}

\section{Chemicals and reagents}

Ketamine (JHP Pharmaceuticals, Rochester, MI, USA), Xylazine (LLOYD Laboratories, Shenandoah, Iowa, USA), scalp vein set (27G X 3/4" T.W.) for CSF collection (Exelint International,Co., LA, CA, USA), RNAlaterTM, TrizolTM and Affymetrix Rat 2.0 St Array (ThermoFisher Scientific, Little Rock, AR, USA) were purchased and used for the study. 


\section{Animal experiments and sample collection}

24 male wild-type (WT) and Bcrp - Pgp double knockout (dKO) Sprague-Dawley rats ( $\mathrm{n}=12$ each group), 10 weeks of age, obtained from SAGE Laboratories (Saint Louis, MO, USA), were used for the study. All animals were provided water and food ad libitum. All experimental procedures were approved by the Institutional Animal Care and Use Committee at St. Jude Children's Research Hospital.

Rats were anesthetized with a mixture of ketamine $(100 \mathrm{mg} / \mathrm{kg})$ and xylazine (10 $\mathrm{mg} / \mathrm{kg}$ ) and fixed on a stereotaxic apparatus. CSF was collected via the cisterna magna using a scalp vein set and a $100 \mu \mathrm{L}$ Hamilton syringe (Reno, Nevada, USA). CSF was immediately kept on dry ice, and later stored at $-80^{\circ} \mathrm{C}$. Immediately after CSF collection, blood was collected by cardiac puncture in EDTA-coated tubes, centrifuged at $5000 \mathrm{~g}$ for 5 minutes at $4{ }^{\circ} \mathrm{C}$, and plasma collected and stored at $-80^{\circ} \mathrm{C}$. Kidney, liver and brain frontal cortex were also isolated from each rat. The liver was frozen at $-80^{\circ} \mathrm{C}$ and brain frontal cortex and kidney were kept in RNAlaterTM following the manufacturer's instructions.

CSF and plasma samples were sent to Metabolon ${ }^{\circledR}$ for untargeted metabolomic analysis, and kidney, liver and brain frontal cortex tissues were utilized for transcriptomics analysis. A brief outline of the study design is illustrated in Figure 2-1.

\section{Metabolomic analysis}

Untargeted metabolomics of CSF and plasma samples from WT and dKO rats ( $\mathrm{n}=8$ each genotype) was performed by UPLC-MS/MS and GC-MS based methods at Metabolon ${ }^{\circledR}\left(\right.$ Durham, NC, USA) [186]. Metabolon ${ }^{\circledR}$ maintains a metabolite library consisting of retention index $(\mathrm{RI})$, mass/charge $(\mathrm{m} / \mathrm{z})$ and chromatographic data of authenticated standard metabolites including the MS/MS spectral information. Named metabolites in the sample were identified by comparing the mass ( $\pm 0.005 \mathrm{amu}), \mathrm{RI}$ and spectral data of the sample peaks with the library standards, the area under the peak quantified, median normalized and then compared between the WT and dKO groups using the two-sample t-test with Welch's correction. An estimate of false discovery rate (q-value) was also calculated because of multiple comparisons and $\mathrm{q}<0.1$ was considered to have higher confidence in the data. Significant metabolites were identified based on $p<0.05$ and highlighted dark red for increase or dark green for decrease in CSF of $\mathrm{dKO}$ compared to WT rats. Trending significant is considered when $0.1>\mathrm{p}>0.05$ and highlighted light red for increase or light green for decrease in CSF and plasma of dKO compared to WT rats. Principal component analysis was used to identify the data pattern [216] in the samples (WT vs dKO plasma and WT vs dKO CSF). Results were further analyzed by Random Forest Analysis to identify important metabolic features that contribute the most towards the group separation. The final output of fold change and associated $p$ and $q$ values were log2-transformed and volcano plots created on Tibco ${ }^{\circledR}$ Spotfire ${ }^{\circledR} 7.5$ for better visualization and identification of the significant metabolites. Based on the hierarchical clustering analysis (heatmap) by Metabolon ${ }^{\circledR}$, three CSF 


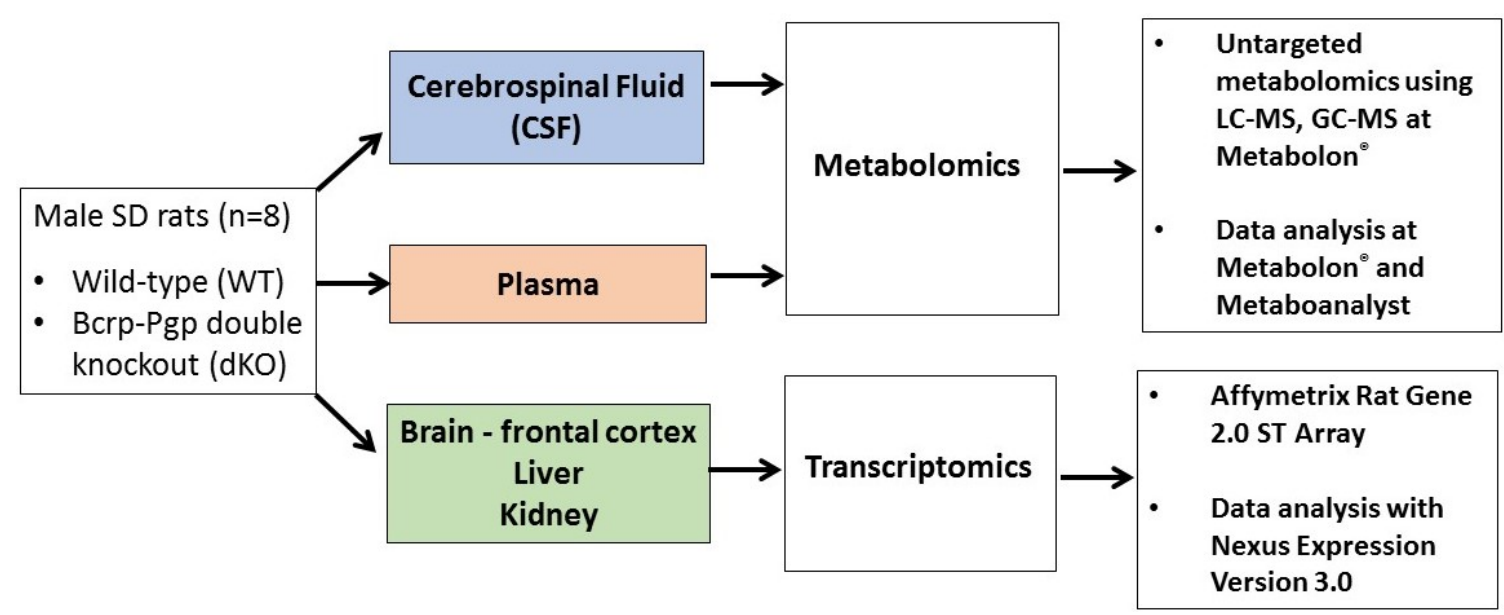

Figure 2-1. Outline of the study design 
samples were assumed to be contaminated with plasma and this information was used to calculate 'no contamination' fold change and p-values for the CSF samples, and is listed along with the uncorrected result for all CSF metabolite results generated by Metabolon $\AA$. The non-normalized raw data from Metabolon ${ }^{\circledR}$ was used to calculate the ratio of the signal intensity of metabolites that were detected both in CSF and plasma (CSF/plasma ratio). CSF/plasma ratio of the metabolites were analyzed by two sample ttest considering unequal variance to identify metabolites that have significant alterations in CSF abundance in the dKO rats compared to WT rats. Fold change of (CSF/plasma) $\mathrm{dKO} /(\mathrm{CSF} /$ plasma) _ WT were $\log 2$-transformed and plotted against $\log 2$ (t values) on Tibco® Spotfire ${ }^{\circledR} 7.5$ for further identification and better visualization of metabolites with significantly altered CSF abundance in the dKO rats.

\section{Metabolomic analysis using MetaboAnalyst 3.0}

Raw metabolite intensity data from Metabolon ${ }^{\circledR}$ was further analyzed separately using the MetaboAnalyst software. Briefly, raw data was first screened for missing values and any metabolite with more than $50 \%$ missing values was omitted from further analysis. Due to a low number of features $(<2000)$, the data filtration step was skipped. Data was further median-normalized and log-transformed to obtain a Gaussian data distribution for further statistical analysis. Significant metabolites were identified based on $\geq 1.5$ - fold increase or decrease in median intensity in dKO compared to WT CSF or plasma, and a p-value less than 0.05. Partial least square discriminant analysis and hierarchical clustering analysis was performed to assess the group separation (WT vs. $\mathrm{dKO})$. The list of significant metabolites was compared with the Metabolon ${ }^{\circledR}$ data to assess the reproducibility of the significant features.

\section{RNA extraction and microarray analysis}

Brain, kidney and liver tissues were weighed, diluted 10 times with TriazolTM, homogenized and RNA isolated according to the manufacturer's instructions. RNA samples were analyzed for quality and integrity on the Agilent 2100 Bioanalyzer and the concentration of RNA was determined using NanoDropTM 8000 spectrophotometer. A hundred nanograms (100 ng) of total RNA was processed for use on the microarray using the Ambion WT kit and the Affymetrix Terminal labeling kit according to the manufacturer's instructions. The resultant single-stranded cDNA was labeled, fragmented and then hybridized to the Rat Gene 2.0 ST array for sixteen hours at 45 at $60 \mathrm{rpm}$. The arrays were then washed, stained, and scanned using the Affymetrix Model 450 Fluidics Station and Affymetrix Model 3000 7G scanner using the manufacturer's recommended protocols.

Affymetrix Rat 2.0 ST array was used to perform the gene expression level analysis using Affymetrix Expression Console software and results saved as RMA (Robust Multiarray Averaging) level signal. The RMA signal file was uploaded into Nexus expression 3.0 software (BioDiscovery Inc., El Segundo, CA, USA) along with 
the biological pathway information for the Affymetrix Rat Gene 2.0 ST Array, pooled based on intensity, corrected for multiple test comparisons at $\mathrm{q}<0.05$ and fold change $\geq 1.2$, to identify the significantly altered genes and biological pathways.

\section{In silico prediction of BCRP and/or PGP putative substrates or inhibitors}

Metabolites identified by the Metabolon ${ }^{\circledR}$ as significant $(\mathrm{p}<0.05)$ or trending significant $(0.05<\mathrm{p}<0.1)$ were analyzed for their interaction with BCRP and/or PGP by a predictive Bayesian Machine learning model implemented in Assay Central at Collaborations Pharmaceuticals according to the previously published methods [217, 218]. Models were generated for human PGP using 1217 molecules and human BCRP with 392 molecules. A five-fold cross-validation was performed to assess the receiver operator characteristic (ROC). The metabolites were then scored with these models to output a score. Further, physicochemical descriptors, e.g. AlogP, molecular polar surface area, hydrogen bond acceptor and donor counts, molecular weight, for the metabolites were obtained from the public database. Regression analysis employing standard least square method was used to predict observed fold changes (dKO/WT) in the plasma and CSF for each metabolite using the molecular descriptors, along with the ABCG2 or PGP Bayesian model score to determine a structure-property relationship and understand if the metabolites detected in CSF and plasma have any predictive property of BCRP and / or PGP substrate.

\section{Results}

\section{Bcrp and Pgp gene deletion in rats results in altered metabolome of CSF and plasma} analysis:

The following four sections describe the overall findings of the metabolomic

1. Metabolomics analysis identified differentially expressed metabolites between $W T$ and $d K O$ rats, in both CSF and plasma. To identify the effect of Bcrp and Pgp gene deletion on metabolite expression, we performed untargeted metabolomics analysis in CSF and plasma of WT and Bcrp-Pgp dKO rats. A total of 564 named metabolites were identified in both WT and dKO rats, with 306 metabolites uniquely identified in plasma, 25 metabolites identified only in CSF and 233 metabolites identified in both CSF and plasma (Figure 2-2A). Data analysis for significant metabolite changes was performed independently at Metabolon ${ }^{\circledR}$ and in-house using the MetaboAnalyst software 3.0. Principal component analysis (PCA) (Figure 2-3A and B) and Partial Least Square Discriminant Analysis (PLS-DA) (Figure 2-2B and C) identified distinctly different patterns for the metabolites expressed by WT and $\mathrm{dKO}$ rats in both CSF and plasma. In addition, hierarchical clustering analysis performed at Metabolon ${ }^{\circledR}$ (Figure 2-3C and D), and on MetaboAnalyst (Figure 2-2D and $\mathbf{E}$ ) demonstrated that majority of the samples clustered within their own group (WT or dKO) in both CSF and plasma. 
A

Total metabolites distribution

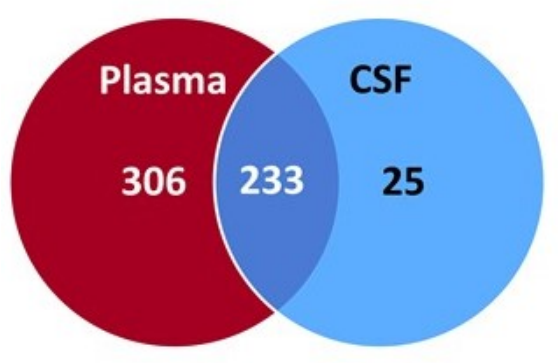

B

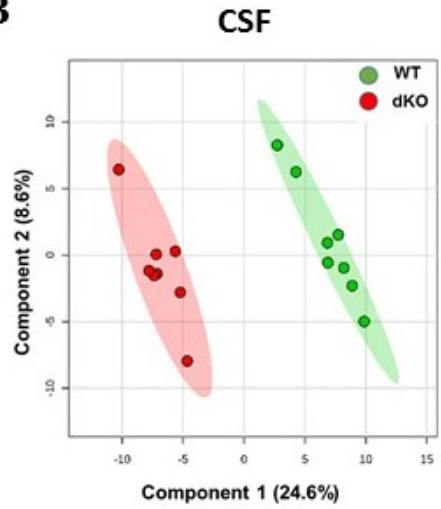

C Plasma

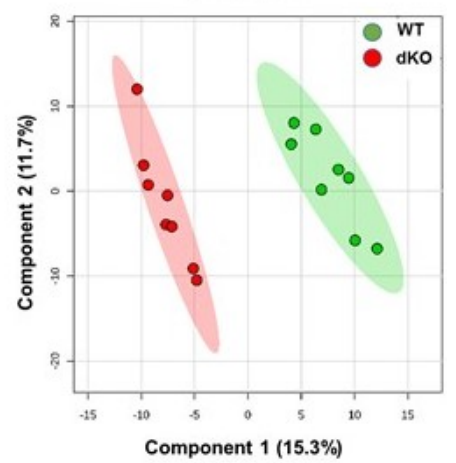

D

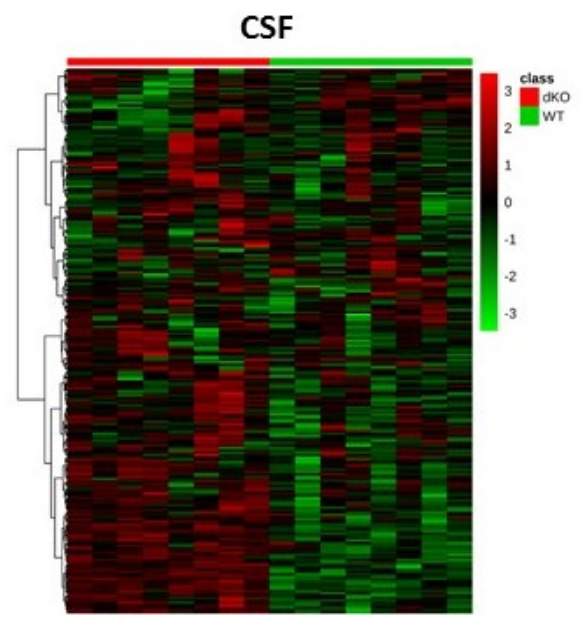

$\mathbf{E}$

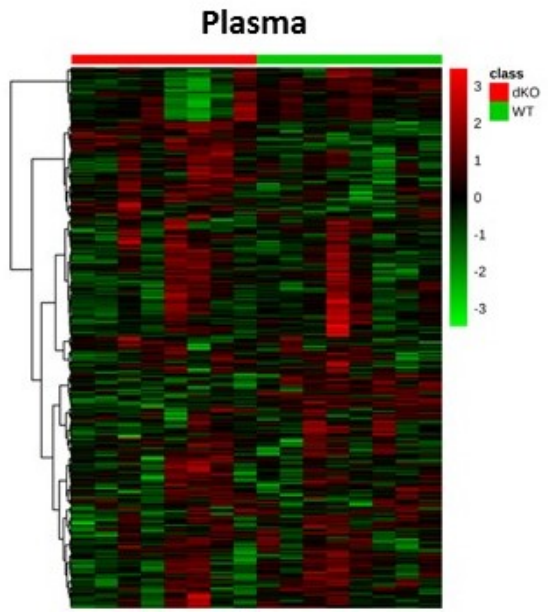

Figure 2-2. Metabolites in CSF and plasma are differentially expressed between WT and Bcrp-Pgp dKO rats (MetaboAnalyst)

Notes: Venn-diagram showing number distribution of detected named metabolites in CSF and plasma samples (A). PLS-DA analysis 2D-scores plot performed on MetaboAnalyst 3.0, showing clear group separation between WT and dKO in CSF (B) and plasma (C). Hierarchical clustering of the normalized metabolomic data clusters each of WT and dKO groups together based on their metabolite features in CSF (D) and plasma (E). 
A

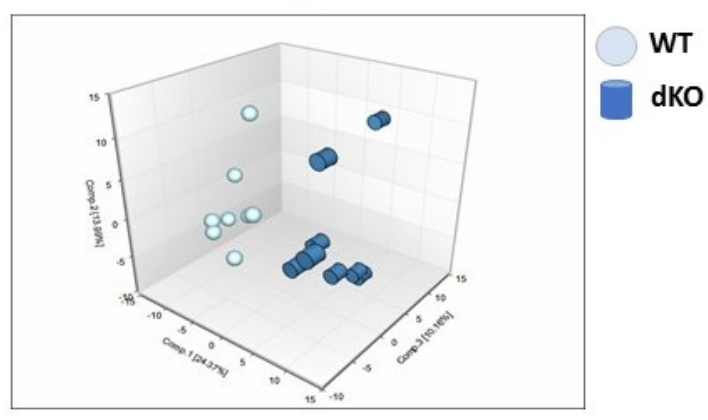

C

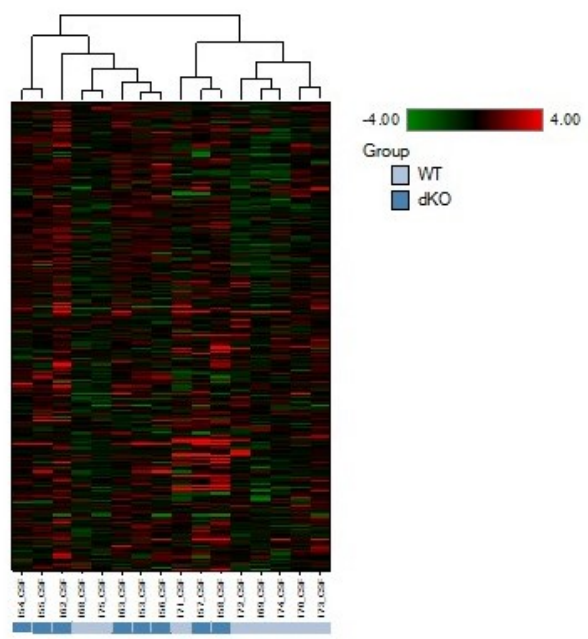

B

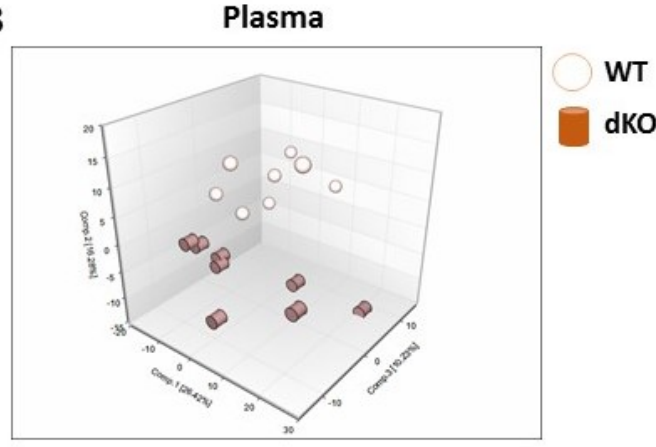

D

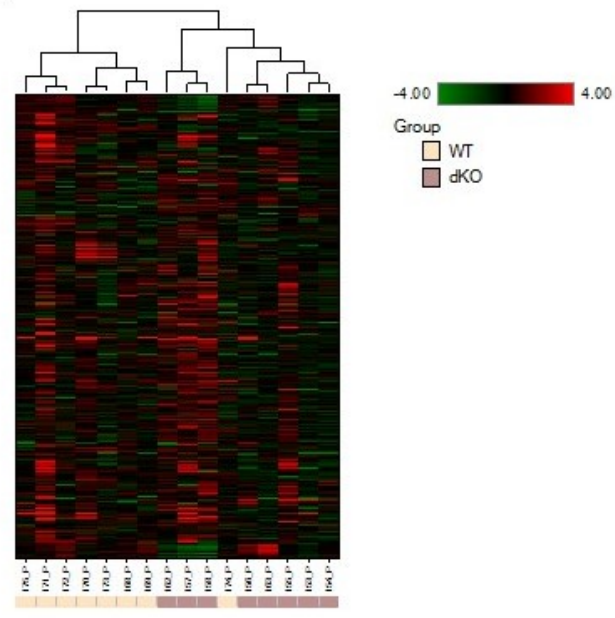

Figure 2-3. Metabolites in CSF and plasma are differentially expressed between WT and Bcrp-Pgp dKO rats (Metabolon ${ }^{\circledR}$ )

Notes: Principal Component Analysis of median normalized named metabolites in CSF (A) and plasma (B) identified significant pattern separation between WT and dKO rats. Hierarchical clustering analysis of the fold change (dKO/WT) data in CSF $(\mathbf{C})$ and plasma (D) showed overall separation between WT and dKO rats. 
2. Significantly altered metabolites in CSF and plasma of dKO rats (compared to WT) displayed unique signatures. A total of 169 metabolites were found to be significantly altered together in the CSF and plasma of dKO rats compared to WT rats. Of these, 79 metabolites were significantly altered only in the plasma, 71 only in the CSF and 19 metabolites were altered in both CSF and plasma of dKO rats, compared to WT rats (Figure 2-4A). The features of the metabolites altered in CSF and plasma were found to be strikingly different. While majority of the metabolites altered in CSF were amino acid-related metabolites (57\%) (Figure 2-4B), lipids comprised the major metabolites (49\%) significantly altered in the plasma of dKO rats, compared to WT rats (Figure 2-4C).

Volcano plots $(\log 10(q)$ vs $\log 2($ fold change) $)$ of the metabolome data revealed those metabolites that were significantly increased vs. decreased (Figure 2-5 and Figure 2-6). In addition, we found that most of the metabolites in CSF were increased in dKO rats, compared to WT (Figure 2-5), while an equal number of metabolites were increased as were decreased in the dKO plasma (Figure 2-6). The metabolites with the highest fold-change increases in dKO rats were O-methyl catechol sulfate in CSF (5.5fold) and 4-hydroxychlorothalonil (3.6-fold) in plasma. Only one metabolite, indolin-2one, was found to be significantly decreased ( 0.29 -fold) in the CSF of dKO rats while many lipid metabolites were significantly decreased in dKO plasma.

Random Forest Analysis (RFA) was performed to identify the metabolites with the greatest effect on group separation (WT and dKO) in CSF and plasma. RFA identified group separation with $100 \%$ prediction accuracy and results were plotted based on effect of each metabolite on mean decrease accuracy, displaying 30 metabolites with increasing order of their importance to group separation from bottom to top (Figure 2-7 and Figure 2-8). Among the metabolites with a significant effect on group separation, RFA identified known Bcrp substrates riboflavin and urate in the CSF metabolome and riboflavin and pheophorbide $\mathrm{A}$ in the plasma metabolome. RFA also confirmed our previous results that CSF and plasma maintain unique metabolomic signatures with significant metabolites being related to amino acids in the CSF and lipids in the plasma.

Analysis of the data with MetaboAnalyst using a more stringent data filtering protocol identified 29 metabolites altered significantly in the CSF (Figure 2-9) and 47 metabolites in the plasma (fold change $\geq 1.5$ ) (Figure 2-10) of dKO rats compared to WT rats, with metabolites listed in increasing order of significance from top to bottom. A list of metabolites which were significantly altered in CSF (Supplement_Chapter 2_Metabolites.Table S1A) and plasma (Supplement_Chapter 2_Metabolites Table S1B) of dKO vs WT rats when analyzed by both Metabolon ${ }^{\circledR}$ and MetaboAnalyst is provided, identifying a common list of altered metabolites, thereby incorporating robustness in our data analysis.

As expected, known endogenous and dietary substrates of Bcrp were increased in both CSF and plasma of dKO rats, compared to WT rats (Figure 2-11). While riboflavin, a known Bcrp substrate, was found to be increased in both CSF and plasma of dKO rats, urate was found to be significantly higher in abundance only in the CSF of dKO rats. 
A

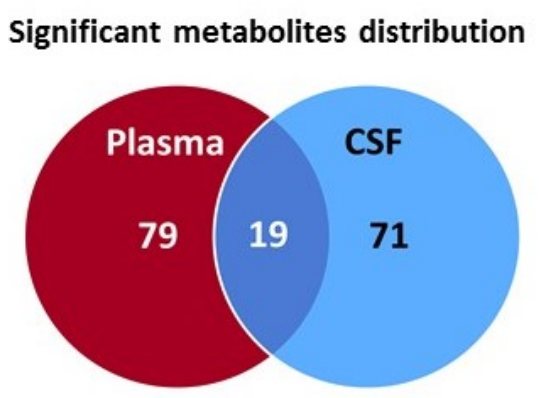

B

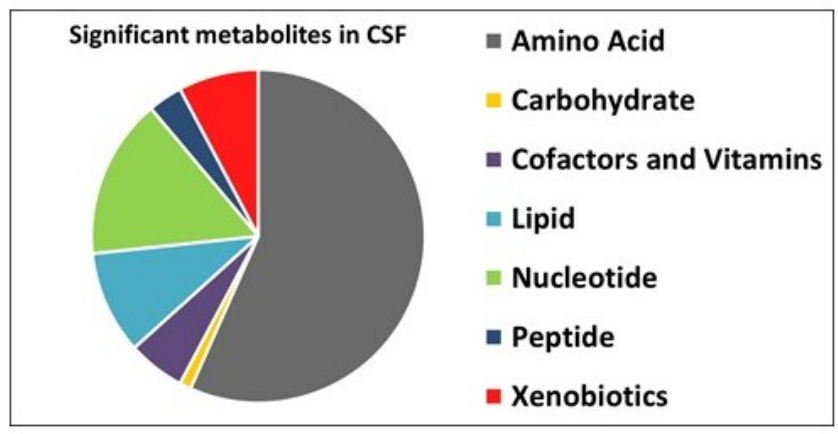

C

\begin{tabular}{|c|c|}
\hline Significant metabolites in Plasma & $\begin{array}{l}\text { " Amino Acid } \\
=\text { Carbohydrate } \\
\text { " Cofactors and Vitamins } \\
\text { " Lipid } \\
\text { = Nucleotide } \\
\text { " Peptide } \\
\text { " Xenobiotics }\end{array}$ \\
\hline
\end{tabular}

Figure 2-4. Significantly altered metabolites in CSF and plasma of dKO rats (compared to WT) display unique class signatures

Notes: Venn-diagram showing distribution of significant $(\mathrm{p}<0.05)$ metabolites in CSF and plasma of dKO vs. WT rats (A). Pi-chart showing distribution of percentage of significant $(\mathrm{p}<0.05)$ metabolites in different metabolite classes as designated by the colors in CSF (B) and plasma (C) of dKO vs. WT rats. 


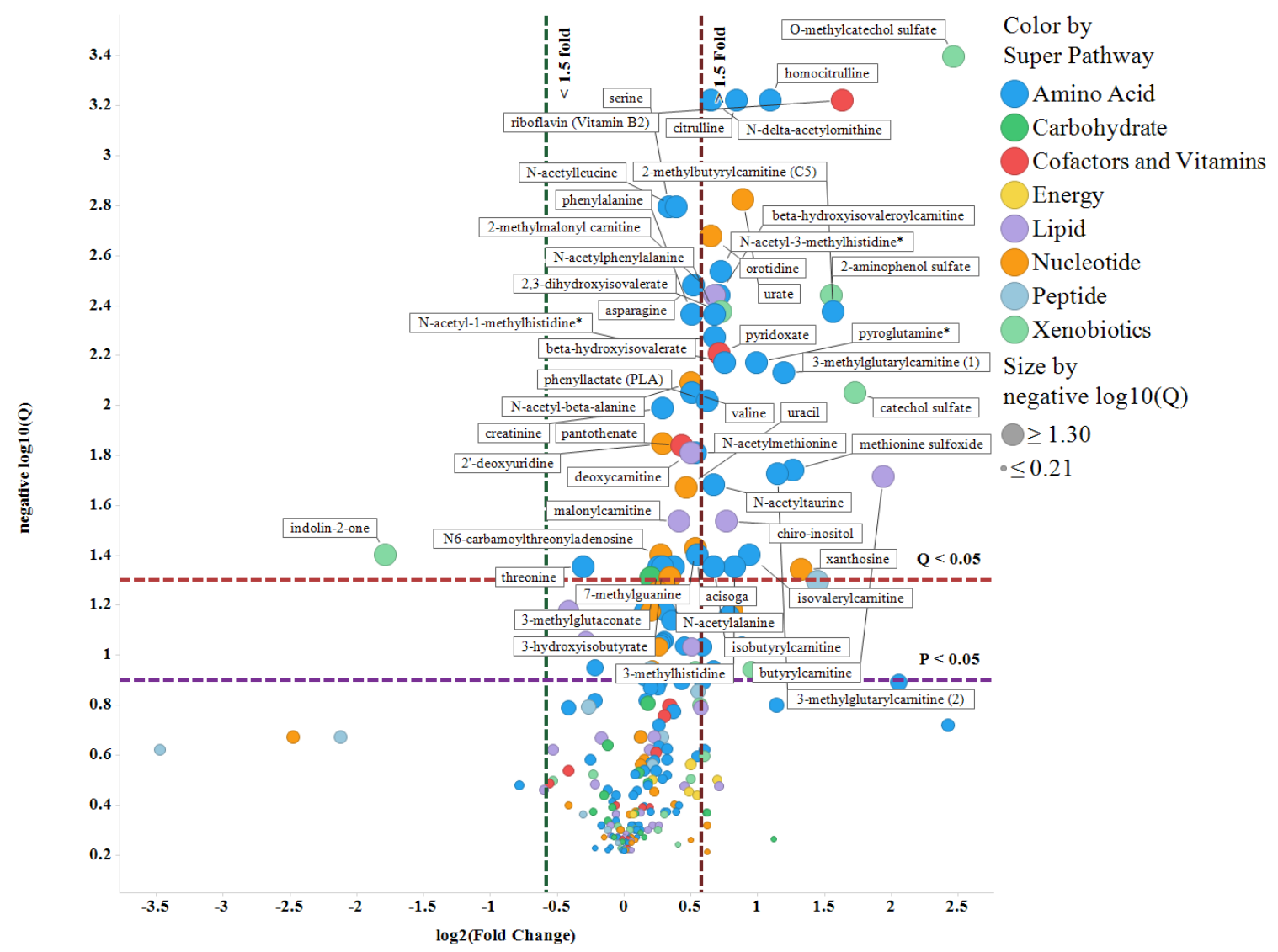

Figure 2-5. Significantly altered metabolites in CSF of dKO rats (compared to WT) display unique class signatures: Volcano plot in CSF

Notes: Volcano plot of CSF metabolites identified by pathway information (color of markers) and their significance (p-value) (size of marker). Data is plotted as log2 (dKO/WT metabolite raw signal intensity) on X-axis vs. negative log (Q-value of metabolite comparison dKO vs WT) on Y-axis. Horizontal purple and red lines designate $\mathrm{p}<0.05$ and $\mathrm{q}<0.05$ respectively. Significant metabolites $(\mathrm{p}<0.05)$ are identified above the purple line. Similarly metabolites with 1.5 -fold increase or decrease are identified with vertical red and green lines respectively. Metabolites on the right side of red line or on the left side of green line and above the horizontal purple line are significantly increased $(>1.5$ fold) or decreased $(<1.5$ fold) in CSF. 


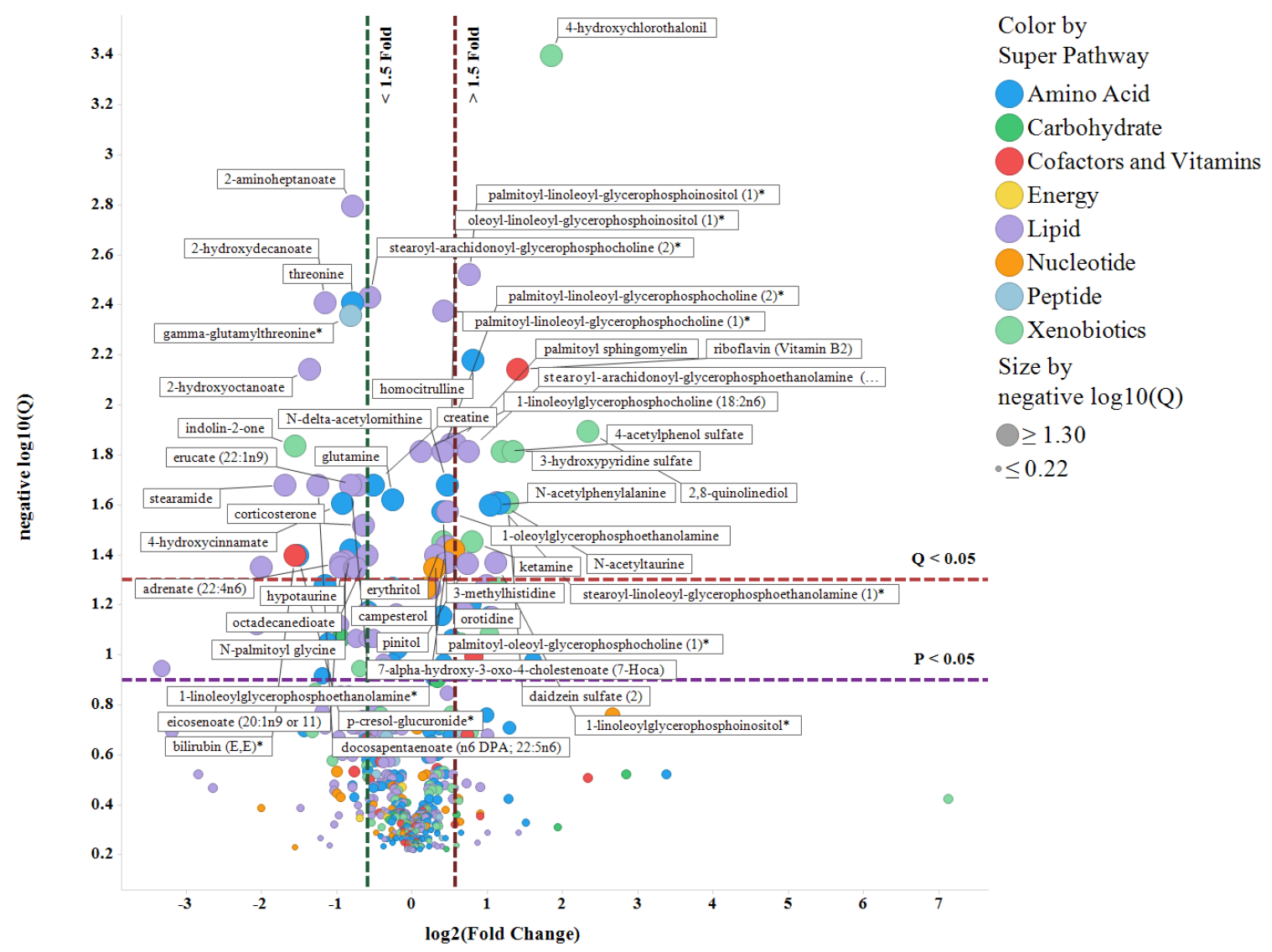

Figure 2-6. Significantly altered metabolites in plasma of dKO rats (compared to WT) display unique class signatures: Volcano plot in plasma

Notes: Volcano plot of plasma metabolites identified by pathway information (color of markers) and their significance (p-value) (size of marker). Data is plotted as log2 (dKO/WT metabolite raw signal intensity) on X-axis vs. negative log (Q-value of metabolite comparison $\mathrm{dKO}$ vs WT) on Y-axis. Horizontal purple and red lines designate $\mathrm{p}<0.05$ and $\mathrm{q}<0.05$ respectively. Significant metabolites $(\mathrm{p}<0.05)$ are identified above the purple line. Similarly, metabolites with 1.5 -fold increase or decrease are identified with vertical red and green lines respectively. Metabolites on the right side of red line or on the left side of green line and above the horizontal purple line are significantly increased $(>1.5$ fold $)$ or decreased $(<1.5$ fold $)$ in plasma. 


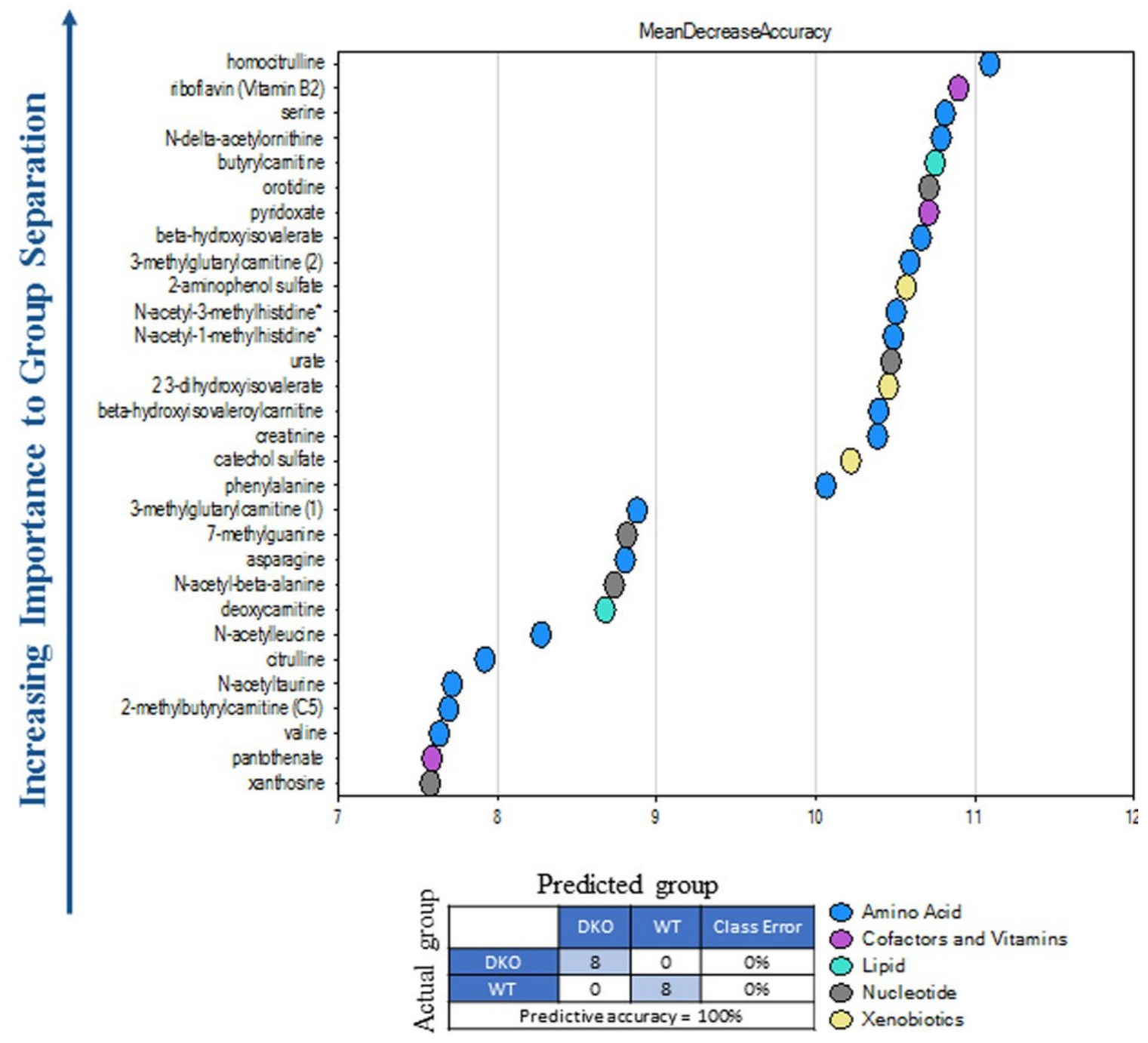

Figure 2-7. Random forest analysis identifies metabolites that significantly distinguishes between WT and dKO group of rats in CSF

Notes: Random forest analysis (RFA) was used to calculate group separation and identify metabolites with highest importance for group separation. RFA identified two groups (WT and dKO) with 100\% accuracy in CSF. Metabolites were plotted against their Mean Decrease in Accuracy, calculated by RFA. Metabolites have higher importance on group separation as we move up along the $\mathrm{Y}$-axis and they are also color coded to identify which pathway the metabolites belong to. 


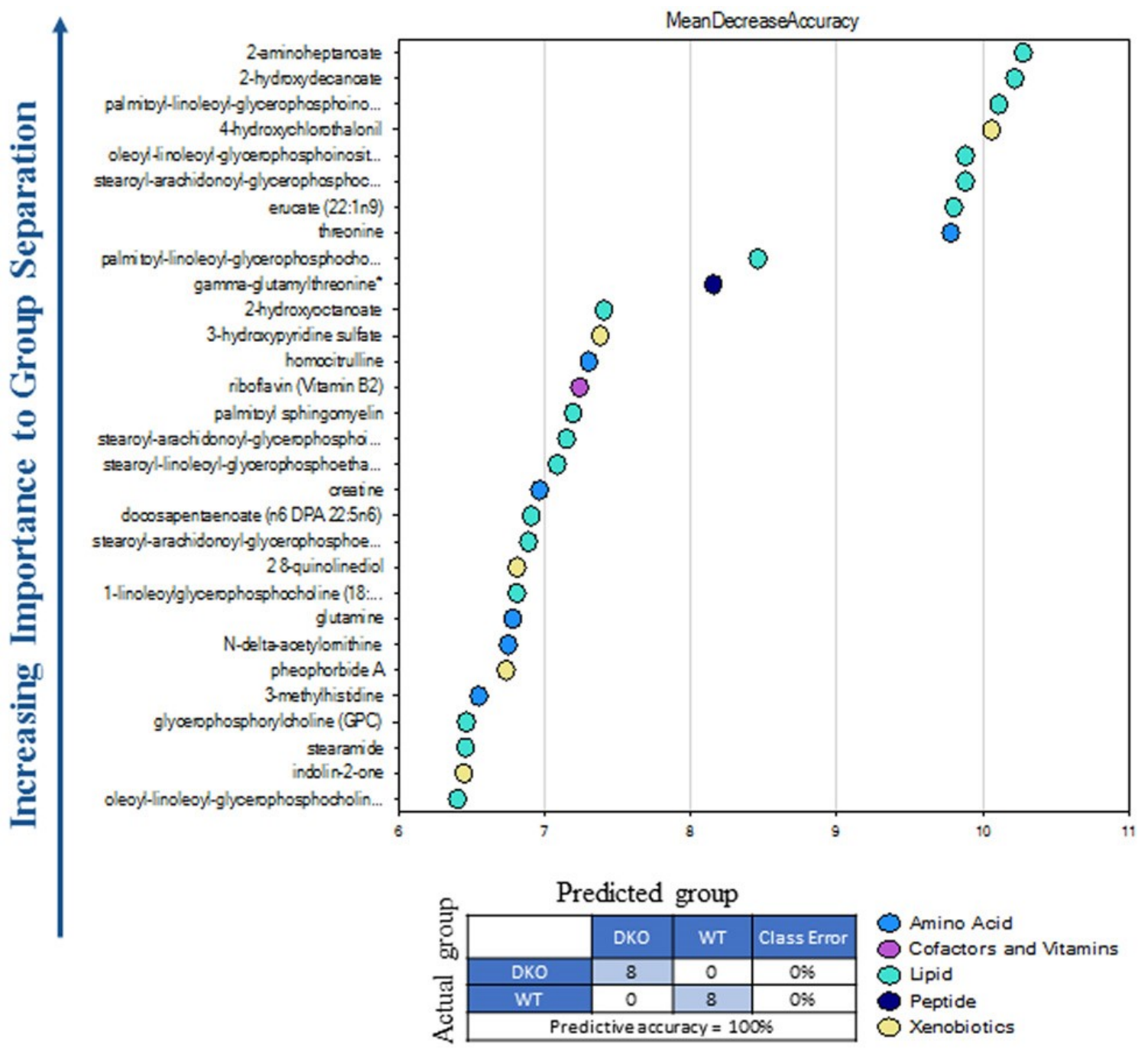

Figure 2-8. Random forest analysis identifies metabolites that significantly distinguishes between WT and dKO group of rats in plasma

Notes: Random forest analysis (RFA) was used to calculate group separation and identify metabolites with highest importance for group separation. RFA identified two groups (WT and dKO) with 100\% accuracy in plasma. Metabolites were plotted against their Mean Decrease in Accuracy, calculated by RFA. Metabolites have higher importance on group separation as we move up along the $\mathrm{Y}$-axis and they are also color coded to identify which pathway the metabolites belong to. 


\section{Significant metabolites in CSF: $p<0.05$ and Fold change $>1.5$}

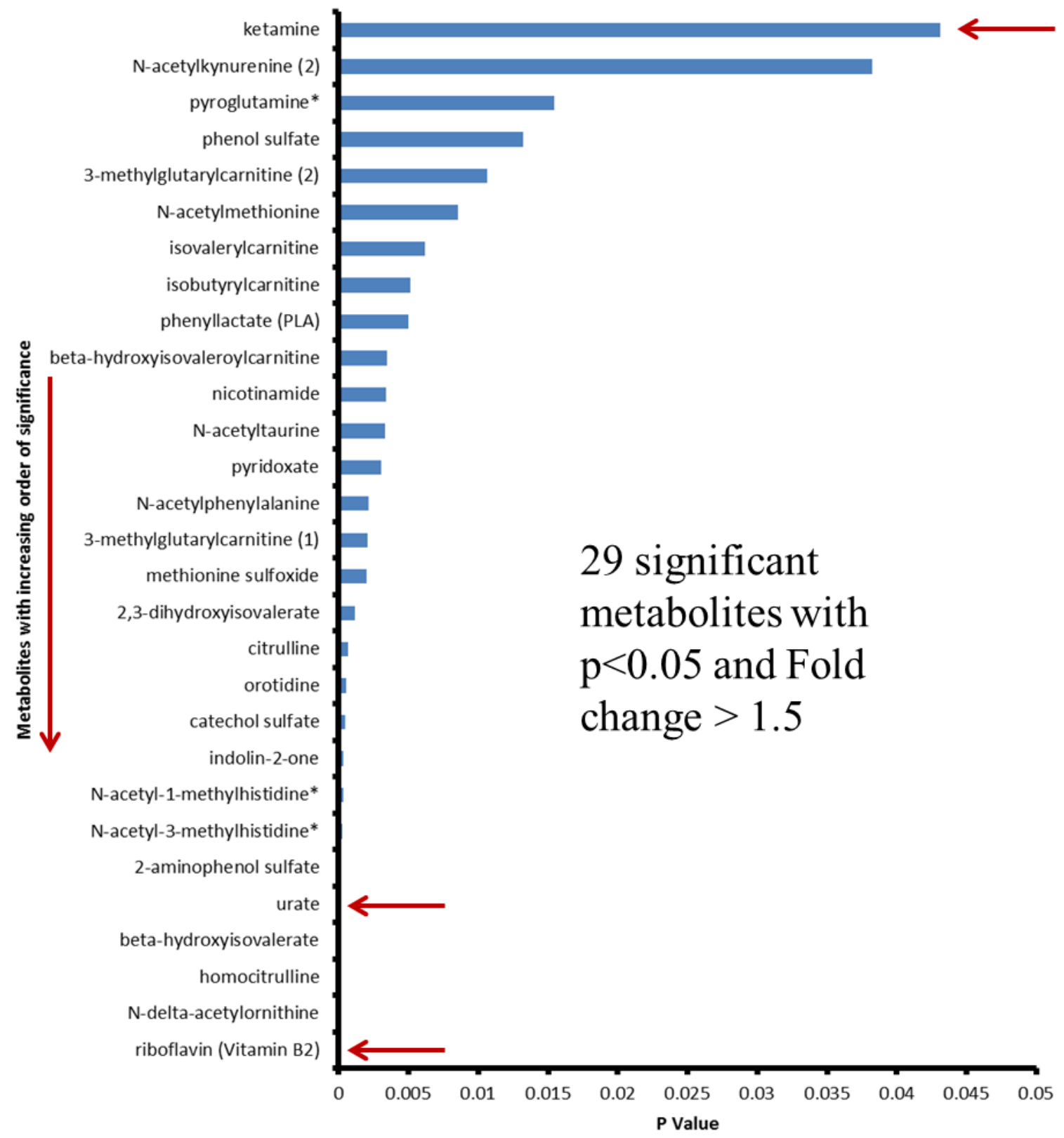

Figure 2-9. Independent analysis of metabolome data with MetaboAnalyst identifies similar metabolites, along with known Bcrp substrates in CSF

Note: Raw data from Metabolon were reanalyzed using MetaboAnalyst 3.0. Significant metabolites were identified based on $p<0.05$ and fold change $>1.5$. Fold change of all significant metabolites were plotted against their $\mathrm{p}$-value. The red arrow indicates known substrates of Bcrp and/or Pgp. 


\section{Significant metabolites in Plasma: $p<0.05$; Fold change $>1.5$}

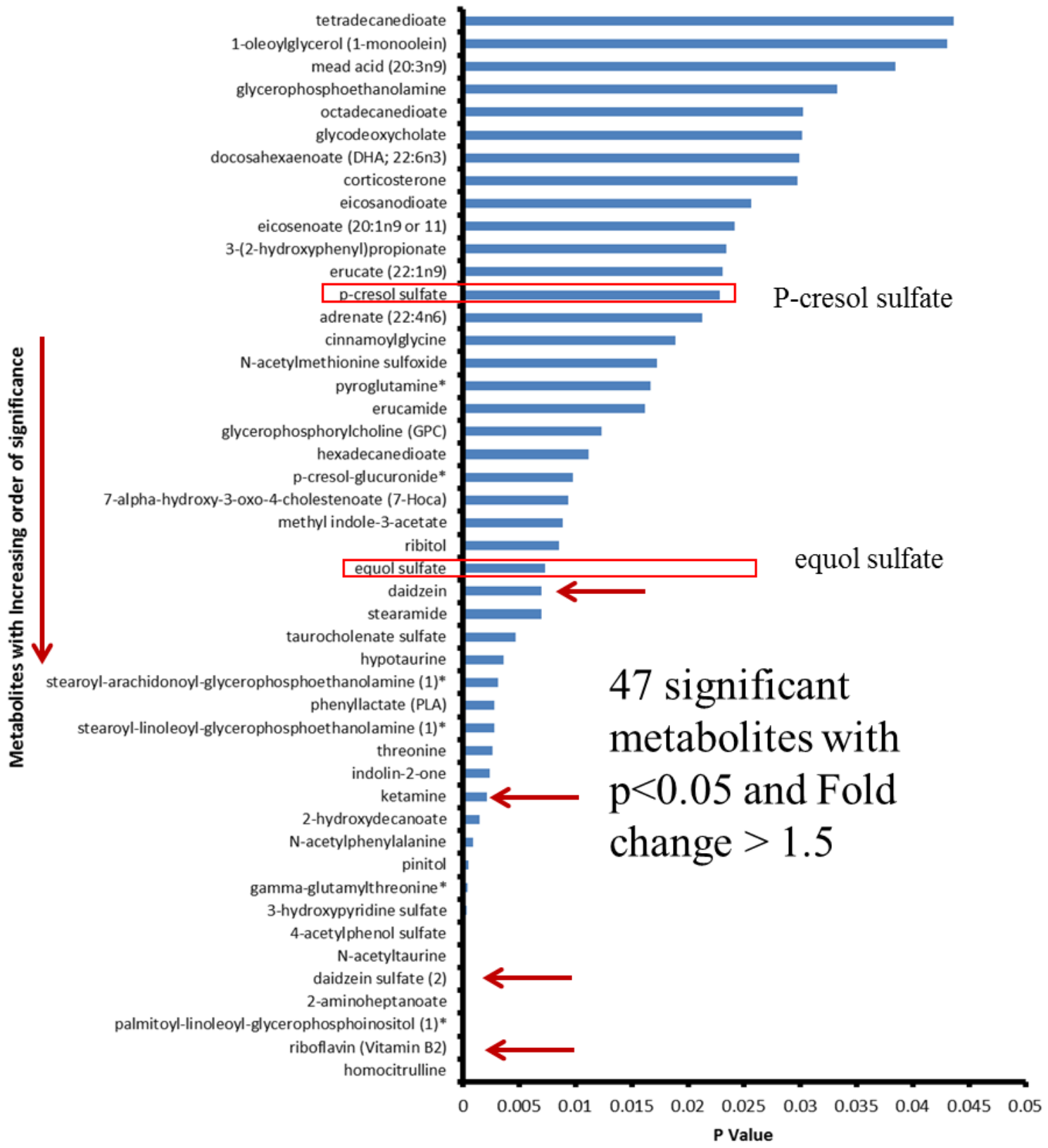

Figure 2-10. Independent analysis of metabolome data with MetaboAnalyst identifies similar metabolites, along with known Bcrp substrates in plasma

Notes: Raw data from Metabolon were reanalyzed using MetaboAnalyst 3.0. Significant metabolites were identified based on $p<0.05$ and fold change $>1.5$. Fold change of all significant metabolites were plotted against their $\mathrm{p}$-value. The red arrow indicates known substrates of Bcrp and/or Pgp. 

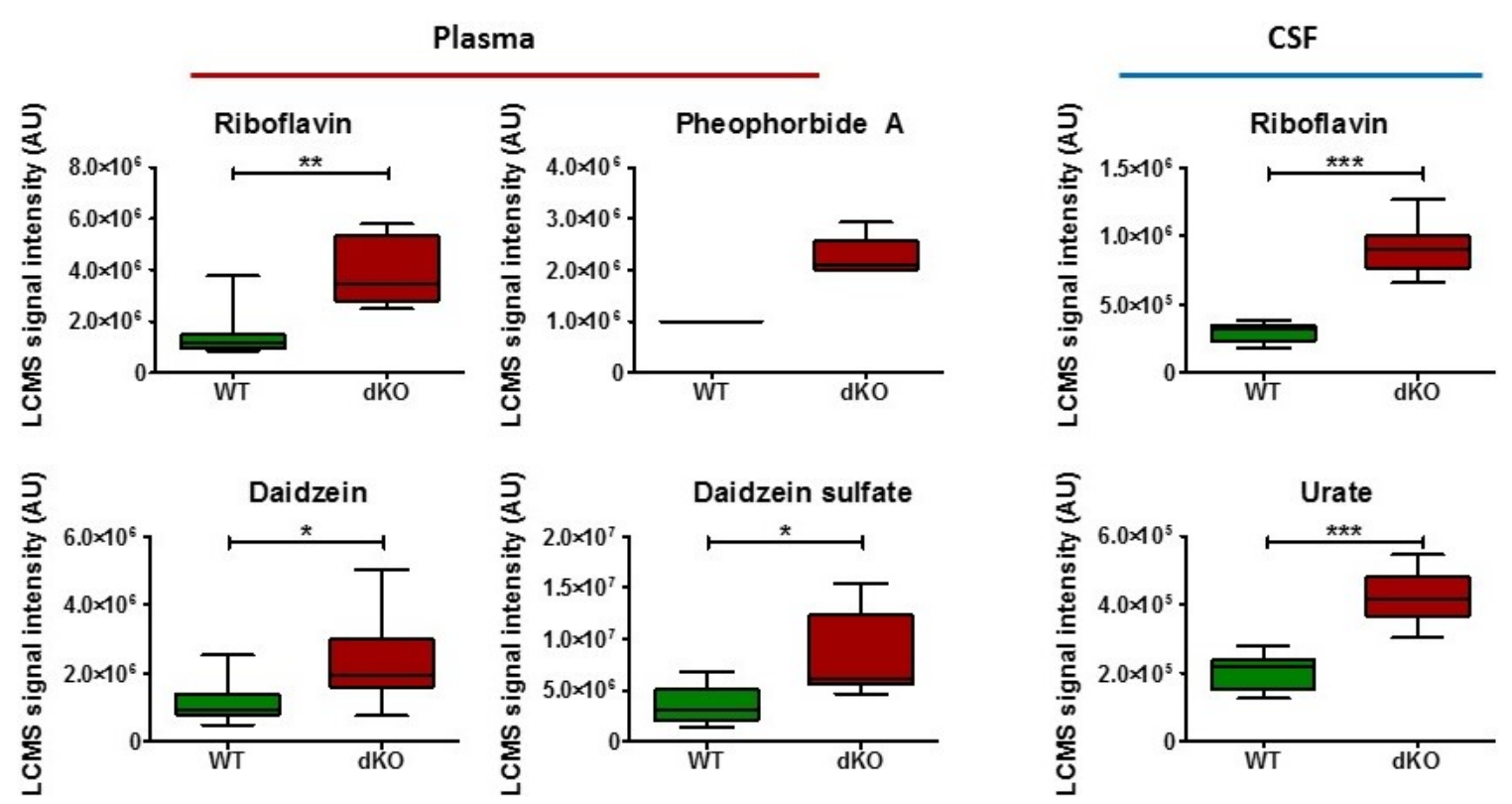

Figure 2-11. Known endogenous substrates of Bcrp were increased in abundance in both plasma and CSF of dKO rats

Note: Raw LCMS median signal intensities are plotted for each genotype $(n=8)$, with significance calculated at $\mathrm{p}<0.05$ by Mann-Whitney test. 
The dietary Bcrp substrate pheophorbide A [92] signal was detected only in the plasma of $\mathrm{dKO}$, but not WT, rats. Another dietary Bcrp substrate the soy isoflavone daidzein (2.05fold) and its metabolite daidzein sulfate (2.41-fold) were also significantly increased in the plasma of $\mathrm{dKO}$ compared to WT rats.

One interesting observation was alteration of few xenobiotics in the $\mathrm{dKO}$ rat plasma (Supplement_Chapter 2_Metabolites Table S5). Among many xenobiotic metabolites ketamine, the anesthetic drug, was found 1.74-fold high in dKO plasma and 1.93-fold high in dKO CSF. Also, a 3.6-fold higher level of 4-hydroxychlorothalonil, a metabolite of pesticide chlorothalonil [219], was found in the plasma of dKO rats compared to WT (Supplement_Chapter 2_Metabolites Table S5).

\section{CSF/plasma intensity ratio identified metabolites with greater CSF} accumulation in $d K O$ rats, compared to WT. Bcrp and Pgp are known to have a significant impact on the brain permeability of their substrates [167] and many Bcrp and Pgp substrates show accumulation in CSF or brain over plasma in the absence or inhibition of these transporters [130, 144, 165]. Therefore, we calculated and compared the CSF/plasma ratio of the raw LC-MS/MS signal intensity of metabolites identified in $\mathrm{WT}$ and $\mathrm{dKO}$ rats. Assuming no significant difference in plasma protein binding between WT and $\mathrm{dKO}$ rats, the above-mentioned ratio of CSF/plasma can be utilized to identify metabolites that have significantly accumulated in the CSF of dKO rats, compared to WT rats. Figure 2-12 shows all metabolites with significantly increased (50 metabolites) (dark red) or decreased (3 metabolites) (dark green) CSF/plasma ratio in dKO vs WT rats. While urate, a known Bcrp substrate, was found to have significantly higher $\mathrm{CSF} /$ plasma ratio (3.23 fold) in dKO compared to WT rats (Figure 2-13), riboflavin, another known substrate of Bcrp, while significantly increased in both plasma and CSF of $\mathrm{dKO}$ rats compared to WT, and had a similar CSF/plasma ratio between WT and $\mathrm{dKO}$ rats (1.11-fold dKO/WT) (Figure 2-14). Other interesting metabolites with greater accumulation in CSF were catechol sulfate, O-methyl catechol sulfate, methionine sulfoxide, isovalerylcarntine and pyridoxate (Figure 2-13). A list of metabolites with significantly altered $\mathrm{CSF} /$ plasma ratios between WT and dKO rats, along with their biological pathway information are provided in Supplement_Chapter

2_Metabolites_Table S2. $60 \%$ of these metabolites were related to amino acids. Phenol sulfate, a known gut microbiome contributed tyrosine metabolite and isovalerate metabolites, which could be contributed by the gut microbiome, were also altered. 13\% of the metabolites were xenobiotic in nature, which may be either contributed by dietary metabolites, or could also be metabolites of dietary fiber metabolism by gut microbiota. Furthermore, metabolites such as catechol sulfate (pyrocatechol sulfate) and O-methyl catechol sulfate maybe of interest as putative Bcrp substrates with 2.13- and 13-fold higher $\mathrm{CSF} /$ plasma ratio in the $\mathrm{dKO}$ rats, compared to WT, respectively. Pyrocatechol sulfate is a known uremic toxin, supporting a role of Bcrp and Pgp transporters in controlling toxin exposure in the brain. Interestingly, while 11-dehydrocorticosterone was unchanged in the CSF and in the plasma of dKO compared to WT rats, a CSF/plasma ratio of 1.4 indicated an increase in CSF abundance of this steroid metabolite in the $\mathrm{dKO}$ rats. Since glucocorticoids are known Pgp substrates [57], 11-dehydrocorticosterone is likely an endogenous Pgp substrate. 


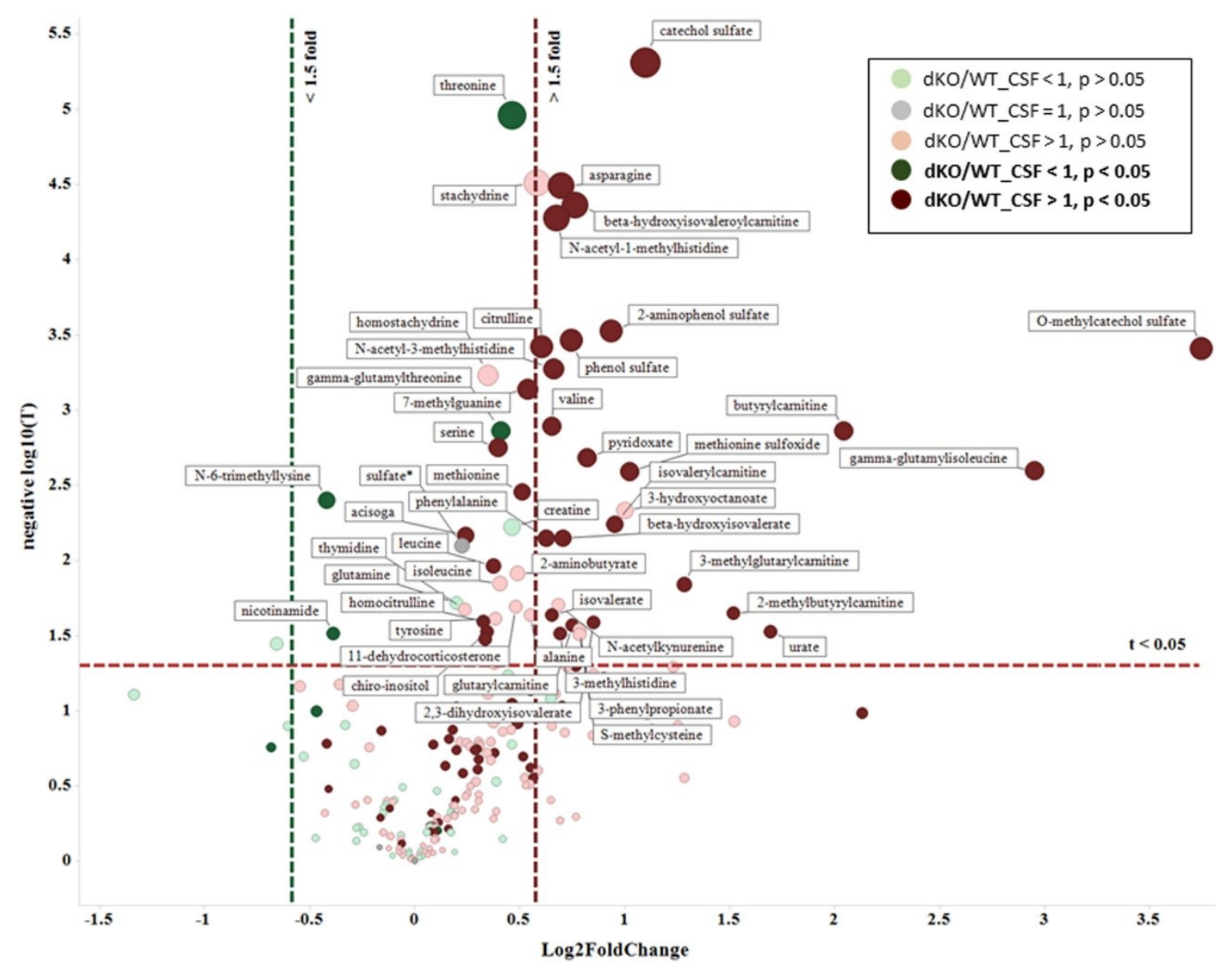

Figure 2-12. Identification of metabolites with significant accumulation in CSF of dKO compared to WT rats: Volcano plot

Notes: Fold change is calculated by dividing CSF/plasma ratio of metabolite raw signal intensity in dKO with that of WT rats. Log2 (Fold change) is plotted on X-axis and log 10 ( $\mathrm{t}$-value of $\mathrm{CSF} /$ plasma $\mathrm{dKO}$ vs $\mathrm{CSF} /$ plasma WT metabolite ratio) is plotted on Yaxis. Metabolites that are significantly higher $(\mathrm{p}<0.05)$ in CSF of dKO over WT rats are identified by dark red marker and metabolites that are significantly decreased $(p<0.05)$ in CSF of dKO over WT rats are identified with dark green marker. Metabolites in dark red, with $>1.5$-fold (red vertical line) significant increase in $\mathrm{CSF} /$ plasma ratio in $\mathrm{dKO}$ compared to WT signifies metabolites with significant accumulation in dKO CSF. 

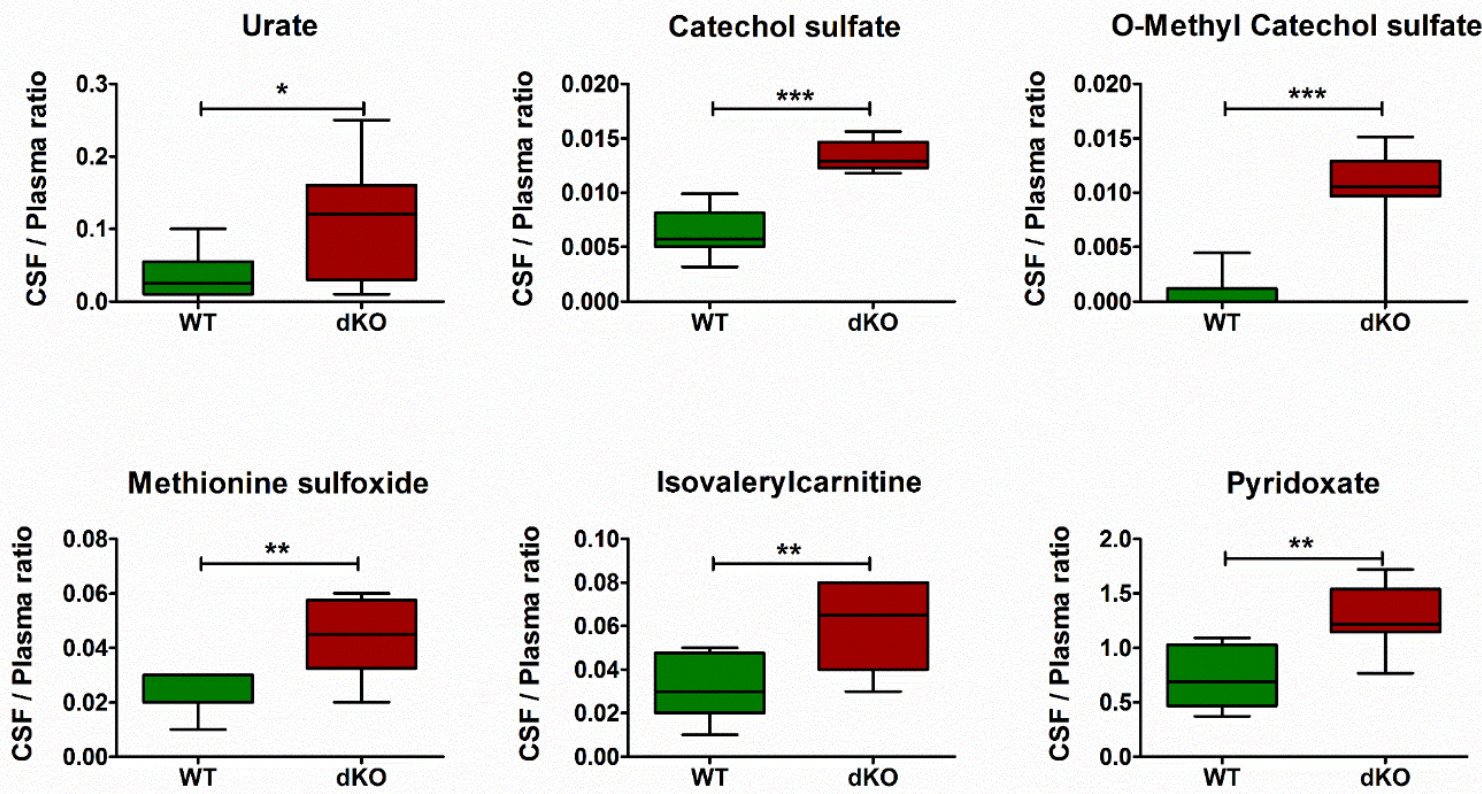

Figure 2-13. Identification of metabolites with significant accumulation in CSF of dKO compared to WT rats

Notes: Box plots of metabolites with significantly higher CSF/plasma ratio in the dKO rats, similar to urate, is plotted as median value \pm range and significance is calculated by comparing the mean CSF/plasma of dKO with WT by two sample t-test assuming unequal variance. 


\section{Riboflavin}
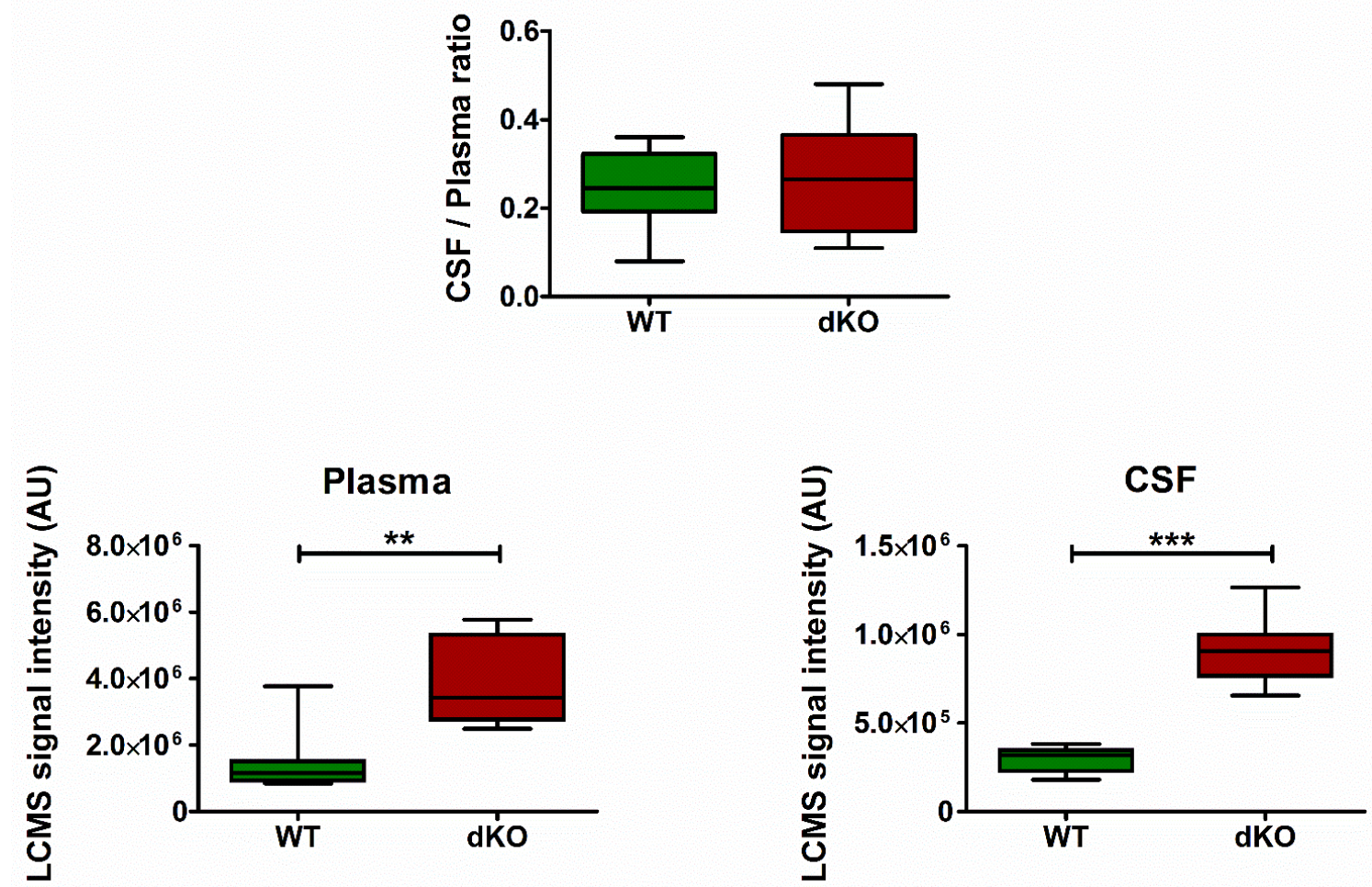

Figure 2-14. Unlike urate riboflavin did not accumulate in CSF

Notes: Box plot of median \pm range of riboflavin intensity in plasma and CSF of $\mathrm{dKO}$ and WT are significantly different, but median \pm range $\mathrm{CSF} /$ plasma ratio is not different between $\mathrm{WT}$ and $\mathrm{dKO}$ rats 
4. Metabolites related to specific metabolic pathways were altered in the CSF and plasma of dKO rats, compared to WT. Apart from significant changes in many metabolites, the following three significantly altered metabolic pathways were of particular interest:

(a) dKO rats show a significantly altered plasma lipid metabolome: Significant alteration in fatty acid metabolites was observed particularly in the plasma metabolome of dKO rats, compared to WT (Table 2-1). While Bcrp or Pgp substrates are expected to increase in abundance in dKO rat plasma, we observed a significant decrease in medium and long chain fatty acids, unsaturated fatty acids, dicarboxylate, and monohydroxy fatty acids, amide and amino fatty acids in the plasma of $\mathrm{dKO}$ rats, indicating a significant alteration in the fatty acid metabolism pathway.

Unlike the fatty acid metabolites, there was a significant increase in the lysolipid metabolites in the plasma (Table 2-2). Similarly, few fatty acid metabolites were also found to increase in abundance in the dKO rat CSF, e.g. butyrylcarnitine showed a $>3.5$ fold increase in CSF abundance in the dKO rats (Table 2-3). The significant increase in few fatty acid metabolites in CSF could be related to Bcrp and/or Pgp mediated transport, as well as possible compensatory transporter expression or metabolic pathway changes.

(b) dKO rats show significant exposure of microbiome, xenobiotic and sulfated metabolites in CSF and plasma: Absence of the Bcrp and Pgp efflux transporters in the intestinal epithelial cells is expected to increase absorption of dietary metabolites as well as gut microbiota metabolites. The metabolomic analysis clearly showed significant alteration of gut microbiota metabolites in both CSF and plasma (Table 2-4). Of particular interest, gut microbiota metabolites of the amino acid tyrosine, p-cresol sulfate and phenol sulfate decreased 0.45 -fold and increases 1.93 -fold, respectively, in the CSF of dKO rats. In addition, sulfated metabolites, many of which are contributed by the gut microbiota, were also altered (Table 2-5 and Table 2-6). Among these metabolites, pcresol sulfate, equol sulfate (daidzein metabolite) and daidzein sulfate are known Bcrp substrates $[170,220]$. Due to high affinity of Bcrp for sulfated metabolites, these metabolites may be of particular interest as putative Bcrp substrates.

(c) Altered purine and pyrimidine biosynthesis pathway metabolites: The purine catabolites xanthosine, urate, allantoin, and allantoic acid, as well as, the pyrimidine degradation products uracil, pseudouridine, and $\mathrm{N}$-acetyl-beta-alanine accumulated in the CSF and plasma of dKO rats (Supplement_Chapter 2_Metabolites_Table S5 and S6). The pyrimidine metabolites orotate and orotidine were also higher in dKO CSF and to less of an extent in plasma compared to WT rats (Supplement_Chapter 2_Metabolites

_Table S5 and S6). Bcrp transporter shows affinity towards purine analogues [221] and thus the alteration in purine pathway metabolites could be the direct result of a lack of Bcrp function. Accumulation of the pyrimidine analogues in the CSF could also indicate these being putative Bcrp and/or Pgp substrates. 
Table 2-1. Decrease in fatty acids in dKO rat plasma

\begin{tabular}{|c|c|c|c|}
\hline Sub Pathway & Biochemical Name & $\begin{array}{c}\text { Fold } \\
\text { Change }\end{array}$ & $\begin{array}{c}\text { p- } \\
\text { Value }\end{array}$ \\
\hline Medium Chain Fatty Acid & heptanoate $(7: 0)$ & 0.79 & 0.08 \\
\hline Medium Chain Fatty Acid & 10 -undecenoate $(11: \ln 1)$ & 0.44 & 0.05 \\
\hline Medium Chain Fatty Acid & 5-dodecenoate $(12: \ln 7)$ & 0.56 & 0.04 \\
\hline Long Chain Fatty Acid & myristoleate $(14: 1 \mathrm{n} 5)$ & 0.64 & 0.06 \\
\hline Long Chain Fatty Acid & eicosenoate $(20: \ln 9$ or 11$)$ & 0.52 & 0.01 \\
\hline Long Chain Fatty Acid & erucate $(22: \ln 9)$ & 0.61 & 0.00 \\
\hline Polyunsaturated Fatty Acid (n3 and n6) & docosahexaenoate (DHA; 22:6n3) & 0.59 & 0.01 \\
\hline Polyunsaturated Fatty Acid (n3 and n6) & docosatrienoate $(22: 3 \mathrm{n} 3)$ & 0.64 & 0.07 \\
\hline Polyunsaturated Fatty Acid (n3 and n6) & arachidonate $(20: 4 n 6)$ & 0.75 & 0.07 \\
\hline Polyunsaturated Fatty Acid (n3 and n6) & adrenate $(22: 4 \mathrm{n} 6)$ & 0.52 & 0.01 \\
\hline Polyunsaturated Fatty Acid (n3 and n6) & $\begin{array}{l}\text { docosapentaenoate (n6 DPA; } \\
22: 5 \mathrm{n} 6 \text { ) }\end{array}$ & 0.42 & 0.00 \\
\hline Polyunsaturated Fatty Acid (n3 and n6) & mead acid $(20: 3 n 9)$ & 0.51 & 0.02 \\
\hline Fatty Acid, Branched & isocaproate & 0.45 & 0.07 \\
\hline Fatty Acid, Branched & 15-methylpalmitate & 0.76 & 0.06 \\
\hline Fatty Acid, Dicarboxylate & azelate (nonanedioate) & 0.76 & 0.08 \\
\hline Fatty Acid, Dicarboxylate & tetradecanedioate & 0.6 & 0.07 \\
\hline Fatty Acid, Dicarboxylate & hexadecanedioate & 0.6 & 0.02 \\
\hline Fatty Acid, Dicarboxylate & octadecanedioate & 0.66 & 0.01 \\
\hline Fatty Acid, Dicarboxylate & eicosanodioate & 0.6 & 0.01 \\
\hline Fatty Acid, Dicarboxylate & docosadioate & 0.68 & 0.01 \\
\hline Fatty Acid, Amide & stearamide & 0.31 & 0.00 \\
\hline Fatty Acid, Amide & erucamide & 0.25 & 0.01 \\
\hline Fatty Acid, Amino & 2-aminoheptanoate & 0.58 & 0.00 \\
\hline Fatty Acid, Amino & 2-aminooctanoate & 0.65 & 0.01 \\
\hline Fatty Acid Metabolism(Acyl Glycine) & N-palmitoyl glycine & 0.54 & 0.01 \\
\hline Fatty Acid Metabolism(Acyl Carnitine) & myristoleoylcarnitine* & 0.71 & 0.05 \\
\hline Carnitine Metabolism & carnitine & 1.14 & 0.03 \\
\hline Fatty Acid, Monohydroxy & 2-hydroxyoctanoate & 0.39 & 0.00 \\
\hline Fatty Acid, Monohydroxy & 2-hydroxydecanoate & 0.45 & 0.00 \\
\hline Fatty Acid, Monohydroxy & 5-hydroxyhexanoate & 0.67 & 0.02 \\
\hline Fatty Acid, Monohydroxy & 16-hydroxypalmitate & 0.7 & 0.02 \\
\hline Fatty Acid, Monohydroxy & 13-HODE + 9-HODE & 0.69 & 0.09 \\
\hline Fatty Acid, Dihydroxy & 12,13-DiHOME & 0.65 & 0.09 \\
\hline Endocannabinoid & oleic ethanolamide & 0.85 & 0.07 \\
\hline Inositol Metabolism & pinitol & 1.67 & 0.01 \\
\hline Phospholipid Metabolism & glycerophosphorylcholine (GPC) & 1.61 & 0.01 \\
\hline Phospholipid Metabolism & glycerophosphoethanolamine & 2.00 & 0.09 \\
\hline Monoacylglycerol & 1-oleoylglycerol (1-monoolein) & 0.52 & 0.04 \\
\hline Sphingolipid Metabolism & palmitoyl sphingomyelin & 1.49 & 0.00 \\
\hline Sterol & 7-Hoca & 0.57 & 0.00 \\
\hline Sterol & campesterol & 1.38 & 0.00 \\
\hline Steroid & corticosterone & 0.64 & 0.00 \\
\hline Steroid & 11-dehydrocorticosterone & 0.8 & 0.07 \\
\hline Primary Bile Acid Metabolism & chenodeoxycholate & 0.51 & 0.10 \\
\hline Primary Bile Acid Metabolism & glycochenodeoxycholate & 0.11 & 0.09 \\
\hline Secondary Bile Acid Metabolism & deoxycholate & 0.55 & 0.06 \\
\hline Secondary Bile Acid Metabolism & glycodeoxycholate & 0.1 & 0.03 \\
\hline Secondary Bile Acid Metabolism & taurodeoxycholate & 0.57 & 0.06 \\
\hline Secondary Bile Acid Metabolism & tauroursodeoxycholate & 0.58 & 0.08 \\
\hline Secondary Bile Acid Metabolism & taurocholenate sulfate & 1.99 & 0.01 \\
\hline
\end{tabular}




\section{Table 2-1. Continued}

Note: Raw signal intensity of the metabolites was used to compute the fold change $(\mathrm{dKO} /$ WT) and p-value was calculated using Welch's two sample t-test to identify metabolites as significant $(\mathrm{p}<0.05)$ (in dark green $\&$ red) or trending significant $(0.1>\mathrm{p}>0.05)$ (light green \& red) metabolites. 
Table 2-2. Increase in lysolipids in dKO rat plasma

\begin{tabular}{llcc}
\hline Sub Pathway & Biochemical Name & Fold Change & p-Value \\
\hline Lysolipid & 1-stearoylglycerophosphocholine (18:0) & $\mathbf{0 . 7 7}$ & 0.03 \\
Lysolipid & 2-stearoylglycerophosphocholine & $\mathbf{0 . 6 3}$ & 0.06 \\
Lysolipid & 1-linoleoylglycerophosphocholine (18:2n6) & $\mathbf{1 . 0 9}$ & 0.00 \\
Lysolipid & 1-arachidonoylglycerophosphocholine (20:4n6) & $\mathbf{0 . 9 1}$ & 0.08 \\
Lysolipid & 1-oleoylglycerophosphoethanolamine & $\mathbf{1 . 3 9}$ & 0.00 \\
Lysolipid & 1-linoleoylglycerophosphoethanolamine & $\mathbf{1 . 3 7}$ & 0.01 \\
Lysolipid & 1-palmitoylglycerophosphoinositol & $\mathbf{2 . 0 9}$ & 0.01 \\
Lysolipid & 1-linoleoylglycerophosphoinositol & $\mathbf{2 . 1 7}$ & 0.01 \\
Lysolipid & oleoyl-linoleoyl-glycerophosphoinositol (1) & $\mathbf{1 . 4 3}$ & 0.00 \\
Lysolipid & palmitoyl-linoleoyl-glycerophosphocholine (1) & $\mathbf{1 . 3 4}$ & 0.00 \\
Lysolipid & palmitoyl-linoleoyl-glycerophosphocholine (2) & $\mathbf{1 . 3 3}$ & 0.00 \\
Lysolipid & palmitoyl-linoleoyl-glycerophosphoinositol (1) & $\mathbf{1 . 7}$ & 0.00 \\
Lysolipid & palmitoyl-oleoyl-glycerophosphocholine (1) & $\mathbf{1 . 2 4}$ & 0.01 \\
Lysolipid & stearoyl-arachidonoyl-glycerophosphocholine (2) & $\mathbf{0 . 6 8}$ & 0.00 \\
Lysolipid & oleoyl-linoleoyl-glycerophosphocholine (1) & $\mathbf{1 . 1 8}$ & 0.01 \\
Lysolipid & oleoyl-linoleoyl-glycerophosphocholine (2) & $\mathbf{1 . 3 9}$ & 0.04 \\
Lysolipid & stearoyl-arachidonoyl-glycerophosphoethanolamine (1) & $\mathbf{1 . 6 8}$ & 0.00 \\
Lysolipid & stearoyl-linoleoyl-glycerophosphoethanolamine (1) & $\mathbf{2 . 1 8}$ & 0.00 \\
Lysolipid & 2-behenoylglycerophosphocholine & $\mathbf{0 . 2 4}$ & 0.02 \\
Lysolipid & stearoyl-arachidonoyl-glycerophosphoinositol (2) & $\mathbf{0 . 8 7}$ & 0.01 \\
\hline
\end{tabular}

Note: Fold change (dKO/ WT) was computed from raw signal intensity of metabolites and Welch's two sample t-test was used to identify metabolites as significant $(\mathrm{p}<0.05)$ (in dark green $\&$ red) or trending significant $(0.1>\mathrm{p}>0.05)$ (light green $\&$ red). 
Table 2-3. Increase in fatty acids in dKO rat CSF

\begin{tabular}{|c|c|c|c|c|c|}
\hline \multirow[b]{2}{*}{ Sub Pathway } & \multirow[b]{2}{*}{ Biochemical Name } & \multicolumn{2}{|c|}{$\begin{array}{c}\text { Fold Change } \\
\text { (dKO/WT) }\end{array}$} & \multicolumn{2}{|c|}{ p-Value } \\
\hline & & $\begin{array}{c}\text { All } \\
\text { Sample }\end{array}$ & $\begin{array}{l}\text { No Cont. } \\
\text { Sample }\end{array}$ & $\begin{array}{c}\text { All } \\
\text { Sample }\end{array}$ & $\begin{array}{l}\text { No Cont. } \\
\text { Sample }\end{array}$ \\
\hline $\begin{array}{l}\text { Fatty Acid, } \\
\text { Dicarboxylate }\end{array}$ & $\begin{array}{l}\text { dimethylmalonic } \\
\text { acid }\end{array}$ & 1.36 & 1.42 & 0.03 & 0.05 \\
\hline Fatty Acid Synthesis & malonylcarnitine & 1.24 & 1.33 & 0.02 & 0.01 \\
\hline Fatty Acid Synthesis & $\begin{array}{l}\text { 2-methylmalonyl } \\
\text { carnitine }\end{array}$ & 1.43 & 1.60 & 0.00 & 0.00 \\
\hline $\begin{array}{l}\text { Fatty Acid } \\
\text { Metabolism (also } \\
\text { BCAA Metabolism) }\end{array}$ & butyrylcarnitine & 3.77 & 3.84 & 0.00 & 0.00 \\
\hline Carnitine Metabolism & deoxycarnitine & 1.42 & 1.41 & 0.00 & 0.00 \\
\hline Carnitine Metabolism & carnitine & 1.18 & 1.14 & 0.09 & 0.18 \\
\hline Ketone Bodies & $\begin{array}{l}\text { 3-hydroxybutyrate } \\
\text { (BHBA) }\end{array}$ & 0.80 & 0.75 & 0.04 & 0.02 \\
\hline Inositol Metabolism & chiro-inositol & 1.51 & 1.70 & 0.02 & 0.01 \\
\hline $\begin{array}{l}\text { Mevalonate } \\
\text { Metabolism }\end{array}$ & $\begin{array}{l}\text { 3-hydroxy-3- } \\
\text { methylglutarate }\end{array}$ & 0.83 & 0.82 & 0.07 & 0.04 \\
\hline Sterol & $\begin{array}{l}\text { 7-alpha-hydroxy-3- } \\
\text { oxo-4-cholestenoate } \\
\text { (7-Hoca) }\end{array}$ & 0.56 & 0.69 & 0.05 & 0.18 \\
\hline
\end{tabular}

Notes: Fold change (dKO/ WT) was computed from raw signal intensity of metabolites and Welch's two sample t-test was used to identify metabolites as significant $(\mathrm{p}<0.05)$ (in dark green $\&$ red) or trending significant $(0.1>p>0.05)$ (light green \& red). CSF samples were analyzed without contamination compensation or with contamination compensation (No Cont. Sample). 
Table 2-4. Gut microbiota metabolites identified altered in the plasma or CSF of dKO rats

\begin{tabular}{|c|c|c|c|c|c|c|}
\hline \multirow[b]{2}{*}{ Metabolites } & \multicolumn{2}{|c|}{ Plasma } & \multicolumn{2}{|c|}{ CSF } & \multirow[b]{2}{*}{$\begin{array}{c}\text { Reference } \\
\text { (PMID) }\end{array}$} & \multirow[b]{2}{*}{$\begin{array}{c}\text { Exclusive } \\
\text { Biome? }\end{array}$} \\
\hline & $\begin{array}{c}\text { Fold } \\
\text { Change }\end{array}$ & $\begin{array}{c}\text { p- } \\
\text { Value }\end{array}$ & $\begin{array}{c}\text { Fold } \\
\text { Change }\end{array}$ & $\begin{array}{c}\text { p- } \\
\text { Value }\end{array}$ & & \\
\hline riboflavin (Vitamin B2) & 2.64 & 0.0002 & 3.09 & $2.00 \mathrm{E}-$ & 26283345 & \\
\hline creatine & 0.7 & 0.0016 & 0.95 & 0.35 & 26283345 & \\
\hline 4-hydroxycinnamate & 0.53 & 0.0023 & & & 26861391 & \\
\hline taurocholenate sulfate & 1.99 & 0.009 & & & 26283345 & $\mathrm{Y}$ \\
\hline p-cresol sulfate & 0.45 & 0.0091 & & & 26283345 & $\mathrm{Y}$ \\
\hline phenyllactate (PLA) & 1.72 & 0.0117 & 1.56 & 0.0004 & 27505423 & \\
\hline daidzein & 2.05 & 0.0181 & & & & \\
\hline indolelactate & 1.45 & 0.0201 & & & 26283345 & $\mathrm{Y}$ \\
\hline phenylacetylglutamine & 0.46 & 0.0215 & & & 26283345 & $\mathrm{Y}$ \\
\hline glycodeoxycholate & 0.1 & 0.0309 & & & 26283345 & $\mathrm{Y}$ \\
\hline $\begin{array}{l}3 \text {-(2-hydroxyphenyl) } \\
\text { propionate }\end{array}$ & 0.62 & 0.0314 & & & 27505423 & \\
\hline phenylacetate & 0.44 & 0.0338 & & & & \\
\hline indoleacetate & 0.75 & 0.0539 & 1.53 & 0.14 & 26283345 & Y \\
\hline deoxycholate & 0.55 & 0.0607 & & & & \\
\hline indole-3-carboxylic & 0.64 & 0.0742 & & & & \\
\hline tauroursodeoxycholate & 0.58 & 0.0769 & & & 26283345 & Y \\
\hline phenol sulfate & 1.27 & 0.1189 & 1.93 & 0.0078 & 26283345 & $\mathrm{Y}$ \\
\hline Isovalerate & 0.86 & 0.3158 & 1.25 & 0.0084 & 26283345 & $\mathrm{~N}$ \\
\hline creatinine & 1.07 & 0.4987 & 1.21 & 0.0005 & & \\
\hline
\end{tabular}

Notes: Metabolites of gut microbiota origin were identified based on previously published papers as well as microbiome metabolite list maintained at Metabolon ${ }^{\circledR}$. Only significant $(\mathrm{p}<0.05)$ and trending significant $(0.1>\mathrm{p}>0.05)$ metabolites are listed from both plasma and CSF. Fold change is the ratio of $\mathrm{dKO} / \mathrm{WT}$ metabolite signal intensity and Welch's two sample t-test is used to compare WT vs dKO and calculate significance. 
Table 2-5. Sulfated metabolites significantly altered in the plasma of dKO rats

\begin{tabular}{llcc}
\hline Sub Pathway & Biochemical Name & $\begin{array}{c}\text { Fold } \\
\text { Change }\end{array}$ & p-Value \\
\hline Phenylalanine and Tyrosine Metabolism & p-cresol sulfate & $\mathbf{0 . 4 5}$ & 0.01 \\
Secondary Bile Acid Metabolism & taurocholenate sulfate & $\mathbf{1 . 9 9}$ & 0.01 \\
Benzoate Metabolism & catechol sulfate & $\mathbf{1 . 4}$ & 0.08 \\
Food Component/Plant & equol sulfate & $\mathbf{1 . 5 7}$ & 0.02 \\
Food Component/Plant & 4-vinylguaiacol sulfate & $\mathbf{0 . 4 1}$ & 0.04 \\
Food Component/Plant & daidzein sulfate (2) & $\mathbf{2 . 4 1}$ & 0.00 \\
Drug & 4-acetylphenol sulfate & $\mathbf{2 . 2 9}$ & 0.00 \\
Chemical & sulfate* & $\mathbf{0 . 8 7}$ & 0.07 \\
Chemical & 2-aminophenol sulfate & $\mathbf{1 . 4 4}$ & 0.05 \\
Chemical & 3-hydroxypyridine sulfate & $\mathbf{2 . 5 3}$ & 0.00 \\
\hline
\end{tabular}

Notes: Sulfated metabolites of different pathway origin that were significantly increased or decreased in dKO rat plasma are listed. Welch's two sample t-test was used to identify metabolites as significant $(\mathrm{p}<0.05)$ (in dark green $\&$ red) or trending significant $(0.1>\mathrm{p}>0.05)$ (light green $\&$ red) metabolites.

Table 2-6. Sulfated metabolites significantly altered in the CSF of dKO rats

\begin{tabular}{|c|c|c|c|c|c|}
\hline \multirow[b]{2}{*}{ Sub Pathway } & \multirow[b]{2}{*}{$\begin{array}{l}\text { Biochemical } \\
\text { Name }\end{array}$} & \multicolumn{2}{|c|}{ Fold Change (dKO/WT) } & \multicolumn{2}{|c|}{ p-Value } \\
\hline & & All Sample & $\begin{array}{l}\text { No Cont. } \\
\text { Sample }\end{array}$ & $\begin{array}{c}\text { All } \\
\text { Sample }\end{array}$ & $\begin{array}{l}\text { No Cont } \\
\text { Sample }\end{array}$ \\
\hline $\begin{array}{l}\text { Phenylalanine and } \\
\text { Tyrosine Metabolism }\end{array}$ & phenol sulfate & 1.93 & 1.84 & 0.01 & 0.04 \\
\hline Dipeptide & prolylglycine & 1.17 & 1.15 & 0.02 & 0.06 \\
\hline Benzoate Metabolism & catechol sulfate & 3.05 & 3.31 & 0.00 & 0.00 \\
\hline Benzoate Metabolism & $\begin{array}{l}\text { O-methylcatechol } \\
\text { sulfate }\end{array}$ & 4.04 & 5.52 & 0.00 & 0.00 \\
\hline Chemical & $\begin{array}{l}\text { 2-aminophenol } \\
\text { sulfate }\end{array}$ & 2.71 & 2.93 & 0.00 & 0.00 \\
\hline
\end{tabular}

Notes: Sulfated metabolites of different pathway origin that were significantly increased or decreased in $\mathrm{dKO}$ rat CSF are listed. Welch's two sample t-test was used to identify metabolites as significant $(\mathrm{p}<0.05)$ (in dark green $\&$ red) or trending significant $(0.1>p>0.05)$ (light green $\&$ red) . 


\section{Bcrp and Pgp gene deletion in rats results in altered transcriptome of brain, kidney and liver}

To understand the effect of compensatory pathways on metabolomic signatures, we performed a microarray analysis comparing gene expression in the brain (frontal cortex), kidney and liver of WT and dKO rats. A total of 435 genes in kidney, 398 genes in liver and 240 genes in brain frontal cortex was found to be significantly different (Figure 2-15A). 75 genes were common between kidney and liver, 45 genes between kidney and brain and 40 genes between brain and liver. Hierarchical clustering analysis revealed striking differences in gene expression in all tissues between the WT and $\mathrm{dKO}$ rats (Figure 2-15B). A list of significantly altered genes in all tissues, along with their associated $\mathrm{p}$-values, identified by both Nexus expression analysis and GSEA analysis method are provided in the supplement (Supplement_Chapter 2_Genes. S7 and S8) Gene expression analysis exhibited significant changes in gene expression of few Cytochrome P450 enzymes (Table 2-7), few ABC efflux transporters, mainly the Abcg group of transporters (Table 2-8) and few solute carrier (SLC) uptake transporters (Table 2-9), but none of the major renal drug efflux or uptake transporters. A significant increase (2.93-fold) in Cyp2b1 gene expression was observed in the liver of dKO rats, compared to WT rats. Many SLC uptake transporters in kidney and liver were also found to have altered expression (Table 2-9), e.g. Slc22a5 or OCTN2 is a carnitine transporter, significantly decreased in the kidney of dKO rats. Similarly, an alteration in the Abcg group of transporters in kidney (Abcg1, 1.5-fold) and liver (Abcg3, 0.5-fold and Abcg1, 1.5 -fold) indicates compensatory alteration of these transporters due to absence of Abcg2 or Bcrp. Upregulation of Abcg1, sterol and choline phospholipid transporter [14], might have an impact on steroid and sterol metabolism thus impacting the lipid metabolome. Cholesterol and steroid metabolic pathway was also found to be altered in the kidney and liver of dKO rats, respectively (Table 2-10). Table 2-10 lists the significantly altered top 20 common biological pathways between brain, liver and kidney of, dKO rats, compared to WT rats. Of particular interest among altered pathways were urate biosynthetic process and response to lipopolysaccharide in brain, oxidation-reduction process and response to oxidative stress in kidney and circadian rhythm in liver, a majority of which were found to be altered in all three tissues (Table 2-11). These altered pathway information, in conjunction with the metabolomic signatures, indicated a possible impact of Bcrp and Pgp transporters at the physiological level.

\section{Use of gene expression and known substrate knowledge for understanding metabolomic features}

Significantly altered biological pathways and the associated gene expression changes indicated that a part of the observed metabolomic changes was due to either direct effect of compensatory pathways or indirect effect of many metabolites. Figure 2-16, Figure 2-17 and Figure 2-18 show few proposed models of possible genemetabolite interactions and impact of Bcrp and/or Pgp on biological pathway, respectively, derived from a knowledge-based integration of metabolite-gene expressionbiological pathway alterations. 
$\mathbf{A}$

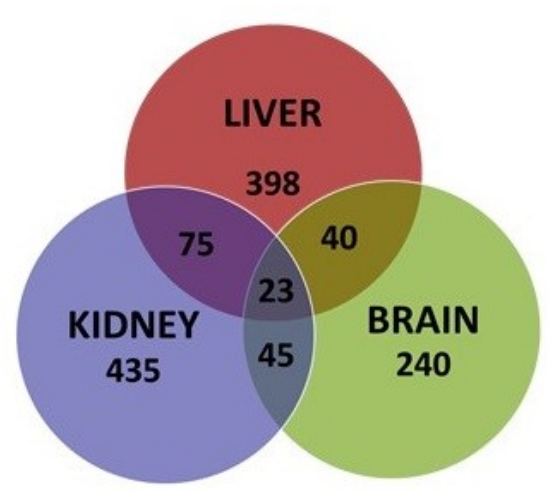

$\mathbf{B}$

LIVER

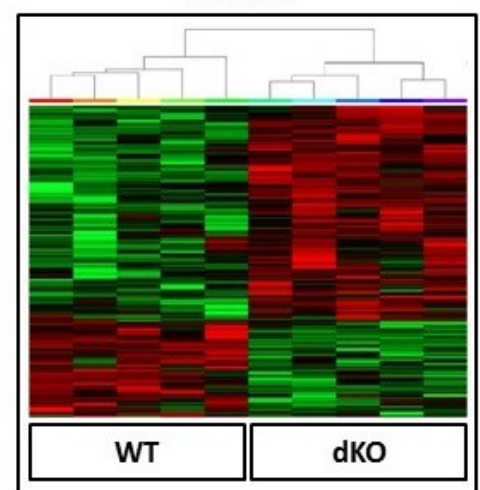

KIDNEY

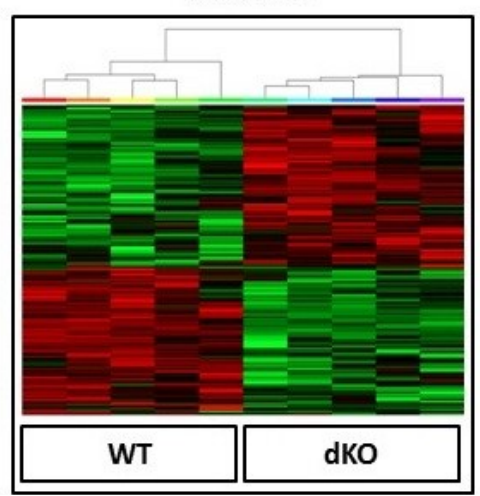

BRAIN

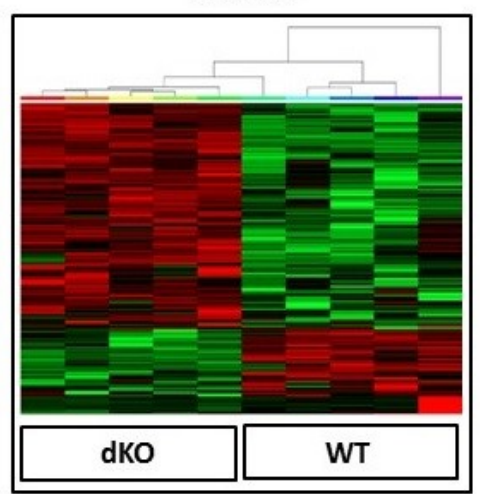

Figure 2-15. Gene expression in brain, kidney and liver of the dKO is significantly different from the WT rats

Notes: Venn diagram showing the number of significant $(\mathrm{p}<0.05)$ genes in kidney (blue), liver (red) and brain frontal cortex (green) of the dKO compared to the WT rats (A). Hierarchical clustering heatmap shows significant expression in gene expression between WT and $\mathrm{dKO}$ rat kidney, liver and brain frontal cortex tissues (B) 
Table 2-7. Significantly changed Cytochrome P450 (CYP) enzymes in dKO rats

\begin{tabular}{|c|c|c|c|c|c|c|c|}
\hline \multirow[b]{2}{*}{$\begin{array}{l}\text { Gene } \\
\text { Symbol }\end{array}$} & \multirow[b]{2}{*}{ Regulation } & \multicolumn{2}{|c|}{ Liver } & \multicolumn{2}{|c|}{ Brain } & \multicolumn{2}{|c|}{ Kidney } \\
\hline & & $\begin{array}{c}\text { Fold } \\
\text { Change }\end{array}$ & p-Value & $\begin{array}{c}\text { Fold } \\
\text { Change }\end{array}$ & p-Value & $\begin{array}{c}\text { Fold } \\
\text { Change }\end{array}$ & p-Value \\
\hline Cyp4v3 & UP & & & 1.33 & 0.02 & 1.24 & 0.01 \\
\hline Сур4а8 & DOWN & 0.71 & 0.00 & & & & \\
\hline Сур4а3 & UP & & & & & 1.50 & 0.00 \\
\hline Сур3а85-ps & DOWN & 0.58 & 0.00 & & & & \\
\hline Сур3а73 & DOWN & 0.67 & 0.00 & & & & \\
\hline Сур2j4 & UP & 1.34 & 0.01 & & & 1.61 & 0.00 \\
\hline Cyp2g1 & DOWN & & & 0.72 & 0.04 & & \\
\hline Cyp2d5 & DOWN & 0.54 & 0.00 & & & 0.33 & 0.00 \\
\hline Сур2d2 & DOWN & & & & & 0.75 & 0.00 \\
\hline Сур2d1 & DOWN & & & & & 0.71 & 0.00 \\
\hline Сур2с12 & DOWN & 0.56 & 0.00 & & & & \\
\hline Сур2c11 & UP & & & & & 1.77 & 0.00 \\
\hline Сур2b2 & UP & 1.24 & 0.04 & & & & \\
\hline Сур2b1 & UP & 2.66 & 0.00 & & & & \\
\hline Сур26а1 & UP & 1.39 & 0.01 & & & & \\
\hline Сур24a1 & UP & & & & & 1.79 & 0.00 \\
\hline Cyp1a1 & UP & & & & & 1.76 & 0.00 \\
\hline
\end{tabular}

Note: List of significantly $(\mathrm{p}<0.05)$ increased (red) or decreased (green) CYP enzymes' gene expression in the $\mathrm{dKO}$ rats.

Table 2-8. Significantly changed ATP-binding Cassette (ABC) transporters in dKO rats

\begin{tabular}{|c|c|c|c|c|c|c|c|}
\hline \multirow[b]{2}{*}{$\begin{array}{l}\text { Gene } \\
\text { Symbol }\end{array}$} & \multirow[b]{2}{*}{ Regulation } & \multicolumn{2}{|c|}{ Liver } & \multicolumn{2}{|c|}{ Brain } & \multicolumn{2}{|c|}{ Kidney } \\
\hline & & $\begin{array}{c}\text { Fold } \\
\text { Change }\end{array}$ & p-Value & $\begin{array}{c}\text { Fold } \\
\text { Change }\end{array}$ & p-Value & $\begin{array}{c}\text { Fold } \\
\text { Change }\end{array}$ & p-Value \\
\hline Abca16 & DOWN & 0.65 & 0.00 & & & & \\
\hline Abca9 & UP & 1.35 & 0.00 & & & & \\
\hline Abcc10 & DOWN & 0.73 & 0.01 & & & & \\
\hline Abcg1 & UP & & & & & 1.48 & 0.00 \\
\hline Abcg3 & DOWN & 0.47 & 0.00 & & & & \\
\hline Abcg313 & DOWN & & & & & 0.76 & 0.02 \\
\hline Abcg5 & UP & 1.50 & 0.00 & & & & \\
\hline
\end{tabular}

Note: List of significantly $(\mathrm{p}<0.05)$ increased (red) or decreased (green) ABC transporters' gene expression in the $\mathrm{dKO}$ rats. 
Table 2-9. Significantly changed SLC transporters in dKO rats

\begin{tabular}{|c|c|c|c|c|c|c|c|c|c|}
\hline \multirow[b]{2}{*}{$\begin{array}{l}\text { Gene } \\
\text { Symbol }\end{array}$} & \multirow[b]{2}{*}{$\begin{array}{l}\text { Common } \\
\text { Name }\end{array}$} & \multirow[b]{2}{*}{ Regulation } & \multicolumn{2}{|c|}{ Liver } & \multicolumn{2}{|c|}{ Brain } & \multicolumn{2}{|c|}{ Kidney } & \multirow[b]{2}{*}{$\begin{array}{l}\text { Possible } \\
\text { Substrates }\end{array}$} \\
\hline & & & $\begin{array}{c}\text { Fold } \\
\text { Change }\end{array}$ & $\begin{array}{c}\text { p- } \\
\text { Value }\end{array}$ & $\begin{array}{c}\text { Fold } \\
\text { Change }\end{array}$ & $\begin{array}{c}\text { p- } \\
\text { Value }\end{array}$ & $\begin{array}{c}\text { Fold } \\
\text { Change }\end{array}$ & $\begin{array}{c}\text { p- } \\
\text { Value }\end{array}$ & \\
\hline Slco1c1 & OATP1C1 & UP & & & 1.22 & 0.02 & & & $\mathrm{~T} 4, \mathrm{~T} 3$ \\
\hline Slc8a1 & & UP & & & & & 1.26 & 0.01 & \\
\hline Slc6a6 & TauT & UP & 1.28 & 0.00 & & & & & Taurine \\
\hline Slc5a8 & SMCT1 & DOWN & & & & & 0.69 & 0.00 & $\begin{array}{l}\text { Short chain } \\
\text { fatty acid }\end{array}$ \\
\hline Slc5a3 & SMIT1 & UP & & & & & 1.28 & 0.02 & Myoinositol \\
\hline Slc5a10 & SGLT5 & DOWN & & & & & 0.80 & 0.04 & $\begin{array}{l}\text { Mannose, } \\
\text { fructose, } \\
\text { glucose }\end{array}$ \\
\hline Slc4a7 & & UP & & & 1.29 & 0.00 & & & \\
\hline Slc4a5 & & UP & & & & & 1.33 & 0.00 & \\
\hline Slc45a3 & & UP & 1.28 & 0.05 & & & & & \\
\hline Slc39a12 & & UP & & & 1.61 & 0.00 & & & \\
\hline Slc38a3 & & UP & & & & & 1.24 & 0.00 & \\
\hline Slc38a2 & & UP & 1.48 & 0.00 & & & & & \\
\hline Slc35f1 & & DOWN & & & 0.83 & 0.04 & 0.65 & 0.00 & \\
\hline Slc34a2 & & UP & 1.39 & 0.00 & & & & & \\
\hline Slc30a2 & & DOWN & & & & & 0.82 & 0.04 & \\
\hline Slc2a12 & Glut12 & DOWN & 0.50 & 0.00 & & & & & Glucose \\
\hline Slc26a11 & & DOWN & & & & & 0.63 & 0.00 & \\
\hline Slc $25 \mathrm{a} 30$ & & DOWN & 0.51 & 0.00 & & & & & \\
\hline Slc $25 \mathrm{a} 28$ & Mitoferrin & DOWN & & & & & 0.82 & 0.05 & $\mathrm{Fe}^{2+}$ \\
\hline $\operatorname{Slc} 25 \mathrm{a} 25$ & & UP & & & & & 1.52 & 0.00 & \\
\hline Slc25a23 & APC2 & DOWN & & & & & 0.82 & 0.00 & $\begin{array}{l}\text { ATP, AMP, } \\
\text { ADP, Pi }\end{array}$ \\
\hline Slc22a5 & OCTN2 & DOWN & 0.78 & 0.01 & & & & & L-carnitine \\
\hline Slc $22 \mathrm{a} 25$ & & DOWN & 0.68 & 0.03 & & & & & \\
\hline Slc16a6 & MCT7 & UP & & & 1.28 & 0.01 & 0.71 & 0.00 & Orphan \\
\hline Slc16a2 & МCT8 & DOWN & & & & & 0.83 & 0.02 & $\mathrm{~T} 2, \mathrm{~T} 3, \mathrm{~T} 4$ \\
\hline Slc15a1 & PEPT1 & DOWN & & & & & 0.59 & 0.00 & $\begin{array}{l}\text { di- and tri- } \\
\text { peptides }\end{array}$ \\
\hline Slc13a5 & $\mathrm{NaC} 2$ & UP & 1.36 & 0.00 & & & & & $\begin{array}{l}\text { Citrate, } \\
\text { succinate, } \\
\text { pyruvate }\end{array}$ \\
\hline
\end{tabular}

Note: List of significantly $(\mathrm{p}<0.05)$ increased (red) or decreased (green) SLC transporters gene expression (Table 2-9) in the dKO rats. 
Table 2-10. Significantly altered top biological pathways based on gene expression changes in dKO compared to WT rats

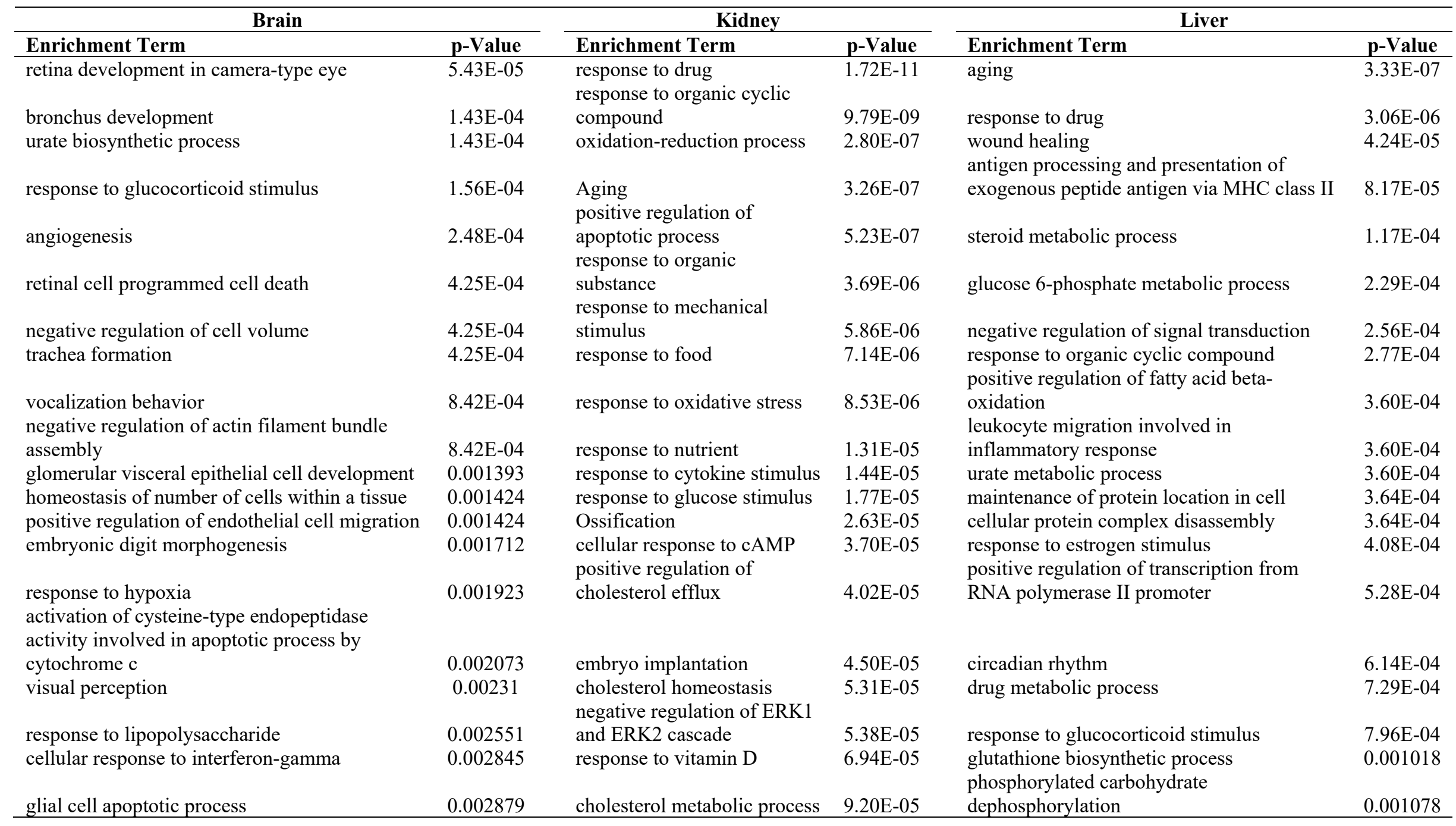

Notes: Top 20 most significant biological pathways were identified by enrichment of significantly altered genes in the dKO rat brain frontal cortex, kidney and liver at $\mathrm{p}<0.05$. 
Table 2-11. Significantly altered common biological pathways between all the tissues

\begin{tabular}{|c|c|c|c|}
\hline \multirow[b]{2}{*}{ Common Biological Pathways } & \multicolumn{3}{|c|}{ p-Value } \\
\hline & Brain & Liver & Kidney \\
\hline urate biosynthetic process & $1.43 \mathrm{E}-04$ & 0.037835 & 0.042625 \\
\hline response to glucocorticoid stimulus & $1.56 \mathrm{E}-04$ & 0.000796 & 0.000459 \\
\hline Angiogenesis & $2.48 \mathrm{E}-04$ & 0.047942 & 0.002913 \\
\hline response to lipopolysaccharide & 0.002551 & 0.013290 & 0.003599 \\
\hline circadian rhythm & 0.004231 & 0.000614 & 0.000171 \\
\hline drug transport & 0.004859 & 0.011985 & 0.015081 \\
\hline antigen processing and presentation & 0.005943 & 0.020920 & 0.028569 \\
\hline drug transmembrane transport & 0.006026 & 0.014794 & 0.018585 \\
\hline antigen processing and presentation of exogenous peptide antigen via MHC class II & 0.010200 & $8.17 \mathrm{E}-05$ & 0.000130 \\
\hline protein localization in mitochondrion & 0.011969 & 0.019100 & 0.021544 \\
\hline cellular response to type I interferon & 0.011969 & 0.019100 & 0.021544 \\
\hline negative regulation of helicase activity & 0.011969 & 0.019100 & 0.021544 \\
\hline positive regulation of angiogenesis & 0.013703 & 0.003538 & 0.001348 \\
\hline male gonad development & 0.020563 & 0.006432 & 0.039839 \\
\hline mitochondrial electron transport; cytochrome $\mathrm{c}$ to oxygen & 0.023795 & 0.037835 & 0.042625 \\
\hline nucleotide-binding oligomerization domain containing 1 signaling pathway & 0.023795 & 0.037835 & 0.042625 \\
\hline hypoxanthine biosynthetic process & 0.023795 & 0.037835 & 0.042625 \\
\hline regulation of mitochondrial fission & 0.023795 & 0.037835 & 0.042625 \\
\hline negative regulation of apoptotic process & 0.039936 & 0.001377 & 0.001713 \\
\hline brown fat cell differentiation & 0.046865 & 0.017487 & 0.000351 \\
\hline response to peptide hormone stimulus & 0.046952 & 0.001474 & 0.000167 \\
\hline intracellular transport of viral proteins in host cell & 0.047027 & 0.002128 & 0.002700 \\
\hline
\end{tabular}

Note: Top 22 common biological pathways that were identified by enrichment of significantly altered genes in the dKO rat brain frontal cortex, kidney and liver at $\mathrm{p}<0.05$. 
A

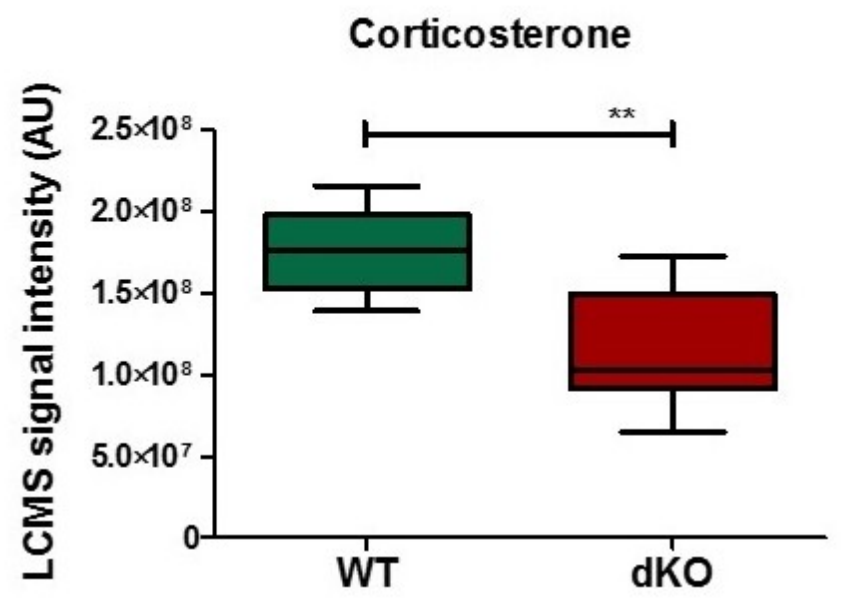

B

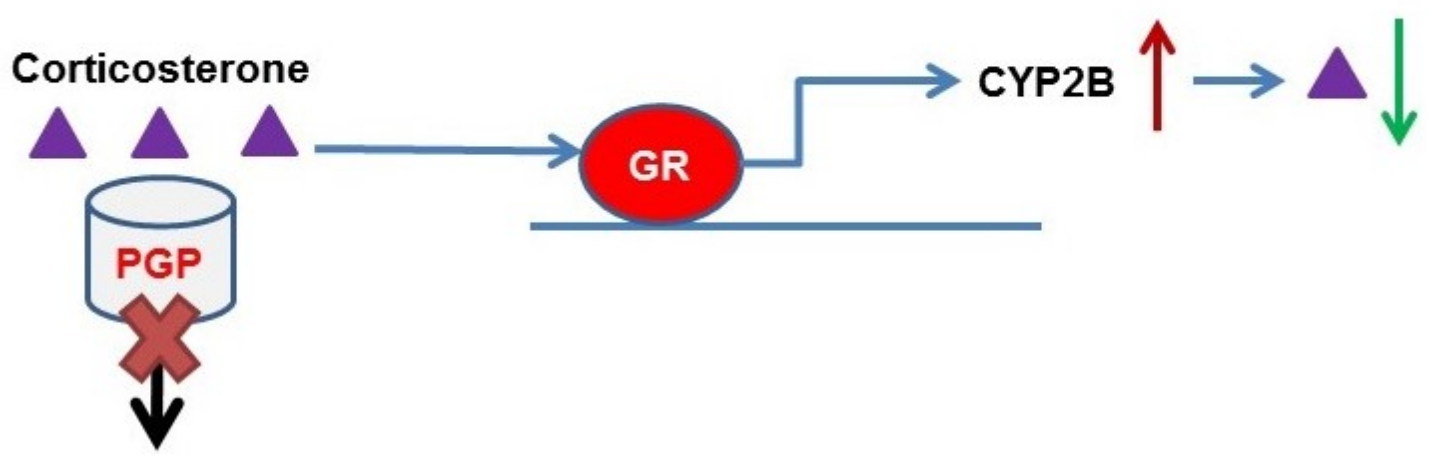

Figure 2-16. Possible explanation of observed decrease in corticosterone, a Pgp substrate, in the $\mathrm{dKO}$ rat plasma

Notes: Metabolomic analysis showed a significant decrease in corticosterone in $\mathrm{dKO}$ rat plasma (A). Transcriptomic analysis showed a significant increase in CYP2B gene expression in the liver of $\mathrm{dKO}$ rats, possibly by glucocorticoid mediated pathway activated by corticosterone. 


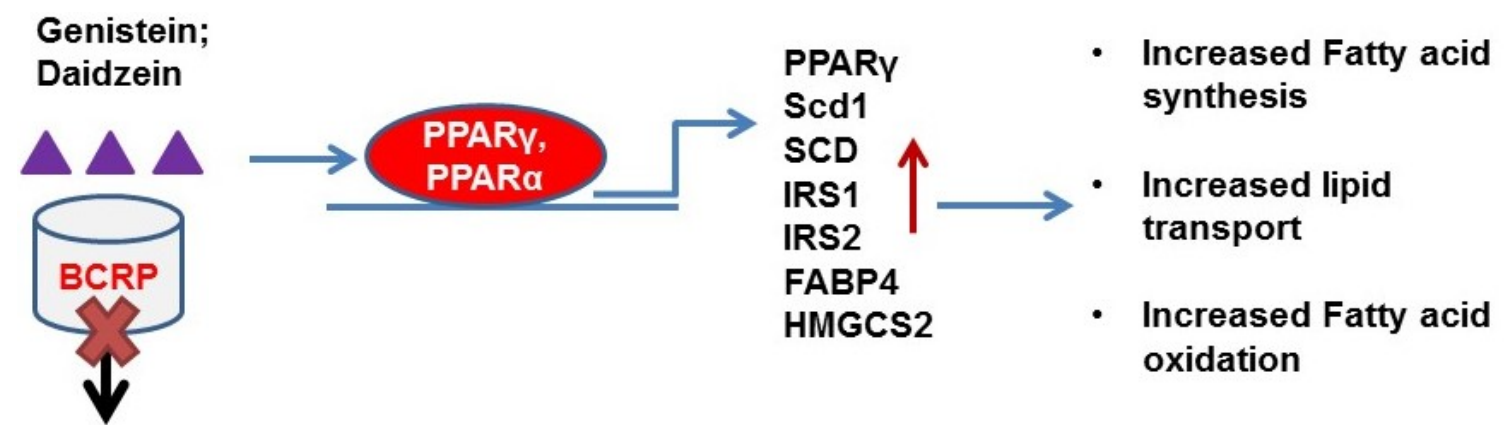

Figure 2-17. Possible explanation of activation of peroxisome proliferatoractivated receptor pathway in absence of Bcrp and/or Pgp

Notes: Bcrp substrate daidzein and also its metabolites were significantly increased in the plasma of $\mathrm{dKO}$ rats. Isoflavonoid and Bcrp substrates daidzein and genistein are known activator of PPAR $\alpha$ as well as PPAR $\gamma$ pathways. Gene expression analysis also observed gene expression changes possibly related to PPAR activation pathway, thus leading to alteration in fatty acid metabolic pathways. 


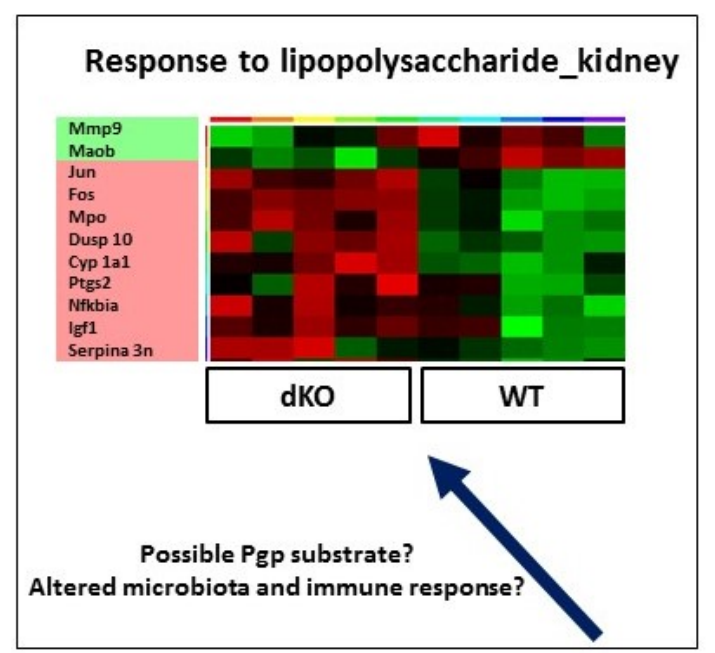

Response to oxidative stress pathway_kidney
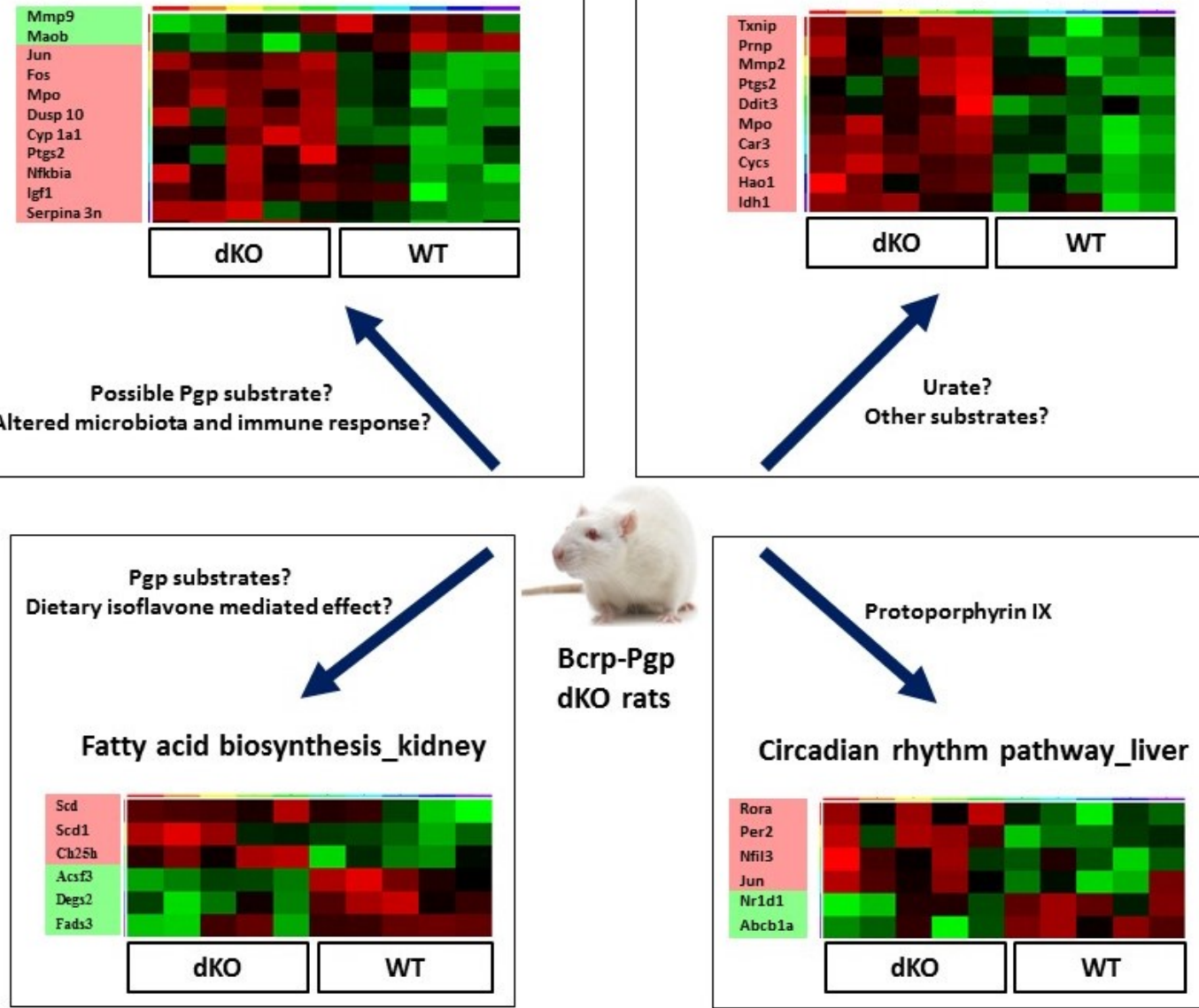

Figure 2-18. Possible biological pathways altered in the absence of Bcrp and Pgp in the dKO rats, based on both transcriptomic and metabolomic evidences 


\section{In silico prediction of BCRP and PGP substrate-like properties for the significantly altered metabolites in CSF and plasma of dKO rats}

Screening of all 169 metabolites significantly altered in CSF and plasma of dKO rats for their interaction with Bcrp and /or Pgp may not be a feasible option. Also, it is unlikely that all observed metabolic changes were due to the direct effect of Bcrp and/or Pgp transport deficiency. Therefore, we used an in silico prediction-based approach to identify metabolites that are more likely to be substrates of Bcrp and/or Pgp. A Bayesian machine learning model, built with compounds known to interact with Bcrp and Pgp, was used to score the metabolites based on their level of interaction (high score for higher interaction). Compared to the xenobiotics, many of these metabolites are smaller molecules, and might also have weaker interaction with the transporters, which is also reflected by their low interaction score. As described in the methods, Bcrp or Pgp Bayesian interaction score for each metabolite along with their molecular descriptors (molecular weight, molecular polar surface area, AlogP etc.) were used to predict the observed $\log 2$ (fold change) of the metabolites in CSF and plasma. Metabolites in the plasma, irrespective of their significance and direction of fold change, did not correlate well with either the Abcg2 or Pgp score along with other descriptors (data not shown). A significant correlation was observed for the metabolites that are significantly altered in the CSF of dKO compared WT rats (Figure 2-19A). In particular, metabolites that significantly increased by $>1.5$-fold in the CSF of dKO rats, correlated strongly with the ABCG2 score, $\log \mathrm{P}$ and molecular polar surface area of the metabolites (Figure 2-19B). Raw data for the significantly altered CSF metabolites that are used for this analysis is presented in Supplement_Chapter 2_Metabolites_Table S4.

\section{Discussion}

The goal of the current study is to understand the effect of Bcrp and Pgp efflux transporters on the rat metabolome, and elucidate their biological functions. A systemic level understanding of the impact of these transporters on metabolomic and transcriptomic profiles could be useful to identify their overall endogenous function, leading to a better understanding of long-term inhibition of these transporters by xenobiotics or dietary inhibitors, as well as elucidate effects of the well-known BCRP polymorphism [111] on population physiology and drug treatment outcome.

Bcrp and Pgp are abundant at the blood brain barrier (BBB), blood CSF barrier (BCSFB) and on enterocytes of small intestine, where they restrict brain permeability and oral absorption, respectively, of many known drugs and toxic chemicals [46, 149]. Absence of these transporters is expected to increase the concentration of their substrates in both blood and brain / CSF. Therefore, by comparing the CSF and plasma metabolomic profiles between WT and Bcrp-Pgp KO (dKO) rats, we aimed to identify putative endogenous substrates of Bcrp and/or Pgp and their impact on the systemic metabolome. 
A

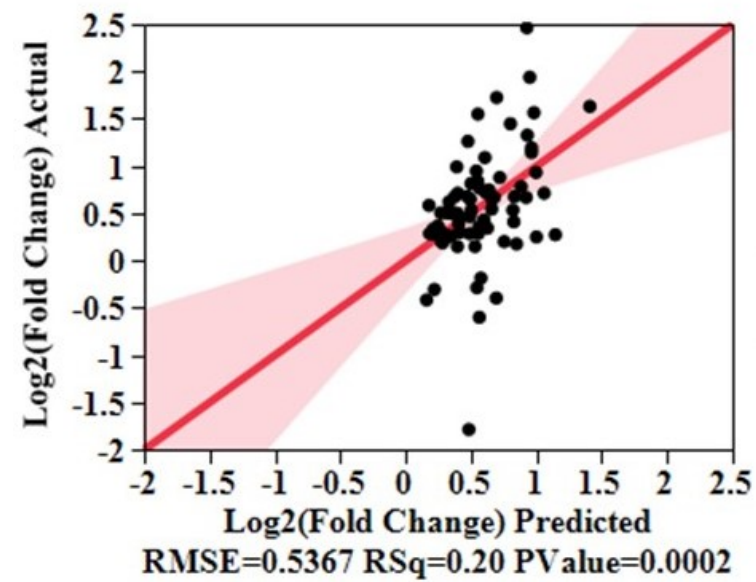

B

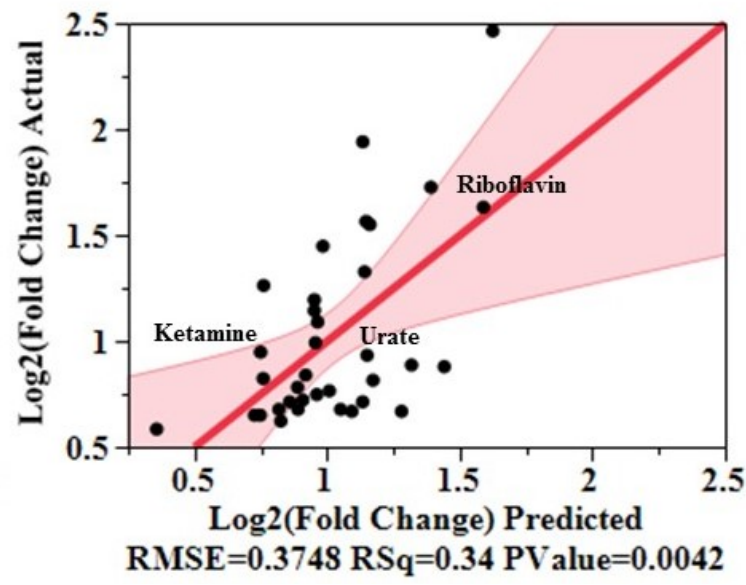

Figure 2-19. Metabolites with significantly higher abundance in dKO CSF compared to WT rats have structural properties for interaction with BCRP

Notes: ABCG2 or BCRP interaction score from a Bayesian model, molecular weight, molecular polar surface area and AlogP of each significantly altered metabolites (A) or only $>1.5$-fold significantly increased metabolites (B) in CSF were used in a predictive regression model to predict their fold change (dKO/WT) in CSF. Log2 (Fold change) actual vs. Log2 (Fold change) predicted compared using a standard least square method. Significance of the correlation computed at $\mathrm{p}<0.05$. 
We observed a significant difference in abundance of metabolites in CSF and plasma between WT and dKO rats. While $90 \%$ of the significant metabolites in CSF increased in $\mathrm{dKO}$ rats compared to WT, the plasma metabolome demonstrated $50 \%$ abundance of both increased and decreased metabolites. The majority of the altered plasma metabolomic signature was represented by the lipid metabolites. Specifically, long and medium chain fatty acids, dicarboxylate and polyunsaturated fatty acids were found to be significantly decreased in the plasma of dKO compared to WT rats. Similar to our observations, in a metabolomic study in mice after treatment with PPAR $\gamma$ agonist rosiglitazone, the authors reported a decrease in polyunsaturated fatty acids such as mead acid, oleic acid, phosphatidylethanolamine and phosphatidylcholine [197]. In addition, our transcriptomic analysis revealed significant alteration of genes related to the PPAR $\gamma$ pathway [197] such as uncoupling protein 1 (Ucp1, 1.4-fold increase in dKO kidney), fatty acid binding protein 4 (Fabp4, 2-fold increase in dKO kidney), leptin (Lep, 1.4-fold increase in $\mathrm{dKO}$ kidney) and lipoprotein lipase (lpl, 1.5-fold increase in $\mathrm{dKO}$ liver and 1.3-fold increase in $\mathrm{dKO}$ kidney). Of particular interest, we observed a 1.5-fold increase in PPAR $\gamma$ gene expression in both kidney and liver of $\mathrm{dKO}$ rats.

In a separate study, treatment with PPAR $\alpha$ agonist fenofibrate was reported to increase the acylcarnitine metabolites including propionyl-, isobutyryl-, 2-methylbutyryland isovalerylcarnitine in the urine of healthy volunteers [222]. A significant increase in these carnitine analogs was also observed in our dKO rat CSF metabolome signature (Table 2-3 and Supplement_Chapter 2_Metabolites_S1B). Interestingly, another PPAR $\alpha$ activity biomarker in mice, nicotinamide [222], was found to be significantly decreased in the CSF of the dKO rats. Furthermore, we observed a 0.56 -fold decrease in gene expression of carnitine palmitoyl transferase 1a (CPT1a) which is known to be altered due to PPAR $\alpha$ activity [222], in the liver of dKO rats. Alteration in amino acid and carnitine metabolites in CSF and plasma has been reported by another group of researchers in rats treated with pan-PPAR agonist tetradecylthioacetic acid [223]. In addition, a 5-fold increase in 2, 8-quinolinediol in the dKO rat plasma (Figure 2-4), is commensurate with previous findings in mice, where treatment of mice with PPAR $\alpha$ ligand Wy-14,643 significantly decreased urinary 2, 8-dihydroxyquinoline [224]. Therefore, taken together, our metabolomic and transcriptomic results suggest a possible induction of the PPAR $\alpha$ and PPAR $\gamma$ pathways in rats lacking Bcrp and Pgp transporters, possibly leading to the observed changes in fatty acid and amino acid metabolites (Table 2-1, Table 2-2, Table 2-3, Supplement_Chapter 2_Metabolites_Table S5 and S6). This implies that in the absence/inhibition of Bcrp and Pgp in enterocytes, substrates of these transporters, which are also PPAR $\alpha / \gamma$ agonists, such as dietary isoflavones genistein [225-229] and daidzein [230, 231] (was increased 2 fold in our dKO rat plasma, Figure 2-11), may be absorbed more, leading to higher PPAR $\alpha / \gamma$ activity in the system (Figure 2-17).

Another striking effect on the plasma lipid metabolome was the overall increase in lysolipids (Table 2-2). In a recently published report on identification of early biomarkers of lipopolysaccharide (LPS)-induced sepsis in rats, the authors reported an alteration in palmitoyl carnitine, acetyl-L-carnitine, propionyl-L-carnitine, branched chain amino acids, along with a significant increase in methionine, $\mathrm{N}$-acetyl methionine, 
methionine sulfoxide and lysolipids [232]. Our metabolomic data demonstrated an increase in methionine, $\mathrm{N}$-acetyl methionine and methionine sulfoxide in CSF

(Supplement_Chapter 2_Metabolites_Table S1B) and lysolipids in plasma (Table 2-2) of the dKO rats. In addition, transcriptomic analysis found 'response to lipopolysaccharide' as one of the top 10 significantly altered biological pathways in liver, kidney and brain of dKO rats (Table 2-11). LPS is a complex large glycolipid found on the outer membrane of Gram (-) bacteria and can act as an endotoxin. Gut microbiota can utilize LPS to cause low grade inflammation in the host and thereby associate with different pathogenesis of the host, such as obesity and cardiovascular risk [233]. Therefore, our findings strongly suggest that the absence of Pgp/Bcrp has affected the gut microbiota, and the altered microbiota, in turn, is influencing both the plasma and CSF metabolome in $\mathrm{dKO}$ rats. The decrease in the secondary bile acid metabolites, such as deoxycholate $(0.55$-fold, $0.1>\mathrm{p}>0.05)$, glycodeoxycholate $(0.1$ fold, $\mathrm{p}<0.01)$, taurodeoxycholate $(0.57$ fold, $0.1>\mathrm{p}>0.05)$ and tauroursodexycholate $(0.58$ fold, $0.1>\mathrm{p}>0.05$ ), which are majorly derived by the gut microbiota, in the plasma of $\mathrm{dKO}$ rats (Table 2-1), also indicates a possible change in the gut microbiome in the absence of Bcrp and Pgp transporters.

Additional evidence of altered gut microbiome, in the absence of Bcrp and Pgp, comes from the signatures of cresol sulfate and phenol sulfate metabolites. Both are tyrosine metabolite products of the gut microbiota [234]. While cresol sulfate was significantly decreased in plasma, phenol sulfate was increased in the CSF of dKO rats (Table 2-4). Phenol sulfate is produced by E. Coli species of bacteria, which have been reported to increase in abundance in mice lacking Mdrla (Pgp) [162, 235]. Thus, a dysregulation in bacterial abundance in the $\mathrm{dKO}$ rats, leading to altered production of metabolites, might have led to some of these observed metabolomic changes. Our results also show alteration in other known gut microbiota metabolites in the plasma of $\mathrm{dKO}$ rats, such as the tryptophan metabolite indolelactate (1.5-fold increase) and phenylalanine and the tyrosine metabolite phenylacetyleglutamine (0.46-fold increase) (Table 2-4). Some of these metabolites were also found to be significantly increased in the CSF of $\mathrm{dKO}$ rats (Table 2-4). While our findings indicate an alteration in gut microbiome metabolites in the absence of Bcrp and Pgp transporters in rats, it remains to be understood which altered microbiome metabolites are due to exclusive alteration in the gut microbiome itself, and/or due to a change in the efflux transporters for these metabolites.

Sulfated metabolites, many of which are of gut microbiota origin, were significantly increased in the CSF and plasma of dKO vs. WT rats (Table 2-5 and Table 2-6). Bcrp is known to be a broad-specificity phytoestrogen sulfate transporter [127]. Dietary isoflavones such as genistein and daidzein, as well as their sulfated metabolites are also known substrates of Bcrp [93, 225]. Although cresol sulfate has been shown to be a Bcrp substrate [83], a decrease in plasma abundance of cresol sulfate, which is a gut microbiota-generated metabolite, indicated a possible alteration in the gut microbiome, as discussed before. Many sulfated metabolites of gut microbiota origin are also uremic toxins [212] and are known to interact with Bcrp as well as MRP4 and OAT, e.g., indoxyl sulfate $[126,141]$. Thus, it is also possible to have functional inhibition of multiple efflux 
or uptake transporters, in the absence of Bcrp function, in $\mathrm{dKO}$ rat enterocytes, leading to alterations in sulfated metabolites. Accordingly, in CSF and plasma of dKO rats, we observed significant changes in p-cresol sulfate ( 0.5 -fold, decrease, plasma), pyrocatechol sulfate (catechol sulfate, 3.3-fold increase, CSF) and equol sulfate (1.6-fold increase, plasma), which are known to be gut microbiota-derived uremic toxins [212]. Among them, p-cresol sulfate and equol sulfate are already known Bcrp substrates [83, 127]. We also observed a 2.14-fold higher CSF/plasma ratio of pyrocatechol sulfate in the $\mathrm{dKO}$ rats compared to WT, implying a significant accumulation of this uremic toxin in the CSF of dKO rats. Hence, due to known affinity of Bcrp towards sulfated metabolites [121], we think pyrocatechol sulfate may be a putative endogenous Bcrp substrate. Another such metabolite, 2-aminophenol sulfate, was found to be 3-fold higher only in the CSF of dKO rats (Table 2-6). In addition, the CSF/plasma ratio of 2aminophenol sulfate was 1.9-fold higher in dKO vs WT rats (Supplement_Chapter 2_Metabolites_Table S2). Known as a uremic toxin [142], this metabolite could also be of interest as a putative Bcrp substrate.

As discussed before, these uremic toxins and sulfated metabolites can be substrates of multiple transporters and be part of the Remote Sensing and Signaling [142] mechanism. In a recently published report, comparing the plasma metabolomics of Oat3 knockout and WT mice, Bush K et.al observed a significant increase in catechol sulfate, O-methyl catechol sulfate and 2-aminophenol sulfate among many other metabolites in the plasma of Oat3 knockout mice [140]. Interestingly, we also observed a 3 fold increase in catechol sulfate and 2-aminophenol sulfate and a 5-fold increase in O-methyl catechol sulfate in the CSF of dKO compared to WT rats (Table 2-6), but no change in the expression level of OAT1/3 transporters in any tissue (Table 2-9). In addition, the $\mathrm{CSF} /$ plasma ratio was 2-fold higher for catechol and 2-aminophenol sulfate and 13-fold higher for O-methyl catechol sulfate in dKO vs WT rats (Supplement_Chapter 2_Metabolites_Table S2). While the increased concentration of catechol and 2aminophenol sulfate in the $\mathrm{dKO}$ rat plasma could be explained by inhibition of renal Oat3 or Oat1 transporters, their accumulation in the CSF of Bcrp and Pgp dKO rats cannot be solely explained by these primarily kidney transporters, raising the possibility that these metabolites are also substrates of Bcrp and/or Pgp. A recent observation that the association of methotrexate clearance variability with the liver transporter OATP1B1 polymorphism was primarily related to inhibition of renal methotrexate transporter OAT1/3 by endogenous metabolites of OATP1B1 [213], further strengthens the Remote Sensing and Signaling hypothesis.

Similar to previously published findings in single Pgp or Bcrp knockout rats [91], our transcriptomic analysis did not reveal a drastic compensatory effect in gene expression of uptake (Table 2-9) or efflux transporters (Table 2-8) in the dKO rats. However, the Remote Sensing and Signaling hypothesis, discussed above, can lead to alteration of many transporter activities. One significant observation was the reduction in Slc22a5 or the Octn2 transporter in kidney, a known carnitine transporter [202], which might contribute to the observed alterations in carnitine metabolites. Glucocorticoids are known substrates of Pgp [57]. However, a significant increase (2 fold) in Cyp $2 b 1$ gene expression, which is inducible by glucocorticoids [236] and also metabolizes the same, in 
the liver of $\mathrm{dKO}$ rats, might further explain the decrease in corticosterone levels in the plasma of dKO rats (Figure 2-16).

Due to the significant impact of Bcrp and Pgp transporters on the brain penetration of their substrates [50, 237], we think CSF might be a better matrix to identify putative Bcrp and/or Pgp endogenous substrates than plasma. When we used in silico Pgp and Bcrp scores, along with molecular descriptors (AlogP, molecular weight, polar surface area) to predict the log2(fold change) of metabolites observed in plasma and CSF, only significantly altered metabolites in CSF, and not plasma (data not shown), displayed a better correlation between predicted and observed data (Figure 2-19). Correlation was also found to be better with only significantly increased metabolites in the CSF of dKO rats (Figure 2-19B), thus implying that these metabolites in dKO rats possessed Bcrp substrate-like properties. In addition, Random Forest Analysis identified metabolites based on their importance in group separation in the CSF of dKO compared to WT rats (Figure 2-7), which included known Bcrp substrates riboflavin and urate in the CSF and only riboflavin in the plasma. Of note, our metabolomic analysis identified ketamine, the drug used for anesthetizing the rats, increased 2-fold in both CSF and plasma of dKO rats compared to WT. Further investigation revealed that ketamine oral bioavailability and ketamine-induced loss of righting reflex pharmacodynamic activity was significantly higher in Bcrp-Pgp dKO and Pgp KO mice, indicating ketamine is a dual substrate of Bcrp and Pgp [95]. In addition, Kaiser et al. recently reported that ketamine is a substrate of Pgp in vitro [98], further validating our hypothesis that metabolomic analysis could identify novel substrates of Bcrp and Pgp. Our observation that pesticide chlorothalonil metabolites 4-hydroxychlorothalonil was increased in the plasma of $\mathrm{dKO}$ rats, indicates possible risk of exposure to the pesticide in people lacking BCRP and/or PGP function. Lack of 4-hydroxychlorothalonil in CSF also indicates the involvement of other efflux mechanism at the BBB that prevents this metabolite from getting into CSF.

Among the known Bcrp substrates, urate's signature in the CSF was of particular interest. Urate was found to be significantly increased only in the CSF of dKO rats (Figure 2-11). In addition, the CSF/plasma ratio of urate was found to be 3-fold higher in the $\mathrm{dKO}$ compared to WT rats. While elevated serum urate is known to be positively correlated to gout, cardiovascular and renal diseases, it has been shown to be negatively correlated to Alzheimer's and Parkinson's diseases [238]. Bcrp is a low affinity and high capacity transporter of uric acid [54] and has also been associated with Alzheimer's disease [207]. Thus, altered uric acid in CSF of dKO rats may have varied physiological consequences. In addition, uric acid has been reported to be both antioxidant as well as causing oxidative stress [239, 240]. It has also been associated with causing endoplasmic reticulum stress leading to changes is fatty acid metabolism [241]. Similar findings were observed in our metabolomic analysis with a significant increase in methionine sulfoxide, a possible biomarker of oxidative stress [242] along with 'oxidative stress' as a significantly altered biological pathway in the transcriptomic analysis. Furthermore, uric acid is known to inhibit UMP synthase [243] that can reduce orotidine metabolism, which might explain the observed increase in orotidine in the CSF and plasma of dKO rats. Apart from urate and its downstream metabolite allantoin and allantoic acid, xanthosine, 
adenine containing- and guanine containing- purine metabolites were found to be significantly increased in the CSF of the dKO rats (Supplement_Chapter 2_Metabolites _ S6). Similarly, pyrimidine, specifically orotate and uracil containing, metabolites also significantly increased in the CSF of dKO rats. In an unpublished previous study in the lab, comparing the CSF of WT and Bcrp KO female rats $(n=12)$, we also observed increased xanthine along with urate and allantoin in the CSF of Bcrp KO compared to WT female rats. Thus, alteration in purine metabolite pathway might be related to the absence of Bcrp transporter. Bcrp is also known to have affinity for purine ring containing chemicals [221], raising the possibility that some of the purine pathway metabolites apart from urate could be putative Bcrp substrates.

Comparison of gene expression between WT and dKO rat tissues by microarray also identified 'circadian rhythm' pathway as a significantly altered biological pathway. This is particularly interesting since Bcrp substrate protoporphyrin IX is a porphyrin metabolite, and may influence the circadian rhythm pathway. Indeed, cobalt and zinc conjugated protoporphyrin IX (PPIX) have been shown to be a ligand activator of REV$\mathrm{ERB} \alpha$ / NR1D1, important for the circadian rhythm pathway [244]. In a previous experiment in our lab, we observed a 6-fold higher PPIX in the red blood cells (RBCs) of Bcrp KO female compared to WT female rats (data not shown). Thus, it is possible, PPIX is mediating the changes in the circadian pathway that we observed in our experiment. Evidence for this comes from the microarray analysis of the dKO vs WT kidney and liver that showed changes in expression of Ror $\alpha$, Per2 and Nr1d1 and ATF3, and the mouse phenome database showing abnormal sleep behavior in Bcrp knockout mice (http://www.mousephenotype.org/data/genes/ MGI:1347061\#section-associations). Bcrp gene expression in the mouse intestine was recently reported to be under the control of circadian clock-activating transcription factor 4 (ATF4) pathway, and authors showed a clear difference in sulfasalazine absorption when administered orally at Zeitgeber time 2 (ZT2) compared to 14 (ZT14) [245].

While our results indicate an effect of both Bcrp and Pgp transporters on the endogenous metabolome and biological pathways, further studies are required to dissect out the effect of the individual transporters. Due to lack of an exhaustive database and useful analytical methods for our dataset, we have used a knowledge-based integration of transcriptomic and metabolomic data. Our study design falls under a source-matched study design for metabolomic and transcriptomic studies [194] usually performed in animals, and the difficulty of analyzing the data lies in the integration of metabolomic and transcriptomic information originating from different matrices. In particular, the plasma metabolome can reflect the overall change from almost all highly perfused tissues. In this regard, while our data demonstrates an effect of Bcrp and/or Pgp transporters on the gut microbiome, further studies are needed for better understanding of this effect. Although our metabolomics comparison identified known Bcrp substrates, urate, pheophorbide $\mathrm{A}$, riboflavin in the $\mathrm{dKO}$ rat CSF and/or plasma, the metabolomic analysis did not identify PPIX in the rat plasma/CSF. PPIX is a high affinity substrate of Bcrp, that is otherwise trapped in RBCs [246], suggesting that we could miss some hydrophobic substrates trapped in other cells that highly express Bcrp. 
In conclusion, our results demonstrate a significant impact of the two efflux transporters Pgp and Bcrp on the systemic metabolome in rats and have increased our understanding of the endogenous function of these transporters. To our knowledge, this is the first study towards understanding the systemic level impact of efflux transporters. We have also shown that CSF and plasma metabolome can be independent from each other and the effect of Bcrp and/or Pgp transporters in these two compartments can be very different. Finally, our results demonstrate the importance for considering the Remote Sensing and Signaling hypothesis for chronic use of transporter inhibitors, and might throw some light on pharmacokinetic/ pharmacodynamic variability of many drugs that are substrates or inhibitors of these transporters. 


\title{
CHAPTER 3. KETAMINE PHARMACOKINETICS AND PHARMACODYNAMICS ARE ALTERED BY PGP AND BCRP EFFLUX TRANSPORTERS IN MICE*
}

\begin{abstract}
Introduction
Ketamine is a clinically useful anesthetic that is used either alone or in combination with other drugs in different surgeries [247]. It is also useful for the management of neuropathic and postoperative pain [248, 249]. It has been reported recently that ketamine has long-lasting antidepressant activity at sub-anesthetic doses [250]. But clinical utility of ketamine is affected by its recreational abuse, which often reported to cause kidney pathology, ulcerative cystitis [251] and bladder toxicity [252] in chronic ketamine abusers.
\end{abstract}

Pharmacologically, ketamine is a N-methyl-D-aspartate (NMDA) receptor antagonist that binds to the phencyclidine binding pocket of the receptor [253] to elicit anesthesia. However, ketamine is also known to interact with other pharmacological targets [247]. For example, ketamine was shown to have rapid onset antidepressant activity following metabolism to $(2 \mathrm{R}, 6 \mathrm{R})$-hydroxynorketamine that activates $\alpha$-amino-3hydroxy-5-methyl-4-isoxazole propionic acid receptors (AMPARs) [274].

Ketamine pharmacokinetics (PK) in humans are well-described by a two or three compartment PK model $[254,255]$ with a high volume of distribution, high clearance and low plasma protein binding [253]. Ketamine is metabolized primarily by CYP2B6 and CYP3A4 to form norketamine, which is further metabolized to hydroxynorketamine and its glucuronide [256]. Ketamine has a low oral bioavailability that can be partially explained by its high metabolism observed by the oral route compared to intravenous or intramuscular routes [255, 257]. In addition, modifications in ketamine metabolism have been reported to cause potential drug-drug interactions [79].

Breast cancer resistant protein (Bcrp/Abcg2) and P-glycoprotein (Pgp/Abcb1a) are membrane-bound efflux transporters that are present in a wide variety of tissues such as blood-brain barrier and blood-cerebrospinal fluid barrier in the brain, intestinal epithelial cells, and bile canalicular membrane and kidney tubules. These transporters recognize a wide variety of chemicals as their substrates and reduce substrate systemic exposure by decreasing absorption (intestine) and increasing elimination (liver and kidney). Pgp and BCRP expression at the BBB and BCSFB decreases substrate movement from blood to brain and to CSF, respectively. Thus, inhibition of Bcrp and / or

\footnotetext{
*Reprinted with permission of the American Society for Pharmacology and Experimental Therapeutics. All rights reserved. S Ganguly, JC Panetta, JK Roberts, and EG Schuetz. Ketamine Pharmacokinetics and Pharmacodynamics Are Altered by Pgp and Bcrp Efflux Transporters in Mice, Drug Metabolism and Dispos (2018), DOI:

https://doi.org/10.1124/dmd.117.078360
} 
Pgp can lead to systemic overexposure of their substrates and cause toxicity. A wide variety of drugs as well as chemicals from food or drinks are known to inhibit these transporters, and can therefore lead to potential drug-drug or drug-food interactions [71, 258, 259]. Moreover, the BCRP Q141K inactivating allele is common in some populations with as many as $6 \%$ of Asians homozygous for this loss of function allele [111].

Screening drugs in development for Bcrp and Pgp transport and inhibition capability is one of the FDA required drug approval criteria [31, 71] because of the large number of Bcrp and Pgp substrates and inhibitors, and the potential for some chemicals to be dual substrates for both transporters. Some in vivo studies in rodents as well as in humans have suggested a potential interaction of ketamine with Bcrp and/or Pgp [260, 261]. Given the clinical and recreational use of ketamine, and the significant interest in ketamine as an effective anti-depressant, we sought to identify if ketamine is a substrate of Bcrp and/or Pgp. Using mice with and without these two transporters, we report for the first time that ketamine is an apparent dual Pgp/Bcrp substrate whose pharmacokinetic and pharmacodynamic properties are affected by both Pgp and Bcrp.

\section{Materials and Methods}

\section{Drugs and chemicals}

Ketamine hydrochloride injection USP $(100 \mathrm{mg} / \mathrm{ml})$ was purchased from JHP Pharmaceuticals (Rochester, MI), elacridar was purchased from Astatech Inc. (Bristol, PA), Hydroxypropylmethylcellulose (HPMC) and Tween 80 were purchased from Sigma-Aldrich (St Louis, MO), Promega Reporter Lysis buffer (5X), PierceTM BCA protein assay reagent (A and B), bupropion, acetonitrile, formic acid and ammonium hydroxide were purchased from Fisher Scientific (Pittsburgh, PA). 3H-ketamine was obtained from American Radiolabeled Chemicals (St.Louis, MO).

\section{Animals and cell lines}

Wild-type (WT) and Bcrp/Pgp (Abcg2-Abcb1a) double-knockout (dKO) male Sprague-Dawley rats, weighing $200-250 \mathrm{~g}$, were obtained from SAGE® Labs (Horizon Discovery - St Louis, MO). Male Bcrp (Abcg2) KO, Pgp (Abcb1a/b) KO, Pgp/Bcrp (Abcb1a/1b/ Abcg2) double KO (dKO), and Wild-type Friend virus B (FVB) mice were obtained from Taconic farms (Germantown, NY). Rats used in the experiments were 12 weeks old and mice were 10-16 weeks old. All animals were provided water and food ad libitum. Ketamine hydrochloride stock solution $(100 \mathrm{mg} / \mathrm{ml})$ was diluted with filtered autoclaved water to the appropriate dilution for dosing. Elacridar $(10 \mathrm{mg} / \mathrm{ml})$ was formulated by suspending elacridar in a solution of $0.5 \%$ Hydroxypropylmethylcellulose (HPMC) with 1\% Tween 80. A 1.5 inch 20 GA feeding needle (Fisher Scientific Co., Pittsburgh, PA) was used to perform oral dosing. The Institutional Animal Care and Use 
Committee of St. Jude Children's Research Hospital in accordance with the U.S. National Institutes of Health guidelines approved all experimental procedures.

MDCKII parental cells and derivative cells stably overexpressing both human PGP and BCRP were kindly provided by Dr. Alfred H. Schinkel's lab (The Netherlands Cancer Institute, Amsterdam) and cultured as described [262].

\section{Drug formulations for animal experiments}

Ketamine hydrochloride stock solution $(100 \mathrm{mg} / \mathrm{ml})$ was diluted with filtered autoclaved water to appropriate dilution for oral (PO) or intraperitoneal (IP) dosing. Elacridar (solution) formulation was prepared by suspending the appropriate amount of elacridar in $0.5 \%$ Hydroxypropylmethylcellulose (HPMC) with $1 \%$ Tween 80 solution to achieve a final concentration of $10 \mathrm{mg} / \mathrm{ml}$.

\section{Estimation of ketamine exposure in rat CSF and plasma}

Male WT and Pgp/Bcrp dKO rats ( $\mathrm{n}=8$ per group) were anesthetized with intraperitoneal ketamine $(100 \mathrm{mg} / \mathrm{kg})$ and xylazine $(10 \mathrm{mg} / \mathrm{kg})$ formulated in water, at a dose volume of $10 \mathrm{ml} / \mathrm{kg}$ of body weight. Cerebrospinal fluid (CSF) was collected from the cisterna magna immediately after the anesthesia was achieved ( $\sim$ minutes), followed by blood collection by cardiac puncture. Blood samples were centrifuged and plasma separated from the blood. Both plasma and CSF samples were immediately frozen on dry ice, stored at $-800 \mathrm{C}$, and shipped to Metabolon ${ }^{\circledR}$ (Research Triangle Park, NC) for metabolomic analysis. LC-MS/MS sample analysis, data normalization and analysis were performed at Metabolon $\AA$ as described in other reports [186]. Normalized ketamine peak area intensity values were compared between the WT and dKO rats in both CSF and Plasma.

\section{Pharmacokinetic study of ketamine in WT, Bcrp KO, Pgp KO and dKO mice after oral and intraperitoneal dosing at $100 \mathrm{mg} / \mathrm{kg}$}

A serum pharmacokinetic study was conducted following ketamine intraperitoneal and oral dosing (IP and PO PK) of WT, Bcrp KO, Pgp KO and dKO mice at $100 \mathrm{mg} / \mathrm{kg}$ dose of ketamine. For IP PK, six mice from each genotype were divided into two groups of three mice each. After the animals were dosed IP, blood was collected from one group of mice at 2, 10 and 40 minutes, and from another group of mice at 5, 20 and 60 minutes. At each time point, $50 \mu \mathrm{L}$ blood was withdrawn from each mouse from the saphenous vein into microvette capillary blood collection tubes (Sarstedt, obtained from Fisher Scientific, Pittsburgh, PA). Samples were kept at room temperature and were centrifuged at 10,000 rpm for 5 minutes to obtain serum, which was immediately separated and stored at $-80^{\circ} \mathrm{C}$ until further analysis. For the PO PK study, six mice from each genotype were separated into two groups (three mice in each group) and after oral 
dosing blood samples were collected at alternative time points from each group similar to the IP study. After a two-week washout period, the same oral study was repeated by altering the groups and time points such that all time-points were collected from each mouse. The two PO PK data sets were first analyzed separately and compared to identify any significant inter-day variation, and, after confirming no inter-day difference in PK profile, the data from the two collection dates were merged to obtain complete PK profiles for each mouse.

\section{Determination of ketamine concentration in mouse serum by HPLC/UV}

Serum samples from the intraperitoneal ketamine PK studies in mice were treated with chilled acetonitrile (1:5); samples were vortexed and centrifuged for 15 minutes at $4000 \mathrm{rpm}$ to precipitate the protein and extract ketamine. After centrifugation, the supernatant was collected and further centrifuged for sample cleaning. The resultant final supernatant was then diluted 1:1 with water: acetonitrile $(1: 1)$ and a $10 \mu \mathrm{L}$ sample was injected onto the HPLC for further analysis. For PO PK, a 1:2.5 initial dilution with chilled acetonitrile was used, followed by centrifugation and protein precipitation and recentrifugation of the supernatant for sample cleaning. The final supernatant was injected onto the HPLC system (Shimadzu Prominence, Kyoto, Japan) consisting of a LC-20AD binary high pressure gradient pump, SIL-20ACHT auto sampler, and SPD-20AV UVdetector. Bupropion $(100 \mu \mathrm{g} / \mathrm{mL})$ was added as an internal standard to all the samples prior to extraction and was extracted along with ketamine. Chromatographic separation of ketamine along with the internal standard was achieved using Hypersil ODS C18 column (150X4 mm; $5 \mu \mathrm{m}$ particle size) fitted with Hypersil ODS (C18) Javelin Guard Column (10X4 mm; $5 \mu \mathrm{m}$ particle size) and a gradient elution method with a run time of 10 minutes. The mobile phase for elution was 60:40 ammonium formate buffer $(20 \mathrm{mM})$ : acetonitrile [263] and the gradient used was: 0 to 3 minutes $40 \%$ acetonitrile, 3 to 8 minutes $60 \%$ acetonitrile, 8 to 9 minutes $40 \%$ acetonitrile, and the run stopped at 10 minutes. Retention times for ketamine and bupropion were 5.2 and 7.1 minutes, respectively. The ketamine peak was identified and absorbance was measured at $210 \mathrm{~nm}$ [264]. Analyte/IS height ratio was used for preparation of calibration and measurement of unknown concentrations. Extraction efficiency was $>90 \%$ throughout the analysis. The calibration range for IP PK and PO PK analysis were $0.39 \mu \mathrm{g} / \mathrm{ml}-200 \mu \mathrm{g} / \mathrm{ml}$ and 0.19 $\mu \mathrm{g} / \mathrm{ml}-100 \mu \mathrm{g} / \mathrm{ml}$, respectively.

\section{Measurement of ketamine brain/serum ratio following IP ketamine}

Male FVB WT, Pgp KO and Pgp/Bcrp dKO mice were dosed with IP ketamine $(100 \mathrm{mg} / \mathrm{kg})+1 \mu \mathrm{Ci} 3 \mathrm{H}$-ketamine tracer $1 \mathrm{hr}$ before and again $15 \mathrm{~min}$ before sacrifice. Mice were euthanized by $\mathrm{CO}_{2}$ inhalation, blood was collected and serum separated, and brain tissue was weighed and mixed with an equivalent volume of phosphate buffer saline (PBS) and homogenized. Brain homogenate and serum were mixed with scintillation fluid and total radioactivity determined by liquid scintillation counting. The 
${ }^{3} \mathrm{H}$-ketamine brain-to-serum ratio is dpm/gram brain tissue homogenate divided by $\mathrm{dpm} / \mathrm{ml}$ of serum.

In another similar study, FVB male mice (WT, Pgp KO and Pgp/Bcrp dKO) were sacrificed 15 minutes after dosing $100 \mathrm{mg} / \mathrm{kg}$ ketamine intraperitoneally. Brain and blood were collected from each mouse after sacrifice. Brain tissues were weighed, homogenized with $20 \mathrm{mM}$ ammonium formate buffer, and $9 \mu \mathrm{L}$ brain tissue homogenate $+1 \mu \mathrm{L}$ IS was extracted using chilled acetonitrile. Supernate from the extracted samples were mixed with equal volume of acetonitrile/water (1:1) and injected in HPLC-UV for measurement of ketamine against standards prepared similarly from untreated mouse brain homogenate. Serum was separated from blood samples and ketamine concentration was measured by HPLC-UV based method as described previously. Brain/ serum ratio was obtained by dividing weight normalized brain tissue concentration with the serum concentration of ketamine.

\section{Pharmacokinetic data analysis and interpretation}

Non-compartment analysis. Initial analysis of both IP and PO PK study data was performed by a non-compartment approach using PK package on $\mathrm{R}$ statistical software [265] to determine whether there were significant differences in ketamine serum exposure between WT and Bcrp KO, Pgp KO and dKO mice. A serial sacrifice design was assumed to compute the area under time - concentration curve extrapolated to infinity (AUCinf) and the associated variability as described [265].

Nonlinear mixed effect modeling. Upon identification of significant exposure difference between genotypes in the PO PK study by non-compartment analysis, the PO and IP PK data were combined and analyzed with nonlinear mixed effect modeling implemented in Monolix 4.3.3 (Lixoft, France) using a stochastic approximation expectation maximization algorithm [266] combined with a Markov chain Monte Carlo procedure. Population analysis of our PK data did not find any difference in ketamine serum PK between WT and Bcrp KO mice and thus, these two genotypes were combined in the final model. Out of 144 data points in the PO PK study, there were four concentrations that were below the lowest calibration standard concentration $(0.19$ $\mu \mathrm{g} / \mathrm{mL}$ ) and these were removed from the analysis. One- and two-compartment models were evaluated. The goodness-of-fit-criteria and minimization of Akaike information criterion were used to select the base model. The effect of over-parameterization was also assessed using the condition number and Bayesian information criterion. Covariate analysis included the effect of genotype as a categorical covariate following an exponential model. The effect of genotype on individual parameters was determined by a forward addition process with a decrease in OFV $\geq 3.84$ considered significant at $p=$ 0.05 for one degree of freedom, based on $\mathrm{x} 2$ (chi-square) distribution. Variability in observed data from the model predicted population mean data is considered to be due to between-subject variability (BSV) and residual unexplained variability (RUV). The following equation was used to describe BSV, 


$$
\mathrm{Pi}=\theta \text { pop } \mathrm{x} \exp (\eta p o p)
$$

where, $\mathrm{Pi} \neg=\mathrm{PK}$ parameter of the ith individual; $\theta \mathrm{pop}=$ population mean for $\mathrm{P} ; \eta=$ normally distributed between-subject random effect with a mean of zero and variance of $\omega 2$. Constant, proportional and combined constant and proportional error models were tested for RUV, and the final model used the proportional error model that follows the following equation,

$$
Y i j=\hat{Y} i j+Y i j * \varepsilon
$$

where $\mathrm{Yij}=$ observed concentration of $\mathrm{ith}$ individual at time $\mathrm{j} ; \hat{\mathrm{Y}} \mathrm{ij}=$ individual predicted concentration and $\varepsilon$ is the proportional error that is normally distributed with a mean of zero and variance of $\sigma 2$.

Diagnostic plots were used for the final assessment of the model. Correlation between the observed and model predicted concentrations were evaluated and used as screening criteria for model selection. Population weighted residuals or individual weighted residuals were plotted against time and population or individual predicted ketamine concentration. Normalized prediction distribution error plotted against time and population predicted ketamine concentration was also evaluated for model misspecification assessment. Apart from the diagnostic plots, final model selection also included comparison of objective function values and variability associated with parameter estimates.

\section{Comparison of ketamine-induced duration of Loss of Righting Reflex (dLORR) between WT, Berp KO, Pgp KO and dKO mice}

Ketamine-induced dLORR was studied as described [267]. Briefly, animals were administered ketamine $(50,100$ or $200 \mathrm{mg} / \mathrm{kg}$ ) intraperitoneally or by oral gavage, and after four minutes waiting time (to reduce manual stimulation), mice were placed on their back. Loss of righting reflex was defined to have taken place when the mouse could not right itself for at least 20 seconds. The dLORR was then measured until the mouse woke up spontaneously and the dLORR was compared between WT, Bcrp KO, Pgp KO and dKO mice. In a separate study, WT mice were orally administered elacridar (ECD) (100 $\mathrm{mg} / \mathrm{kg}$ ) or vehicle 1.5 hours prior to IP administration of 50 or $100 \mathrm{mg} / \mathrm{kg}$ ketamine and dLORR was measured as described above.

\section{Intracellular uptake of radiolabeled ketamine in MDCKII cells overexpressing human PGP and BCRP}

Madine-Darby canine kidney II (MDCKII) cells +/- BCRP and PGP were plated at $0.2 * 10^{6}$ cells/ well in a 24 well plate. The next day media was removed and cells were incubated with $3 \mathrm{H}$-ketamine at $37^{\circ} \mathrm{C}$. At the selected time points, cells were washed with chilled PBS, incubated $1 \mathrm{hr}$ with lysis buffer, cell lysates collected, and the intracellular 
concentration of ${ }^{3} \mathrm{H}$-ketamine and protein concentration determined. Protein concentration was determined in the lysate by BCA protein assay. Results were expressed as pmole of ketamine per milligram of protein lysate. First, we determined the time to reach an equilibrium of intracellular uptake by incubating both WT and transfected cells with $0.5 \mu \mathrm{M}{ }^{3} \mathrm{H}$-ketamine for one hour and measuring the intracellular radioactivity at 2 , $5,15,30$ and 45 minutes. In a separate assay, the concentration-dependent uptake of ${ }^{3} \mathrm{H}-$ ketamine up to the time of equilibrium was determined in MDCKII and BCRP-PGPMDCKII cells by incubating the cells with different concentrations of ${ }^{3} \mathrm{H}$-ketamine until they reached equilibrium and then measuring intracellular concentration of ${ }^{3} \mathrm{H}-\mathrm{ketamine}$. The intracellular ketamine concentration was calculated and compared between the MDCKII and BCRP-PGP-MDCKII cells.

\section{Statistical analysis}

GraphPad QuickCalcs was used to perform Grubb's outlier test to remove outlier data from the analysis. Statistical analysis of significance for CSF and plasma ketamine exposure data in rats, all pharmacodynamic (dLORR) data, in vitro cell uptake study data, and non-compartment analysis were performed in GraphPad Prism software version 5.02. Ketamine's CSF and plasma peak intensity in the dKO rats and WT rats was compared using the unpaired t-test with Welch's correction. All dLORR study data was analyzed using Mann-Whitney $\mathrm{U}$ test. Intracellular uptake study results were analyzed using an unpaired t-test. The non-compartment-determined AUCinf was analyzed using a one-way ANOVA with Newman-Keuls Multiple Comparison Test. Significance for all analyses was equal to $\mathrm{p}<0.05$.

\section{Results}

\section{Deletion of both Bcrp and Pgp results in greater ketamine exposure in the CSF and plasma of dKO rats}

We performed a metabolomic comparison analysis of the CSF and plasma from WT and Bcrp-Pgp dKO rats anesthetized with a cocktail of ketamine-xylazine. A full report of the metabolomics results will be published elsewhere. Ketamine levels (measured by LC-MS/MS) were found to be significantly higher in the CSF (1.74 times) and plasma (1.93 times) of dKO compared to WT rats (Figure 3-1). These results suggested that ketamine might be a substrate of Bcrp and/or Pgp. The metabolomic analysis did not identify the presence of xylazine in either the rat CSF or plasma from either WT or Pgp/Bcrp KO rats. 


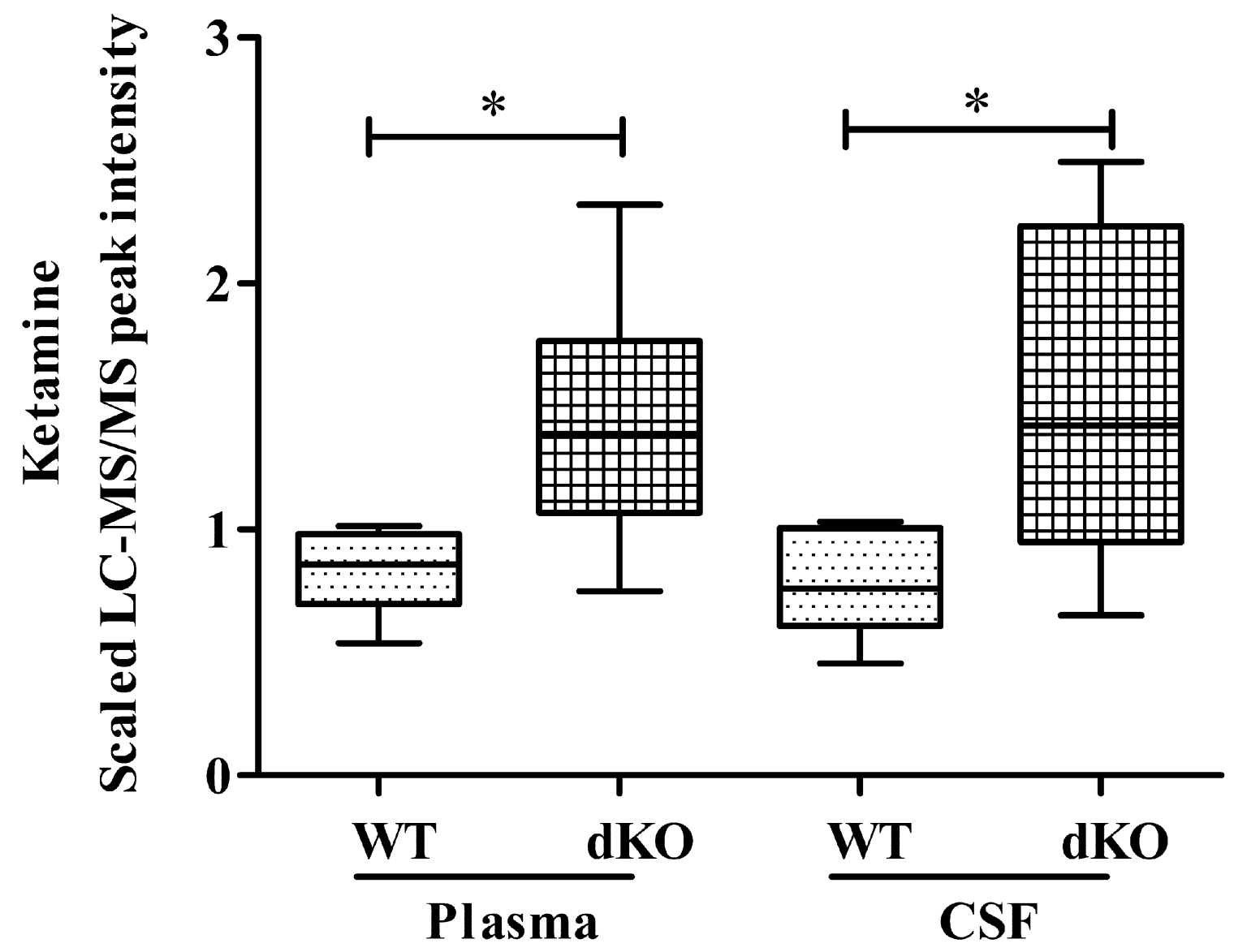

Figure 3-1. Elevated ketamine CSF and plasma levels in Pgp/Bcrp dKO vs. WT rats

Notes: Ketamine levels in male Sprague Dawley rats after IP administration of 100 $\mathrm{mg} / \mathrm{kg}$ ketamine. Data is plotted as median LC-MS/MS peak intensity \pm range $(\mathrm{n}=8 /$ genotype). The plasma and CSF median peak intensity in $\mathrm{dKO}$ rats was compared to the corresponding median peak intensity from WT rats using the unpaired t-test with Welch's correction. ${ }^{*} \mathrm{p}<0.05$ 


\section{Ketamine oral (PO) serum exposure is higher in mice lacking Bcrp and Pgp}

To determine if Bcrp and Pgp can affect the PK of ketamine, we collected serum following a $100 \mathrm{mg} / \mathrm{kg}$ IP dosing to WT, Bcrp KO, Pgp KO, and dKO mice. Serum concentrations of ketamine at the selected time points were found to be similar betweenall four genotypes (Figure 3-2A). Ketamine serum exposure, as measured by area under time - concentration curve ( $\mathrm{AUC}_{\text {inf }}$ ) by a non-compartment analysis $(\mathrm{NCA})$, was also found to be similar between all the genotypes (Figure 3-2B).

Since Bcrp and Pgp have a higher impact on their substrates serum/ plasma exposure after oral compared to IP dosing [268], we collected serum following a 100 $\mathrm{mg} / \mathrm{kg}$ oral dose of ketamine in WT, Bcrp KO, Pgp KO and dKO mice. Ketamine serum concentrations were significantly higher in the $\mathrm{dKO}$ mice compared to the WT mice (Figure 3-2C). Ketamine serum exposures ( $\mathrm{AUC}_{\mathrm{inf}}$ ), calculated by the non-compartment analysis method, were found to be significantly higher in dKO (1.96-fold) and Pgp KO (1.52-fold) mice and slightly higher in Bcrp KO (1.20-fold) mice compared to the WT

mice (Figure 3-2D). Ketamine serum concentration at the 60 minute time point could be detected in only two out of six WT mice, but in all of the Bcrp KO, Pgp KO and dKO mice. Thus, the last time point for observed concentration is different between WT and other knockout mice. Hence, $\mathrm{AUC}_{\mathrm{inf}}$ was used to compare the exposure between all genotypes. Both Pgp KO as well as Bcrp KO showed higher concentrations of ketamine than WT mice at earlier time points, indicating a possible alteration in absorption, and possibly driving the observed difference in AUCinf with the WT mice. Detailed noncompartment analysis parameters for both IP and PO PK studies are listed in Table 3-1. While the non-compartment analysis allowed comparison of concentration-time curve properties, there was no assumption of the compartment, and no information about which PK parameters could be causing the difference in $\mathrm{AUC}_{\text {inf, }}$, although there was the suggestion of an alteration in bioavailability because dividing the oral $\mathrm{AUC}_{\mathrm{inf}}$ by the IP $\mathrm{AUC}_{\text {inf }}$ gave an $(\mathrm{F})$ for the mice that was greatest in the $\mathrm{dKO}(\sim 27 \%)>\operatorname{Pgp} \mathrm{KO}(\sim 24 \%)$ $>$ Bcrp KO $(\sim 17 \%) \sim=$ WT $(\sim 14 \%)$.

\section{Population pharmacokinetic analysis demonstrates an increase in ketamine oral bioavailability in Pgp/Bcrp dKO and Pgp KO mice compared to WT and Bcrp KO mice}

To better understand the impact of Bcrp and Pgp mediated efflux on PK properties of ketamine, we combined both PO and IP PK data in a simultaneous model using a population pharmacokinetic approach. This model also assumed that bioavailability after IP dosing was equal to one. The final model was a one-compartment distribution with zero order absorption for $\mathrm{PO}$ and first-order absorption for the IP dosing and first-order elimination from the central compartment (Figure 3-3). The one compartment model was sufficient to describe these data (Figure 3-4, Figure 3-5 and Figure 3-6). The final parameters of population analysis are shown in Table 3-2. Transporter genotypes had no effect on clearance or volume of distribution. The small difference in ketamine PK between Bcrp KO versus WT mice observed by non- 

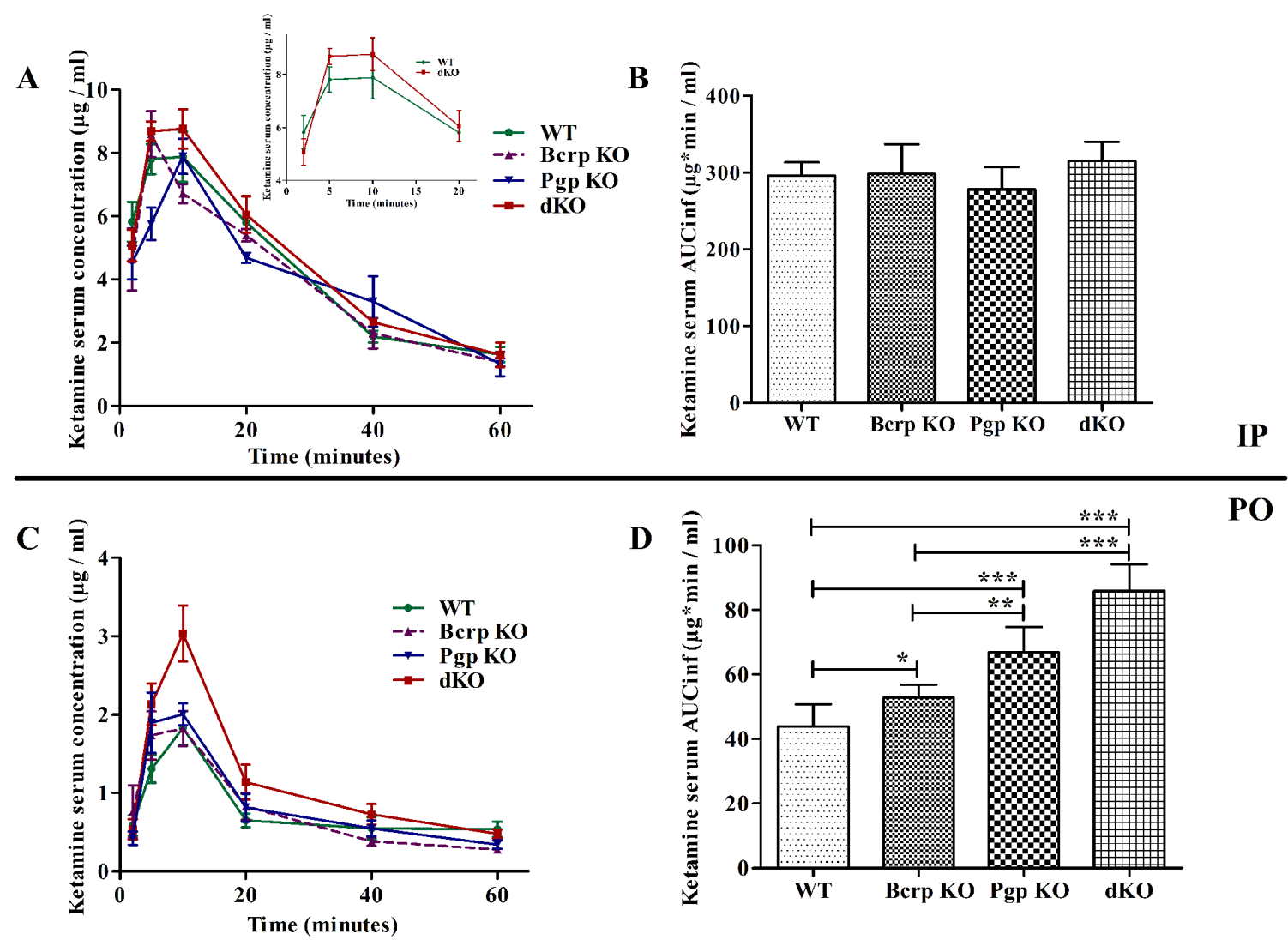

Figure 3-2. Ketamine serum exposure in Pgp/Bcrp dKO mice is significantly higher than in WT mice after oral dosing

Notes: (A) \& (C): Mean ketamine plasma concentrations \pm SE over time curves after IP ( $\mathrm{n}=3 /$ genotype) and oral (n=6/genotype) ketamine dosing, respectively of FVB WT, Pgp $\mathrm{KO}, \mathrm{Bcrp} \mathrm{KO}$ and dKO mice. (B) \& (D): Ketamine plasma $\mathrm{AUC}_{\mathrm{inf}} \pm \mathrm{SE}$ following IP and oral dosing of FVB WT, Pgp KO, Bcrp KO and dKO mice. The AUC was calculated by non-compartment analysis using the PK package on R. One way ANOVA with NewmanKeuls Multiple Comparison Test was used to compare $\mathrm{AUC}_{\text {inf. }}{ }^{*} \mathrm{p}<0.05,{ }^{* *} \mathrm{p}<0.01$, $* * * \mathrm{p}<0.001$. 
Table 3-1. Noncompartment analysis (NCA) parameters for ketamine IP and PO $\mathrm{PK}$ at $100 \mathrm{mg} / \mathrm{kg}$

\begin{tabular}{|c|c|c|c|c|c|}
\hline $\begin{array}{l}\text { Dosing } \\
\text { Route }\end{array}$ & $\begin{array}{l}\text { Parameters } \\
\text { (units) }\end{array}$ & WT & Bcrp KO & Pgp KO & $\begin{array}{c}\text { Double } \\
\text { KO }\end{array}$ \\
\hline \multirow{4}{*}{$\begin{array}{l}\text { IP PK } \\
(100 \\
\mathrm{mg} / \mathrm{kg})\end{array}$} & $\mathrm{t}_{1 / 2}(\operatorname{mins})$ & $22.93 \pm$ & $27.75 \pm$ & $22.72 \pm$ & $21.89 \pm$ \\
\hline & $\mathrm{T}_{\max }(\operatorname{mins})$ & 10.00 & 5.00 & 10.00 & 10.00 \\
\hline & $\begin{array}{l}\mathrm{C}_{\max } \\
(\mu \mathrm{g} / \mathrm{mL})\end{array}$ & $\begin{array}{c}7.88 \pm \\
1.38\end{array}$ & $8.60 \pm 1.03$ & $\begin{array}{c}7.90 \pm \\
0.98\end{array}$ & $\begin{array}{c}8.77 \pm \\
1.08\end{array}$ \\
\hline & $\begin{array}{l}\mathrm{AUC}_{\mathrm{inf}} \\
(\mu \mathrm{g} * \mathrm{hr} / \mathrm{mL})\end{array}$ & $\begin{array}{c}296.61 \pm \\
17.07\end{array}$ & $\begin{array}{c}298.4 \pm \\
38.43\end{array}$ & $\begin{array}{c}278.33 \pm \\
29.1\end{array}$ & $\begin{array}{c}315.15 \pm \\
25.38\end{array}$ \\
\hline \multirow{4}{*}{$\begin{array}{l}\text { PO PK } \\
(100 \\
\mathrm{mg} / \mathrm{kg})\end{array}$} & $\mathrm{t}_{1 / 2}(\mathrm{mins})$ & $\begin{array}{c}17.86 \pm \\
4.23\end{array}$ & $\begin{array}{c}22.26 \pm \\
4.07\end{array}$ & $\begin{array}{c}30.85 \pm \\
9.54\end{array}$ & $\begin{array}{c}26.56 \pm \\
6.25\end{array}$ \\
\hline & $\mathrm{T}_{\max }(\operatorname{mins})$ & 10.00 & 10.00 & 10.00 & 10.00 \\
\hline & $\begin{array}{l}\mathrm{C}_{\max } \\
(\mu \mathrm{g} / \mathrm{mL})\end{array}$ & $\begin{array}{c}1.83 \pm \\
0.52\end{array}$ & $1.82 \pm 0.54$ & $\begin{array}{c}2.0 \pm \\
0.34\end{array}$ & $\begin{array}{l}3.03 \pm \\
0.87^{*}\end{array}$ \\
\hline & $\begin{array}{l}\mathrm{AUC}_{\mathrm{inf}} \\
\left(\mu \mathrm{g}^{*} \mathrm{hr} / \mathrm{mL}\right)\end{array}$ & $\begin{array}{c}43.91 \pm \\
6.9\end{array}$ & $\begin{array}{c}52.82 \pm \\
3.92^{*}\end{array}$ & $\begin{array}{l}66.91 \pm \\
7.86^{* * *}\end{array}$ & $\begin{array}{l}85.93 \pm \\
8.20^{* * *}\end{array}$ \\
\hline
\end{tabular}

Notes: All pharmacokinetic parameters were calculated by noncompartment analysis using PK package in $R$, with variability calculated using bootstrap $t$ method.

AUCs are compared by one way ANOVA with Newman-Keuls multiple comparison test to compare all the groups. The results in the table show the comparison between each transporter genotype vs. WT. ${ }^{*} \mathrm{p}<0.05,{ }^{* * *} \mathrm{p}<0.001$.

$\mathrm{C}_{\max }$ of the $\mathrm{dKO}$ and WT is compared using Mann Whitney Test, $* \mathrm{p}<0.0 .5$. 


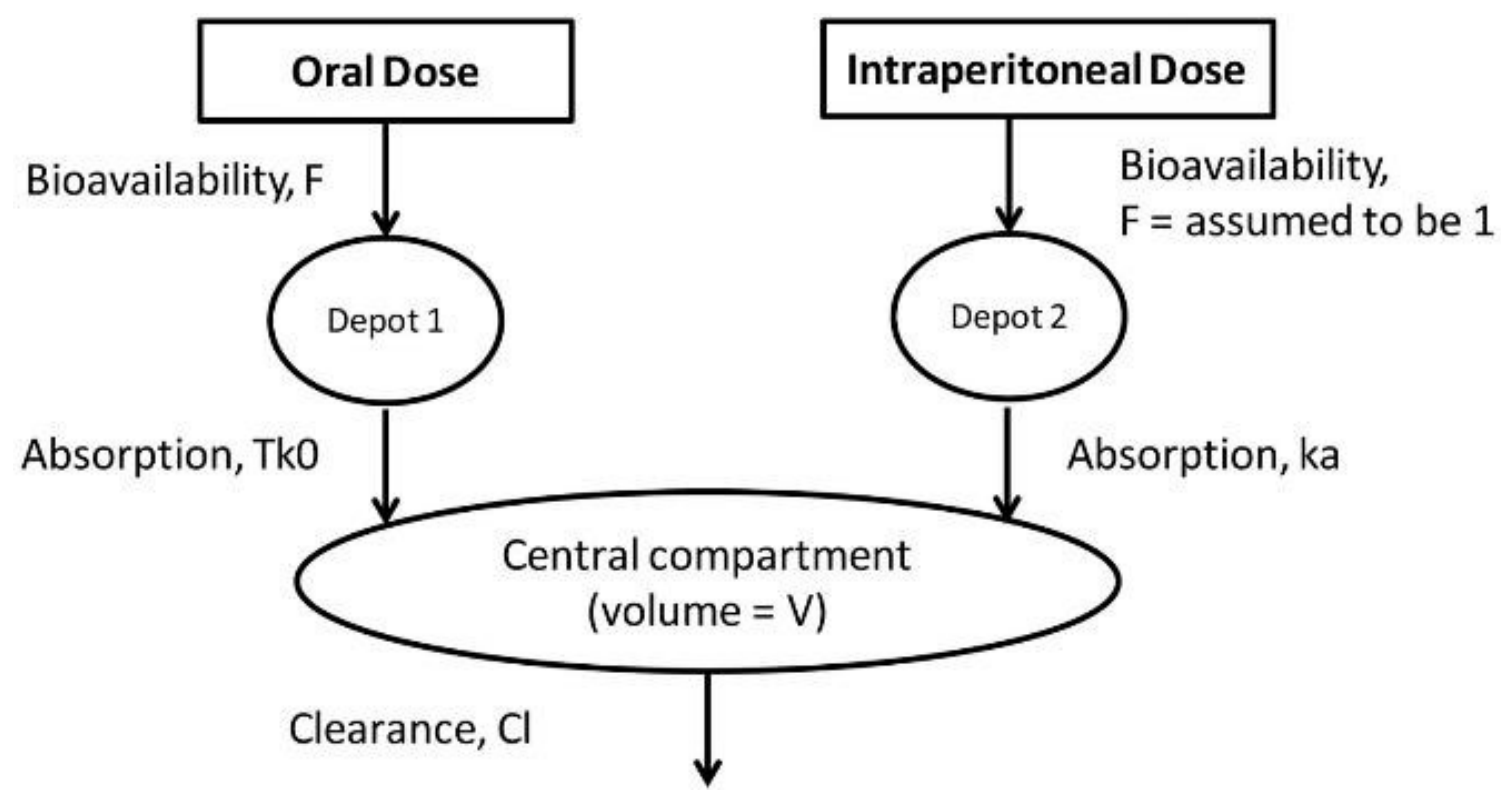

Figure 3-3. Schematic diagram of the final population model

Note: Ketamine one-compartmental distribution with zero order absorption for PO and first-order absorption for the IP dosing and first-order elimination from the central compartment 

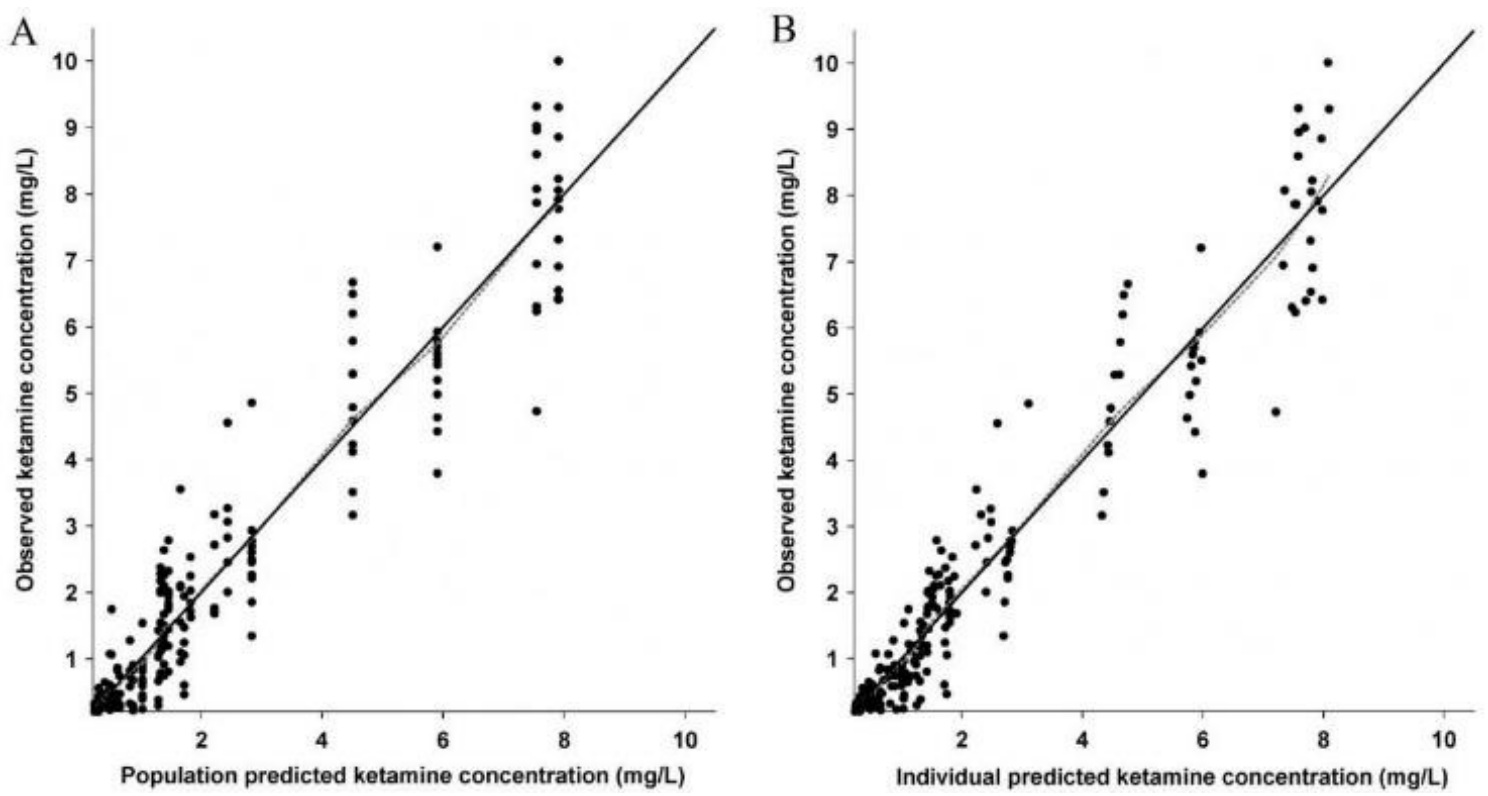

Figure 3-4. Diagnostic plots - observed vs. population and individual predicted ketamine concentrations

Notes: Observed ketamine concentration versus population predicted (A) and (B) individual predicted ketamine concentration for the final ketamine population PK model. The solid line represents the line of identity and the gray line represent the spline of the model. The plots show that the spline is very close to the line of identity, which implies that the final model was able to successfully predict the observed ketamine concentrations in serum. 

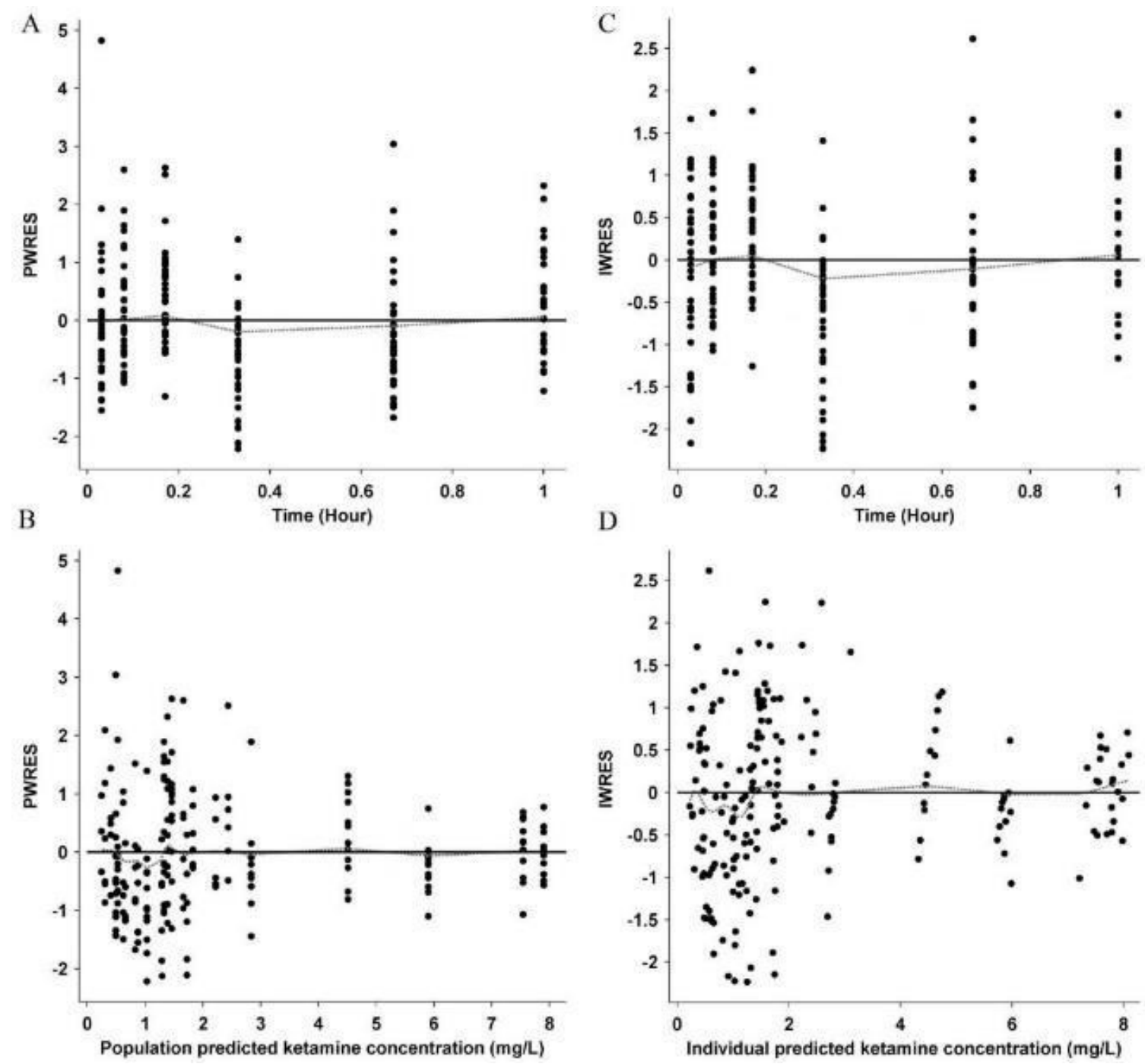

Figure 3-5. Diagnostic residual plots

Notes: (A) \& (B) - population weighted residual (PWRES) vs. time and predicted ketamine concentration, respectively. (C) \& (D) - Individual weighted residual (IWRES) vs. time and predicted concentration, respectively. The solid line represents the reference line at zero and the gray line represents the spline of the model. The plots show there was no systematic bias in the model over time or concentration range. Majority of the population residuals were equally distributed around zero and within \pm 2 , which indicates that the predicted data is within an acceptable range to the observed data. 

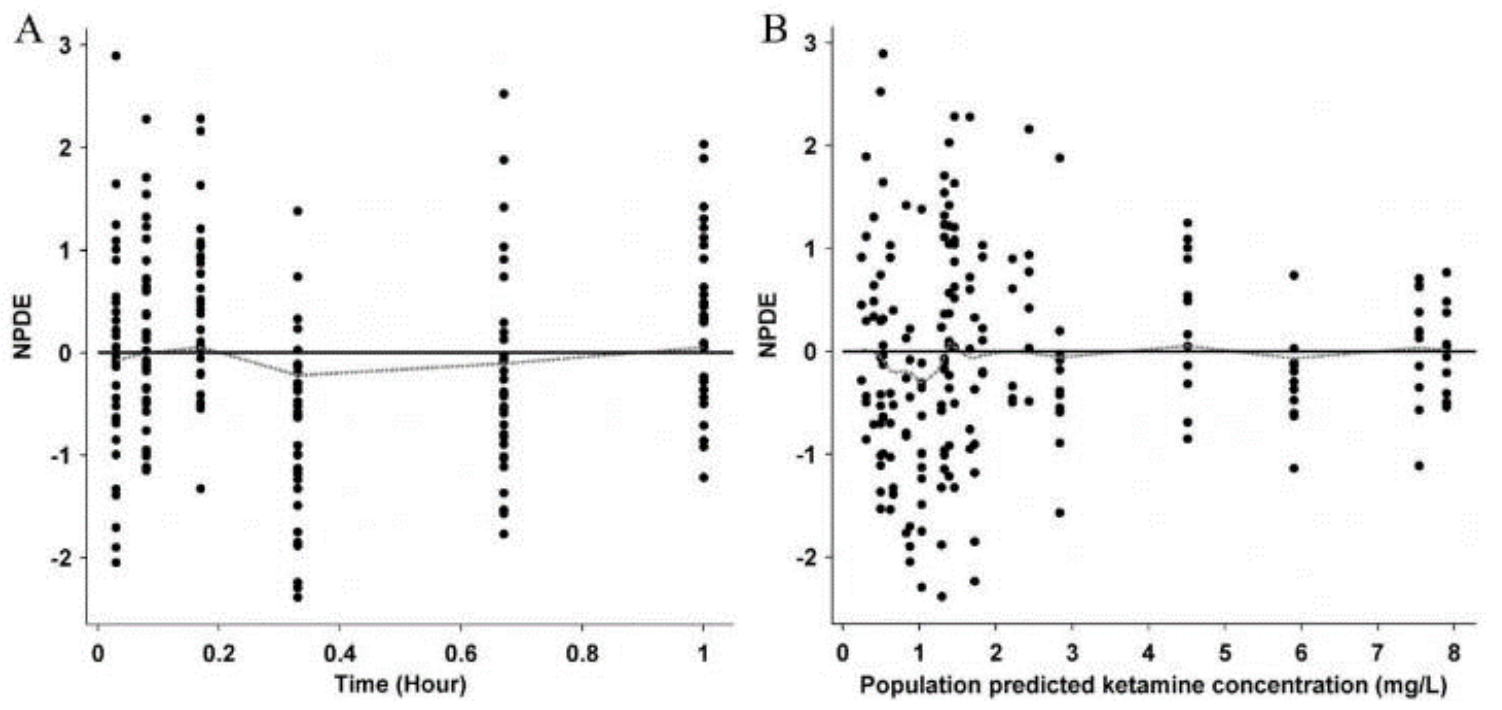

Figure 3-6. Normalized prediction distribution error (NPDE) plots

Notes: (A) NPDE vs. time and predicted ketamine concentration, respectively. (B) NPDE vs. time and predicted concentration, respectively. The solid line represents the reference line at zero and the gray line represents the spline of the model. The plots show a lack of model misspecification for the final model. 
Table 3-2. Population PK parameter estimates

\begin{tabular}{|c|c|c|c|}
\hline $\begin{array}{l}\text { Parameters } \\
\text { (Units) }\end{array}$ & $\begin{array}{c}\text { Mean Parameter } \\
\text { Estimates }\end{array}$ & S.E. & p-Value \\
\hline F_pop & 0.175 & 0.0072 & \\
\hline$\theta$ Pgp KO & 0.227 & 0.062 & 0.00028 \\
\hline$\theta \mathrm{dKO}$ & 0.514 & 0.062 & $<1 \mathrm{e}-10$ \\
\hline Tरे0_pop (1/hr) & 0.104 & 0.0092 & \\
\hline F2 pop & 1 Fixed & N.E. & \\
\hline ka_pop $(1 / \mathrm{hr})$ & 19.1 & 1.6 & \\
\hline V1_pop (L/Kg) & 9.32 & 0.35 & \\
\hline CL_pop $(\mathrm{L} / \mathrm{hr} / \mathrm{Kg})$ & 20.2 & 0.72 & \\
\hline \multicolumn{4}{|l|}{$\omega_{-} \mathrm{BSV}$} \\
\hline $\mathrm{F}^{-}$ & 0.0276 & 0.077 & \\
\hline Tk0 & 0.32 & 0.077 & \\
\hline F2 & N.E. & N.E. & \\
\hline $\mathrm{ka}$ & 0.091 & 0.14 & \\
\hline V1 & 0.0857 & 0.061 & \\
\hline CL & 0.0781 & 0.056 & \\
\hline \multicolumn{4}{|l|}{$\sigma-\mathrm{RUV}$} \\
\hline Proportional & 0.341 & 0.022 & \\
\hline $\begin{array}{l}\mathrm{F}_{-}(\mathrm{WT} \text { and Bcrp } \\
\mathrm{KO})\end{array}$ & 0.175 & 0.0072 & \\
\hline$F_{-}(\operatorname{Pgp} \mathrm{KO})$ & 0.22 & 0.011 & \\
\hline $\mathrm{F}^{-}(\mathrm{dKO})$ & 0.293 & 0.015 & \\
\hline
\end{tabular}

Notes: F - oral bioavailability; F_pop - Population mean oral bioavailability; $\theta$ _Pgp KO and $\theta \_\mathrm{dKO}$ - estimated coefficient of the effect of categorical covariate Pgp KO and $\mathrm{dKO}$ on the oral bioavailability; Tk0_pop - population mean oral zero order absorption rate constant; F2_pop - population mean intraperitoneal bioavailability which was assumed to be 1; ka_pop - population mean first order absorption rate constant for i.p route; V1_pop - population mean volume of distribution; CL_pop - population mean clearance; $\omega$ BSV - between subject variability; $\sigma$ - RUV - residual unexplained variability; N.E. - not estimated 
compartment analysis was not observed with the population approach likely due to variability in the result. However, both Pgp KO and dKO genotypes had a significant impact on population bioavailability $(\mathrm{p}<0.001)$. Oral bioavailability $(\mathrm{F})$ for the WT population was $17.5 \%$, which significantly increases to $22 \%$ in the Pgp KO mice, and increases further to $29 \%$ in the dKO mice. Hence, altered ketamine PK were observed following oral administration to mice lacking Pgp singly or in combination with Bcrp. This difference translates to a difference in the post-hoc estimated AUC between these groups.

\section{Ketamine-induced duration of loss of righting reflex (dLORR) was significantly increased in mice lacking Bcrp and Pgp}

The difference in PO PK between WT and Bcrp and/or Pgp knockout mice, and higher plasma and CSF abundance of ketamine in the $\mathrm{dKO}$ compared to WT rats led us to investigate if ketamine pharmacodynamics are also affected by Bcrp and Pgp. We used the ketamine-induced loss of righting reflex (LORR) model in the mouse to study the effect of Bcrp and Pgp on ketamine pharmacodynamics. Following itraperitoneal ketamine, the median dLORR in the dKO mice was longer than in WT (2.6-fold), Bcrp KO (2.1-fold), and Pgp KO (1.3-fold) mice (Figure 3-7A and Table 3-3). In addition, the median dLORR was 2-fold longer in the Pgp KO compared to the WT mice. These results indicate that both Pgp and Bcrp influence ketamine pharmacodynamics as measured by ketamine-induced dLORR.

To determine whether ketamine brain concentrations were different in the transporter KO vs. WT mice we first administered IP ketamine and measured brain and serum concentrations at 15 minutes after dosing ( $n=3$ in each genotype group). Our HPLC-UV analysis shows a median brain/serum ratio of 1.32 in all genotypes and there is no significant difference in brain/serum ketamine concentration between genotypes ( Figure 3-8). To understand if the total concentration of ketamine and its metabolites are higher in the knockout mice compared to WT mice, we performed a whole brain homogenate ${ }^{3} \mathrm{H}$-ketamine radioactivity study. We administered IP the standard anesthetic dose of ketamine $(100 \mathrm{mg} / \mathrm{kg})$ spiked with ${ }^{3} \mathrm{H}$-ketamine and measured the ${ }^{3} \mathrm{H}$-ketamine brain/serum ratio at $1.25 \mathrm{hr}$. Brain/serum ${ }^{3} \mathrm{H}$-ketamine radioactivity ratio was not significantly different between genotypes with a median ratio of 1.45 in all mice. This result is similar to what we observed with the HPLC-UV analysis. Although there was a measurable increase in the level of ${ }^{3} \mathrm{H}$-ketamine in the brains of Pgp and Pgp/Bcrp dKO mice, the difference between genotypes did not reach statistical significance ( Figure 3-8), despite the remarkable impact of the transporters on ketamine's pharmacodynamic effect (Figure 3-7A), and the significant increase in CSF ketamine (measured by LC-MS/MS) in Pgp/Bcrp dKO rats (Figure 3-1). Although Pgp KO vs $\mathrm{dKO}$ brain ${ }^{3} \mathrm{H}$ - ketamine radioactivity difference reached statistical significance, the median $\mathrm{dKO} / \mathrm{Pgp} \mathrm{KO}$ radioactivity ratio is 0.85 , which could be due to differences in metabolite concentration between these two genotypes or even variability associated with analysis, dosing or other reasons. 
A

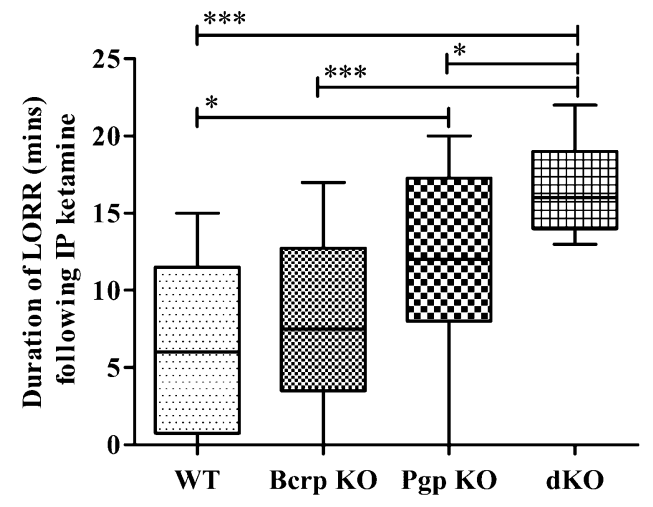

B

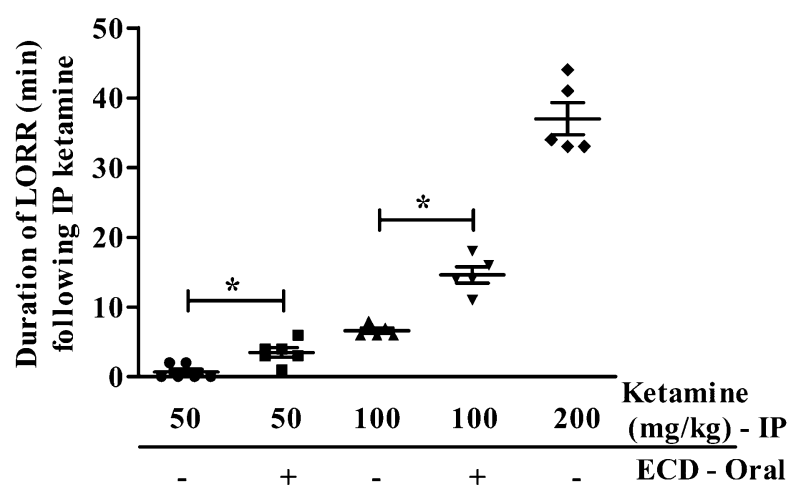

Figure 3-7. The duration of IP ketamine induced loss of righting reflex (dLORR) significantly increases in mice with absent or inhibited Pgp and Bcrp

Notes: (A) Comparison of the duration of ketamine (100 $\mathrm{mg} / \mathrm{kg}$ body weight, IP) induced dLORR in WT, Bcrp KO, Pgp KO and dKO FVB mice. (B) The duration of ketamine $(50,100$ and $200 \mathrm{mg} / \mathrm{kg}$ body weight, intraperitoneal) induced LORR in WT mice with or without $1.5 \mathrm{hr}$ pretreatment with elacridar (ECD) $(100 \mathrm{mg} / \mathrm{kg}$ body weight). Results are expressed as median dLORR \pm range. Significance is calculated using Mann-Whitney test. $* \mathrm{p}<0.05, * * \mathrm{p}<0.01, * * * \mathrm{p}<0.001$ 
Table 3-3. Descriptive statistics of ketamine induced dLORR in male FVB mice (WT, Bcrp ${ }^{-/}$, Pgp $^{-/-}$, Bcrp-Pgp ${ }^{-/-}$)

\begin{tabular}{lcccc}
\hline $\begin{array}{l}\text { dLORR Time } \\
\text { (minutes) }\end{array}$ & $\begin{array}{c}\text { WT } \\
(\mathbf{n = 1 2})\end{array}$ & $\begin{array}{c}\text { Bcrp KO } \\
(\mathbf{n}=\mathbf{1 2})\end{array}$ & $\begin{array}{c}\text { Pgp KO } \\
(\mathbf{n = 1 2})\end{array}$ & $\begin{array}{c}\mathbf{d K O} \\
(\mathbf{n = 1 1})\end{array}$ \\
\hline Minimum & 0.0 & 0.0 & 0.0 & 13.0 \\
25\% Percentile & 0.75 & 3.5 & 8.0 & 14.0 \\
Median & 6.0 & 7.5 & $\mathbf{1 2 . 0 *}$ & $\mathbf{1 6 . 0 * * *}$ \\
75\% Percentile & 11.5 & 12.75 & 17.25 & 19.0 \\
Maximum & 15.0 & 17.0 & 20.0 & 22.0 \\
Mean & 6.5 & 8.0 & 11.58 & $\mathbf{1 6 . 7 3}$ \\
Std. Deviation & 5.45 & 5.69 & 6.20 & 3.13 \\
Std. Error & 1.57 & 1.64 & 1.79 & 0.94 \\
Lower 95\% CI of mean & 3.04 & 4.38 & 7.64 & 14.62 \\
Upper 95\% CI of mean & 9.96 & 11.61 & 15.52 & 18.83 \\
\hline
\end{tabular}

Note: Significance is calculated using Mann-Whitney test by comparing the median dLORR in each genotype with the median dLORR in the WT mice. ${ }^{*} \mathrm{p}<0.05$, *** $\mathrm{p}<$ 0.001. CI $=$ Confidence interval 

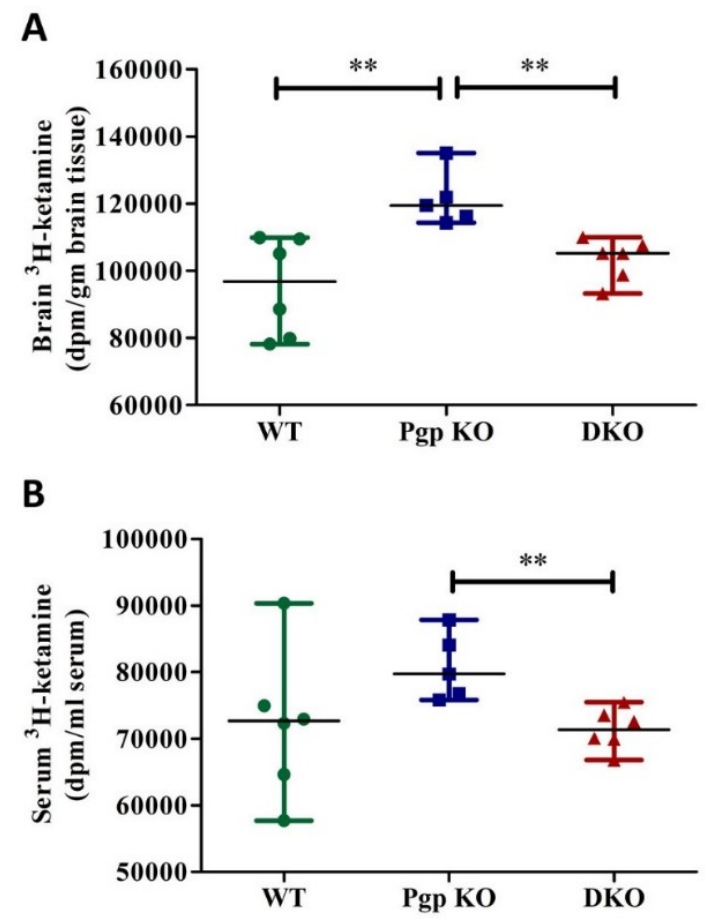

C

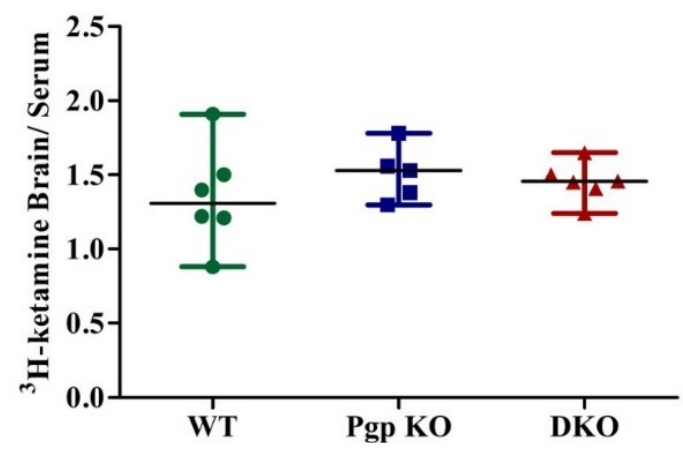

D

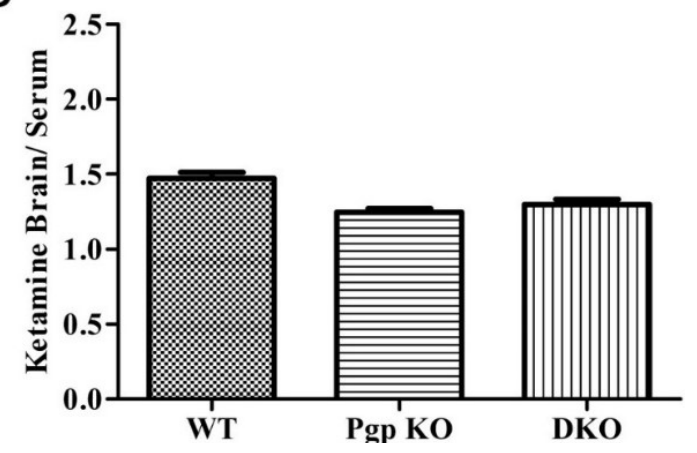

Figure 3-8. Ketamine brain/serum ratios in wild-type, PgpKO and Pgp/BcrpdKO mice

Note: Brain ketamine in mice after IP dosing with ketamine $(100 \mathrm{mg} / \mathrm{kg})+1 \mu \mathrm{Ci}$ ketamine tracer. Results are expressed as median radioactivity $(\mathbf{A}, \mathbf{B})$ or brain/serum radioactivity ratio \pm range $(\mathbf{C})$. Brain/serum concentration ratio $(\mathbf{D})$ was measured by HPLC-UV based method after single intraperitoneal dose of ketamine $(100 \mathrm{mg} / \mathrm{kg})$.

Significance is calculated using Mann-Whitney test. ${ }^{*} \mathrm{p}<0.05, * * \mathrm{p}<0.01,{ }^{* * *} \mathrm{p}<0.001$ 


\section{Pharmacological inhibition of Bcrp and Pgp by elacridar increased IP ketamine- induced dLORR in WT mice}

Since we observed altered PK and PD of ketamine in mice with genetic deletion of Bcrp and/or Pgp, we investigated whether ketamine PD were also altered by pharmacological inhibition of these efflux transporters by using elacridar as a dual inhibitor of Bcrp and Pgp. Elacridar dosing and time schedules for pretreatment were based on previous reports that clearly demonstrated loss of Bcrp and Pgp function at the dose selected [269-271]. Wild-type mice were pretreated with vehicle or $100 \mathrm{mg} / \mathrm{kg}$ elacridar and $1.5 \mathrm{hrs}$ later ketamine was dosed IP at 50,100 and $200 \mathrm{mg} / \mathrm{kg}$ ( $\mathrm{n}=6$ per dose group) and duration of LORR measured. The results in WT mice of ketamine treatment alone (Figure 3-7B, Table 3-4) demonstrated a dose-proportional increase in druginduced dLORR from 50 to $100 \mathrm{mg} / \mathrm{kg}$, but a disproportionately longer increase in dLORR at $200 \mathrm{mg} / \mathrm{kg}$. Pretreatment with elacridar significantly increased dLORR in the WT mice treated with either 50 or $100 \mathrm{mg} / \mathrm{kg}$ ketamine. Based on the exceedingly long dLORR with $200 \mathrm{mg} / \mathrm{kg}$ ketamine alone, co-treatment at this dose with elacridar was not attempted to avoid toxicity. These results show that inhibition of Bcrp and Pgp with the dual inhibitor elacridar significantly increased ketamine-mediated dLORR in WT mice.

\section{Ketamine-induced dLORR was significantly longer in dKO vs. WT mice after oral dosing of $200 \mathrm{mg} / \mathrm{kg}$ ketamine}

Because ketamine can be taken orally, we compared the effect of the drug transporters on its pharmacodynamic effect following oral administration. Oral ketamine $(100 \mathrm{mg} / \mathrm{kg}$ ) dosing was insufficient to cause a LORR in any of the mice (data not shown) likely because the ketamine serum concentration was lower following oral vs. IP dosing (four-fold lower in dKO mice and seven-fold lower in WT mice) (Table 3-1). Following oral ketamine $(200 \mathrm{mg} / \mathrm{kg})$ treatment, WT mice were all awake within 4 minutes, while one of the $\mathrm{dKO}$ mice died and the others needed to be warmed to wake them after 30 minutes, preventing further analysis of this dose (not shown).

\section{Ketamine accumulation is lower in MDCKII cells transfected with human BCRP and Pgp compared to WT MDCKII cells}

The time course of 3H-ketamine cellular uptake was first compared between MDCKII cells and MDCKII-PGP-BCRP cells stably expressing the human transporters [262]. The 3H-ketamine cellular concentration was significantly greater in MDCKII parental cells compared to cells expressing BCRP and PGP at all time points, but uptake reached equilibrium by 20 minutes (Figure 3-9A). Likewise, the 3H-ketamine intracellular concentration measured at 20 minutes was higher in the parental MDCKII cells compared to the dual BCRP/Pgp expressing cells between 0.1 to $5 \mu \mathrm{M}$ concentrations but not at $10 \mu \mathrm{M}$ concentration (Figure 3-9B). The data also indicated that the magnitude of difference in intracellular $3 \mathrm{H}$-ketamine was greater at lower concentrations vs. higher concentrations between the MDCKII cells with and without 
Table 3-4. Descriptive statistics of ketamine induced loss of righting reflex (LORR) in male WT FVB mice with or without $100 \mathrm{mg} / \mathrm{kg}$ oral elacridar 1.5 hours before intraperitoneal ketamine dosing

\begin{tabular}{|c|c|c|c|c|c|}
\hline $\begin{array}{l}\text { dLORR Time } \\
\text { (minutes) }\end{array}$ & $\begin{array}{c}50 \\
\mathrm{mg} / \mathrm{kg} \\
(\mathrm{n}=6)\end{array}$ & $\begin{array}{c}50 \mathrm{mg} / \mathrm{kg} \\
+\mathrm{ECD} \\
(\mathrm{n}=6)\end{array}$ & $\begin{array}{c}100 \\
\mathrm{mg} / \mathrm{kg} \\
(\mathrm{n}=5)\end{array}$ & $\begin{array}{c}100 \mathrm{mg} / \mathrm{kg} \\
+\mathrm{ECD} \\
(\mathrm{n}=5)\end{array}$ & $\begin{array}{c}200 \\
\mathrm{mg} / \mathrm{kg} \\
(\mathrm{n}=5)\end{array}$ \\
\hline Minimum & 0.00 & 1.00 & 6.00 & 11.00 & 33.00 \\
\hline 25\% Percentile & 0.00 & 2.50 & 6.00 & 12.50 & 33.00 \\
\hline Median & 0.00 & $3.50 *$ & 6.00 & $14.00 *$ & 34.00 \\
\hline $75 \%$ Percentile & 2.00 & 4.50 & 7.50 & 17.00 & 42.50 \\
\hline Maximum & 2.00 & 6.00 & 8.00 & 18.00 & 44.00 \\
\hline Mean & 0.67 & 3.50 & 6.60 & 14.60 & 37.00 \\
\hline Std. Deviation & 1.03 & 1.64 & 0.89 & 2.61 & 5.15 \\
\hline Std. Error & 0.42 & 0.67 & 0.40 & 1.17 & 2.30 \\
\hline Lower $95 \%$ CI of mean & -0.42 & 1.78 & 5.49 & 11.36 & 30.61 \\
\hline Upper $95 \%$ CI of mean & 1.75 & 5.22 & 7.71 & 17.84 & 43.39 \\
\hline
\end{tabular}

Note: Significance is calculated using the Mann-Whitney test by comparing each ECD treated ketamine dose group by their corresponding no ECD pretreatment group. ${ }^{*} \mathrm{p}<$ 0.05 . 

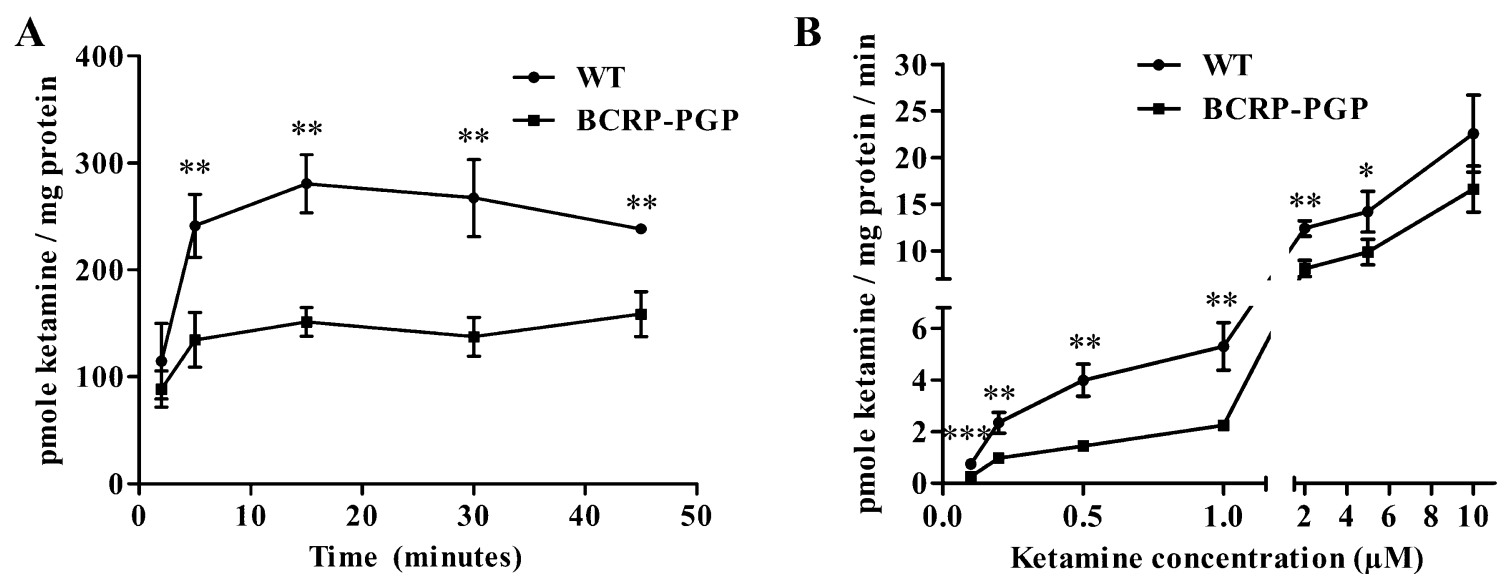

Figure 3-9. $\quad{ }^{3} \mathrm{H}$-ketamine uptake is lower in the MDCKII BCRP-PGP cells compared to MDCKII WT cells

Notes: (A) Time dependent uptake of 3H-ketamine in WT and BCRP-PGP transfected MDCKII cells. Ketamine's intracellular concentration at each time point was compared between the WT and BCRP-PGP cells using an unpaired t-test, $* * \mathrm{p}<0.01$. (B) $3 \mathrm{H}$ ketamine uptake in WT and BCRP-PGP transfected MDCKII cells at different ketamine concentrations. The intracellular concentration of $3 \mathrm{H}$-ketamine at each treatment was compared between the WT and BCRP-PGP cells using an unpaired t-test, ${ }^{*} \mathrm{p}<0.05, * *$ $\mathrm{p}<0.01$ and ${ }^{* * *} \mathrm{p}<0.001$. 
drug transporters. In support of these findings, Keiser et al (2018) recently published (while this manuscript was under review) in vitro studies showing that ketamine is a Pgp substrate [98].

\section{Discussion}

Following the unexpected finding that the concentration of the anesthetic ketamine was elevated in the CSF and plasma of Pgp-Bcrp dKO compared to wild-type rats, this study was performed in mice to determine whether PK and PD of ketamine is affected by these transporters, and thus, if ketamine is a possible dual substrate of these transporters. We observed ketamine oral exposre increased in the following order: WT $<$ Bcrp KO $<$ Pgp KO $<$ dKO (Figure 3-2). Likewise, ketamine-induced dLORR was significantly longer in the $\mathrm{dKO}>\mathrm{Pgp} \mathrm{KO}>\mathrm{Bcrp} \mathrm{KO}>\mathrm{WT}$ mice (Figure 3-7). In summary, absence of Pgp/Bcrp decreased IP ketamine-induced dLORR (Figure 3-7), increased ketamine oral clearance (Figure 3-2D, Table 3-2), and inhibition of these transporters by elacridar increased IP ketamine induced-dLORR in wild-type mice (Figure 3-7B), demonstrating ketamine is an apparent substrate for both transporters, and also indicating a possible drug-drug interaction of ketamine with clinical Bcrp-Pgp inhibitors, such as elacridar.

There is mixed evidence in the literature that ketamine might interact with Pgp or Bcrp. Ketamine was previously screened for inhibitory activity against BCRP and PGP [272] using MDCKII cells stably transfected with human BCRP or MDR1 (PGP) and found to not inhibit either transporter. However, high concentrations of cyclosporine increased the dLORR in mice [261], which could be due cyclosporine's ability to inhibit ketamine metabolizing Cyps as well as Pgp and Bcrp. The finding that female vs. male rats show a longer ketamine induced dLORR [273] might also reflect the fact that there is significant sexual dimorphism in rodent Bcrp expression with males vs. females having significantly higher levels of Bcrp in liver, intestine and kidney [215]. Co-administration of morphine (a Pgp substrate) and ketamine increased ketamine's brain concentration [260], which would be consistent with the ketamine competing with morphine for Pgp transport.

We used dLORR to compare the ketamine-induced anesthetic/hypnotic effect in mice with different transporter genotypes as dLORR can be used to assign dosedependent pharmacodynamic effects [267]. Although we observed a significantly increased IP ketamine induced dLORR in the dKO mice compared to WT, Bcrp KO and Pgp KO mice (Figure 3-7A), we did not find a significant increase in serum PK after IP ketamine dosing (Figure 3-2.A) in the transporter single and dKO mice. However, it is not unprecedented for some Pgp substrates to not show systemic PK differences between WT and Pgp KO mice following IP dosing (e.g., dasatinib) [268]. Although ketamine levels were significantly higher in the plasma of Pgp/Bcrp dKO rats $\sim 7$ min after IP dosing (Figure 3-1), we were unable to detect a significant difference in ketamine PK following IP dosing of WT or single transporter KO mice (Figure 3-2A). This might have resulted from several factors including inter-species variability in 
metabolism/transport and the difference in blood collection time after ketamine between the two studies. PK parameters of ketamine in mice are not extensively reported in the literature. From the concentration-time data reported [267] after a $200 \mathrm{mg} / \mathrm{kg}$ IP dose, and [274] after a $10 \mathrm{mg} / \mathrm{kg}$ IP dose in mice, ketamine's plasma half-life (t1/2) is approximately 25 minutes, which is similar to the results obtained in our study (Table 3-2). The $\mathrm{C}_{\max }$ after IP dosing of $10 \mathrm{mg} / \mathrm{kg}$ ketamine was reported between $0.55 \mu \mathrm{g} / \mathrm{ml}$ and $1 \mu \mathrm{g} / \mathrm{ml}[274,275]$, while we observed a $C_{\max }$ of $7.88 \pm 1.38 \mu \mathrm{g} / \mathrm{ml}$ (Table 3-1) after $100 \mathrm{mg} / \mathrm{kg}$ IP dose of ketamine, which is within the dose linear range of the reported data. Whereas, Sato et al., after $200 \mathrm{mg} / \mathrm{kg}$ IP ketamine dosing in C57Bl/6 mice, reported a highest concentration of approximately $30 \mu \mathrm{g} / \mathrm{mL}$ at 10 minutes [267], indicating a possible more than dose proportional increase in ketamine exposure beyond $100 \mathrm{mg} / \mathrm{kg}$ dose. Although ketamine bioavailability in humans after oral dosing has been reported to be between 7 and 20\% [79, 255, 257], bioavailability after oral dosing in mice is not well established. Assuming 100\% bioavailability after IP dosing, bioavailability after oral dosing in WT mice falls into the same range (F_Pop, Table S1) in our study to that observed in humans.

We did not find a significant difference in ketamine brain/ plasma ratio between the mice with different transporter genotypes (Figure 3-8), and the increase of CSF concentrations in Pgp/Bcrp KO vs. WT rats can largely be explained by the increase in plasma concentrations (Figure 3-1). These data suggest blood brain barrier Pgp/Bcrp do not (or may not) impair ketamine brain concentration. So why are the effects of Pgp/Bcrp on ketamine's pharmacodynamics consistent following either IP or oral dosing, mirrored by their dual inhibitor elacridar, and consistent with oral ketamine PK, but inconsistent with ketamine's brain concentration? There are several alternative reasons for not finding a measurable effect of the transporters on ketamine brain concentration at 15 minutes (HPLC-UV) and $1.25 \mathrm{hr}$ (radioactivity). First, most studies that have shown a significant effect of Pgp/Bcrp on substrate brain concentrations in vivo have measured drug brain levels 4-24 hrs after drug administration to allow brain accumulation and hence maximize the difference in brain substrate concentration in the KO vs. WT mice. However, because of ketamine's rapid clearance rate (estimated t1/2=25 min) (Table 3-1), brain concentrations would not be measureable hours after dosing. Second, ketamine is a high permeability rapid clearance drug and the magnitude of effect of Pgp and Bcrp is typically greater for substrates with poor apparent permeability coefficients [31], so the magnitude of transporter effect on this type of substrate would be assumed to be small. Indeed, terfenadine, another drug with high passive permeability that is a Pgp substrate, showed no difference in brain uptake clearance, measured by in situ brain perfusion, between WT and Pgp-KO mice [97]. Third, it was previously shown that i.v administered ketamine shows significant regional differences in brain exposure (that are thought to be pertinent to its effect on anesthesia) during loss of righting reflex in rats, and the difference minimizes as the rats regained righting reflex [276], which might explain its PD effect, but which would not be captured by measuring ketamine in total brain homogenate (as we did in this study). Hence, it is possible that regional concentration difference of ketamine could be driving its dLORR effect, and ketamine plasma and total brain concentration may not be a good predictor of its PK/PD relationship in the dLORR model. Fourth, we administered ketamine as the racemic 
mixture but it is entirely possible that the S- and R-ketamine enantiomers (that have enantiomer specific pharmacology) might interact differently with the transporters (beyond the scope of this paper). Finally, we think the striking effect of transporter genotype on ketamine PD, but not systemic or total brain distribution, for brain acting drugs like ketamine that have high passive permeability may, in fact point to the potential for missing a Pgp or Bcrp effect if only the PK is measured and used to predict whether these transporters would influence the drug's CNS pharmacodynamic activity.

A population PK model was used for simultaneous analysis of IP and PO PK data to enable estimation of fixed parameters and sources of variability. Assuming a $100 \%$ bioavailability after IP dosing (F2 = 1, Table 3-2), population PO bioavailability in the current study was estimated to be $17.5 \%$, which is in accordance to the published reports of ketamine bioavailability in humans after oral dosing. Population analysis of IP and PO data together identified a change in oral bioavailability in the Pgp and dKO groups. The mean population clearance for ketamine was found to be $20.2 \mathrm{~L} / \mathrm{hr} / \mathrm{kg}$ and volume of distribution $9.32 \mathrm{~L} / \mathrm{kg}$, suggesting ketamine has very high clearance and volume of distribution, which is in accordance with previously published results [253].

While our in vivo data clearly shows Pgp and Bcrp alter the POPK and dLORR of ketamine, we wanted to know if the same is observed in vitro. Previously published results show ketamine does not have any interaction with PGP, as substrate or inhibitor. However, our data clearly indicates, in a dual BCRP and PGP transfected MDCKII cell line, these transporters can effectively reduce ketamine uptake in MDCKII cells at low ketamine concentration. We think both the selection of ketamine concentration and also time of in vitro assay will have significant effect on the outcome. Also, our in vitro data suggests that effect of these transporters will be significant at ketamine exposure lower than $10 \mu \mathrm{M}$ or $2.4 \mu \mathrm{g} / \mathrm{ml}$. A higher clinical ketamine concentration is required for anesthetic effect of ketamine, whereas, lower concentrations are useful for antidepressant effect and also often achieved in the ketamine abusers. Thus, our data warrants a possible drug-drug or food-drug interaction for antidepressant use of ketamine and in ketamine abusers.

The finding that ketamine appears to be a dual Pgp/Bcrp substrate has a number of important clinical implications. Ketamine is an anesthetic widely used in animal studies as well as human pediatric settings where a short-acting rapid-onset anesthesia is needed. Importantly, it was recently recognized that ketamine can elicit rapid-onset longacting anti-depressant activity. However, ketamine is also a significant drug of abuse, particularly in Asian countries. Notably, reports have also appeared in the past few years that chronic ketamine abusers develop serious lower urinary tract symptoms, voiding problems, ulcerative cystitis, and significant damage to the urinary tract [252], although there may be individual differences in vulnerability. Both Pgp and Bcrp are expressed in human kidney at the proximal tubule epithelium where they move substrates into the urine, and both transporters may be expressed in bladder urothelium [277] at the apical epithelium. Given our findings that ketamine is an apparent Pgp/Bcrp substrate it is tempting to speculate that BCRP genetic variation, particularly the BCRP Q141K allele that leads to loss of functional BCRP protein and that has a high frequency in some 
populations [111] may influence susceptibility to ketamine-induced urinary tract damage. Equally important is the potential for co-administered Pgp/Bcrp substrates or inhibitors to affect both ketamine bioavailability and urinary tract damage. Indeed, our studies with the dual Pgp/Bcrp inhibitor elacridar demonstrated that pharmacological inhibition of the transporters can affect ketamine PK. Further, cyclosporine, a known Pgp/Bcrp as well as Cyp inhibitor has similarly been shown to affect ketamine dLORR in mice [261]. Many therapeutically used drugs and chemicals present in food are inhibitors of Bcrp and Pgp [259]. Thus, our results suggest a potential interaction of ketamine with these Bcrp-Pgp inhibitors. Moreover, the proposed use of ketamine as an antidepressant may require dosing with other antidepressant drugs, some of which are known inhibitors of these transporters [278].

We conclude that Bcrp and Pgp can affect the PK and PD of ketamine in mice. Using a pharmacological inhibitor of Bcrp and Pgp function our data clearly demonstrate DDI potential of ketamine with inhibitors of Bcrp and Pgp. Further studies should be carried out to understand the impact of these transporters on ketamine PK, PD and DDI potential in humans and whether persons with polymorphic Bcrp taking Pgp inhibitors have an increased risk of ketamine-mediated toxicity. 


\section{CHAPTER 4. PHEOPHORBIDE A: IN VIVO BCRP SUBSTRATE TO IDENTIFY ORAL DRUG-DRUG INTERACTIONS}

\section{Introduction}

The xenobiotic efflux transporter, breast cancer resistance protein (BCRP), encoded by the ABCG2 gene, is an ATP-binding cassette (ABC) sub-family G member 2 half transporter that is well characterized for its effect on pharmacokinetics and pharmacodynamics of multiple drugs [6]. BCRP was originally discovered and named by Doyle et al. in breast cancer cell line (MCF7) conferring resistance to anthracycline anticancer drugs [62]. Though originally named for its expression on breast cancer cells, BCRP is expressed almost ubiquitously on the apical membrane of endothelial and/or epithelial cells, where it limits tissue or organ permeability of its substrates [103, 104], as well as on stem cells, where BCRP can provide some survival advantages [109, 129]. Expression of BCRP is now well-established in liver, kidney, intestine and blood-brain and blood-CSF barriers [135, 279]. It has a significant effect on the bioavailability of its substrates, administered orally, [177] as well as their brain permeability in mice lacking Bcrp or in presence of Bcrp inhibitors [94]. Bcrp is also known to efflux its substrates into milk at the mammary gland in rodents [280] and confer protection to the fetus at placenta [281]. A wide variety of drugs such as antivirals, antibiotics, calcium channel blockers and statins, as well as endogenous and dietary metabolites such as riboflavin, folic acid, uric acid, protoporphyrin IX, pheophorbide A and estrone-3-sulfate are substrates of Bcrp [6, 9, 54, 101, 124, 129]. Moreover, multiple clinically used drugs, such as tyrosine kinase inhibitor lapatinib, HIV protease inhibitor ritonavir, calcium channel blockers, immunosuppressant etc., are known inhibitors of this transporter [121].

Due to its wide range of substrate and inhibitor recognition, as well as significant effect on the systemic distribution of its substrates, Bcrp is recognized by the FDA and International Transporter Consortium (ITC) as an important transporter which is prone to potential clinical drug-drug interactions (DDI) [1, 25, 31, 80, 208]. Except for biopharmaceutical classification system class I (BCS class I, high solubility and high permeability) compounds, BCRP substrates (victim) are prone to BCRP inhibitor (perpetrator)-mediated DDI when they are dosed concomitantly, through the oral route $[1,71]$. It has also been recognized by FDA that the DDI liability related to BCRP needs to be especially considered if the victim drug has potential toxicity concerns in distributing tissues $[1,80]$. Therefore, a better understanding of systemic inhibition of BCRP is also required for substrates whose tissue distribution (such as in brain, kidney, etc.) is controlled by the BCRP transporter.

While ITC and FDA have detailed the decision tree and guidelines for determination of BCRP substrates, inhibitors and the possibility of clinical DDI [1, 25], it has also been recognized that clinically used probe-substrates such as rosuvastatin and sulfasalazine are not specific to BCRP [282-284]. Hence, multiple factors, such as enzyme inhibition or inhibition of other transporters such as OATP1B1, can lead to variability in the clinical observation of altered rosuvastatin / sulfasalazine disposition, 
thereby requiring careful consideration for better understanding of the results [71]. Moreover, due to lack of specificity of the clinical inhibitors of BCRP [71], as well as expression of multiple transporters in the cell lines used for identification of BCRP substrates and inhibitors $[279,285,286]$, translation of in vitro observations to in vivo is often challenging. In vivo assessment of Bcrp substrates have utilized transporter knockout models or imaging techniques such as positron emission tomography (PET) and gamma-scintigraphy which are known to have their own caveats [25]. Therefore, a suitable assay for Bcrp activity, utilizing a specific and, if possible, same probe, both in vitro and in vivo, that is also more economical and with potential high-throughput capabilities, will be of immense value.

Pheophorbide $\mathrm{A}(\mathrm{PhA})$ is a breakdown product (catabolite) of chlorophyll and is a specific dietary substrate of Bcrp [92, 287, 288], with an efflux ratio of 3.15 in cell lines overexpressing Bcrp [289]. It is a low solubility, low permeability compound with no reported metabolic instabilities. Therefore, $\mathrm{PhA}$ might be a potentially suitable candidate as an in vitro as well as in vivo probe for investigating Bcrp function according to the FDA and ITC guidelines [25] [1]. PhA is also a colored compound with absorption maxima between $670 \mathrm{~nm}$ and $680 \mathrm{~nm}$, and fluorescence emission maxima between 675 and $720 \mathrm{~nm}$ [290], with typical fluorescence spectra for in vitro studies being $400 \mathrm{~nm}$ (excitation) and $670 \mathrm{~nm}$ (emission). However, a higher excitation (654 nm) and emission $(750 \mathrm{~nm})$ has also been reported for $\mathrm{PhA}$ [291], indicating that $\mathrm{PhA}$ fluorescence can be detected at the near infrared (NIR) region, thereby making it a suitable candidate for in vivo fluorescence measurement in whole animals [292].

We therefore hypothesized that $\mathrm{PhA}$ can be utilized as an in vivo probe for assessment of DDI associated with the inhibition of Bcrp at the gastro-intestinal tract (GIT) in mice. We utilized a live animal fluorescence imaging technique, using PhA, to screen for orally administrated BCRP inhibitors and predict intestinal BCRP-mediated DDIs. Our results demonstrate that in vivo fluorescence imaging utilizing $\mathrm{PhA}$ can be used for identification of BCRP inhibitors in order to assess BCRP-mediated clinical DDIs.

\section{Materials and Methods}

\section{Chemicals and reagents}

The following chemicals and reagents were purchased: Pheophorbide A (Frontier Scientific, Newark, DE, USA), lapatinib and curcumin (Cayman Chemicals, Ann Arbor, MI, USA), elacridar (Astatech Inc., Bristol, PA, USA), pantoprazole (Santa Cruz Biotech, Dallas, TX, USA), sorafenib and dasatinib (Chemietek, Indianapolis, IN, USA), chlorophyll A and protoporphyrin IX (Sigma-Aldrich, St. Louis, MO). 


\begin{abstract}
Animals
Wild type (WT) and Bcrp KO Friend Virus B (FVB) male mice ( $\mathrm{n}=4$ each group, 10 weeks old) were purchased from Taconic Biosciences, Inc. (Hudson, NY) for the knockout mice study. For all other Bcrp inhibitor studies, control mice of FVB background ( $\mathrm{n}=4$ for control and each inhibitor treatment group) were purchased from The Jackson Laboratory (Bar Harbor, ME, USA). All mice were fed either regular diet (Purina catalog\# 5013, Largo, FL) or alfalfa-free (AFF) diet (Purina, catalog\# AIN-93G, Largo, FL) for at least three days to reduce background auto-fluorescence, and they were provided water ad libitum. All experimental procedures were approved by the Institutional Animal Care and Use Committee of St. Jude Children's Research Hospital in accordance with the U.S. National Institutes of Health guidelines.
\end{abstract}

\title{
Cell lines
}

Madin-Darby Canine Kidney (MDCKII) cells overexpressing human full length wild type BCRP and the parenteral cell lines were kindly provided by Dr. Alfred Schinkel and were cultured as described [293].

\section{Determination of Pheophorbide A concentration in rodent food pellet}

Six food pellets each from regular diet and AFF diet were weighed and soaked with half parts of water. After 30 minutes, one gram of food pellet was mixed with $5 \mathrm{~mL}$ methanol and stirred overnight for $\mathrm{PhA}$ extraction. The following day, samples were centrifuged at $15000 \mathrm{~g}$ for 15 minutes and the supernatant collected. The supernatant was further centrifuged, filtered and used for measurement of PhA using a modified HPLCFluorescence Detector (HPLC-RF)-based method published previously [294]. $40 \mu \mathrm{L}$ of the final supernatant was injected onto the HPLC system (Shimadzu Prominence, Kyoto, Japan) consisting of a LC-20AB quaternary high pressure gradient pump, SIL-20ACHT auto sampler and RF-10 AXL fluorescence-detector. Chromatographic separation of PhA was achieved using Hypersil ODS C18 column (150X4 mm; $5 \mu \mathrm{m}$ particle size) fitted with Hypersil ODS (C18) Javelin Guard Column (10X4 mm; $5 \mu \mathrm{m}$ particle size) and a gradient elution method with a run time of 10 minutes. The mobile phase for elution was $80 \%$ methanol in $0.5 \mathrm{M}$ ammonium acetate (Phase A) and $100 \%$ methanol (Phase B) with a gradient of: 0 to 2 minutes 60 to $95 \%$ methanol, 2 to 8 minutes $95 \%$ methanol, 8 to 9 minutes 95 to $60 \%$ methanol, and the run stopped at 10 minutes. Retention time for $\mathrm{PhA}$ was 3.5 minutes. The PhA peak was identified and fluorescence was measured at 400 $(\mathrm{Ex})$ and $670(\mathrm{Em}) \mathrm{nm}$. Analyte area was used for calibration and measurement of unknown concentrations against a known PhA concentration prepared in mobile phase A. The calibration range was $0.02 \mu \mathrm{M}$ to $10 \mu \mathrm{M}$. 


\section{Treatments}

Mice were kept on AFF diet for at least three days to reduce the background autofluorescence. WT and Bcrp KO mice (10 weeks, $n=4$ /group) were administrated $\mathrm{PhA}(10 \mathrm{mg} / \mathrm{kg})$ by oral gavage, and imaging or blood collection was performed at appropriate time points up to $6 \mathrm{hrs}$ after dosing. For the inhibitor study, oral doses of the inhibitors were selected based on clinical relevance or previously demonstrated effects (Table 1) and formulated for oral dosing by suspending an appropriate amount of the compound in $0.5 \%$ hydroxypropyl-methyl- cellulose (HPMC) and 1\% Tween 80 (Sigma, St. Louis, MO). Bcrp inhibitors elacridar (ECD) (100 mg/kg), lapatinib (LPB) $(90$ $\mathrm{mg} / \mathrm{kg}$ ), dasatinib (DSB) (10 mg/kg), sorafenib (SFB) $(40 \mathrm{mg} / \mathrm{kg})$, curcumin (CCM) (300 $\mathrm{mg} / \mathrm{kg}$ ) and pantoprazole (PPZ) $(40 \mathrm{mg} / \mathrm{kg})$ were administrated orally, one hour prior to oral dosing of $\mathrm{PhA}$. Imaging and blood collection was performed at appropriate times up to 6 hours after PhA dosing. All animals were fasted up to 6 hours after PhA dosing.

\section{In vivo fluorescence imaging}

Mice were anesthetized by isoflurane anesthesia (2\%, inhalation) and placed ventrally in the chamber of Xenogen IVIS 200 (PerkinElmer, USA) imaging system. Fluorescence images were obtained under anesthesia with a fixed exposure time of one second and NIR filter setting of $675 \mathrm{~nm}$ excitation and $840 \mathrm{~nm}$ emission. Mice were repetitively imaged at $0,1,2,3,4$ and 6 hours after $\mathrm{PhA}$ administration. Images were scaled to a maximum intensity of $1 \times 108$ photons $/ \mathrm{s} / \mathrm{cm} 2 / \mathrm{sr}$ for visualization. All images were analyzed with Living Image ${ }^{\circledR}$ v 4.5 (PerkinElmer, USA) to obtain the fluorescence intensity in the selected region of interest (ROI) of fluorescence (flux/sec), which was selected as a fixed rectangular area around each mouse. For the control and inhibitor treatment groups, ROI (flux/sec) for each mouse was normalized by its flux measured at $0 \mathrm{hr}$. For the WT and Bcrp KO mice, ROI of the WT mice at 0 hour was used for normalizing all fluorescence (ROI) data. Normalized ROI data was used for calculation of AUC $\mathrm{FL}_{0-6 h r} \neg$ in GraphPad Prism v 5.02.

\section{Pharmacokinetic study of PhA}

Immediately after the imaging, as mice came out of anesthesia, $50 \mu \mathrm{L}$ of blood samples were collected by saphenous vein into a microvette capillary blood collection tubes (Sarstedt, obtained from Fisher Scientific, Pittsburgh, PA) and serum was separated by centrifuging the samples at $5000 \mathrm{rpm}$ for $10 \mathrm{~min}$. Blood samples were collected at 1 , 2, 4 and $6 \mathrm{hrs}$ for measurement of $\mathrm{PhA}$ concentration in serum.

\section{Measurement of $\mathrm{PhA}$ concentration in whole serum}

A fluorescence-based method was used to measure PhA concentration in whole serum. A calibration of known $\mathrm{PhA}$ concentrations was prepared by spiking known $\mathrm{PhA}$ 
concentrations prepared in DMSO into $24 \mu \mathrm{L}$ of blank serum, collected from mice on AFF diet. $25 \mu \mathrm{L}$ of standard serum concentrations and serum samples collected for the study were diluted to $100 \mu \mathrm{L}$ with distilled water in a 96 well clear bottom black polystyrene plate (Cat\# 3603, Corning, NY, USA). PhA fluorescence was measured at $400 \mathrm{~nm}$ (Ex) and $670 \mathrm{~nm}(\mathrm{Em})$ on a Synergy H4 Hybrid Reader (BioTek, Winooski, VT, USA). All fluorescence values were blank-corrected and unknown sample PhA concentrations were interpolated from a freshly prepared $\mathrm{PhA}$ standard curve $(0.02 \mu \mathrm{M}$ to $10 \mu \mathrm{M})$ and the $\mathrm{PhA}$ concentration for each mouse was used to measure AUCserum 0-6hr $\urcorner$ in GraphPad Prism v 5.02.

\section{Ex vivo fluorescence imaging of mouse intestine}

After in vivo imaging, mice were immediately euthanized and blood was collected by cardiac puncture. The small intestine was dissected out and fluorescence $(675 \mathrm{~nm}$ excitation and $840 \mathrm{~nm}$ emission) images were obtained before and after flushing out of intestinal contents with PBS (5 ml, three times). Fluorescence images were obtained using one second exposure with a filter setting for near infrared. Images were scaled to a maximum intensity of $1 \times 108$ photons $/ \mathrm{s} / \mathrm{cm} 2 / \mathrm{sr}$.

\section{Fluorescence microscopy of intestinal sections}

Intestinal segments of WT and Bcrp KO mice on a regular diet were washed with cold PBS and filled with warm 3\% agarose. Paraffin blocks were prepared from agarose filled intestines, which were precision-cut for preparing slides for fluorescence microscope imaging (Nikon ECLIPSE Ti)

\section{In vitro assay for measuring IC50 of Bcrp inhibitors}

A 96 well plate-based assay utilizing control and BCRP overexpressing MadinDerby Canine Kidney (MDCKII) cells [295], was used for measuring the IC50 of selected BCRP inhibitor drugs using PhA as a probe BCRP substrate [288]. Briefly, $1^{*} 10^{\wedge} 4$ cells/ well were plated in a 96 well plate and incubated at $37^{\circ} \mathrm{C}$ till the cells were at least $80-90 \%$ confluent. On the day of the experiment, media was removed, cells were washed with PBS and incubated for 30 min with or without BCRP inhibitors prepared in $100 \mu \mathrm{L}$ of modified Krebs Ringer buffer ( $115 \mathrm{mM} \mathrm{NaCl}, 5.9 \mathrm{mM} \mathrm{KCl}, 1.2 \mathrm{mM} \mathrm{MgCl} 2$, $1.2 \mathrm{mM} \mathrm{NaH2PO} 4,2.5 \mathrm{mM} \mathrm{CaCl} 2,2.5 \mathrm{mM} \mathrm{NaHCO} 3$ and $10 \mathrm{mM}$ Glucose) [296]. After incubation with inhibitors, media was removed and cells were incubated with $1 \mu \mathrm{M} \mathrm{PhA}$ with or without the inhibitor prepared in $100 \mu \mathrm{L}$ Krebs-Ringer buffer for $2 \mathrm{hrs}$. Fumitremorgin C (FTC) at a concentration of $5 \mu \mathrm{M}$ was used as a standard BCRP inhibitor. After 2 hrs, cells were washed with chilled Dulbecco's phosphate buffer saline (DPBS) twice, $100 \mu \mathrm{L}$ DPBS added, and fluorescence measured on Synergy H4 Hybrid Reader at $400 \mathrm{~nm}(\mathrm{Ex})$ and $670 \mathrm{~nm}(\mathrm{Em})$. Immediately after fluorescence measurement, $100 \mu \mathrm{L}$ Promega CellTiter-Glo® reagent (Fisher Scientific, Lenexa, KS, USA), was 
added to each well, incubated at $37^{\circ} \mathrm{C}$ for 5 minutes, and luminescence measured. To calculate the cell number/well 120000 MDCKII control and BCRP overexpressing cells were plated per well and diluted with cell culture media 1:1 up to 937 cells/well ( $n=6$ each dilution each cell type), incubated at $37^{\circ} \mathrm{C}$ for 6 hours for the cells to attach. After 6 hrs, cells are washed with chilled DPBS, after which $100 \mu \mathrm{L}$ DPBS and CellTiter-Glo ${ }^{\circledR}$ reagent at room temperature was added to the cells, incubated for $5 \mathrm{~min}$ at $37^{\circ} \mathrm{C}$ and luminescence measured. First, the results were background-corrected, normalized with cell number in each well to calculate $\mathrm{PhA}$ fluorescence/ $10^{\wedge} 3$ cells/ well. Fold change of $\mathrm{PhA}$ accumulation in MDCKII cells in presence of BCRP was calculated by dividing MDCKII-BCRP fluorescence/cell with that of MDCKII. The \% inhibition by BCRP inhibitors was calculated by the following equation:

$$
\% \text { Inhibition }=\frac{(\text { Fluorescence Inhibitor }- \text { Fluorescence no inhibitor })}{(\text { Fluorescence } \mathrm{FTC}-\text { Fluorescence no inhibitor })} * 100
$$

IC50 values of the inhibitors were calculated using GraphPad prism v5.02. All assays were run at least three times with $n=6$ wells per inhibitor or standard inhibitor concentration in each assay. Results of all experiments were combined $(n=18)$ to calculate final parameters.

\section{Statistical analysis}

All serum PhA concentration-time data were plotted as mean PhA concentration \pm SEM and similarly, the whole-body fluorescence data over zero-hour of each individual mouse (for the inhibitor study), or over WT mice zero-hour fluorescence (for Bcrp KO study), were plotted as mean ratio \pm SEM. GraphPad Prism v5.02 was used to calculate the area under the concentration - time or fluorescence (flux/sec) ratio - time curve for individual mice up to 6 hours (AUCserum 0-6h or AUCFL 0-6h). Mean AUC \pm SEM are plotted and compared with the control or WT using the unpaired t-test, significance is calculated at $\mathrm{p}<0.05$. All calculations were done on GraphPad Prism v5.02.

\section{Results}

\section{Bcrp KO mice on a regular diet have higher whole-body fluorescence, arising from PhA, than WT mice}

It has been previously reported that whole body autofluorescence during live mice imaging can be reduced by feeding the mice alfalfa free (AFF) diet, and also by choosing an excitation wavelength at the near infrared region (NIR, $700 \mathrm{~nm}$ to $1 \mathrm{~mm}$ ) of light [297, 298]. To determine differences in autofluorescence, we imaged both WT and Bcrp KO mice and found a higher whole-body autofluorescence in the Bcrp KO compared to the WT mice, when the mice were fed a regular diet (Figure 4-1A). Alfalfa is known to be a source of chlorophyll, and its catabolite pheophorbide $\mathrm{A}(\mathrm{PhA})$, which is a known BCRP substrate and has fluorescence excitation and emission in the NIR region. Therefore, to 


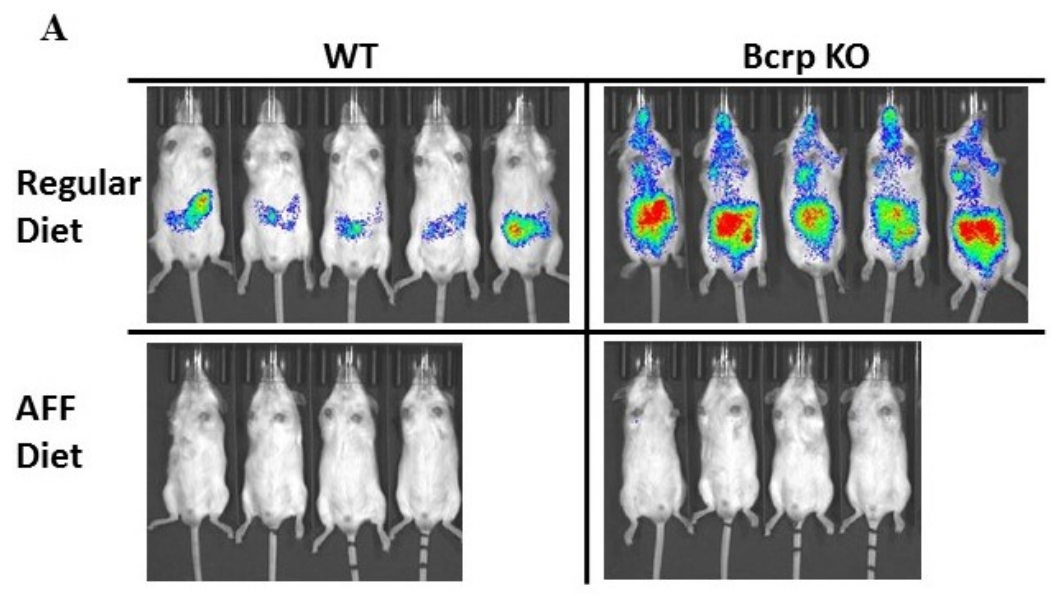

B

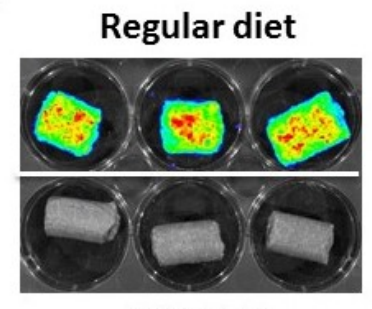

AFF diet

C

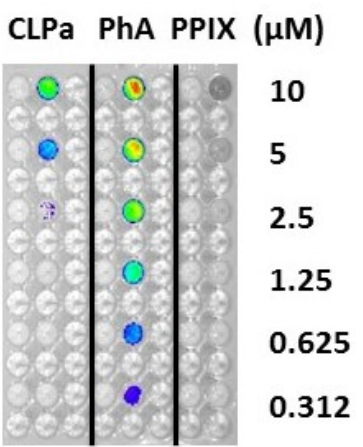

Figure 4-1. Autofluorescence derived from dietary $\mathrm{PhA}$ is higher in the Bcrp KO compared to WT mice

Note: Comparison of fluorescence (Ex675/Em840) in WT and Bcrp KO mice fed a regular diet or AFF diet at least for three days (A). Comparison of fluorescence (Ex675/Em840) in a regular and AFF diet (B). Fluorescence comparison of known concentrations of chlorophyll a (CLPa), PhA and a prototypical Bcrp substrate protoporphyrin IX (PPIX) fluorescence with filter setting (Ex675/Em840) (C). 
determine the contribution of $\mathrm{PhA}$ to the observed autofluorescence, we imaged both WT and Bcrp KO mice after feeding them an alfalfa free (AFF) diet. We observed a total loss of whole-body autofluorescence in both WT and Bcrp KO mice (Figure 4-1A). To confirm if diet was the source of the observed fluorescence, using the same imaging parameters, we compared the regular and AFF diet pellets and observed fluorescence only from the regular food pellets (Figure 4-1B). To further confirm the source of fluorescence as dietary $\mathrm{PhA}$, we compared $\mathrm{PhA}$, chlorophyll a (CLPa) and another fluorescent Bcrp endogenous substrate protoporphyrin IX (PPIX) at a concentration range of 0.31 to $10 \mu \mathrm{M}$, at the same fluorescence setting (excitation at $670 \mathrm{~nm}$ and emission at $840 \mathrm{~nm}$ ). We found that only PhA can be detected at $0.31 \mu \mathrm{M}$, which is almost 10 -fold lower than the lowest detectable concentration of CLPa $(2.5 \mu \mathrm{M})$, and that PhA had a higher fluorescent intensity then CLPa at each concentration (Figure 4-1C).

\section{Pheophorbide A is present in regular mouse diet, but not in AFF diet}

To further verify that the source of fluorescence from regular diet is $\mathrm{PhA}$, and that its absence reduces whole-body fluorescence in mice, we analyzed the amount of PhA in mouse food pellets using an HPLC-RF- based method (Figure 4-2). We observed that $\mathrm{PhA}$ is present only in the standard (Figure 4-2A) and the regular food pellet extract (Figure 4-2B), but not in the AFF pellet extract (Figure 4-2C). Using a calibration of known concentrations of $\mathrm{PhA}$, we found the $\mathrm{PhA}$ concentration in the regular diet to be $1.71 \mu \mathrm{g} / \mathrm{gm}$ of food pellet. Assuming daily food intake for a $30 \mathrm{gm}$ mouse is $6 \mathrm{gms}$, it can be estimated that a mouse on a regular diet consumes $343 \mu \mathrm{g} \mathrm{PhA} / \mathrm{kg}$ body weight daily.

\section{Bcrp gene deletion leads to higher absorption of $\mathrm{PhA}$ in mouse enterocytes}

During in vivo live animal imaging, we observed the maximum fluorescence in the intestinal region of Bcrp KO mice (Figure 4-1A). This was unanticipated since we expected a higher level of PhA absorbance in the Bcrp KO mice. Therefore, to determine if this fluorescence was due to trapping of $\mathrm{PhA}$ in the intestinal enterocytes we analyzed the localization of fluorescence in WT and Bcrp KO mice fed a regular diet, by ex vivo imaging of isolated intestine (Figure 4-3A) and fluorescence microscopy of intestinal sections (Figure 4-3B). A high fluorescence intensity was observed in the upper intestinal segment of both WT and Bcrp KO mice (data not shown). But after washing and removal of food contents, the upper intestinal segment of Bcrp KO mice displayed considerably higher fluorescence, compared to that in WT mice (Figure 4-3A). Fluorescence microscopy of transverse sections of a paraffin fixed intestinal segment showed higher fluorescence in the enterocytes of the Bcrp KO mice, compared to the WT (Figure 4-3B), confirming that the absence of Bcrp at the enterocytes may lead to higher $\mathrm{PhA}$ absorption in the Bcrp KO mice fed a regular diet. 
A

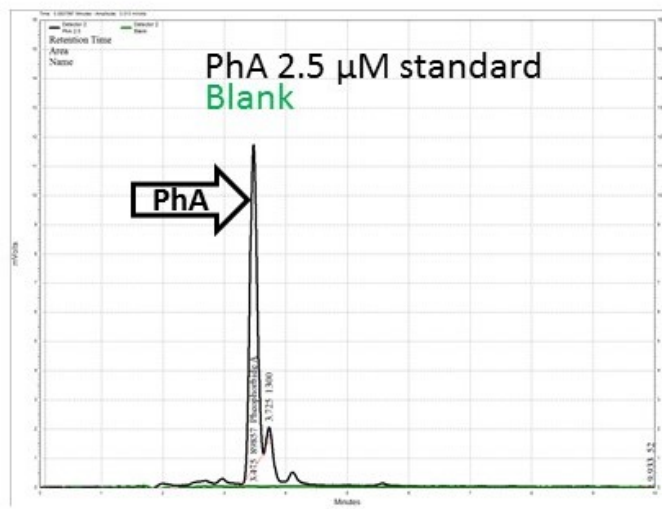

C

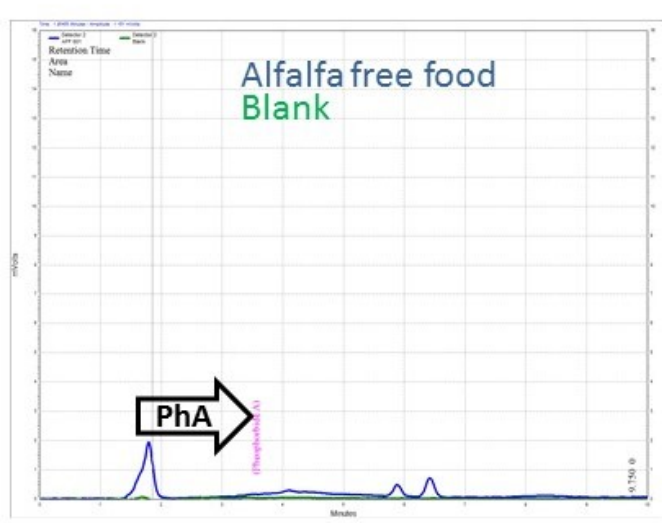

B

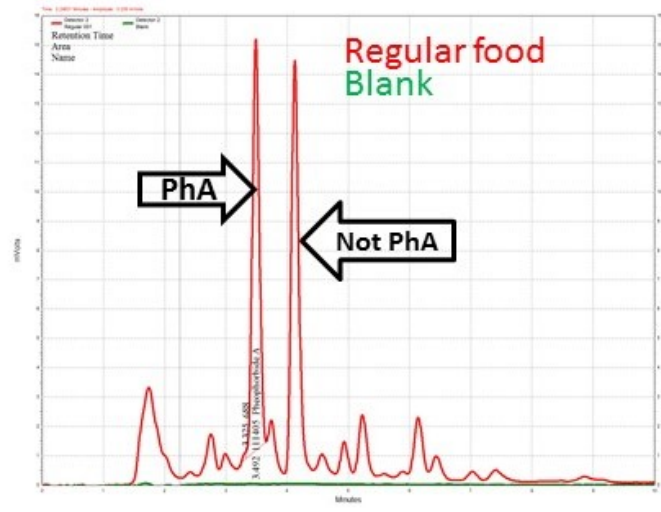

$400 \mathrm{~nm}(\mathrm{Ex})$ $670 \mathrm{~nm}(\mathrm{Em})$

Figure 4-2. PhA is present in the regular mouse diet not in the AFF diet

Notes: Representative HPLC-RF run profile of $2.5 \mu \mathrm{M}$ known concentration of PhA (A), methanol extract from regular rodent diet (B), and the alfalfa free (AFF) defined diet (C). $\mathrm{PhA}$ peak or peak position is indicated in each graph. 


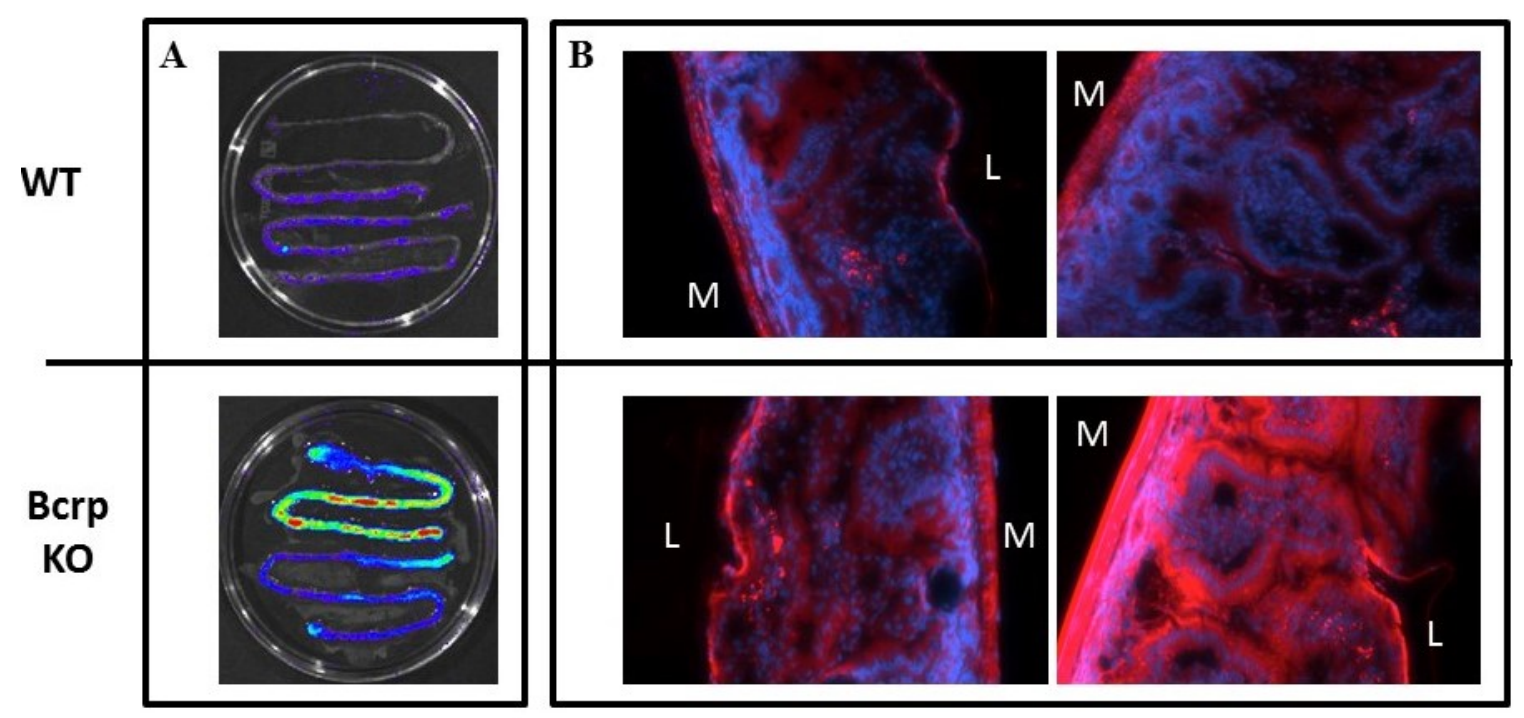

L: Luminal side; M: Blood side (Circular muscle)

Figure 4-3. Absence of Bcrp leads to higher absorption of PhA in enterocytes

Notes: Ex vivo image comparison of intestinal segments of WT and Bcrp KO mice on a regular diet, after flushing out the gut contents with saline (A). Microscopic examination of cross-sectional images of upper intestine from WT and Bcrp KO mice on regular diet; blue is Dapi stain for nuclear localization) and red is Pheophorbide A using the Cyanine5 Ex/Em wavelengths (B). 


\section{Oral absorption of PhA is greater in Bcrp KO mice treated with exogenous PhA}

To eliminate background fluorescence and increase the uniformity and amount of PhA exposure, WT and Bcrp KO mice were fed an AFF diet for 3 days, then administered $\mathrm{PhA}$ orally at a dose of $10 \mathrm{mg} / \mathrm{kg}$ and fasted for $6 \mathrm{hrs}$. Representative images at $2 \mathrm{hrs}$ after $\mathrm{PhA}$ dosing displayed significantly greater whole-body fluorescence in the Bcrp KO compared to WT mice, as shown in Figure 4-4A. The whole-body fluorescence at different time-points, normalized to the fluorescence in WT mice at baseline, when plotted against time after dosing, exhibited significantly higher fluorescence in Bcrp KO mice across all observed time points (1, 2, 4 and 6 hrs) (Figure 4-4B). Quantification of the area under fluorescence ratio time curve up to 6 hrs (AUCFL 0-6h) showed a significant increase (3.5-fold) in fluorescence in the Bcrp KO compared to WT mice (Figure 4-4C). We also measured the serum concentration of $\mathrm{PhA}$ in the same experiment at 1, 2, 4 and 6 hrs. The serum concentration of PhA in the Bcrp KO mice was also found to be significantly higher across all observed time points compared to WT mice (Figure 4-4D) with a 50 fold increase in area under concentration-time curve calculated up to 6 hrs (AUCserum 0-6h) (Figure 4-4E). These results, showing a significantly higher fold increase of $\mathrm{PhA}$ fluorescence, both whole-body and serum, in Bcrp KO mice compared to WT, suggest that orally administered $\mathrm{PhA}$ can be utilized to capture a range of inhibition by Bcrp inhibitors, and thereby identify Bcrp-mediated drug-drug interactions (DDIs) in mice.

\section{Selected Bcrp inhibitors exhibited comparable in vitro IC50 values with PhA as reported previously}

To further test our hypothesis that $\mathrm{PhA}$ can be used as an in vivo probe for identification of intestinal Bcrp-mediated DDIs, we selected known Bcrp inhibitors as per FDA guidance based on their [(Clinical dose/250 ml)/IC50 or Igut/IC50] (Table 4-1), where Igut is the maximum human dose in the apparent human gut fluid volume of 250 $\mathrm{ml}$. While IC50 values of the selected inhibitors (except dasatinib) were reported in the literature, a wide variety of Bcrp probe substrates as well as cell lines were used for these IC50 measurements. Using PhA as a BCRP probe substrate, we used MDCKII-BCRP cell-based assays to determine the IC50 of the selected BCRP inhibitors (Figure 4-5). Lapatinib (LPB) was found to be the strongest BCRP inhibitor with a measured IC50 of $40 \mathrm{nM}$, followed by elacridar (ECD) $(0.21 \mu \mathrm{M})$, sorafenib (SFB) $(2.18 \mu \mathrm{M})$, curcumin (CCM) $(2.58 \mu \mathrm{M})$, pantoprazole (PPZ) $(11.19 \mu \mathrm{M})$ and dasatinib (DSB) $(22.76 \mu \mathrm{M})$. The IC50 of these inhibitors were found to be similar to those reported in the literature using different BCRP probe substrates (Table 4-1), except for PPZ, for which the observed IC50 was 2-fold higher than the reported IC50 $(5.5 \mu \mathrm{M})$. Assuming a $0.8 \mathrm{~mL}$ mouse stomach volume, $\mathrm{C}_{\text {gut }} / \mathrm{IC} 50$ was $\geq 10$ for all the inhibitors (Table 4-1), indicating a possible drug-drug interaction observation with orally dosed BCRP substrates in mice, where, Cgut is the selected inhibitor dose $/ 0.8 \mathrm{~mL}$. 
Whole Body

A
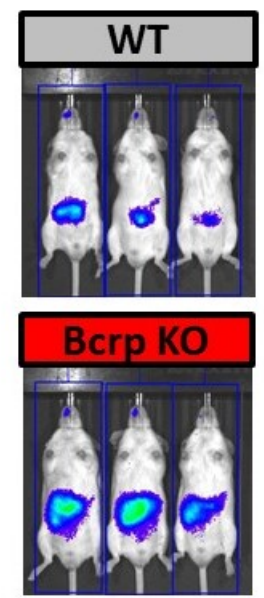

B

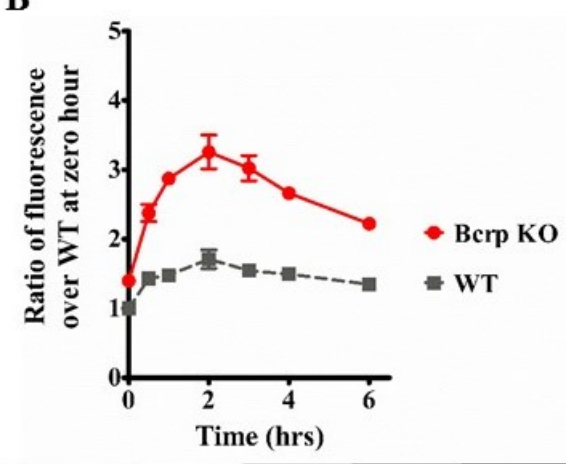

C

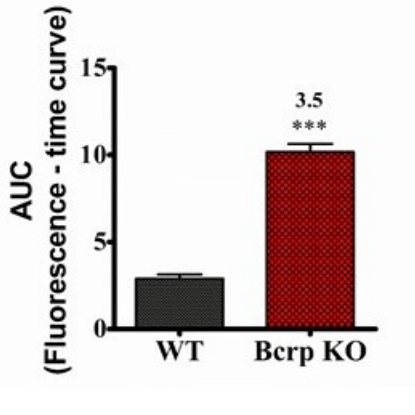

Serum

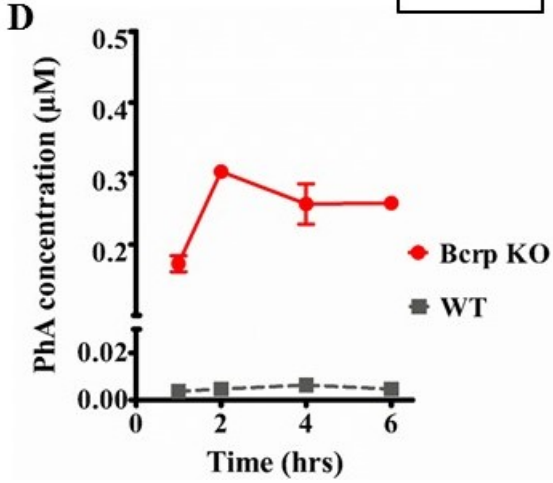

$\mathbf{E}$

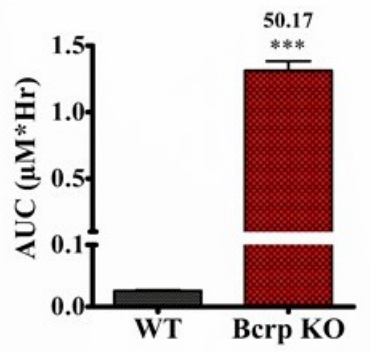

Figure 4-4. PhA oral absorption is significantly higher in the Bcrp KO mice

Notes: Representative image comparing of PhA fluorescence (Ex675/Em840) in WT and Bcrp KO mice, $2 \mathrm{hrs}$ after oral administration of $10 \mathrm{mg} / \mathrm{kg} \mathrm{PhA}$ (A). Fluorescence intensity over zero- hour WT fluorescence-time profile up to 6 hours following $10 \mathrm{mg} / \mathrm{kg}$ of $\mathrm{PhA}$ oral administration in Bcrp WT and $\mathrm{KO}$ mice $(\mathrm{n}=3)(\mathbf{B})$. Comparison of Area under the fluorescence ratio time curve up to 6 hrs (AUC $\mathrm{FL}_{\mathrm{FL}}$-6h) in Figure B calculated using GraphPad Prism v5.02 (C). Comparison of PhA serum concentration at 1, 2,4 and 6 hrs from the WT and Bcrp KO mice dosed $10 \mathrm{mg} / \mathrm{kg} \mathrm{PhA}$ (D) and the associated $\mathrm{AUC}_{\text {Serum } 0-6 \mathrm{~h}}(\mathbf{E})$. Results are presented as means $\pm \mathrm{SEM}$; and significance is calculated at $\mathrm{p}<0.05$ by comparing the means with unpaired t-test. $* * * \mathrm{P}<0.001$. 
Table 4-1. Results of all in vitro, in vivo experiments and literature data-based comparisons

\begin{tabular}{|c|c|c|c|c|c|c|c|}
\hline Details & Parameters (Units) & DSB & SFB & PPZ & ECD & CCM & LPB \\
\hline \multirow{4}{*}{$\begin{array}{l}\text { Known } \\
\text { parameters from } \\
\text { literature and } \\
\text { database }\end{array}$} & Mol. Wt. (gm/mol) & 488.01 & 464.83 & 383.37 & 563.65 & 368.38 & 581.06 \\
\hline & $\begin{array}{l}\text { Pred. Sol. }(\mu \mathrm{M}) \\
\text { (DrugBank) }\end{array}$ & 26.23 & 3.68 & 1291.18 & 4.99 & 15.61 & 38.38 \\
\hline & $\begin{array}{l}\text { Pred. } \log P \\
\text { (DrugBank) }\end{array}$ & 2.77 & 4.12 & 2.11 & 6.81 & 3.62 & 5.18 \\
\hline & Reported IC50 $(\mu \mathrm{M})$ & & $3.1^{1}$ & $5.5^{2}$ & $0.31^{3}$ & $1.6^{4}$ & $0.025^{5}$ \\
\hline \multirow[t]{5}{*}{$\begin{array}{l}\text { Clinical } \\
\text { information }\end{array}$} & Dose (mg) & & $\begin{array}{l}400 \mathrm{mg} \\
\text { BID }\end{array}$ & $\begin{array}{l}40 \mathrm{mg} \\
\mathrm{SD}^{6}\end{array}$ & $\begin{array}{c}400 \mathrm{mg} \\
\mathrm{BID}^{6}\end{array}$ & $\begin{array}{l}2 \mathrm{gm}^{6} \\
\mathrm{SD}^{6}\end{array}$ & $\underset{6}{1250} \mathrm{QD}$ \\
\hline & $\mathrm{C}_{\max }(\mu \mathrm{M})$ & & $6.45^{7}$ & $6.5^{6}$ & $0.327^{6}$ & & $4.2^{6}$ \\
\hline & $\mathrm{I}_{\mathrm{gut}} / \mathrm{IC} 50$ & & 1110 & $67^{6}$ & $9200^{6}$ & $14000^{6}$ & $210000^{6}$ \\
\hline & $\mathrm{C}_{\max } / \mathrm{IC} 50$ & & 2.08 & $1.2^{6}$ & $1.1^{6}$ & & $170^{6}$ \\
\hline & Possible DDI & No & Yes & Yes & Yes & Yes & Yes \\
\hline \multirow{4}{*}{$\begin{array}{l}\text { PK information } \\
\text { from mice }\end{array}$} & Dose $(\mathrm{mg} / \mathrm{kg})$ & 10 & 40 & 40 & 100 & 300 & 90 \\
\hline & $\mathrm{C}_{\max }(\mu \mathrm{M})$ & $\sim 0.7^{10}$ & $21^{1}$ & & $0.78^{8}$ & $\sim 0.06^{12}$ & $\sim 10^{11}$ \\
\hline & $\mathrm{T}_{\max }(\mathrm{Hr})$ & $0.25-2$ & $1^{9}$ & & 8 & 1.5 & $0.5-1$ \\
\hline & $\mathrm{t} 1 / 2(\mathrm{Hr})$ & & & & 20 & 12 & 3 \\
\hline \multirow{7}{*}{$\begin{array}{l}\text { Prediction of } \\
\text { possible outcome } \\
\text { in mouse based on } \\
\text { selected dose and } \\
\text { observed IC50 }\end{array}$} & Observed IC50 $(\mu \mathrm{M})$ & 22.76 & 2.18 & 11.19 & 0.21 & 2.52 & 0.04 \\
\hline & $\mathrm{C}_{\max } / \mathrm{IC} 50$ & 0.03 & 9.63 & & 3.71 & 0.02 & 250 \\
\hline & $\begin{array}{l}\text { Oral Dose for mice } \\
\text { study }(\mathrm{mg} / \mathrm{kg})\end{array}$ & 10 & 40 & 40 & 100 & 300 & 90 \\
\hline & $\mathrm{C}_{\text {gut }}(\mu \mathrm{M})$ & 639 & 2689 & 3260 & 5544 & 25449 & 4840 \\
\hline & $\mathrm{C}_{\text {gut }} / \mathrm{IC} 50$ & 28 & 1233 & 291 & 26400 & 10098 & 121000 \\
\hline & $\mathrm{C}_{\text {gut_soluble }} / \mathrm{IC} 50$ & 1.2 & 1.7 & 115.4 & 23.7 & 6.2 & 959.5 \\
\hline & Expected Oral DDI & No & May be & Possible & Yes & Yes & Yes \\
\hline \multirow{3}{*}{$\begin{array}{l}\text { In vivo serum } \\
\text { concentration } \\
\text { result }\end{array}$} & $\begin{array}{l}\mathrm{AUC}_{\text {Serum }} 0-6 \mathrm{~h} \\
\left(\mu \mathrm{M}^{*} \mathrm{Hr}\right)\end{array}$ & $\begin{array}{c}0.11 \pm \\
0.002\end{array}$ & $\begin{array}{c}0.13 \pm \\
0.02\end{array}$ & $\begin{array}{c}0.18 \pm \\
0.01\end{array}$ & $\begin{array}{c}0.46 \pm \\
0.08\end{array}$ & $\begin{array}{c}0.70 \pm \\
0.1\end{array}$ & $\begin{array}{c}1.24 \pm \\
0.09\end{array}$ \\
\hline & $\begin{array}{l}\text { Fold Change over } \\
\text { control }\end{array}$ & 1.24 & 1.45 & 2.02 & 5.17 & 7.79 & 13.78 \\
\hline & Observed DDI? & No & May be & Yes & Yes & Yes & Yes \\
\hline \multirow{3}{*}{$\begin{array}{l}\text { Live animal } \\
\text { fluorescence } \\
\text { result }\end{array}$} & $\mathrm{AUC}_{\mathrm{FL} 0-6 \mathrm{~h}}$ & $\begin{array}{c}5.82 \pm \\
1.27\end{array}$ & $\begin{array}{c}6.98 \pm \\
1.15\end{array}$ & $\begin{array}{c}4.07 \pm \\
0.71\end{array}$ & $\begin{array}{c}10.55 \pm \\
2.12\end{array}$ & $\begin{array}{c}11.2 \pm \\
1.75\end{array}$ & $\begin{array}{c}11.66 \pm \\
1.54\end{array}$ \\
\hline & $\begin{array}{l}\text { Fold Change over } \\
\text { control }\end{array}$ & 1.2 & 1.44 & 0.84 & 2.17 & 2.31 & 2.4 \\
\hline & Observed DDI? & No & May be & No & Yes & Yes & Yes \\
\hline
\end{tabular}

Notes: Mol. Wt. $=$ molecular weight; Pred. Sol $=$ Predicted aqueous solubility; Igut $=$ Clinical dose $/ 250 \mathrm{~mL}$; $\mathrm{C}_{\text {gut }}=$ mouse dose $/ 0.8 \mathrm{~mL}$ (mouse gut fluid volume); Cgut_soluble $_{\text {e }}$ Pred. Solubility ${ }^{12}[312]$

${ }^{1}[166] ;{ }^{2}[309] ;{ }^{3}[310] ;{ }^{4}[259] ;{ }^{5}[134] ;{ }^{6}[71] ;{ }^{7}[305] ;{ }^{8}[269] ;{ }^{9}[94] ;{ }^{10}[308] ;{ }^{11}[311] ;$ 

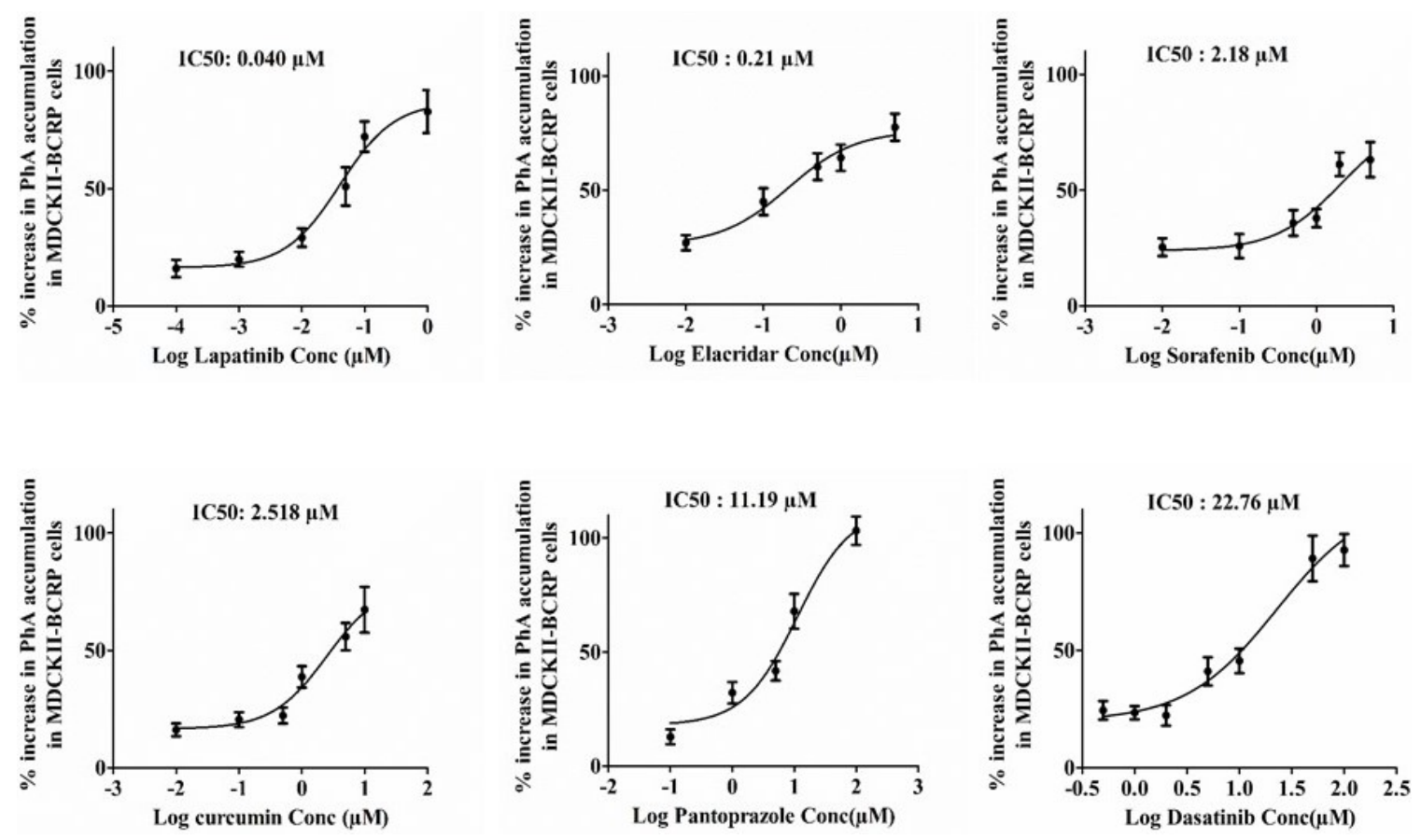

Figure 4-5. Determination of IC50 for selected Bcrp inhibitors using PhA as a probe substrate

Notes: Mean \% increase in Pheophorbide A accumulation \pm SEM in the MDCKII cells overexpressing BCRP in presence of selected Bcrp inhibitors was plotted against log transformed inhibitor concentration. IC50 was determined using GraphPad Prism v5.02. 


\section{Bcrp inhibitors increase whole body and serum fluorescence of $\mathrm{PhA}$}

Based on reported literature, the selected drugs were expected to cause drug-drug interactions with Bcrp substrates when administered concomitantly, via an oral route, and increase systemic exposure (blood/ plasma / serum AUC) of the substrate. Therefore, we dosed the inhibitors orally, along with the Bcrp probe substrate $\mathrm{PhA}$, and measured whole body fluorescence and serum $\mathrm{PhA}$ concentrations. Whole-body fluorescence imaging in mice treated with the Bcrp inhibitors LPB, ECD and CCM displayed higher fluorescence compared to the control-treated mice (Figure 4-6). After quantification of fluorescence flux/second and normalization with baseline fluorescence from the same mice, these values were plotted against time after dosing (Figure 4-7A and B), and AUCFL 0-6h was calculated for all the inhibitor-treated and control mice. LPB exhibited the maximum fold increase (2.4-fold) of AUCFL 0-6h over control mice, followed by CCM (2.3-fold), ECD (2.2-fold) and SFB (1.44-fold) (Figure 4-7C). PPZ showed a decrease in AUCFL 0-6h compared to control (0.84-fold) and DSB did not significantly increase the AUCFL 0-6h over control mice.

Serum fluorescence spectra of $\mathrm{PhA}$ was measured and compared with a standard $\mathrm{PhA}$ sample to confirm the identity of $\mathrm{PhA}$ in each sample. Representative $\mathrm{PhA}$ spectra of control (CT) mice, curcumin (CCM) and lapatinib (LPB) treated mice are showing $\mathrm{PhA}$ peak at $670 \mathrm{~nm}$ in each sample corresponding to the standard $\mathrm{PhA}$ peak (Figure 4-8).

The PhA serum concentration-time plots after treatment with Bcrp inhibitors displayed a similar trend as observed with whole body fluorescence (Figure 4-7D and E), with a similar rank order of inhibitor-mediated increase in AUCserum 0-6h observed as was

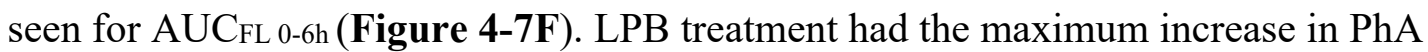
fluorescence (13.8-fold), followed by CCM (7.79-fold), ECD (5.2-fold), PPZ (2.02-fold) and SFB (1.45-fold). No significant effect of DSB was found on serum PhA concentration consistent with the lack of effect on PhA whole-body fluorescence. PPZ showed a significant increase in serum PhA AUCSerum 0-6h, but not AUCFL 0-6h, measured by the whole-body fluorescence data. Whereas $\mathrm{C}_{\text {gut }} / \mathrm{IC} 50$ for all inhibitors were

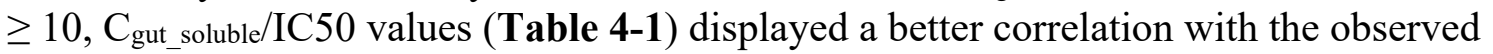
fold change by Bcrp inhibitors, where Cgut_soluble is the predicted solubility of the inhibitors obtained from DrugBank database (https://www.drugbank.ca).

\section{Discussion}

The ATP-binding efflux transporter Bcrp plays a significant role in controlling xenobiotic exposure by reducing absorption and increasing elimination of its substrates [129]. Drug-drug interactions (DDI) associated with Bcrp have been reported for Bcrp substrates such as topotecan [299], sulfasalazine [259] and rosuvastatin [300]. Based on the clinical evidence and importance of its transporter effect, Bcrp has been recognized by FDA and ITC as having the potential for causing clinical DDI $[1,25,80]$. Due to overlapping substrates and inhibitors of drug transporters as well as metabolizing 


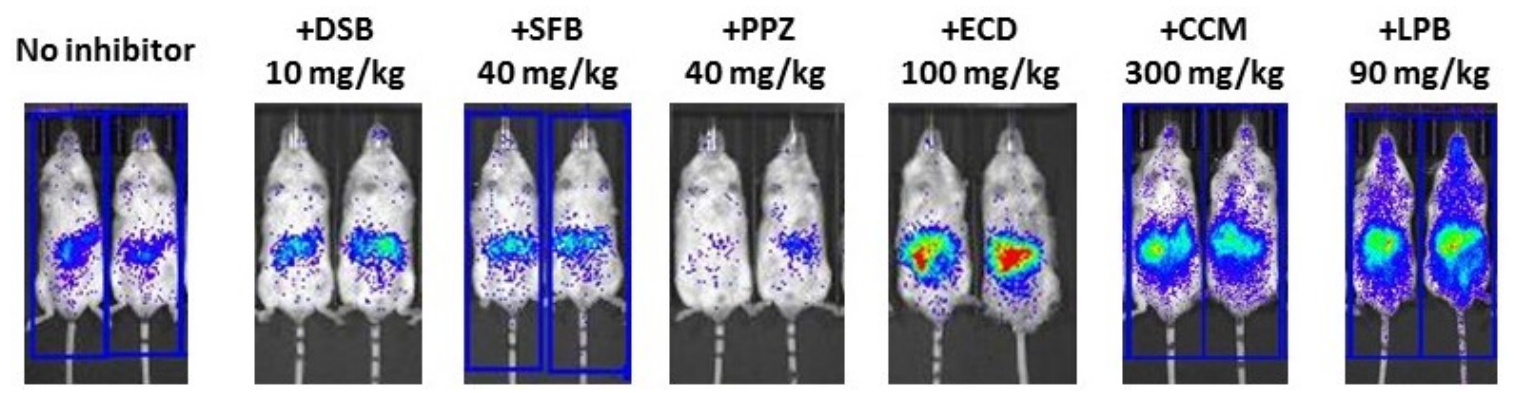

Figure 4-6. Inhibition of BCRP increased whole body fluorescence in control FVB mice orally dosed with $\mathrm{PhA}$

Note: Six hour representative image comparing fluorescence (Ex675/Em840) in control mice orally administrated $10 \mathrm{mg} / \mathrm{kg}$ of $\mathrm{PhA} \pm$ Bcrp inhibitors, $1 \mathrm{hr}$ prior to $\mathrm{PhA}$ administration as indicated in the method. 


\section{Whole Body}

A

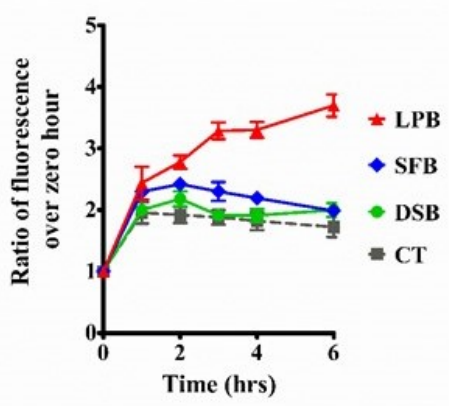

B

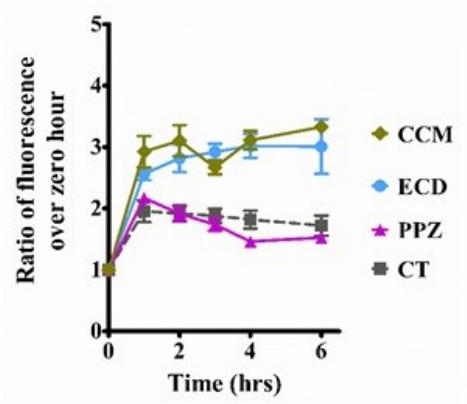

C

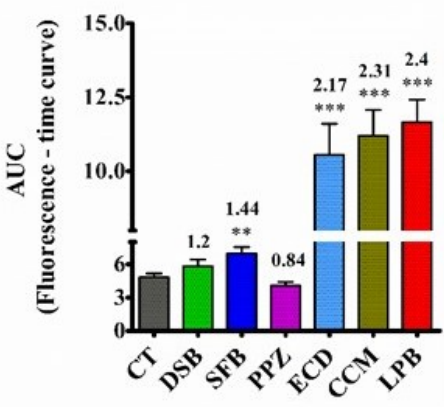

Serum

D

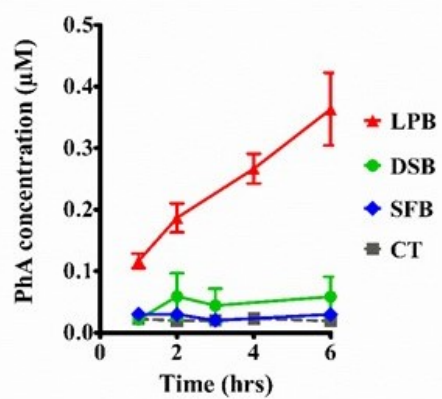

E

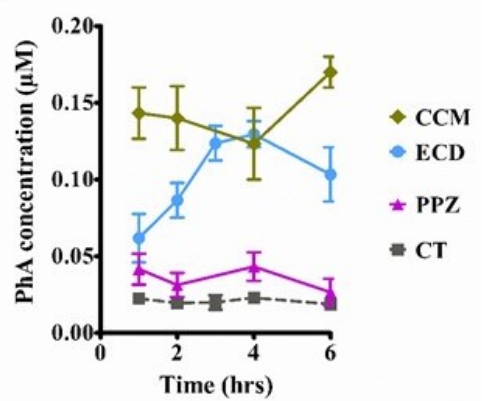

$\mathbf{F}$

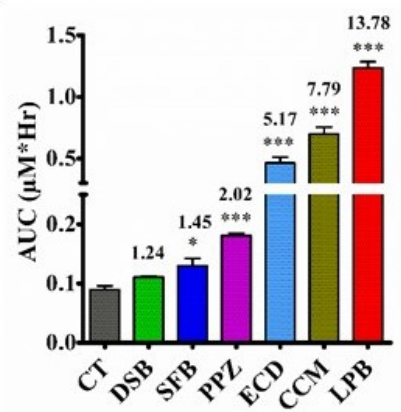

Figure 4-7. Inhibition of BCRP increased whole body fluorescence as well as the serum concentration of $\mathrm{PhA}$ in control FVB mice

Notes: Whole body fluorescence (flux/sec) was normalized to the zero-hour fluorescence of the same mice and data plotted as mean fluorescence ratio $\pm \mathrm{SEM}$ vs. time (hrs), for the tyrosine kinase inhibitors (A) and other inhibitors (B). AUC $\mathrm{FL}_{0-6 \mathrm{~h}}$ calculated for each mouse and plotted as mean \pm SEM (C). PhA serum concentration- time plot for the control and tyrosine kinase inhibitors (D) and other inhibitors (E) plotted as mean \pm SEM and the associated AUCSerum 0-6h calculated using GraphPad Prism v5.02 (F). Significance is calculated by comparing the mean AUC after inhibitor treatment with that of control AUC by unpaired t-test. Significance is calculated at $\mathrm{p}<0.05$. ${ }^{*} \mathrm{p}<0.05,{ }^{*} \mathrm{p}<0.01$, $* * * \mathrm{p}<0.001$. 

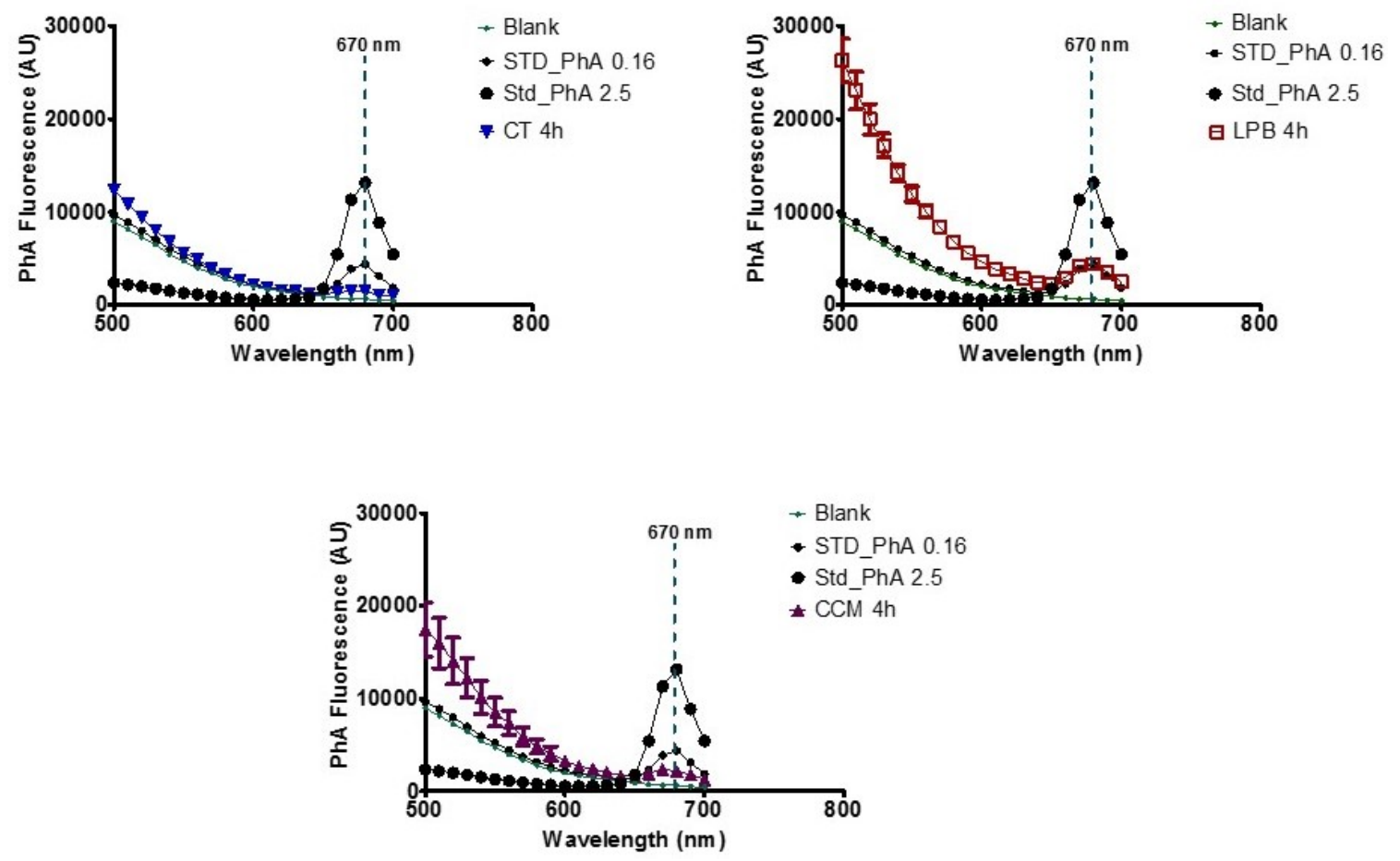

Figure 4-8. Fluorescence spectra showing identity of the $\mathrm{PhA}$ peak used for determination of $\mathrm{PhA}$ concentration in serum

Note: Mean fluorescence \pm SD spectrum with excitation at $400 \mathrm{~nm}$ and emission at 670 $\mathrm{nm}$ for the PhA standard prepared in serum, control serum from PhA (10 mg/kg) dosed mice and inhibitor (LPB and CCM) treated mice. 
enzymes, interpretation of clinical DDI studies utilizing probe substrates is often difficult. Therefore, a more selective or specific probe-based assay, or utilization of a biomarker for elucidation of Bcrp-mediated DDI is highly desirable.

PhA has been documented to be a substrate of human BCRP in cell lines [288] and we now show it is a substrate for mouse Bcrp as well (Figure 4-3). Mouse Abcg2/Bcrp has 81\% sequence homology with human ABCG2/BCRP [55], and there is overlapping substrate and inhibitor specificity of human and murine ABCG2 [301]. Hence, the substrate and inhibitor profile of BCRP in humans should be comparable to that in mice. Bcrp KO mice of FVB background [92] are an established model for identification of clinical Bcrp substrates and inhibitors. Therefore, our experimental findings of $\mathrm{PhA}$ absorption in WT and Bcrp KO mice can likely be extrapolated to humans as well.

Using a LC-MS/MS based analytical system to compare the plasma of WT and Bcrp-Pgp double knockout ( $\mathrm{dKO}$ ) rats, we detected Pheophorbide A (PhA) only in the plasma of the dKO rats, but not in any WT rat plasma (Chapter 2). This observation, along with reports that $\mathrm{PhA}$ is a selective Bcrp dietary substrate [92, 288], prompted us to further investigate the possibility of utilizing $\mathrm{PhA}$ as an in vivo probe for identification of DDI associated with Bcrp. By utilizing an HPLC-Fluorescence Detector based analytical method, we determined PhA content in normal rodent chow to be $1.7 \mu \mathrm{g} / \mathrm{gm}$ of regular rodent food pellet (Figure 4-2). PhA is a known catabolite of chlorophyll [287], present in regular human diet. For example, chlorophyll content in 1 cup of spinach is $24 \mathrm{mg}, 1 / 2$ cup of parsley is $19 \mathrm{mg}$ and 1 cup of sugar snap peas is $24 \mathrm{mg}$ (https://draxe.com/chlorophyll-benefits/). It has also been previously reported that the plant monooxygenase enzyme can catabolize $\mathrm{PhA}$ formation from chlorophyll, thus $\mathrm{PhA}$ is present in the regular diet in human $[302,303]$. Therefore, complete absence of $\mathrm{PhA}$ in the plasma of WT rats, fed a regular diet, creates the possibility of utilizing PhA as a dietary marker of Bcrp transporter function. Also, since $\mathrm{PhA}$ is present in regular diet, it poses a phototoxicity risk due to unwanted exposure to PhA from food when taken with a Bcrp inhibitor, like pantoprazole.

PhA emits fluorescence at the NIR wavelength, thereby creating the exciting possibility of utilizing $\mathrm{PhA}$ as a probe for in vivo live animal imaging. Whole body autofluorescence in rodents has been previously reported to be affected by chlorophyll content of food and alfalfa free (AFF) diet [287]. Using whole animal fluorescence imaging at $670 \mathrm{~nm}(\mathrm{Ex})$ and $840 \mathrm{~nm}(\mathrm{Em})$, that corresponded to $\mathrm{PhA}$ fluorescence (Figure 4-1C), we observed a higher whole-body fluorescence in the Bcrp KO mice, compared to the WT mice (Figure 4-1A). Based on lack of background autofluorescence when mice are on AFF diet (Figure 4-1A), along with complete absence of PhA in the AFF diet (Figure 4-2C), we decided to feed mice an AFF diet for at least three days for the majority of our studies to eliminate the effect of background fluorescence. In addition, regular food pellet extract, as analyzed by the HPLC-RF based method, indicated the presence of an additional peak with similar fluorescence intensity as $\mathrm{PhA}$ (Figure 4-2B). Therefore, we administered mice fed an AFF diet an oral gavage of 10 $\mathrm{mg} / \mathrm{kg} \mathrm{PhA}$, to test the hypothesis that $\mathrm{PhA}$ can be used as an in vivo probe for Bcrp 
function and for screening Bcrp inhibitors for oral DDI. Pheophorbide A has already been reported to be used at a dose of $30 \mathrm{mg} / \mathrm{kg}$ intraperitoneally, or $10 \mathrm{mg} / \mathrm{kg}$ intravenously, for photodynamic therapy of oral squamous cell carcinoma (OSCC) in mice [304]. Chlorophyll, the catabolic precursor of $\mathrm{PhA}$, is also present in regular diet in humans, as discussed above. Therefore, our current findings utilizing the $10 \mathrm{mg} / \mathrm{kg} \mathrm{PhA}$ oral dose in mice may have a possible significant human translational significance.

We observed a difference in $\mathrm{PhA}$ excitation and emission wavelengths in vivo in mice $(670 \mathrm{~nm}(\mathrm{Ex})$ and $840 \mathrm{~nm}(\mathrm{Em}))$ vs. in vitro (400-410 $\mathrm{nm}(\mathrm{Ex})$ and $670 \mathrm{~nm}(\mathrm{Em}))$. This is not unexpected because live animal fluorescence is affected by a significant amount of absorbance and scattering of light due to the presence of a complex environment comprised of plasma proteins and tissue barriers [298]. This is critical since selecting the Ex/Em wavelength parameters based on in vitro $\mathrm{PhA}$ fluorescence would have resulted in overlooking its fluorescence in vivo.

High fluorescence in the intestinal region, but not systemically, in WT mice is due to $\mathrm{PhA}$ in the luminal contents. PhA is a low solubility and low permeability compound, and as a Bcrp substrate is poorly absorbed. Indeed, PhA has been reported to be mainly cleared unabsorbed in feces [287]. Interestingly, ex vivo fluorescence comparison of the intestinal segments of WT mice fed a normal PhA-containing diet, after flushing out intestinal contents, revealed a lower fluorescence in the duodenum compared to higher fluorescence in the jejunum and ileum (i.e., there is lower Bcrp expression in jejunum and ileum and hence a small amount of PhA is trapped in the lower small intestine of WT mice) (Figure 4-3A) and is consistent with the gradient of Bcrp expression reported in rodent intestine (higher in the duodenum than in the ileum), and being the lowest in the colon [105]. The increased fluorescence in the intestinal region in Bcrp KO mice (Figure 4-3A), in spite of increased systemic absorption from the lumen, was due to trapping of $\mathrm{PhA}$ in the enterocytes in the absence of Bcrp mediated efflux (Figure 4-3B).

We found a higher PhA whole body fluorescence in the Bcrp KO compared to WT mice, leading to a significantly higher (3.5-fold) area under the fluorescence ratio time curve up to 6 hrs (AUCFL 0-6h) (Figure 4-4B and C). Comparing the serum PhA concentration in the same experiment, we observed a 50-fold increase in $\mathrm{PhA}$ area under serum concentration - time curve up to 6 hours (AUCserum 0-6h) in the Bcrp KO compared to WT mice (Figure 4-4D and E). The concentration of PhA detectable in the WT mice after oral gavage in this study were at the lower limit of quantification (LLOQ), whereas in Bcrp KO mice serum, the concentration of $\mathrm{PhA}$ was significantly higher than LLOQ. This data is in conformation with our previous observation in rats, where $\mathrm{PhA}$ could only be detected in the Bcrp-Pgp dKO and not in the WT rat plasma, and also reports by Jonker et al. that Bcrp KO mice are 100-fold more sensitive to developing phototoxicity from $\mathrm{PhA}$ [92]. The difference in fold increase between whole body fluorescence (3.5fold) and serum concentration (50 fold) could be due to a higher background fluorescence in the whole-body fluorescence imaging, especially from unabsorbed $\mathrm{PhA}$ remaining in the gut, compared to the very low concentration of PhA in serum of WT mice. Thus, serum fluorescence of $\mathrm{PhA}$ might be a more useful tool than whole body fluorescence for elucidating the DDI of weak inhibitors of Bcrp. On the other hand, the advantages of 
using whole body fluorescence of the Bcrp substrate $\mathrm{PhA}$ to screen for oral DDI come from: (a) the ease and noninvasive nature of its detection, (b) that animals do not need to be sacrificed can be used repeatedly, (c) the ability to longitudinally monitor changes in Bcrp substrate tissue distribution into other organs in the presence of systemic inhibition of Bcrp, and (d) the possibility of transforming it into a better high-throughput method.

The dynamic range of $\mathrm{PhA}$ fluorescence, both whole body, and in serum, of WT and Bcrp KO mice, allowed us to rapidly examine the effect of Bcrp inhibitors on the whole-body fluorescence as well as serum concentration of $\mathrm{PhA}$. For an orally administered Bcrp inhibitor drug, if [(dose $/ 250 \mathrm{ml}) /$ IC50 for Bcrp] $\geq 10$, or Igut $/ \mathrm{IC} 50 \geq$ 10, FDA recommends analysis of possible oral Bcrp inhibition-mediated DDI [1]. Hence, we specifically chose drugs that would be predicted to cause a Bcrp-mediated DDI in vivo. For pantoprazole- (PPZ), elacridar- (ECD), curcumin- (CCM) and lapatinib (LPB)mediated clinical DDI liability, Igut/IC50 was obtained from literature reports, and are significantly higher than 10 [71]. For sorafenib (SFB), Igut/IC50 when calculated from maximum reported dose of $400 \mathrm{mg}$ BID data [305] and was found to be $>10$ (Table 4-1). Clinical DDI liability of dasatinib with Bcrp substrates was not reported, and hence was selected as a negative control in the experiment.

Based on reported pharmacokinetic (PK) parameters in mice, and associated clinically relevant doses and observed IC50 values, we also calculated the Igut/IC50 in mice assuming the gut volume in mouse to be $0.8 \mathrm{~mL}$ [306]. For the selected inhibitors Igut/IC50 values in mice are also $\geq 10$ and thus, according to FDA criteria, are expected to show preclinical DDI in mice (Table 4-1). Since, only the soluble fraction of the drug would be available at the enterocytes to inhibit Bcrp, we also calculated the gut soluble

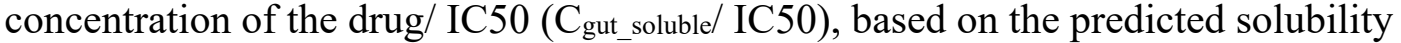
values obtained from DrugBank database (https://www.drugbank.ca). Considering these

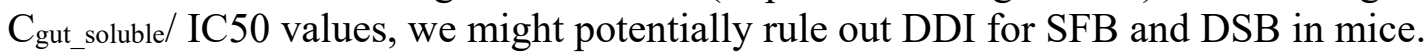
Cgut_soluble/ IC50 for CCM was also < 10. Kusuhara et al. dosed $300 \mathrm{mg} / \mathrm{kg} \mathrm{CCM} 1 \mathrm{hr}$ prior to sulfasalazine oral dosing in mice and observed an 8 -fold increase in sulfasalazine plasma AUC [259]. We have used the same curcumin dose and thus we expected CCMmediated inhibition of gut Bcrp would increase PhA serum AUC.

FDA also indicated that assessment of DDI is not required if there is no safety concern for the Bcrp substrate distribution in tissues [1]. Therefore, apart from inhibition of Bcrp at the gut, consideration is also required for systemic inhibition of Bcrp. To assess systemic Bcrp inhibition, we considered the reported $\mathrm{C}_{\max } / \mathrm{IC} 50$ for the Bcrp inhibitor drugs [71]. Systemic inhibition for SFB, ECD and LPB were expected based on the published $\mathrm{C}_{\max }$ in mice (Table 4-1) and observed IC50 values. But, for SFB, reported $\mathrm{C}_{\max }$ is associated with an enabling formulation [166], compared to a suspension formulation used in our study. Therefore, we would expect a lower $\mathrm{C}_{\max }$ and thus lack of systemic DDI in mice. Of note, based on clinically obtained $\mathrm{C}_{\max }$ of sorafenib [305], $\mathrm{C}_{\max } / \mathrm{IC} 50$ is 2 , suggesting that a systemic Bcrp inhibition by sorafenib may be expected. However, for a highly plasma protein bound drug such as sorafenib, unbound fraction in plasma $(\mathrm{Fu})$ is $0.3 \%$ [307]; unbound concentration in plasma may not be sufficient to exhibit a systemic inhibition of Bcrp. 
Physiochemical properties such as $\log \mathrm{P}$ and solubility values for the selected drugs were obtained from DrugBank database (https://www.drugbank.ca) to understand the possible disposition characteristic and gut solubility. Among the selected inhibitors, LPB, SFB and ECD have high logP or lipid permeability, thus probably have higher tissue distribution compared to the other inhibitors. Together with their low IC50 value for Bcrp, ECD and LPB might show systemic inhibition of Bcrp. On the other hand, PPZ has the highest predicted solubility, thus probably having fast absorption as well as faster transit than other selected inhibitors.

Our results with whole body fluorescence imaging (Figure 4-6 and Figure 4-7A-C) show increased whole-body fluorescence of $\mathrm{PhA}$ in mice administered the Bcrp inhibitors LPB, ECD and CCM. The AUCFL 0-6h for LPB, CCM and ECD were 2.4, 2.3 and 2.2-fold significantly higher than the control mice. A similar trend was observed for the serum concentration data (Figure 4-7D-F), with LPB having the highest fold change (13.8 fold) increase compared to controls, followed by CCM (7.8 fold), ECD (5.17 fold), PPZ (2.02 fold) and SFB (1.45 fold). Taken together, the serum concentration and imaging data provide evidence that PhA can be used for identification of oral DDIs associated with Bcrp. The only exception between these two methods was the observed lower AUC FL 0-6h for PPZ. Since PPZ solubility is the highest among all selected inhibitors, it is possible that it has a faster GI transit compared to the other drugs, leading to short-lasting inhibition of Bcrp at the upper gastro intestinal tract (GIT). Another possibility is the effect of altered $\mathrm{pH}$ on $\mathrm{PhA}$ fluorescence in presence of proton pump inhibitor PPZ. When mice were administered CCM with PhA, we observed a 7.79-fold increase in serum fluorescence of $\mathrm{PhA}$, while Kusuhara et al. observed an 8-fold increase in sulfasalazine plasma exposure in presence of CCM at a dose of 300 to $400 \mathrm{mg} / \mathrm{kg}$ [259], further corroborating that PhA might be utilized as an in vivo probe for Bcrp function. FDA Drug Interactions \& Labeling guideline also mentions that $\mathrm{a} \geq 1.5$-fold increase in sulfasalazine AUC when concomitantly dosed with a Bcrp inhibitor should be considered an in vivo inhibition of Bcrp. According to these criteria, PPZ, ECD, CCM and LPB showed in vivo inhibition of Bcrp-mediated PhA transport in our studies.

Measurement of serum concentration of $\mathrm{PhA}$ by fluorescence spectrometry-based methods has some advantages of creating a faster and less expensive method for faster decision-making processes. However, we recognize that this method might be less specific than an HPLC-RF based method. When we used an HPLC-RF based detection of $\mathrm{PhA}$ for $100 \mathrm{mg} / \mathrm{kg}$ oral dose of PhA (data not shown) in WT, Bcrp KO and ECDpretreated mice, PhA could be detected at the maximum concentration only in the serum of Bcrp KO and ECD-treated WT mice, implying PhA as a source of serum fluorescence. For our screening studies, we used a $10 \mathrm{mg} / \mathrm{kg} \mathrm{PhA}$ dose; thus, the expected $\mathrm{PhA}$ concentrations would be below the detection limit of our analytical method. Therefore, we utilized a whole serum fluorescence spectrometry-based method and confirmed the detection of $\mathrm{PhA}$ by obtaining similar fluorescence spectra between $\mathrm{PhA}$ standard concentration, blank serum and inhibitor-treated serum (Figure 4-8) groups, implying absence of any other fluorescent molecule. 
In our experiments, the Bcrp inhibitors were administered to the mice $1 \mathrm{hr}$ prior to $\mathrm{PhA}$ dosing. A similar (1-hr prior) dosing schedule was also utilized to investigate CCMmediated DDI with sulfasalazine in mice [259]. However, according to the FDA guidelines, a concomitant dosing of the two drugs is suggested. Due to fast absorption of compounds like dasatinib, with a reported $\mathrm{T}_{\max }$ as early as 15 minutes in mice [308], it is possible that in our study we have underestimated the effect of Bcrp inhibition at the GIT. Therefore, further investigation and careful observation might be required for identification of DDIs of weaker Bcrp inhibitors such as dasatinib.

Further studies are also required to find out if $\mathrm{PhA}$ can be used as a probe for systemic Bcrp inhibition as well, such as with lapatinib known to exhibit systemic Bcrp inhibition. For utilization of this method to its fullest potential, a better understanding of $\mathrm{PhA}$ pharmacokinetic properties would also be required with more emphasis on its tissue distribution and clearance.

To our knowledge, this is the first report that shows a fluorescent Bcrp substrate $\mathrm{PhA}$ can be utilized for identification of in vivo DDI due to inhibition of Bcrp at the GIT and creates an opportunity for a high throughput in vivo Bcrp inhibitor assay. Also, utilization of serum fluorescence by simple spectrometry makes this method unique in that minimal technical resources are required for identification of Bcrp substrates. Furthermore, this method is in conformation with the 3R principal (replacement, reduction and refinement), as it would reduce the requirement of invasive studies thus significantly reducing the number of lab animals, at the same time creating high quality reliable data.

In conclusion, our study shows that dynamic fluorescence imaging in live animals utilizing $\mathrm{PhA}$ as a Bcrp probe can be used for identification of Bcrp inhibitors and to understand the DDI liability of such inhibitors. Further studies are required to determine if we can use this compound as a dietary marker of BCRP activity in the clinic, and that may be further utilized to understand systemic Bcrp inhibition. 


\section{CHAPTER 5. SUMMARY AND DISCUSSION}

\section{Summary}

The overall goal of this research work was to understand the function of BCRP and PGP efflux drug transporters in context of their endogenous, xenobiotic and dietary substrates. We found (1) a significant impact of Bcrp and Pgp on the endogenous metabolome encompassing dietary, gut microbiome as well as host-derived metabolites, (2) a distinct effect of these transporters on the pharmacokinetics and pharmacodynamics of the anesthetic/analgesic drug ketamine, and (3) that dietary Bcrp substrate Pheophorbide A may be utilized as an in vivo marker for possible identification of Bcrp inhibition-mediated drug-drug interactions.

We compared the CSF and plasma metabolomes as well as gene expression in kidney, liver and brain frontal cortex between wild-type (WT) and Bcrp-Pgp double knockout $(\mathrm{dKO})$ rats to understand the systemic effect of these transporters in rats (Chapter 2). Our results showed a significant difference in both CSF and plasma metabolomic signatures between the WT and dKO rats. The most significant feature in the plasma metabolome was altered fatty acid metabolites, which was further supported by the altered genes and metabolites associated with the peroxisome proliferator activity receptor (PPAR) pathway. We also observed changes in gut microbiota-derived metabolites in both CSF and plasma of dKO rats, indicating a possible change in the gut microbiome, as well as an effect of Bcrp and/or Pgp in regulating the absorption of microbiota-derived metabolites in blood, and also their permeability in CSF. Transcriptomic analysis further revealed alterations in pathways related to fatty acid metabolism, lipopolysaccharide response (again indicating an altered microbiome), circadian rhythm as well as oxidative stress response in the dKO rats, when compared to WT rats. Analysis of the altered CSF metabolites, utilizing an in silico approach and literature-based evidence, identified metabolites which may be putative Bcrp substrates. Overall our results identified a significant impact of Bcrp and Pgp drug transporters on the endogenous metabolome and biological pathways, without any dramatic compensatory changes in transporter or enzyme expression.

Apart from alteration of known Bcrp endogenous substrates, such as riboflavin and urate, we observed a significantly higher abundance of ketamine, an anesthetic/analgesic drug used in this study to anesthetize the rats, in the plasma and CSF of $\mathrm{dKO}$ rats. Thereafter, utilizing mice with genetic deletion of Bcrp, Pgp and both BcrpPgp, we showed that pharmacokinetics (PK) as well as pharmacodynamics (PD) of ketamine were affected by both Bcrp and Pgp transporters (Chapter 3). A recently published article also confirmed our finding that ketamine is a substrate of Pgp [98].

Our plasma metabolomics identified Pheophorbide $\mathrm{A}(\mathrm{PhA})$, a catabolite of chlorophyll found in regular rodent diet, only in the plasma of dKO rats, but not in WT rat plasma. Thereafter, we developed a PhA fluorescence-based in vivo assay using mice fed an alfalfa-free diet (devoid of PhA) and exogenously administered with $\mathrm{PhA}$, to 
identify possible oral drug-drug interactions (DDIs) associated with Bcrp inhibition (Chapter 4). We observed an increased PhA fluorescence, both whole-body and in serum, of Bcrp-knockout mice, and in mice pre-treated with known Bcrp inhibitors, compared to WT mice, administered with PhA, suggesting that our assay might identify possible oral DDIs due to inhibition of Bcrp at mouse enterocytes.

\section{Perspectives and Future Directions}

While drug transporters are important for transporting xenobiotic substrates, and thereby involved in therapeutic resistance, drug-drug or food-drug interactions, they are also important for transporting endogenous metabolite(s) and involved in many important physiological functions [14]. Therefore, a clear understanding of the endogenous function of transporters will help better elucidate the effect of loss of function mutation or pharmacological inhibition of these transporters, leading to identification of drugmetabolite-transporter interactions.

Xenobiotics are often substrates of multiple transporters and/or enzymes. Therefore, an altered transport function due to a secondary effect of absence or inhibition of other transporter(s) may lead to pharmacokinetic or pharmacodynamic changes of transporter substrates and/or DDIs. For example, Martinez et al. recently published that association of methotrexate clearance (cleared mainly by urinary excretion) and OATP1B1 polymorphism (a transporter of the liver) can be explained by communication of OATP1B1 transporter, via its endogenous metabolites, with OAT1/3 transporters involved in methotrexate excretion at the kidney [213].

Several uremic toxins that are substrates/inhibitors of OAT1/3, BCRP and MRP4, are also metabolites of the gut microbiota [234] [141] [126]. Several clinically used nonantibiotic drugs are also known to alter the gut microbiome [313]. Therefore, it can be hypothesized that loss-of-function mutation and/or pharmacological inhibition of transporter(s) can lead to remote signaling via the gut microbiome metabolites to alter the function of other transporter(s), leading to a change in not only metabolite homeostasis, but also altered pharmacokinetics of their xenobiotic substrates.

BCRP and PGP efflux transporters are highly expressed at the enterocytes in gastrointestinal tract, regulating the oral absorption of their substrates [11, 115, 177, 258, $259,270]$. Hence, while considering the effect of gut microbiota on systemic metabolites, a special consideration is needed for these efflux transporters that control the oral absorption of many different chemical class of compounds.

The transporters are also highly expressed at the blood brain barrier (BBB) and blood CSF barrier (BCSFB), regulating the brain or CSF permeability of their substrates $[169,171][144][46,155]$. Their effect on brain accumulation of BCRP and PGP substrates is so dramatic probably because of (i) lack of efficient metabolic clearance from brain, compared to liver that takes care of peripheral blood concentration and (ii) rate of clearance from brain is slower than from peripheral blood due to lower flow rate 
of CSF. Specifically, in adult humans, the total CSF volume turnover is about 4 times a day, with a CSF synthesis rate of $0.35-0.4 \mathrm{ml} / \mathrm{min}$ [314], compared to blood flow rate to brain which is about $700 \mathrm{ml} / \mathrm{min}$, and to liver which is $1.4 \mathrm{~L} / \mathrm{min}$ (http://rmipharmacokinetics.com/Physiological_parameters).

Therefore, the effect of dietary and microbiome metabolites, that are PGP and/or BCRP substrates/inhibitors, might be highly significant in brain as well. To this effect, our metabolomic analysis in rats identified abundance of many sulfate metabolites, possibly dietary or microbiome-generated, and the known Bcrp substrate urate in the CSF of dKO rats, compared to WT rats. Since Bcrp has high affinity for sulfated metabolites [127]; our results raise the possibility that BCRP might also be involved in controlling gut-brain chemical interactions. A recent finding showed that sulfated steroids, such as estradiol sulfate, in female mice urine, can activate vomeronasal sensory neurons (VSNs), which are part of accessory olfactory system (AOS), involved in social interaction in mice [315]. Therefore, BCRP, which is known to be a transporter of sulfated steroids [316], might have a more significant role to play at different tissues including brain, than being a mere xenobiotic transporter.

It is well known that the absence of Pgp in mice leads to an irritable bowel syndrome phenotype [51], and possible modification of the gut microbiome in absence of Pgp could be the leading cause for this phenotype [162]. Gut microbiome is also reported to change in gout patients, who have hyperuricemia [139]. Bcrp is involved in the intestinal secretion of uric acid, a decrease of which can lead to hyperuricemia [138] [114] and precipitation of gout in humans with BCRP polymorphism [24]. Therefore, alteration in the gut microbiome is possible in the absence or chronic inhibition of BCRP and/or PGP transporters, leading to an altered metabolome in the host. Gut microbiome has also been recently associated with disease conditions related to CNS [317] [318] [319]. While Bcrp and/or Pgp knockout mice do not show any obvious phenotype of CNS pathology, a behavioral understanding of rodent models of these diseases is not so common and more studies need to be done to understand the role of transporters in the CNS.

Recently published reports have highlighted the importance of understanding pharmacometabolomics, i.e., the effect of drug treatment on the metabolome, or a metabotype of drug action $[180,185]$. Lin et al. showed that pharmacometabolomic understanding can also help predict population variability in busulfan clearance [214]. Studies by Dr. Elizabeth de Lange's group at Leiden University further showed that integrated metabolomics and PK/PD studies can be greatly useful and provide significant insight into CNS drug discovery [320, 321]. Therefore, the concept of utilizing metabolomics for predicting drug pharmacokinetics and pharmacodynamics may not be a far-fetched hypothesis in near future. Knowledge integration of metabolomics of drug transporters and associated biomarker information, if possible, could be extremely helpful in understanding population variability in PK, drug response as well as toxicity associated with treatment and/or disease. 
To our knowledge, this is the first study reporting the metabolomic impact of Bcrp and Pgp drug transporters in rodents. In a preliminary experiment comparing the gut microbiome between WT and Bcrp KO mice by 16S ribosomal RNA sequencing of fecal samples, we have observed a significant difference in percentage abundance of gut microbial populations, as was suggested by our CSF and plasma metabolomic findings in rats (data not shown). Future studies should be directed towards metabolomic analysis of fecal, plasma and urine samples from these mice for integration with the microbiome abundance data leading to a better understanding of host-microbiome metabolomic interactions in absence of Bcrp transporter. Furthermore, metabolomic analyses of different models of gene deletion or inhibition of other transporters in a variety of matrices would be required for a better understanding of the Remote Sensing and Signaling concept [20].

We unexpectedly found an increase in ketamine abundance in plasma as well as CSF of Bcrp-Pgp dKO rats compared to WT rats and later confirmed that pharmacokinetics (PK) and pharmacodynamics (PD) of ketamine are affected by these transporters in mice [95]. Unlike prototypical Bcrp-Pgp substrates, ketamine whole brain homogenate concentration was found to be similar between WT, Bcrp or Pgp KO and $\mathrm{dKO}$ mice, whereas its anesthetic effect as measured by loss of righting reflex (dLORR) was found to be significantly higher in the $\mathrm{dKO}$ mice, followed by Pgp KO mice, compared to WT mice. Treatment with known Bcrp-Pgp inhibitor elacridar (ECD) also increased dLORR in the WT mice. Due to high passive permeability of ketamine, effect of efflux transporters in brain may not be similar to other substrates that show increased brain accumulation in absence of these transporters. The same was observed for another Pgp substrate terfenadine [97], another high permeability Pgp substrate, with no difference in brain uptake clearance between WT and Pgp KO mice. This raises the possibility of overlooking the effect of these transporters on certain compounds when only PK is considered as a discerning factor. In this regard, FDA guidance on assessing DDI states that the effect on distributing tissue(s)/organ(s) toxicity or efficacy needs to be considered when assessing DDIs. Therefore, we believe that the PD or toxicity of a drug, along with its PK, should be an additional factor of consideration during DDI assessment. In addition, long-term administration of ketamine is well-known to cause toxicity in the urinary bladder and kidney [252] [322] [323]. Therefore, based on our current knowledge of the effect of Pgp, Bcrp and OCT1/2 transporters on ketamine [98] [95], it will be interesting to see if these transporters play any role on the peripheral tissue toxicity of ketamine.

While our current investigation does not specifically identify any biomarker for Bcrp/Pgp activity, we identified several putative endogenous substrates of Bcrp and/or Pgp which might be interesting candidates for further investigation. However, our studies with $\mathrm{PhA}$ as a dietary marker of gastrointestinal Bcrp activity showed the possibility of utilizing a fluorescent substrate/marker for high-throughput screening of Bcrp inhibitors in vivo. A 50-fold window of difference in serum concentration of PhA between WT and Bcrp KO mice created a prospect for identification of Bcrp substrates in vivo by measuring serum fluorescence. Our results from rats on regular diet showed that $\mathrm{PhA}$ is detectable only in the plasma of Bcrp-Pgp dKO rats, but none in WT rats. This is 
interesting, as it creates an opportunity to utilize dietary $\mathrm{PhA}$, which is a chlorophyll catabolite, to be utilized as a dietary biomarker of BCRP activity in humans as well, since chlorophyll is a dietary component in humans. A future modification of this study with more soluble PhA analogues that are also Bcrp substrates and gives a better tissue distribution and absorption compared to $\mathrm{PhA}$ can also be useful to assess systemic inhibition of Bcrp and associated increase in tissue concentration of its substrates.

In conclusion, this research work created a new understanding of the endogenous role of Bcrp and Pgp efflux transporters, their effect on the PK and PD of the anesthetic/analgesic drug ketamine, and development of an assay for identification of Bcrp inhibitors in mice. Further studies need to be done with focused goals specifically (i) to elucidate the effect of the transporters on microbiome and associated metabolic changes, (ii) how peripheral toxicity can be modified by efflux transporters Bcrp and Pgp, with a model substrate ketamine, (iii) modification of the existing protocol of $\mathrm{PhA}$ in vivo assay, with carefully selected Bcrp substrate(s) and inhibitors for developing a robust fluorescence-based assay that can detect systemic as well as oral Bcrp inhibition in vivo, and (iv) elucidating the pharmacometabolomics of drugs that interact with Bcrp and Pgp transporters, thereby creating better understanding of long term systemic inhibition of these transporters. 


\section{LIST OF REFERENCES}

1. USFDA-CDER, Guidance for Industry: In vitro Metabolism and Transporter Mediated Drug-Drug Interaction Studies. FDA, Maryland, 2017.

2. EMA, Guideline on the investigation of drug interactions. 2012.

3. Glaeser, H., Importance of P-glycoprotein for Drug-Drug Interactions, in Drug Transporters, M.F. Fromm and R.B. Kim, Editors. 2011, Springer Berlin Heidelberg: Berlin, Heidelberg. p. 285-297.

4. Smith, N.F., W.D. Figg, and A. Sparreboom, Role of the liver-specific transporters OATP1B1 and OATP1B3 in governing drug elimination. Expert Opin Drug Metab Toxicol, 2005. 1(3): p. 429-45.

5. $\quad$ Nies, A.T., et al., Organic Cation Transporters (OCTs, MATEs), In vitro and In vivo Evidence for the Importance in Drug Therapy, in Drug Transporters, M.F. Fromm and R.B. Kim, Editors. 2011, Springer Berlin Heidelberg: Berlin, Heidelberg. p. 105-167.

6. Schwabedissen, H.E.M.z. and H.K. Kroemer, In vitro and In vivo Evidence for the Importance of Breast Cancer Resistance Protein Transporters (BCRP/MXR/ABCP/ABCG2), in Drug Transporters, M.F. Fromm and R.B. Kim, Editors. 2011, Springer Berlin Heidelberg: Berlin, Heidelberg. p. 325-371.

7. Burckhardt, G. and B.C. Burckhardt, In vitro and In vivo Evidence of the Importance of Organic Anion Transporters (OATs) in Drug Therapy, in Drug Transporters, M.F. Fromm and R.B. Kim, Editors. 2011, Springer Berlin Heidelberg: Berlin, Heidelberg. p. 29-104.

8. Tamai, I., Pharmacological and pathophysiological roles of carnitine/organic cation transporters (OCTNs: SLC22A4, SLC22A5 and Slc22a21). Biopharm Drug Dispos, 2013. 34(1): p. 29-44.

9. van Herwaarden, A.E., et al., Multidrug transporter ABCG2/breast cancer resistance protein secretes riboflavin (vitamin B2) into milk. Mol Cell Biol, 2007. 27(4): p. 1247-53.

10. Barile, M., et al., Riboflavin transport and metabolism in humans. J Inherit Metab Dis, 2016. 39(4): p. 545-57.

11. Bruhn, O. and I. Cascorbi, Polymorphisms of the drug transporters ABCB1, $\mathrm{ABCG} 2, \mathrm{ABCC} 2$ and $\mathrm{ABCC} 3$ and their impact on drug bioavailability and clinical relevance. Expert Opin Drug Metab Toxicol, 2014. 10(10): p. 1337-54. 
12. Sissung, T.M., et al., Transporter pharmacogenetics: transporter polymorphisms affect normal physiology, diseases, and pharmacotherapy. Discov Med, 2012. 13(68): p. 19-34.

13. Yee, S.W., et al., Influence of Transporter Polymorphisms on Drug Disposition and Response: A Perspective from the International Transporter Consortium. Clin Pharmacol Ther, 2018.

14. Alexander, S.P., et al., THE CONCISE GUIDE TO PHARMACOLOGY 2017/18: Transporters. Br J Pharmacol, 2017. 174 Suppl 1: p. S360-S446.

15. Andersen, V., et al., Novel understanding of $A B C$ transporters $A B C B 1 / M D R / P-$ glycoprotein, $\mathrm{ABCC} / \mathrm{MRP} 2$, and $\mathrm{ABCG} 2 / \mathrm{BCRP}$ in colorectal pathophysiology. World J Gastroenterol, 2015. 21(41): p. 11862-76.

16. Brant, S.R., et al., MDR1 Ala893 polymorphism is associated with inflammatory bowel disease. Am J Hum Genet, 2003. 73(6): p. 1282-92.

17. Rees, D.C., E. Johnson, and O. Lewinson, ABC transporters: the power to change. Nat Rev Mol Cell Biol, 2009. 10(3): p. 218-27.

18. Vasiliou, V., K. Vasiliou, and D.W. Nebert, Human ATP-binding cassette (ABC) transporter family. Hum Genomics, 2009. 3(3): p. 281-90.

19. Hediger, M.A., et al., The ABCs of membrane transporters in health and disease (SLC series): introduction. Mol Aspects Med, 2013. 34(2-3): p. 95-107.

20. Nigam, S.K., et al., The organic anion transporter (OAT) family: a systems biology perspective. Physiol Rev, 2015. 95(1): p. 83-123.

21. Zhao, M., et al., Meta-analysis of the interaction between serotonin transporter promoter variant, stress, and posttraumatic stress disorder. Sci Rep, 2017. 7(1): p. 16532.

22. Leung, E., et al., Polymorphisms in the organic cation transporter genes SLC22A4 and SLC22A5 and Crohn's disease in a New Zealand Caucasian cohort. Immunol Cell Biol, 2006. 84(2): p. 233-6.

23. Marques, F.Z., et al., A polymorphism in the norepinephrine transporter gene is associated with affective and cardiovascular disease through a microRNA mechanism. Mol Psychiatry, 2017. 22(1): p. 134-141.

24. Dehghan, A., et al., Association of three genetic loci with uric acid concentration and risk of gout: a genome-wide association study. Lancet, 2008. 372(9654): p. 1953-61. 
25. Giacomini, K.M., et al., Membrane transporters in drug development. Nat Rev Drug Discov, 2010. 9(3): p. 215-36.

26. Matherly, L.H., M.R. Wilson, and Z. Hou, The major facilitative folate transporters solute carrier 19A1 and solute carrier 46A1: biology and role in antifolate chemotherapy of cancer. Drug Metab Dispos, 2014. 42(4): p. 632-49.

27. Roth, M., A. Obaidat, and B. Hagenbuch, OATPs, OATs and OCTs: the organic anion and cation transporters of the SLCO and SLC22A gene superfamilies. Br J Pharmacol, 2012. 165(5): p. 1260-87.

28. König, J., Uptake Transporters of the Human OATP Family, in Drug Transporters, M.F. Fromm and R.B. Kim, Editors. 2011, Springer Berlin Heidelberg: Berlin, Heidelberg. p. 1-28.

29. Obaidat, A., M. Roth, and B. Hagenbuch, The expression and function of organic anion transporting polypeptides in normal tissues and in cancer. Annu Rev Pharmacol Toxicol, 2012. 52: p. 135-51.

30. Karlgren, M., et al., Classification of inhibitors of hepatic organic anion transporting polypeptides (OATPs): influence of protein expression on drug-drug interactions. J Med Chem, 2012. 55(10): p. 4740-63.

31. Tweedie, D., et al., Transporter studies in drug development: experience to date and follow-up on decision trees from the International Transporter Consortium. Clin Pharmacol Ther, 2013. 94(1): p. 113-25.

32. Shafiu, M., et al., Urate transporter gene SLC22A12 polymorphisms associated with obesity and metabolic syndrome in Caucasians with hypertension. Kidney Blood Press Res, 2012. 35(6): p. 477-82.

33. So, A. and B. Thorens, Uric acid transport and disease. J Clin Invest, 2010. 120(6): p. 1791-9.

34. Dean, M., A. Rzhetsky, and R. Allikmets, The human ATP-binding cassette (ABC) transporter superfamily. Genome Res, 2001. 11(7): p. 1156-66.

35. Theodoulou, F.L. and I.D. Kerr, ABC transporter research: going strong 40 years on. Biochem Soc Trans, 2015. 43(5): p. 1033-40.

36. Spengler, G., et al., New Roads Leading to Old Destinations: Efflux Pumps as Targets to Reverse Multidrug Resistance in Bacteria. Molecules, 2017. 22(3).

37. Velamakanni, S., et al., ABCG transporters: structure, substrate specificities and physiological roles : a brief overview. J Bioenerg Biomembr, 2007. 39(5-6): $\mathrm{p}$. 465-71. 
38. Schinkel, A.H. and J.W. Jonker, Mammalian drug efflux transporters of the ATP binding cassette (ABC) family: an overview. Adv Drug Deliv Rev, 2003. 55(1): p. 3-29.

39. Quazi, F. and R.S. Molday, Lipid transport by mammalian ABC proteins. Essays Biochem, 2011. 50(1): p. 265-90.

40. Jiang, L., et al., Polymorphism analysis of the ABCA3 gene: association with neonatal respiratory distress syndrome in preterm infants. Chin Med J (Engl), 2012. 125(9): p. 1594-8.

41. Schulz, H.L., et al., Mutation Spectrum of the ABCA4 Gene in 335 Stargardt Disease Patients From a Multicenter German Cohort-Impact of Selected Deep Intronic Variants and Common SNPs. Invest Ophthalmol Vis Sci, 2017. 58(1): p. 394-403.

42. Hollingworth, P., et al., Common variants at ABCA7, MS4A6A/MS4A4E, EPHA1, CD33 and CD2AP are associated with Alzheimer's disease. Nat Genet, 2011. 43(5): p. 429-35.

43. Droge, C., et al., Sequencing of FIC1, BSEP and MDR3 in a large cohort of patients with cholestasis revealed a high number of different genetic variants. J Hepatol, 2017. 67(6): p. 1253-1264.

44. Khor, C.C., et al., Genome-wide linkage and association mapping identify susceptibility alleles in ABCC4 for Kawasaki disease. J Med Genet, 2011. 48(7): p. 467-72.

45. Bai, Y., M. Li, and T.C. Hwang, Structural basis for the channel function of a degraded ABC transporter, CFTR (ABCC7). J Gen Physiol, 2011. 138(5): p. 495507.

46. Borst, P. and A.H. Schinkel, P-glycoprotein ABCB1: a major player in drug handling by mammals. J Clin Invest, 2013. 123(10): p. 4131-3.

47. Fukuda, Y. and J.D. Schuetz, ABC transporters and their role in nucleoside and nucleotide drug resistance. Biochem Pharmacol, 2012. 83(8): p. 1073-83.

48. Schinkel, A.H., et al., Disruption of the mouse mdrla P-glycoprotein gene leads to a deficiency in the blood-brain barrier and to increased sensitivity to drugs. Cell, 1994. 77(4): p. 491-502.

49. Schinkel, A.H., et al., P-glycoprotein in the blood-brain barrier of mice influences the brain penetration and pharmacological activity of many drugs. J Clin Invest, 1996. 97(11): p. 2517-24. 
50. Schinkel, A.H., et al., Absence of the mdrla P-Glycoprotein in mice affects tissue distribution and pharmacokinetics of dexamethasone, digoxin, and cyclosporin A. J Clin Invest, 1995. 96(4): p. 1698-705.

51. Panwala, C.M., J.C. Jones, and J.L. Viney, A novel model of inflammatory bowel disease: mice deficient for the multiple drug resistance gene, mdr1a, spontaneously develop colitis. J Immunol, 1998. 161(10): p. 5733-44.

52. Fujinami, K., et al., Clinical and molecular characteristics of childhood-onset Stargardt disease. Ophthalmology, 2015. 122(2): p. 326-34.

53. Kerr, I.D., A.J. Haider, and I.C. Gelissen, The ABCG family of membraneassociated transporters: you don't have to be big to be mighty. Br J Pharmacol, 2011. 164(7): p. 1767-79.

54. Nakayama, A., et al., ABCG2 is a high-capacity urate transporter and its genetic impairment increases serum uric acid levels in humans. Nucleosides Nucleotides Nucleic Acids, 2011. 30(12): p. 1091-7.

55. Allen, J.D., et al., The mouse Bcrp1/Mxr/Abcp gene: amplification and overexpression in cell lines selected for resistance to topotecan, mitoxantrone, or doxorubicin. Cancer Res, 1999. 59(17): p. 4237-41.

56. Smit, J.W., et al., Absence or pharmacological blocking of placental Pglycoprotein profoundly increases fetal drug exposure. J Clin Invest, 1999. 104(10): p. 1441-7.

57. Yates, C.R., et al., Structural determinants of P-glycoprotein-mediated transport of glucocorticoids. Pharm Res, 2003. 20(11): p. 1794-803.

58. Oude Elferink, R.P. and J. Zadina, MDR1 P-glycoprotein transports endogenous opioid peptides. Peptides, 2001. 22(12): p. 2015-20.

59. Keppler, D., Multidrug Resistance Proteins (MRPs, ABCCs): Importance for Pathophysiology and Drug Therapy, in Drug Transporters, M.F. Fromm and R.B. Kim, Editors. 2011, Springer Berlin Heidelberg: Berlin, Heidelberg. p. 299-323.

60. Kochel, T.J., et al., Multiple drug resistance-associated protein (MRP4) exports prostaglandin E2 (PGE2) and contributes to metastasis in basal/triple negative breast cancer. Oncotarget, 2017. 8(4): p. 6540-6554.

61. Kool, M., et al., Analysis of expression of cMOAT (MRP2), MRP3, MRP4, and MRP5, homologues of the multidrug resistance-associated protein gene (MRP1), in human cancer cell lines. Cancer Res, 1997. 57(16): p. 3537-47. 
62. Doyle, L.A., et al., A multidrug resistance transporter from human MCF-7 breast cancer cells. Proc Natl Acad Sci U S A, 1998. 95(26): p. 15665-70.

63. Shen, D.W., et al., Human multidrug-resistant cell lines: increased mdr1 expression can precede gene amplification. Science, 1986. 232(4750): p. 643-5.

64. Chang, C., et al., Pharmacophore-based discovery of ligands for drug transporters. Adv Drug Deliv Rev, 2006. 58(12-13): p. 1431-50.

65. Chang, C., et al., Rapid identification of P-glycoprotein substrates and inhibitors. Drug Metab Dispos, 2006. 34(12): p. 1976-84.

66. Ekins, S., et al., Three-dimensional quantitative structure-activity relationships of inhibitors of P-glycoprotein. Mol Pharmacol, 2002. 61(5): p. 964-73.

67. Matsson, P., et al., A global drug inhibition pattern for the human ATP-binding cassette transporter breast cancer resistance protein (ABCG2). J Pharmacol Exp Ther, 2007. 323(1): p. 19-30.

68. Pan, Y., P.P. Chothe, and P.W. Swaan, Identification of novel breast cancer resistance protein (BCRP) inhibitors by virtual screening. Mol Pharm, 2013. 10(4): p. 1236-48.

69. Szafraniec, M.J., et al., Determinants of the activity and substrate recognition of breast cancer resistance protein (ABCG2). Drug Metab Rev, 2014. 46(4): p. 45974.

70. Cascorbi, I., P-glycoprotein: Tissue Distribution, Substrates, and Functional Consequences of Genetic Variations, in Drug Transporters, M.F. Fromm and R.B. Kim, Editors. 2011, Springer Berlin Heidelberg: Berlin, Heidelberg. p. 261-283.

71. Lee, C.A., et al., Breast cancer resistance protein (ABCG2) in clinical pharmacokinetics and drug interactions: practical recommendations for clinical victim and perpetrator drug-drug interaction study design. Drug Metab Dispos, 2015. 43(4): p. 490-509.

72. Wooten, J.M., A Brief Drug Class Review: Considerations for Statin Use, Toxicity, and Drug Interactions. South Med J, 2018. 111(1): p. 39-44.

73. Pitre, A., et al., An unexpected protein interaction promotes drug resistance in leukemia. Nat Commun, 2017. 8(1): p. 1547.

74. Crawford, R.R., et al., Beyond Competitive Inhibition: Regulation of ABC Transporters by Kinases and Protein-Protein Interactions as Potential Mechanisms of Drug-Drug Interactions. Drug Metab Dispos, 2018. 46(5): p. 567-580. 
75. Sprowl, J.A., et al., A phosphotyrosine switch regulates organic cation transporters. Nat Commun, 2016. 7: p. 10880.

76. Johnson, E.J., et al., Prioritizing pharmacokinetic drug interaction precipitants in natural products: application to OATP inhibitors in grapefruit juice. Biopharm Drug Dispos, 2017. 38(3): p. 251-259.

77. Bhardwaj, R.K., et al., Piperine, a major constituent of black pepper, inhibits human P-glycoprotein and CYP3A4. J Pharmacol Exp Ther, 2002. 302(2): p. 64550 .

78. Marchetti, S., et al., Concise review: Clinical relevance of drug drug and herb drug interactions mediated by the ABC transporter ABCB1 (MDR1, Pglycoprotein). Oncologist, 2007. 12(8): p. 927-41.

79. Peltoniemi, M.A., et al., St John's wort greatly decreases the plasma concentrations of oral S-ketamine. Fundam Clin Pharmacol, 2012. 26(6): p. 74350 .

80. USFDA-CDER, Guidance for Industry: Clinical Drug Interaction Studies Study Design, Data Analysis, and Clinical Implications Guidance for Industry. FDA, Maryland, 2017.

81. Yasuda, K., et al., Interaction of cytochrome P450 3A inhibitors with Pglycoprotein. J Pharmacol Exp Ther, 2002. 303(1): p. 323-32.

82. Drozdzik, M. and S. Oswald, Expression and Regulation of Drug Transporters and Metabolizing Enzymes in the Human Gastrointestinal Tract. Curr Med Chem, 2016. 23(39): p. 4468-4489.

83. Lim, H.Y., Q.S. Ho, and K.P. Wong, Interplay of metabolizing enzymes and transporter of xenobiotics. Xenobiotica, 2016. 46(1): p. 25-33.

84. Shi, S. and Y. Li, Interplay of Drug-Metabolizing Enzymes and Transporters in Drug Absorption and Disposition. Curr Drug Metab, 2014. 15(10): p. 915-41.

85. Neuvonen, P.J., Drug interactions with HMG-CoA reductase inhibitors (statins): the importance of CYP enzymes, transporters and pharmacogenetics. Curr Opin Investig Drugs, 2010. 11(3): p. 323-32.

86. Englund, G., et al., Cytochrome p450 inhibitory properties of common efflux transporter inhibitors. Drug Metab Dispos, 2014. 42(3): p. 441-7.

87. Oostendorp, R.L., et al., Organic anion-transporting polypeptide 1B1 mediates transport of Gimatecan and BNP1350 and can be inhibited by several classic 
ATP-binding cassette $(\mathrm{ABC}) \mathrm{B} 1$ and/or ABCG2 inhibitors. Drug Metab Dispos, 2009. 37(4): p. 917-23.

88. Brouwer, K.L., et al., In vitro methods to support transporter evaluation in drug discovery and development. Clin Pharmacol Ther, 2013. 94(1): p. 95-112.

89. Zamek-Gliszczynski, M.J., et al., ITC recommendations for transporter kinetic parameter estimation and translational modeling of transport-mediated PK and DDIs in humans. Clin Pharmacol Ther, 2013. 94(1): p. 64-79.

90. Brouwer, K.L., et al., In vitro methods to support transporter evaluation in drug discovery and development. Clin Pharmacol Ther, 2013. 94(1): p. 95-112.

91. Zamek-Gliszczynski, M.J., et al., Minor compensatory changes in SAGE Mdr1a (P-gp), Bcrp, and Mrp2 knockout rats do not detract from their utility in the study of transporter-mediated pharmacokinetics. Drug Metab Dispos, 2013. 41(6): p. $1174-8$.

92. Jonker, J.W., et al., The breast cancer resistance protein protects against a major chlorophyll-derived dietary phototoxin and protoporphyria. Proc Natl Acad Sci U S A, 2002. 99(24): p. 15649-54.

93. Kodaira, H., et al., Quantitative Investigation of the Brain-to-cerebrospinal Fluid Unbound Drug Concentration Ratio Under Steady-state Conditions in Rats Using a Pharmacokinetic Model and Scaling Factors for Active Efflux Transporters. Drug Metab Dispos, 2014.

94. Lagas, J.S., et al., Breast cancer resistance protein and P-glycoprotein limit sorafenib brain accumulation. Mol Cancer Ther, 2010. 9(2): p. 319-26.

95. Ganguly, S., et al., Ketamine pharmacokinetics and pharmacodynamics are altered by Pgp and Bcrp efflux transporters in mice. Drug Metab Dispos, 2018.

96. Mahar Doan, K.M., et al., Steady-state brain concentrations of antihistamines in rats: interplay of membrane permeability, P-glycoprotein efflux and plasma protein binding. Pharmacology, 2004. 72(2): p. 92-8.

97. Zhao, R., et al., Fexofenadine brain exposure and the influence of blood-brain barrier P-glycoprotein after fexofenadine and terfenadine administration. Drug Metab Dispos, 2009. 37(3): p. 529-35.

98. Keiser, M., M. Hasan, and S. Oswald, Affinity of Ketamine to Clinically Relevant Transporters. Mol Pharm, 2018. 15(1): p. 326-331.

99. Mairinger, S., et al., PET and SPECT radiotracers to assess function and expression of $\mathrm{ABC}$ transporters in vivo. Curr Drug Metab, 2011. 12(8): p. 774-92. 
100. Milak, M.S., et al., Higher 5-HT1A autoreceptor binding as an endophenotype for major depressive disorder identified in high risk offspring - A pilot study. Psychiatry Res Neuroimaging, 2018. 276: p. 15-23.

101. Robey, R.W., et al., ABCG2: a perspective. Adv Drug Deliv Rev, 2009. 61(1): p. 3-13.

102. Stacy, A.E., P.J. Jansson, and D.R. Richardson, Molecular pharmacology of ABCG2 and its role in chemoresistance. Mol Pharmacol, 2013. 84(5): p. 655-69.

103. Maliepaard, M., et al., Subcellular localization and distribution of the breast cancer resistance protein transporter in normal human tissues. Cancer Res, 2001. 61(8): p. 3458-64.

104. Vlaming, M.L., J.S. Lagas, and A.H. Schinkel, Physiological and pharmacological roles of ABCG2 (BCRP): recent findings in Abcg2 knockout mice. Adv Drug Deliv Rev, 2009. 61(1): p. 14-25.

105. Gutmann, H., et al., Distribution of breast cancer resistance protein (BCRP/ABCG2) mRNA expression along the human GI tract. Biochem Pharmacol, 2005. 70(5): p. 695-9.

106. Zhou, S., et al., Bcrp1 gene expression is required for normal numbers of side population stem cells in mice, and confers relative protection to mitoxantrone in hematopoietic cells in vivo. Proc Natl Acad Sci U S A, 2002. 99(19): p. 12339-44.

107. Zhou, S., et al., The ABC transporter Bcrp1/ABCG2 is expressed in a wide variety of stem cells and is a molecular determinant of the side-population phenotype. Nat Med, 2001. 7(9): p. 1028-34.

108. Miranda-Lorenzo, I., et al., Intracellular autofluorescence: a biomarker for epithelial cancer stem cells. Nat Methods, 2014. 11(11): p. 1161-9.

109. Krishnamurthy, P., et al., The stem cell marker Bcrp/ABCG2 enhances hypoxic cell survival through interactions with heme. J Biol Chem, 2004. 279(23): p. 24218-25.

110. Meissner, K., et al., The ATP-binding cassette transporter ABCG2 (BCRP), a marker for side population stem cells, is expressed in human heart. J Histochem Cytochem, 2006. 54(2): p. 215-21.

111. Zamber, C.P., et al., Natural allelic variants of breast cancer resistance protein (BCRP) and their relationship to BCRP expression in human intestine. Pharmacogenetics, 2003. 13(1): p. 19-28. 
112. Ieiri, I., Functional significance of genetic polymorphisms in P-glycoprotein (MDR1, ABCB1) and breast cancer resistance protein (BCRP, ABCG2). Drug Metab Pharmacokinet, 2012. 27(1): p. 85-105.

113. Sakiyama, M., et al., Ethnic differences in ATP-binding cassette transporter, subfamily G, member 2 (ABCG2/BCRP): genotype combinations and estimated functions. Drug Metab Pharmacokinet, 2014. 29(6): p. 490-2.

114. Ichida, K., et al., Decreased extra-renal urate excretion is a common cause of hyperuricemia. Nat Commun, 2012. 3: p. 764.

115. Sparreboom, A., et al., Effect of ABCG2 genotype on the oral bioavailability of topotecan. Cancer Biol Ther, 2005. 4(6): p. 650-8.

116. Matsuo, H., et al., ABCG2 variant has opposing effects on onset ages of Parkinson's disease and gout. Ann Clin Transl Neurol, 2015. 2(3): p. 302-6.

117. Li, W., et al., ABCB1 3435TT and ABCG2 421CC genotypes were significantly associated with longer progression-free survival in Chinese breast cancer patients. Oncotarget, 2017. 8(67): p. 111041-111052.

118. Schumacher, T. and R.A. Benndorf, ABC Transport Proteins in Cardiovascular Disease-A Brief Summary. Molecules, 2017. 22(4).

119. Zelinski, T., et al., ABCG2 null alleles define the $\operatorname{Jr}(\mathrm{a}-)$ blood group phenotype. Nat Genet, 2012. 44(2): p. 131-2.

120. Saison, C., et al., Null alleles of ABCG2 encoding the breast cancer resistance protein define the new blood group system Junior. Nat Genet, 2012. 44(2): p. 1747.

121. Mao, Q. and J.D. Unadkat, Role of the breast cancer resistance protein (BCRP/ABCG2) in drug transport--an update. AAPS J, 2015. 17(1): p. 65-82.

122. Merino, G., et al., Breast cancer resistance protein (BCRP/ABCG2) transports fluoroquinolone antibiotics and affects their oral availability, pharmacokinetics, and milk secretion. Drug Metab Dispos, 2006. 34(4): p. 690-5.

123. Robey, R.W., et al., Mutations at amino-acid 482 in the ABCG2 gene affect substrate and antagonist specificity. Br J Cancer, 2003. 89(10): p. 1971-8.

124. Breedveld, P., et al., The effect of low $\mathrm{pH}$ on breast cancer resistance protein (ABCG2)-mediated transport of methotrexate, 7-hydroxymethotrexate, methotrexate diglutamate, folic acid, mitoxantrone, topotecan, and resveratrol in in vitro drug transport models. Mol Pharmacol, 2007. 71(1): p. 240-9. 
125. Desuzinges-Mandon, E., et al., ABCG2 transports and transfers heme to albumin through its large extracellular loop. J Biol Chem, 2010. 285(43): p. 33123-33.

126. Mutsaers, H.A., et al., Uremic toxins inhibit transport by breast cancer resistance protein and multidrug resistance protein 4 at clinically relevant concentrations. PLoS One, 2011. 6(4): p. e18438.

127. van de Wetering, K. and S. Sapthu, ABCG2 functions as a general phytoestrogen sulfate transporter in vivo. FASEB J, 2012. 26(10): p. 4014-24.

128. Gao, C., et al., Hepatic Transport of 25-Hydroxyvitamin D3 Conjugates: A Mechanism of 25-Hydroxyvitamin D3 Delivery to the Intestinal Tract. Drug Metab Dispos, 2018. 46(5): p. 581-591.

129. Krishnamurthy, P. and J.D. Schuetz, Role of ABCG2/BCRP in biology and medicine. Annu Rev Pharmacol Toxicol, 2006. 46: p. 381-410.

130. Agarwal, S., et al., Distribution of gefitinib to the brain is limited by Pglycoprotein $(\mathrm{ABCB} 1)$ and breast cancer resistance protein (ABCG2)-mediated active efflux. J Pharmacol Exp Ther, 2010. 334(1): p. 147-55.

131. de Vries, N.A., et al., P-glycoprotein and breast cancer resistance protein: two dominant transporters working together in limiting the brain penetration of topotecan. Clin Cancer Res, 2007. 13(21): p. 6440-9.

132. Shen, J., et al., Compartment-specific roles of ATP-binding cassette transporters define differential topotecan distribution in brain parenchyma and cerebrospinal fluid. Cancer Res, 2009. 69(14): p. 5885-92.

133. Zhuang, Y., et al., Topotecan central nervous system penetration is altered by a tyrosine kinase inhibitor. Cancer Res, 2006. 66(23): p. 11305-13.

134. Polli, J.W., et al., The role of efflux and uptake transporters in [N-\{3-chloro-4[(3-fluorobenzyl)oxy]phenyl\}-6-[5-(\{[2-(methylsulfonyl)ethyl]amino \}methyl)-2furyl]-4-quinazolinamine (GW572016, lapatinib) disposition and drug interactions. Drug Metab Dispos, 2008. 36(4): p. 695-701.

135. Yasuda, K., et al., Drug transporters on arachnoid barrier cells contribute to the blood-cerebrospinal fluid barrier. Drug Metab Dispos, 2013. 41(4): p. 923-31.

136. Emmert, M.Y., et al., Higher frequencies of BCRP+ cardiac resident cells in ischaemic human myocardium. Eur Heart J, 2013. 34(36): p. 2830-8.

137. Liang, S.X. and W.D. Phillips, Migration of resident cardiac stem cells in myocardial infarction. Anat Rec (Hoboken), 2013. 296(2): p. 184-91. 
138. Hosomi, A., et al., Extra-renal elimination of uric acid via intestinal efflux transporter BCRP/ABCG2. PLoS One, 2012. 7(2): p. e30456.

139. Guo, Z., et al., Intestinal Microbiota Distinguish Gout Patients from Healthy Humans. Sci Rep, 2016. 6: p. 20602.

140. Bush, K.T., et al., The drug transporter OAT3 (SLC22A8) and endogenous metabolite communication via the gut-liver-kidney axis. J Biol Chem, 2017. 292(38): p. 15789-15803.

141. Wikoff, W.R., et al., Untargeted metabolomics identifies enterobiome metabolites and putative uremic toxins as substrates of organic anion transporter 1 (Oat1). $\mathrm{J}$ Proteome Res, 2011. 10(6): p. 2842-51.

142. Wu, W., K.T. Bush, and S.K. Nigam, Key Role for the Organic Anion Transporters, OAT1 and OAT3, in the in vivo Handling of Uremic Toxins and Solutes. Sci Rep, 2017. 7(1): p. 4939.

143. Cascorbi, I., P-glycoprotein: tissue distribution, substrates, and functional consequences of genetic variations. Handb Exp Pharmacol, 2011(201): p. 261-83.

144. Kort, A., et al., Brain Accumulation of Ponatinib and Its Active Metabolite, NDesmethyl Ponatinib, Is Limited by P-Glycoprotein (P-GP/ABCB1) and Breast Cancer Resistance Protein (BCRP/ABCG2). Mol Pharm, 2017. 14(10): p. 32583268.

145. Schuetz, E.G., K.N. Furuya, and J.D. Schuetz, Interindividual variation in expression of P-glycoprotein in normal human liver and secondary hepatic neoplasms. J Pharmacol Exp Ther, 1995. 275(2): p. 1011-8.

146. Ramesh, R., et al., Pro-inflammatory human Th17 cells selectively express Pglycoprotein and are refractory to glucocorticoids. J Exp Med, 2014. 211(1): p. 89-104.

147. Ueda, K., et al., The mdr1 gene, responsible for multidrug-resistance, codes for Pglycoprotein. Biochem Biophys Res Commun, 1986. 141(3): p. 956-62.

148. Thiebaut, F., et al., Cellular localization of the multidrug-resistance gene product P-glycoprotein in normal human tissues. Proc Natl Acad Sci U S A, 1987. 84(21): p. 7735-8.

149. Leschziner, G.D., et al., ABCB1 genotype and PGP expression, function and therapeutic drug response: a critical review and recommendations for future research. Pharmacogenomics J, 2007. 7(3): p. 154-79. 
150. Loscher, W. and H. Potschka, Role of drug efflux transporters in the brain for drug disposition and treatment of brain diseases. Prog Neurobiol, 2005. 76(1): p. 22-76.

151. Loscher, W., The pharmacokinetics of antiepileptic drugs in rats: consequences for maintaining effective drug levels during prolonged drug administration in rat models of epilepsy. Epilepsia, 2007. 48(7): p. 1245-58.

152. Liu, L. and X.D. Liu, Alterations in function and expression of ABC transporters at blood-brain barrier under diabetes and the clinical significances. Front Pharmacol, 2014. 5: p. 273.

153. Hartz, A.M. and B. Bauer, ABC transporters in the CNS - an inventory. Curr Pharm Biotechnol, 2011. 12(4): p. 656-73.

154. Bauer, B., et al., Seizure-induced up-regulation of P-glycoprotein at the bloodbrain barrier through glutamate and cyclooxygenase-2 signaling. Mol Pharmacol, 2008. 73(5): p. 1444-53.

155. Goralski, K.B., et al., Downregulation of mdr1a expression in the brain and liver during CNS inflammation alters the in vivo disposition of digoxin. $\mathrm{Br} \mathrm{J}$ Pharmacol, 2003. 139(1): p. 35-48.

156. Wessler, J.D., et al., The P-glycoprotein transport system and cardiovascular drugs. J Am Coll Cardiol, 2013. 61(25): p. 2495-502.

157. Srivalli, K.M.R. and P.K. Lakshmi, Overview of P-glycoprotein inhibitors: a rational outlook. Brazilian Journal of Pharmaceutical Sciences, 2012. 48: p. 353367.

158. Sawicki, E., et al., Clinical pharmacokinetics of an amorphous solid dispersion tablet of elacridar. Drug Deliv Transl Res, 2017. 7(1): p. 125-131.

159. Ueda, K., et al., Human P-glycoprotein transports cortisol, aldosterone, and dexamethasone, but not progesterone. J Biol Chem, 1992. 267(34): p. 24248-52.

160. Watchko, J.F., et al., P-glycoprotein and bilirubin disposition. J Perinatol, 2001. 21 Suppl 1: p. S43-7; discussion S59-62.

161. van Helvoort, A., et al., MDR1 P-glycoprotein is a lipid translocase of broad specificity, while MDR3 P-glycoprotein specifically translocates phosphatidylcholine. Cell, 1996. 87(3): p. 507-17.

162. Nones, K., et al., Multidrug resistance gene deficient (mdr1a-/-) mice have an altered caecal microbiota that precedes the onset of intestinal inflammation. $\mathrm{J}$ Appl Microbiol, 2009. 107(2): p. 557-66. 
163. Englund, G., et al., Efflux transporters in ulcerative colitis: decreased expression of BCRP (ABCG2) and Pgp (ABCB1). Inflamm Bowel Dis, 2007. 13(3): p. 2917.

164. Hartz, A.M., et al., Abeta40 Reduces P-Glycoprotein at the Blood-Brain Barrier through the Ubiquitin-Proteasome Pathway. J Neurosci, 2016. 36(6): p. 1930-41.

165. Chen, Y., et al., P-glycoprotein and breast cancer resistance protein influence brain distribution of dasatinib. J Pharmacol Exp Ther, 2009. 330(3): p. 956-63.

166. Hu, S., et al., Interaction of the multikinase inhibitors sorafenib and sunitinib with solute carriers and ATP-binding cassette transporters. Clin Cancer Res, 2009. 15(19): p. 6062-9.

167. Agarwal, S., et al., Breast cancer resistance protein and P-glycoprotein in brain cancer: two gatekeepers team up. Curr Pharm Des, 2011. 17(26): p. 2793-802.

168. Kannan, P., et al., Factors that limit positron emission tomography imaging of pglycoprotein density at the blood-brain barrier. Mol Pharm, 2013. 10(6): p. 22229 .

169. Uchida, Y., et al., Quantitative targeted absolute proteomics of human blood-brain barrier transporters and receptors. J Neurochem, 2011. 117(2): p. 333-45.

170. Braun, C., et al., Quantification of Transporter and Receptor Proteins in Dog Brain Capillaries and Choroid Plexus: Relevance for the Distribution in Brain and CSF of Selected BCRP and P-gp Substrates. Mol Pharm, 2017. 14(10): p. 34363447.

171. Uchida, Y., et al., Quantitative targeted absolute proteomics of rat bloodcerebrospinal fluid barrier transporters: comparison with a human specimen. $\mathrm{J}$ Neurochem, 2015. 134(6): p. 1104-15.

172. Redzic, Z., Molecular biology of the blood-brain and the blood-cerebrospinal fluid barriers: similarities and differences. Fluids Barriers CNS, 2011. 8(1): p. 3.

173. Dallas, S., D.S. Miller, and R. Bendayan, Multidrug resistance-associated proteins: expression and function in the central nervous system. Pharmacol Rev, 2006. 58(2): p. 140-61.

174. Rao, V.V., et al., Choroid plexus epithelial expression of MDR1 P glycoprotein and multidrug resistance-associated protein contribute to the blood-cerebrospinalfluid drug-permeability barrier. Proc Natl Acad Sci U S A, 1999. 96(7): p. 3900-5.

175. Shugarts, S. and L.Z. Benet, The role of transporters in the pharmacokinetics of orally administered drugs. Pharm Res, 2009. 26(9): p. 2039-54. 
176. Padowski, J.M. and G.M. Pollack, Pharmacokinetic and pharmacodynamic implications of P-glycoprotein modulation. Methods Mol Biol, 2010. 596: p. 35984.

177. Roberts, J.K., et al., Population Pharmacokinetics of Oral Topotecan in Infants and Very Young Children with Brain Tumors Demonstrates a Role of ABCG2 rs4148157 on the Absorption Rate Constant. Drug Metab Dispos, 2016. 44(7): p. 1116-22.

178. Mukherjee, K., et al., Whole blood transcriptomics and urinary metabolomics to define adaptive biochemical pathways of high-intensity exercise in 50-60 year old masters athletes. PLoS One, 2014. 9(3): p. e92031.

179. Roberts, L.D., et al., Targeted metabolomics. Curr Protoc Mol Biol, 2012. Chapter 30: p. Unit 302 1-24.

180. Kaddurah-Daouk, R., R.M. Weinshilboum, and N. Pharmacometabolomics Research, Pharmacometabolomics: implications for clinical pharmacology and systems pharmacology. Clin Pharmacol Ther, 2014. 95(2): p. 154-67.

181. Raamsdonk, L.M., et al., A functional genomics strategy that uses metabolome data to reveal the phenotype of silent mutations. Nat Biotechnol, 2001. 19(1): p. 45-50.

182. Yu, B., et al., Associations Between the Serum Metabolome and All-Cause Mortality Among African Americans in the Atherosclerosis Risk in Communities (ARIC) Study. Am J Epidemiol, 2016. 183(7): p. 650-6.

183. Cassol, E., et al., Cerebrospinal fluid metabolomics reveals altered waste clearance and accelerated aging in HIV patients with neurocognitive impairment. AIDS, 2014. 28(11): p. 1579-91.

184. Ament, Z., M. Masoodi, and J.L. Griffin, Applications of metabolomics for understanding the action of peroxisome proliferator-activated receptors (PPARs) in diabetes, obesity and cancer. Genome Med, 2012. 4(4): p. 32.

185. Beger, R.D., et al., Metabolomics enables precision medicine: "A White Paper, Community Perspective". Metabolomics, 2016. 12(10): p. 149.

186. Evans, A.M., et al., Integrated, nontargeted ultrahigh performance liquid chromatography/electrospray ionization tandem mass spectrometry platform for the identification and relative quantification of the small-molecule complement of biological systems. Anal Chem, 2009. 81(16): p. 6656-67.

187. Wishart, D.S., et al., HMDB 4.0: the human metabolome database for 2018. Nucleic Acids Res, 2018. 46(D1): p. D608-D617. 
188. van de Wetering, K., et al., Targeted metabolomics identifies glucuronides of dietary phytoestrogens as a major class of MRP3 substrates in vivo.

Gastroenterology, 2009. 137(5): p. 1725-35.

189. Xia, J., et al., MetaboAnalyst 3.0--making metabolomics more meaningful. Nucleic Acids Res, 2015. 43(W1): p. W251-7.

190. Tautenhahn, R., et al., metaXCMS: second-order analysis of untargeted metabolomics data. Anal Chem, 2011. 83(3): p. 696-700.

191. Kastenmuller, G., et al., metaP-server: a web-based metabolomics data analysis tool. J Biomed Biotechnol, 2011. 2011.

192. Cambiaghi, A., M. Ferrario, and M. Masseroli, Analysis of metabolomic data: tools, current strategies and future challenges for omics data integration. Brief Bioinform, 2017. 18(3): p. 498-510.

193. Brink-Jensen, K., et al., Integrative analysis of metabolomics and transcriptomics data: a unified model framework to identify underlying system pathways. PLoS One, 2013. 8(9): p. e72116.

194. Cavill, R., et al., Transcriptomic and metabolomic data integration. Brief Bioinform, 2016. 17(5): p. 891-901.

195. Lu, Y., et al., Comparative transcriptomic and metabolomic analysis of fenofibrate and fish oil treatments in mice. Physiol Genomics, 2011. 43(23): p. 1307-18.

196. Kelly, R.S., et al., Integration of metabolomic and transcriptomic networks in pregnant women reveals biological pathways and predictive signatures associated with preeclampsia. Metabolomics, 2017. 13(1).

197. Watkins, S.M., et al., Lipid metabolome-wide effects of the PPARgamma agonist rosiglitazone. J Lipid Res, 2002. 43(11): p. 1809-17.

198. Yee, S.W., et al., Metabolomic and Genome-wide Association Studies Reveal Potential Endogenous Biomarkers for OATP1B1. Clin Pharmacol Ther, 2016. 100(5): p. 524-536.

199. Shin, S.Y., et al., An atlas of genetic influences on human blood metabolites. Nat Genet, 2014. 46(6): p. 543-550.

200. Suhre, K., et al., Human metabolic individuality in biomedical and pharmaceutical research. Nature, 2011. 477(7362): p. 54-60. 
201. Sampath, J., et al., Role of MRP4 and MRP5 in biology and chemotherapy. AAPS PharmSci, 2002. 4(3): p. E14.

202. Lancaster, C.S., et al., Cisplatin-induced downregulation of OCTN2 affects carnitine wasting. Clin Cancer Res, 2010. 16(19): p. 4789-99.

203. Grundemann, D., et al., Transport of monoamine transmitters by the organic cation transporter type 2, OCT2. J Biol Chem, 1998. 273(47): p. 30915-20.

204. Matsson, P., et al., Identification of novel specific and general inhibitors of the three major human ATP-binding cassette transporters P-gp, BCRP and MRP2 among registered drugs. Pharm Res, 2009. 26(8): p. 1816-31.

205. Huls, M., F.G. Russel, and R. Masereeuw, The role of ATP binding cassette transporters in tissue defense and organ regeneration. J Pharmacol Exp Ther, 2009. 328(1): p. 3-9.

206. Ashraf, T., et al., Drug transporters at brain barriers: expression and regulation by neurological disorders. Adv Exp Med Biol, 2012. 763: p. 20-69.

207. Shen, S., et al., ABCG2 reduces ROS-mediated toxicity and inflammation: a potential role in Alzheimer's disease. J Neurochem, 2010. 114(6): p. 1590-604.

208. Choudhuri, S. and C.D. Klaassen, Structure, function, expression, genomic organization, and single nucleotide polymorphisms of human ABCB1 (MDR1), ABCC (MRP), and ABCG2 (BCRP) efflux transporters. Int J Toxicol, 2006. 25(4): p. 231-59.

209. Zhang, G., et al., Integration of metabolomics and transcriptomics revealed a fatty acid network exerting growth inhibitory effects in human pancreatic cancer. Clin Cancer Res, 2013. 19(18): p. 4983-93.

210. Bjerrum, J.T., et al., Integration of transcriptomics and metabonomics: improving diagnostics, biomarker identification and phenotyping in ulcerative colitis. Metabolomics, 2014. 10(2): p. 280-290.

211. Song, I.S., et al., Pharmacogenetics meets metabolomics: discovery of tryptophan as a new endogenous OCT2 substrate related to metformin disposition. PLoS One, 2012. 7(5): p. e36637.

212. Velenosi, T.J., et al., Untargeted plasma and tissue metabolomics in rats with chronic kidney disease given AST-120. Sci Rep, 2016. 6: p. 22526.

213. Martinez, D., et al., Endogenous Metabolites-Mediated Communication Between OAT1/OAT3 and OATP1B1 May Explain the Association Between SLCO1B1 SNPs and Methotrexate Toxicity. Clin Pharmacol Ther, 2017. 
214. Lin, Y.S., et al., Prediction of Intravenous Busulfan Clearance by Endogenous Plasma Biomarkers Using Global Pharmacometabolomics. Metabolomics, 2016. 12(10).

215. Merino, G., et al., Sex-dependent expression and activity of the ATP-binding cassette transporter breast cancer resistance protein (BCRP/ABCG2) in liver. Mol Pharmacol, 2005. 67(5): p. 1765-71.

216. Nording, M.L., et al., Individual variation in lipidomic profiles of healthy subjects in response to omega-3 Fatty acids. PLoS One, 2013. 8(10): p. e76575.

217. Clark, A.M., et al., Open Source Bayesian Models. 1. Application to ADME/Tox and Drug Discovery Datasets. J Chem Inf Model, 2015. 55(6): p. 1231-45.

218. Clark, A.M. and S. Ekins, Open Source Bayesian Models. 2. Mining a "Big Dataset" To Create and Validate Models with ChEMBL. J Chem Inf Model, 2015. 55(6): p. 1246-60.

219. Kazos, E.A., et al., Simultaneous determination of chlorothalonil and its metabolite 4-hydroxychlorothalonil in greenhouse air: dissipation process of chlorothalonil. Chemosphere, 2008. 72(10): p. 1413-9.

220. Alvarez, A.I., et al., Bioavailability of the glucuronide and sulfate conjugates of genistein and daidzein in breast cancer resistance protein 1 knockout mice. Drug Metab Dispos, 2011. 39(11): p. 2008-12.

221. Takenaka, K., et al., Substrate overlap between Mrp4 and Abcg2/Bcrp affects purine analogue drug cytotoxicity and tissue distribution. Cancer Res, 2007. 67(14): p. 6965-72.

222. Patterson, A.D., et al., Human urinary metabolomic profile of PPARalpha induced fatty acid beta-oxidation. J Proteome Res, 2009. 8(9): p. 4293-300.

223. Bjorndal, B., et al., Fish oil and the pan-PPAR agonist tetradecylthioacetic acid affect the amino acid and carnitine metabolism in rats. PLoS One, 2013. 8(6): p. e66926.

224. Zhen, Y., et al., Metabolomic and genetic analysis of biomarkers for peroxisome proliferator-activated receptor alpha expression and activation. Mol Endocrinol, 2007. 21(9): p. 2136-51.

225. Yang, Z., et al., Breast cancer resistance protein (ABCG2) determines distribution of genistein phase II metabolites: reevaluation of the roles of ABCG2 in the disposition of genistein. Drug Metab Dispos, 2012. 40(10): p. 1883-93. 
226. Kim, S., et al., Genistein enhances expression of genes involved in fatty acid catabolism through activation of PPARalpha. Mol Cell Endocrinol, 2004. 220(12): p. 51-8.

227. Ronis, M.J., et al., Dietary soy protein isolate attenuates metabolic syndrome in rats via effects on PPAR, LXR, and SREBP signaling. J Nutr, 2009. 139(8): p. $1431-8$.

228. Shin, E.S., et al., Genistein downregulates SREBP-1 regulated gene expression by inhibiting site-1 protease expression in HepG2 cells. J Nutr, 2007. 137(5): p. $1127-31$.

229. Wang, L., et al., Natural product agonists of peroxisome proliferator-activated receptor gamma (PPARgamma): a review. Biochem Pharmacol, 2014. 92(1): p. 73-89.

230. Cho, K.W., et al., Daidzein and the daidzein metabolite, equol, enhance adipocyte differentiation and PPARgamma transcriptional activity. J Nutr Biochem, 2010. 21(9): p. 841-7.

231. Sakamoto, Y., et al., The Dietary Isoflavone Daidzein Reduces Expression of ProInflammatory Genes through PPARalpha/gamma and JNK Pathways in Adipocyte and Macrophage Co-Cultures. PLoS One, 2016. 11(2): p. e0149676.

232. Dai, D., et al., Time-resolved metabolomics analysis of individual differences during the early stage of lipopolysaccharide-treated rats. Sci Rep, 2016. 6: p. 34136.

233. Manco, M., L. Putignani, and G.F. Bottazzo, Gut microbiota, lipopolysaccharides, and innate immunity in the pathogenesis of obesity and cardiovascular risk. Endocr Rev, 2010. 31(6): p. 817-44.

234. Wikoff, W.R., et al., Metabolomics analysis reveals large effects of gut microflora on mammalian blood metabolites. Proc Natl Acad Sci U S A, 2009. 106(10): p. 3698-703.

235. Bone, E., A. Tamm, and M. Hill, The production of urinary phenols by gut bacteria and their possible role in the causation of large bowel cancer. Am J Clin Nutr, 1976. 29(12): p. 1448-54.

236. Schuetz, E.G., et al., The glucocorticoid receptor is essential for induction of cytochrome P-4502B by steroids but not for drug or steroid induction of CYP3A or P-450 reductase in mouse liver. Drug Metab Dispos, 2000. 28(3): p. 268-78. 
237. Xiao, G., et al., Cerebrospinal fluid can be used as a surrogate to assess brain exposures of breast cancer resistance protein and P-glycoprotein substrates. Drug Metab Dispos, 2012. 40(4): p. 779-87.

238. Kutzing, M.K. and B.L. Firestein, Altered uric acid levels and disease states. J Pharmacol Exp Ther, 2008. 324(1): p. 1-7.

239. Crotty, G.F., A. Ascherio, and M.A. Schwarzschild, Targeting urate to reduce oxidative stress in Parkinson disease. Exp Neurol, 2017. 298(Pt B): p. 210-224.

240. Glantzounis, G.K., et al., Uric acid and oxidative stress. Curr Pharm Des, 2005. 11(32): p. 4145-51.

241. Choi, Y.J., et al., Uric acid induces fat accumulation via generation of endoplasmic reticulum stress and SREBP-1c activation in hepatocytes. Lab Invest, 2014. 94(10): p. 1114-25.

242. Schoneich, C., Methionine oxidation by reactive oxygen species: reaction mechanisms and relevance to Alzheimer's disease. Biochim Biophys Acta, 2005. 1703(2): p. 111-9.

243. Cantor, J.R., et al., Physiologic Medium Rewires Cellular Metabolism and Reveals Uric Acid as an Endogenous Inhibitor of UMP Synthase. Cell, 2017. 169(2): p. 258-272 e17.

244. Matta-Camacho, E., et al., Structure of REV-ERBbeta ligand-binding domain bound to a porphyrin antagonist. J Biol Chem, 2014. 289(29): p. 20054-66.

245. Hamdan, A.M., et al., Intestinal expression of mouse Abcg2/breast cancer resistance protein (BCRP) gene is under control of circadian clock-activating transcription factor-4 pathway. J Biol Chem, 2012. 287(21): p. 17224-31.

246. Zhou, S., et al., Increased expression of the Abcg2 transporter during erythroid maturation plays a role in decreasing cellular protoporphyrin IX levels. Blood, 2005. 105(6): p. 2571-6.

247. Sleigh J, H.M., Voss L, Denny B, Ketamine - More mechanisms of action than just NMDA blockade. Trends in anesthesia and critical care 2014. 4(2-3): p. 7681.

248. Niesters, M., C. Martini, and A. Dahan, Ketamine for chronic pain: risks and benefits. Br J Clin Pharmacol, 2014. 77(2): p. 357-67.

249. Barrett, T.W. and D.L. Schriger, Move Over Morphine: Is Ketamine an Effective and Safe Alternative for Treating Acute Pain?: Answers to the September 2015 Journal Club. Ann Emerg Med, 2016. 67(2): p. 289-94. 
250. Machado-Vieira, R., et al., Ketamine and the next generation of antidepressants with a rapid onset of action. Pharmacol Ther, 2009. 123(2): p. 143-50.

251. Shahani, R., et al., Ketamine-associated ulcerative cystitis: a new clinical entity. Urology, 2007. 69(5): p. 810-2.

252. Chu, P.S., et al., The destruction of the lower urinary tract by ketamine abuse: a new syndrome? BJU Int, 2008. 102(11): p. 1616-22.

253. Mion, G. and T. Villevieille, Ketamine pharmacology: an update (pharmacodynamics and molecular aspects, recent findings). CNS Neurosci Ther, 2013. 19(6): p. 370-80.

254. Sherwin, C.M., et al., Development of an optimal sampling schedule for children receiving ketamine for short-term procedural sedation and analgesia. Paediatr Anaesth, 2015. 25(2): p. 211-6.

255. Fanta, S., et al., Population pharmacokinetics of S-ketamine and norketamine in healthy volunteers after intravenous and oral dosing. Eur J Clin Pharmacol, 2015. 71(4): p. 441-7.

256. Hijazi, Y. and R. Boulieu, Contribution of CYP3A4, CYP2B6, and CYP2C9 isoforms to N-demethylation of ketamine in human liver microsomes. Drug Metab Dispos, 2002. 30(7): p. 853-8.

257. Grant, I.S., W.S. Nimmo, and J.A. Clements, Pharmacokinetics and analgesic effects of i.m. and oral ketamine. Br J Anaesth, 1981. 53(8): p. 805-10.

258. Varma, M.V., et al., P-glycoprotein inhibitors and their screening: a perspective from bioavailability enhancement. Pharmacol Res, 2003. 48(4): p. 347-59.

259. Kusuhara, H., et al., Pharmacokinetic interaction study of sulphasalazine in healthy subjects and the impact of curcumin as an in vivo inhibitor of BCRP. Br J Pharmacol, 2012. 166(6): p. 1793-803.

260. Lilius, T.O., et al., Ketamine coadministration attenuates morphine tolerance and leads to increased brain concentrations of both drugs in the rat. Br J Pharmacol, 2015. 172(11): p. 2799-813.

261. Sato, Y., et al., The differential effect of cyclosporine on hypnotic response and pain reaction in mice. Anesth Analg, 2007. 105(5): p. 1489-93, table of contents.

262. Poller, B., et al., Double-transduced MDCKII cells to study human P-glycoprotein $(\mathrm{ABCB} 1)$ and breast cancer resistance protein (ABCG2) interplay in drug transport across the blood-brain barrier. Mol Pharm, 2011. 8(2): p. 571-82. 
263. Moore, K.A., et al., Urine concentrations of ketamine and norketamine following illegal consumption. J Anal Toxicol, 2001. 25(7): p. 583-8.

264. Bolze, S. and R. Boulieu, HPLC determination of ketamine, norketamine, and dehydronorketamine in plasma with a high-purity reversed-phase sorbent. Clin Chem, 1998. 44(3): p. 560-4.

265. Jaki, T. and M.J. Wolfsegger, Estimation of pharmacokinetic parameters with the R package PK. Pharmaceutical Statistics, 2011. 10(3): p. 284-288.

266. Kuhn, E. and M. Lavielle, Maximum likelihood estimation in nonlinear mixed effects models. Computational Statistics \& Data Analysis, 2005. 49(4): p. 10201038.

267. Sato, Y., et al., Chronopharmacological studies of ketamine in normal and NMDA epsilon1 receptor knockout mice. Br J Anaesth, 2004. 92(6): p. 859-64.

268. Lagas, J.S., et al., Brain accumulation of dasatinib is restricted by P-glycoprotein $(\mathrm{ABCB} 1)$ and breast cancer resistance protein $(\mathrm{ABCG} 2)$ and can be enhanced by elacridar treatment. Clin Cancer Res, 2009. 15(7): p. 2344-51.

269. Sane, R., S. Agarwal, and W.F. Elmquist, Brain distribution and bioavailability of elacridar after different routes of administration in the mouse. Drug Metab Dispos, 2012. 40(8): p. 1612-9.

270. Hendrikx, J.J., et al., Oral co-administration of elacridar and ritonavir enhances plasma levels of oral paclitaxel and docetaxel without affecting relative brain accumulation. Br J Cancer, 2014. 110(11): p. 2669-76.

271. Durmus, S., et al., Oral availability and brain penetration of the B-RAFV600E inhibitor vemurafenib can be enhanced by the P-GLYCOprotein (ABCB1) and breast cancer resistance protein (ABCG2) inhibitor elacridar. Mol Pharm, 2012. 9(11): p. 3236-45.

272. Tournier, N., et al., Interaction of drugs of abuse and maintenance treatments with human P-glycoprotein (ABCB1) and breast cancer resistance protein (ABCG2). Int J Neuropsychopharmacol, 2010. 13(7): p. 905-15.

273. Winters, W.D., et al., Seasonal and sex influences on ketamine-induced analgesia and catalepsy in the rat; a possible role for melatonin. Neuropharmacology, 1986. 25(10): p. 1095-101.

274. Zanos, P., et al., NMDAR inhibition-independent antidepressant actions of ketamine metabolites. Nature, 2016. 533(7604): p. 481-6. 
275. Can, A., et al., Effects of Ketamine and Ketamine Metabolites on Evoked Striatal Dopamine Release, Dopamine Receptors, and Monoamine Transporters. J Pharmacol Exp Ther, 2016. 359(1): p. 159-70.

276. Cohen, M.L., et al., Distribution in the brain and metabolism of ketamine in the rat after intravenous administration. Anesthesiology, 1973. 39(4): p. 370-6.

277. Bexten, M., et al., Expression of drug transporters and drug metabolizing enzymes in the bladder urothelium in man and affinity of the bladder spasmolytic trospium chloride to transporters likely involved in its pharmacokinetics. Mol Pharm, 2015. 12(1): p. 171-8.

278. Weiss, J., et al., Inhibition of P-glycoprotein by newer antidepressants. J Pharmacol Exp Ther, 2003. 305(1): p. 197-204.

279. Hilgendorf, C., et al., Expression of thirty-six drug transporter genes in human intestine, liver, kidney, and organotypic cell lines. Drug Metab Dispos, 2007. 35(8): p. 1333-40.

280. Alcorn, J. and P.J. McNamara, Acyclovir, ganciclovir, and zidovudine transfer into rat milk. Antimicrob Agents Chemother, 2002. 46(6): p. 1831-6.

281. Jonker, J.W., et al., Role of breast cancer resistance protein in the bioavailability and fetal penetration of topotecan. J Natl Cancer Inst, 2000. 92(20): p. 1651-6.

282. Dahan, A. and G.L. Amidon, MRP2 mediated drug-drug interaction: indomethacin increases sulfasalazine absorption in the small intestine, potentially decreasing its colonic targeting. Int J Pharm, 2010. 386(1-2): p. 216-20.

283. Kunze, A., et al., Prediction of organic anion-transporting polypeptide 1B1- and 1B3-mediated hepatic uptake of statins based on transporter protein expression and activity data. Drug Metab Dispos, 2014. 42(9): p. 1514-21.

284. Ellis, L.C., G.M. Hawksworth, and R.J. Weaver, ATP-dependent transport of statins by human and rat MRP2/Mrp2. Toxicol Appl Pharmacol, 2013. 269(2): p. $187-94$.

285. Li, J., Y. Wang, and I.J. Hidalgo, Kinetic analysis of human and canine Pglycoprotein-mediated drug transport in MDR1-MDCK cell model: approaches to reduce false-negative substrate classification. J Pharm Sci, 2013. 102(9): p. 343646.

286. Pan, G., et al., Organic cation uptake is enhanced in bcrp1-transfected MDCKII cells. Mol Pharm, 2010. 7(1): p. 138-45. 
287. Kraatz, M., et al., Effects of Chlorophyll-Derived Efflux Pump Inhibitor Pheophorbide a and Pyropheophorbide a on Growth and Macrolide Antibiotic Resistance of Indicator and Anaerobic Swine Manure Bacteria. International Journal of Antibiotics, 2014. 2014: p. 14.

288. Robey, R.W., et al., Pheophorbide a is a specific probe for ABCG2 function and inhibition. Cancer Res, 2004. 64(4): p. 1242-6.

289. Zhang, W., et al., Silencing the breast cancer resistance protein expression and function in caco-2 cells using lentiviral vector-based short hairpin RNA. Drug Metab Dispos, 2009. 37(4): p. 737-44.

290. Eichwurzel, I., H. Stiel, and B. Roder, Photophysical studies of the pheophorbide a dimer. J Photochem Photobiol B, 2000. 54(2-3): p. 194-200.

291. Ruiz-Gonzalez, R., et al., Photodynamic Synergistic Effect of Pheophorbide a and Doxorubicin in Combined Treatment against Tumoral Cells. Cancers (Basel), 2017. 9(2).

292. Ardeshirpour, Y., et al., Using in-vivo fluorescence imaging in personalized cancer diagnostics and therapy, an image and treat paradigm. Technol Cancer Res Treat, 2011. 10(6): p. 549-60.

293. Pavek, P., et al., Human breast cancer resistance protein: interactions with steroid drugs, hormones, the dietary carcinogen 2-amino-1-methyl-6-phenylimidazo(4,5b)pyridine, and transport of cimetidine. J Pharmacol Exp Ther, 2005. 312(1): p. $144-52$.

294. Almela, L., J.A. Fernandez-Lopez, and M.J. Roca, High-performance liquid chromatographic screening of chlorophyll derivatives produced during fruit storage. J Chromatogr A, 2000. 870(1-2): p. 483-9.

295. Weiss, J., et al., Modulation of human BCRP (ABCG2) activity by anti-HIV drugs. J Antimicrob Chemother, 2007. 59(2): p. 238-45.

296. Mahringer, A., J. Delzer, and G. Fricker, A fluorescence-based in vitro assay for drug interactions with breast cancer resistance protein (BCRP, ABCG2). Eur J Pharm Biopharm, 2009. 72(3): p. 605-13.

297. Bhaumik, S., J. DePuy, and J. Klimash, Strategies to minimize background autofluorescence in live mice during noninvasive fluorescence optical imaging. Lab Anim (NY), 2007. 36(8): p. 40-3.

298. Leblond, F., et al., Pre-clinical whole-body fluorescence imaging: Review of instruments, methods and applications. J Photochem Photobiol B, 2010. 98(1): p. 77-94. 
299. Kuppens, I.E., et al., A phase I, randomized, open-label, parallel-cohort, dosefinding study of elacridar (GF120918) and oral topotecan in cancer patients. Clin Cancer Res, 2007. 13(11): p. 3276-85.

300. Wang, Q., M. Zheng, and T. Leil, Investigating Transporter-Mediated Drug-Drug Interactions Using a Physiologically Based Pharmacokinetic Model of Rosuvastatin. CPT Pharmacometrics Syst Pharmacol, 2017. 6(4): p. 228-238.

301. Bakhsheshian, J., et al., Overlapping substrate and inhibitor specificity of human and murine ABCG2. Drug Metab Dispos, 2013. 41(10): p. 1805-12.

302. Hortensteiner, S., et al., The key step in chlorophyll breakdown in higher plants. Cleavage of pheophorbide a macrocycle by a monooxygenase. J Biol Chem, 1998. 273(25): p. 15335-9.

303. Jitsukawa, K., R. Suizu, and A. Hidano, Chlorella photosensitization. New phytophotodermatosis. Int J Dermatol, 1984. 23(4): p. 263-8.

304. Ahn, M.Y., et al., Pheophorbide a-mediated photodynamic therapy induces apoptotic cell death in murine oral squamous cell carcinoma in vitro and in vivo. Oncol Rep, 2012. 27(6): p. 1772-8.

305. Strumberg, D., et al., Safety, pharmacokinetics, and preliminary antitumor activity of sorafenib: a review of four phase I trials in patients with advanced refractory solid tumors. Oncologist, 2007. 12(4): p. 426-37.

306. McConnell, E.L., A.W. Basit, and S. Murdan, Measurements of rat and mouse gastrointestinal $\mathrm{pH}$, fluid and lymphoid tissue, and implications for in-vivo experiments. J Pharm Pharmacol, 2008. 60(1): p. 63-70.

307. Villarroel, M.C., et al., Plasma protein binding of sorafenib, a multi kinase inhibitor: in vitro and in cancer patients. Invest New Drugs, 2012. 30(6): p. 2096102.

308. Kamath, A.V., et al., Preclinical pharmacokinetics and in vitro metabolism of dasatinib (BMS-354825): a potent oral multi-targeted kinase inhibitor against SRC and BCR-ABL. Cancer Chemother Pharmacol, 2008. 61(3): p. 365-76.

309. Suzuki, K., et al., Co-administration of proton pump inhibitors delays elimination of plasma methotrexate in high-dose methotrexate therapy. Br J Clin Pharmacol, 2009. 67(1): p. 44-9.

310. Ahmed-Belkacem, A., et al., Flavonoid structure-activity studies identify 6prenylchrysin and tectochrysin as potent and specific inhibitors of breast cancer resistance protein ABCG2. Cancer Res, 2005. 65(11): p. 4852-60. 
311. Hudachek, S.F. and D.L. Gustafson, Incorporation of ABCB1-mediated transport into a physiologically-based pharmacokinetic model of docetaxel in mice. J Pharmacokinet Pharmacodyn, 2013. 40(4): p. 437-49.

312. Zhongfa, L., et al., Enhancement of curcumin oral absorption and pharmacokinetics of curcuminoids and curcumin metabolites in mice. Cancer Chemother Pharmacol, 2012. 69(3): p. 679-89.

313. Maier, L., et al., Extensive impact of non-antibiotic drugs on human gut bacteria. Nature, 2018. 555(7698): p. 623-628.

314. Shen, D.D., A.A. Artru, and K.K. Adkison, Principles and applicability of CSF sampling for the assessment of CNS drug delivery and pharmacodynamics. Adv Drug Deliv Rev, 2004. 56(12): p. 1825-57.

315. Meeks, J.P., H.A. Arnson, and T.E. Holy, Representation and transformation of sensory information in the mouse accessory olfactory system. Nat Neurosci, 2010. 13(6): p. 723-30.

316. Imai, Y., et al., Breast cancer resistance protein exports sulfated estrogens but not free estrogens. Mol Pharmacol, 2003. 64(3): p. 610-8.

317. Feng, Q., W.D. Chen, and Y.D. Wang, Gut Microbiota: An Integral Moderator in Health and Disease. Front Microbiol, 2018. 9: p. 151.

318. Yang, Y., J. Tian, and B. Yang, Targeting gut microbiome: A novel and potential therapy for autism. Life Sci, 2018. 194: p. 111-119.

319. Ochoa-Reparaz, J., T.O. Kirby, and L.H. Kasper, The Gut Microbiome and Multiple Sclerosis. Cold Spring Harb Perspect Med, 2018.

320. de Lange, E.C.M., et al., Novel CNS drug discovery and development approach: model-based integration to predict neuro-pharmacokinetics and pharmacodynamics. Expert Opin Drug Discov, 2017. 12(12): p. 1207-1218.

321. van den Brink, W.J., et al., Multivariate pharmacokinetic/pharmacodynamic (PKPD) analysis with metabolomics shows multiple effects of remoxipride in rats. Eur J Pharm Sci, 2017. 109: p. 431-440.

322. Mason, K., et al., Ketamine-associated lower urinary tract destruction: a new radiological challenge. Clin Radiol, 2010. 65(10): p. 795-800.

323. Pal, R., et al., Ketamine is associated with lower urinary tract signs and symptoms. Drug Alcohol Depend, 2013. 132(1-2): p. 189-94. 


\section{VITA}

Samit Ganguly was born in 1982 in West Bengal, India. He spent his childhood and finished high school in the industrial town Durgapur, near Calcutta, India. Samit obtained his Bachelor in Pharmaceutical Technology degree from Jadavpur University in Kolkata in 2005, and a Masters in Pharmaceutical Sciences from Birla Institute of Technology and Science (BITS), Pilani in 2007. During this time, Samit did internships at different pharmaceutical companies such as Albert David and thereafter at Wockhardt Research Center in the Department of Pharmacokinetics and Safety Pharmacology, where he performed research on assessing neuropathic pain precipitation risk in rodents for long-term linezolid therapy. After finishing his Masters degree, Samit worked for 5 years as Research Associate and Assistant Research Scientist II in the Division of Drug Metabolism and Pharmacokinetics at AstraZeneca India Pvt. Ltd, Bangalore, India. During his time at AstraZeneca, Samit was extensively involved in the pharmacokinetic characterization and decision-making for the progress of tuberculosis and malaria drug discovery molecules at hit identification, lead identification and optimization stages.

In 2012, Samit joined the PhD program in Biomedical Sciences at University of Tennessee Health Science Center (UTHSC), and after a year of laboratory rotations, started pursuing his research work under the mentorship of Dr. Erin G. Schuetz, as part of the interdisciplinary PhD program of UTHSC and St Jude Children's Research Hospital. During his $\mathrm{PhD}$ training, Samit received numerous travel awards and presented his research work at several national and international scientific conferences including the Gordon Research Conference, and meetings of International Society for the Study of Xenobiotics and American Association of Pharmaceutical Sciences. In addition, Samit was actively involved in a leadership position at Life Science Tennessee Mid-South Academic Alliance, and helped organize the inaugural Memphis Scipreneur Challenge. After completion of his $\mathrm{PhD}$, Samit plans to pursue further postdoctoral training in the field of pediatric clinical pharmacology and pharmacometrics. 